\title{
Pentagon-Containing $\pi$-Expanded Systems: Synthesis and Photophysical Properties
}

Xin Deng, Xinqun Liu, Leping Wei, Tongtong Ye, Xiaohui Yu, Chunfang Zhang* and Jinchong Xiao*

College of Chemistry and Environmental Science, Key Laboratory of Chemical Biology of Hebei Province, Key Laboratory of Medicinal Chemistry and Molecular Diagnosis, Ministry of Education, Hebei University, Baoding 071002, P. R. China.

\section{Content}

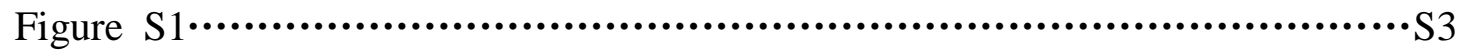

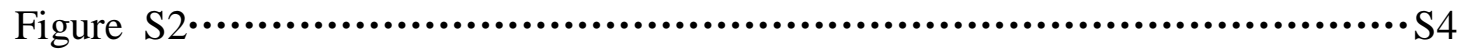

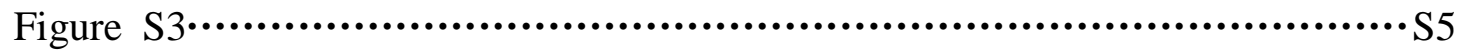

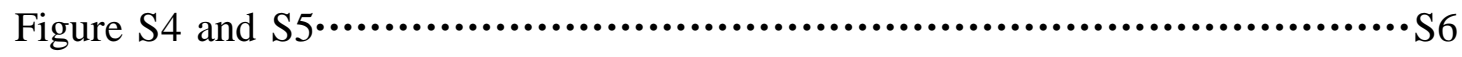

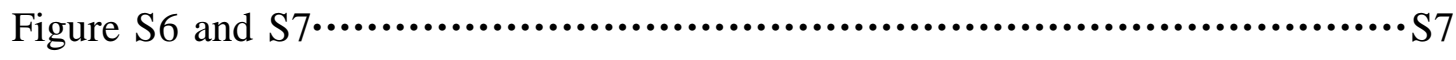

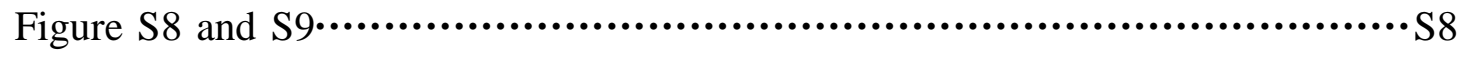

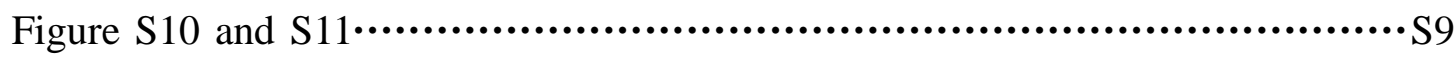

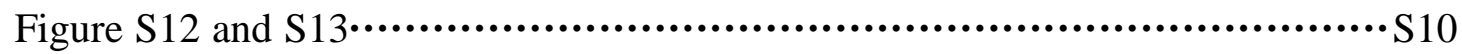

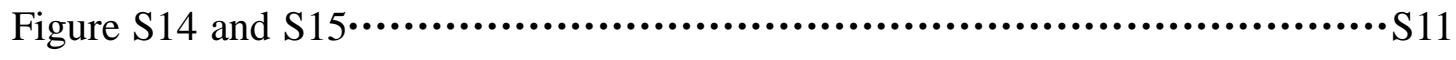

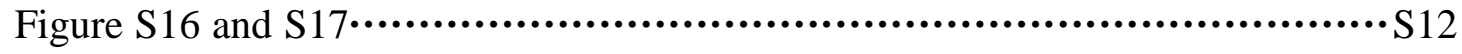

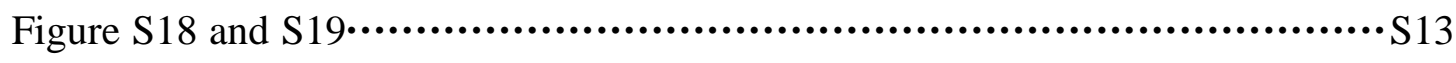

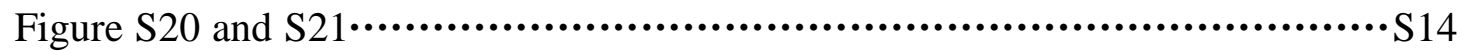

Figure $S 22$ and S23

Figure S24 and S25

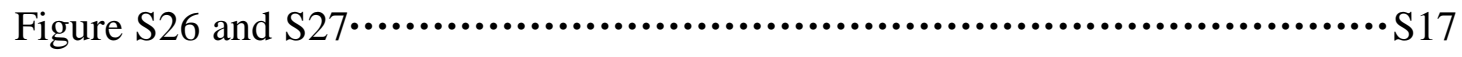

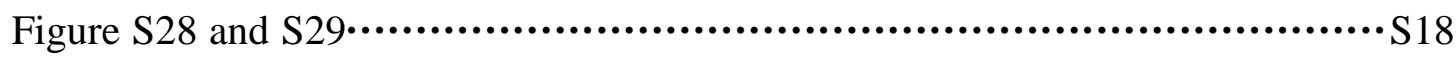

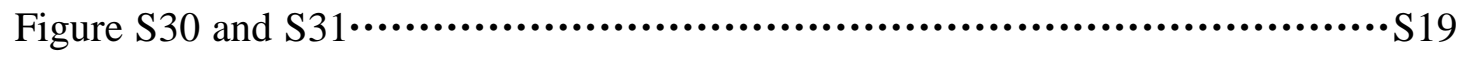

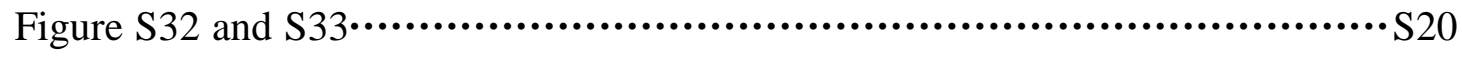

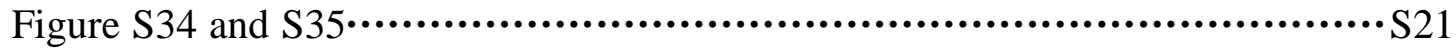

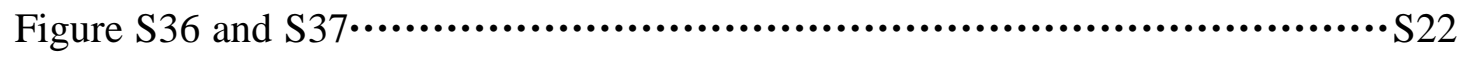


Figure S38 and S39

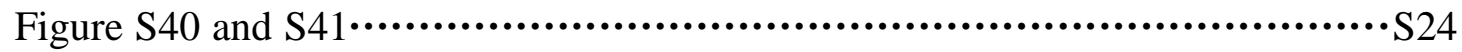

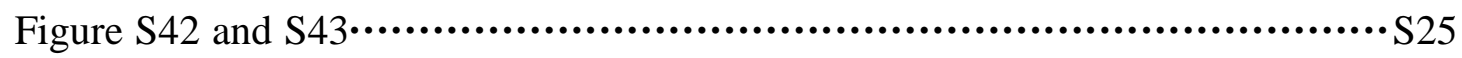

Figure S44 and S45

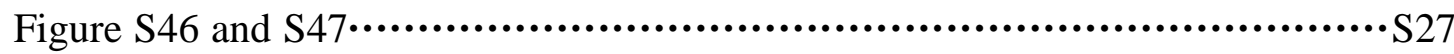

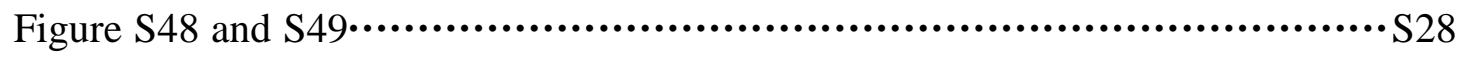

Figure S50 and S51

Table S1 and Table S2

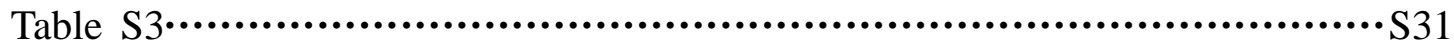

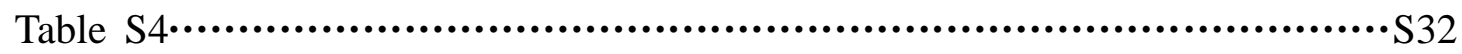

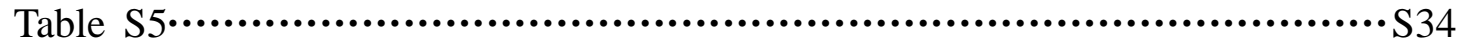



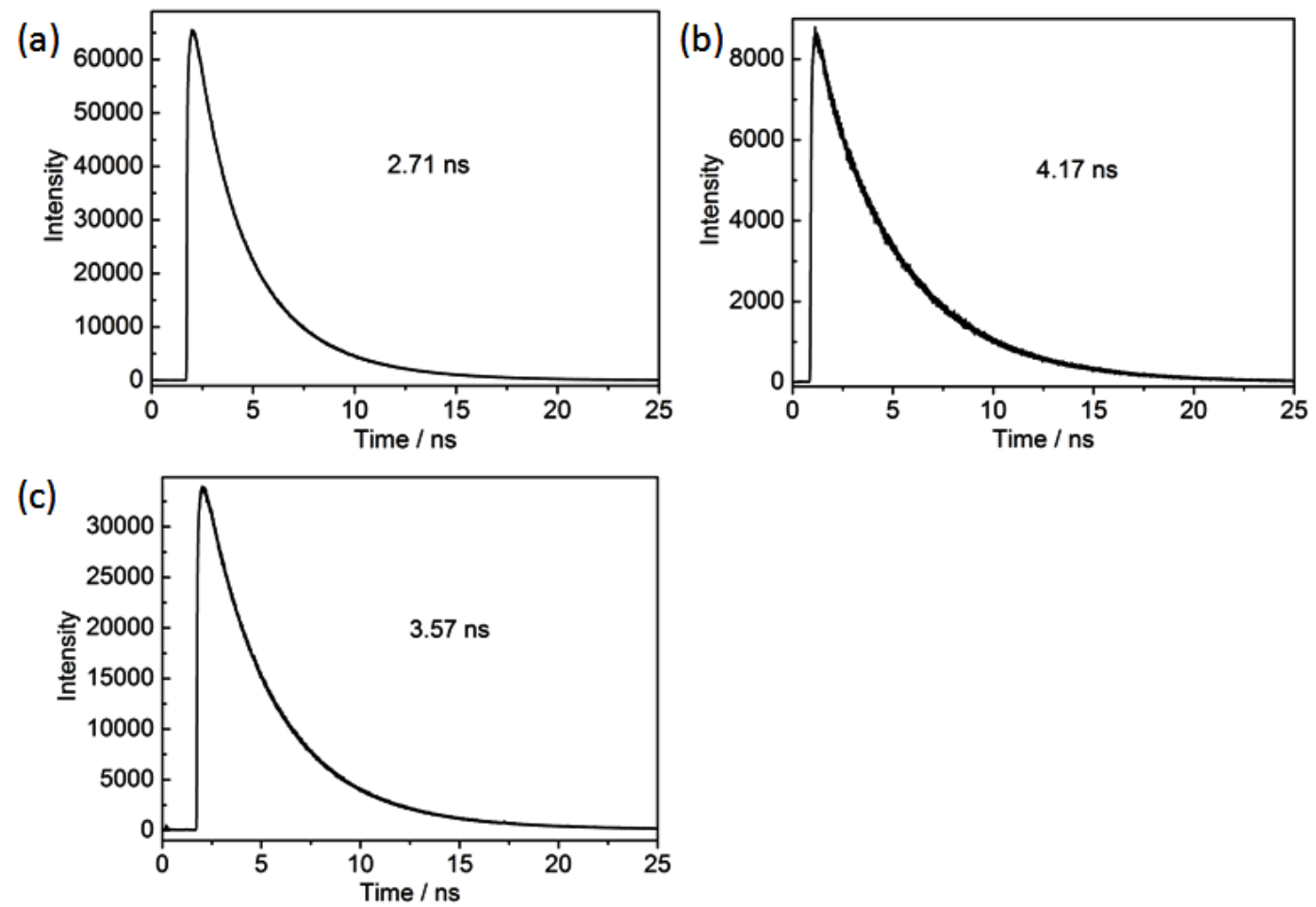

Figure S1 Fluorescence decay of molecules 6 (a), 9a (b) and 9b (c) in degassed dichloromethane. 

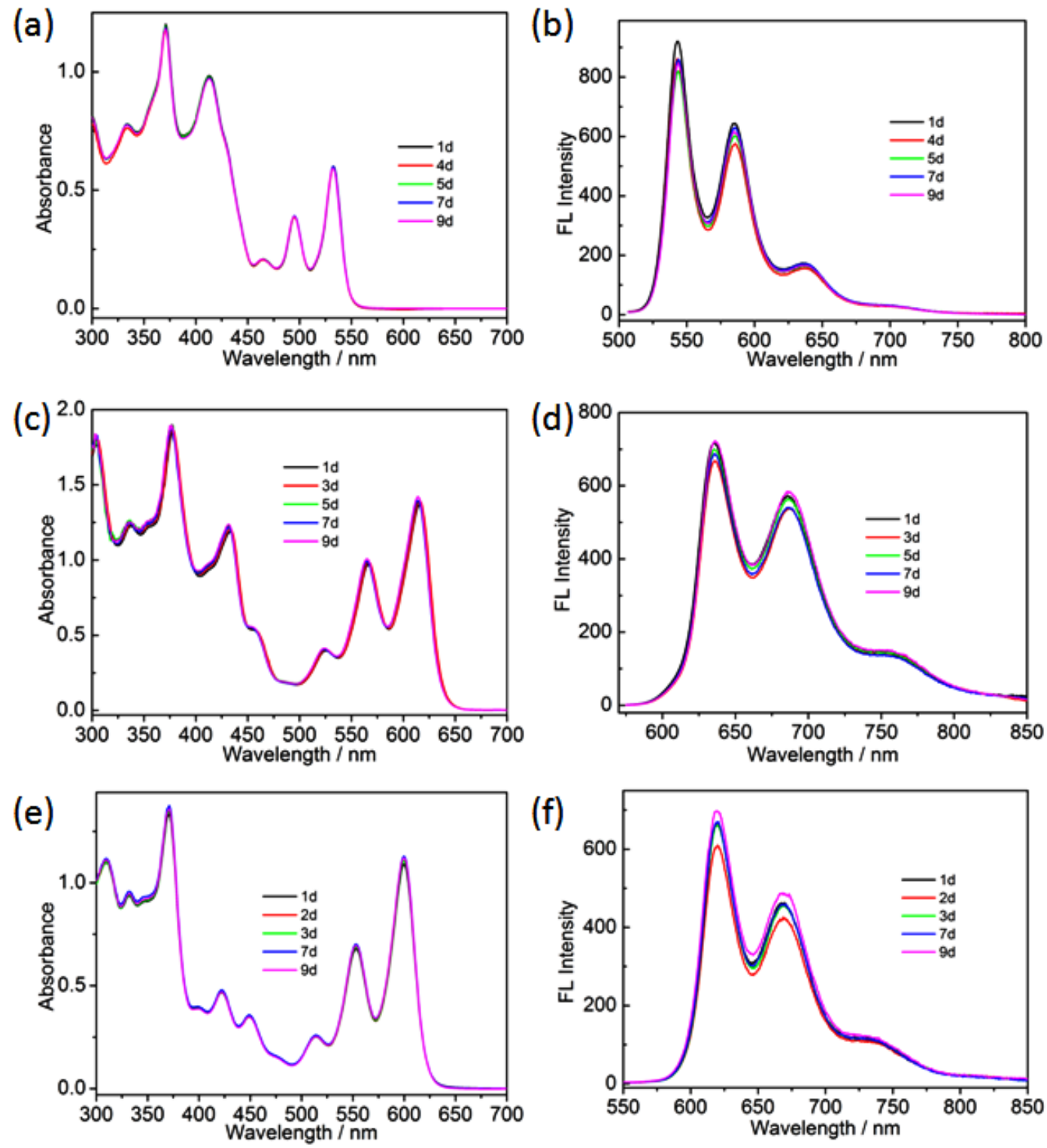

Figure S2 Changes of UV-vis absorption and emission spectra 6 (a)/(b), 9a (c)/(d) and 9b (e)/(f) in toluene under ambient light and air conditions. 

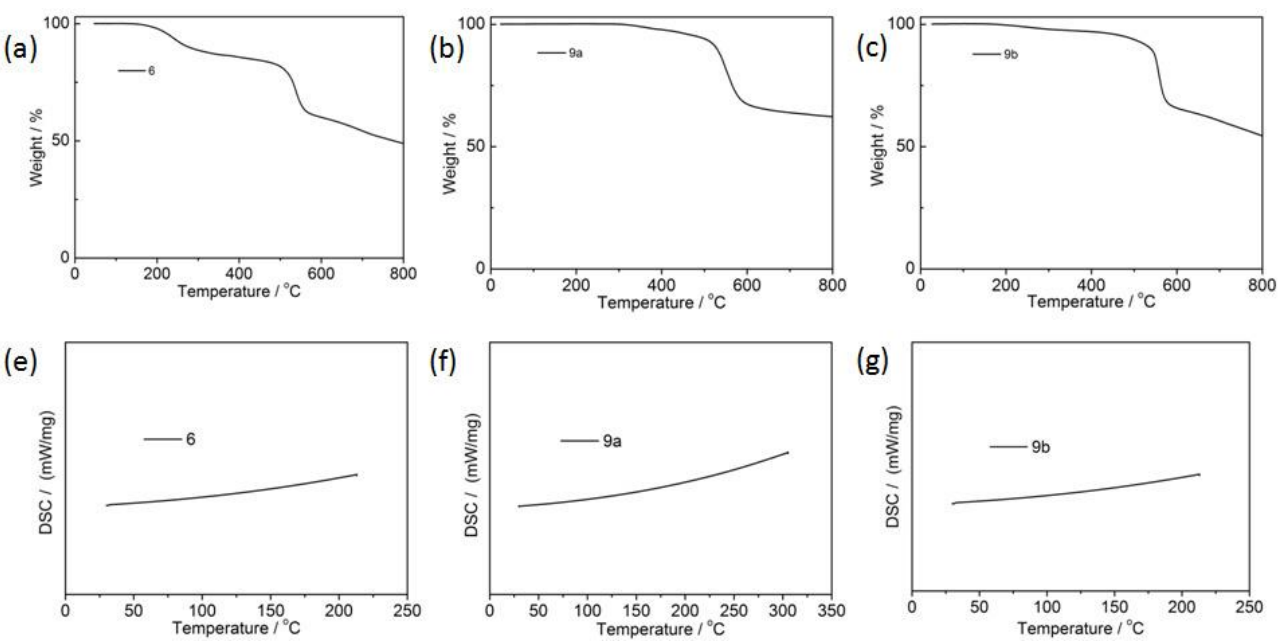

Figure S3 TGA curves of $\mathbf{6}(\mathrm{a}), \mathbf{9 a}(\mathrm{b})$ and $9 \mathbf{b}$ (c) under nitrogen with a heating rate of $10{ }^{\circ} \mathrm{C} / \mathrm{min}$. DSC thermal profiles of $\mathbf{6}(\mathrm{e}), \mathbf{9 a}(\mathrm{f})$ and $\mathbf{9 b}(\mathrm{g})$ under nitrogen with a heating rate of $10{ }^{\circ} \mathrm{C} / \mathrm{min}$. 


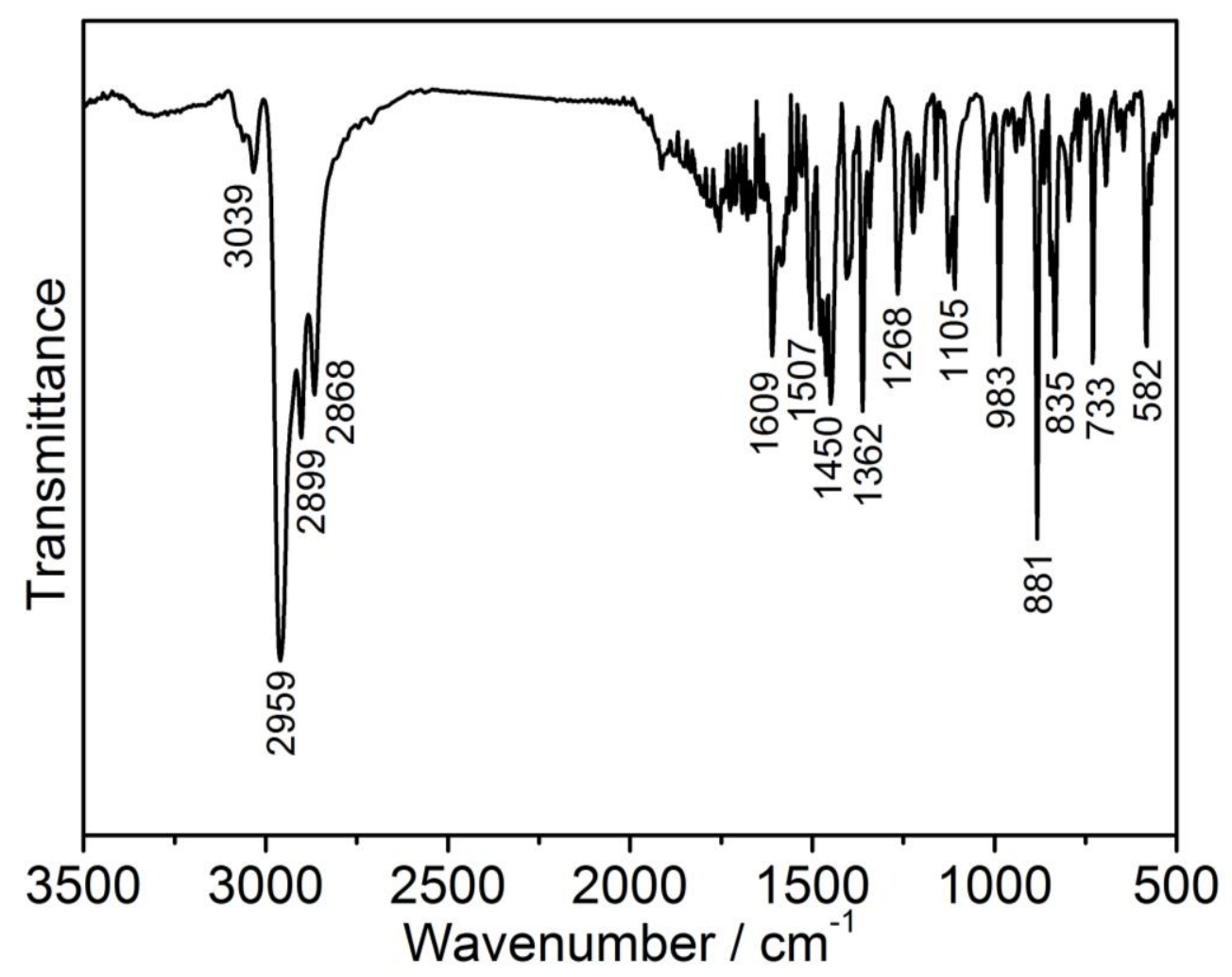

Figure S4 FT-IR spectrum of 3 .

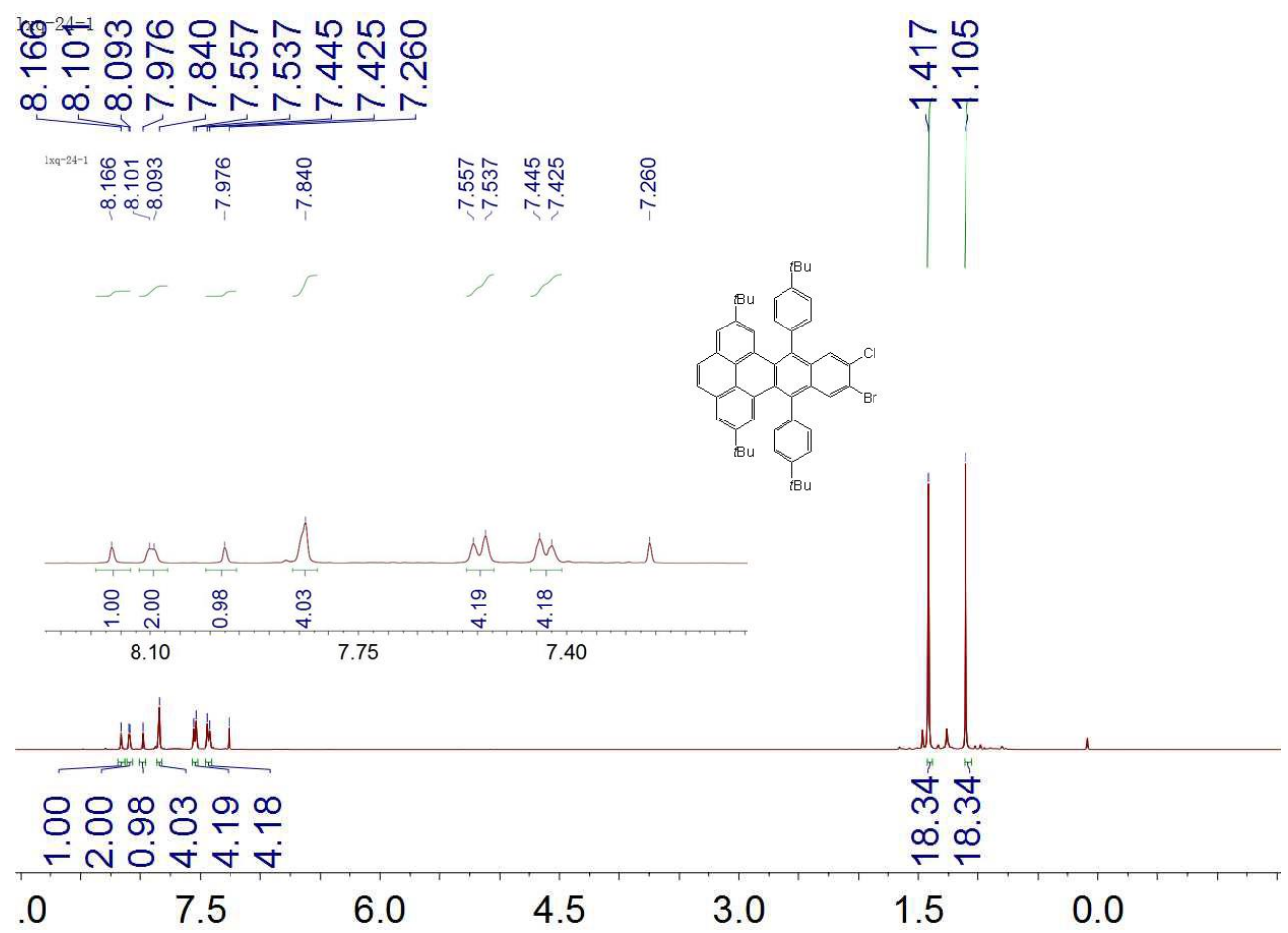

Figure $\mathrm{S} 5{ }^{1} \mathrm{H}$ NMR spectrum of 3 in $\mathrm{CDCl}_{3}(400 \mathrm{MHz})$. 


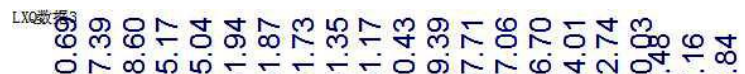

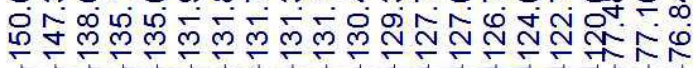

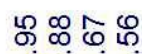

लें लुलं

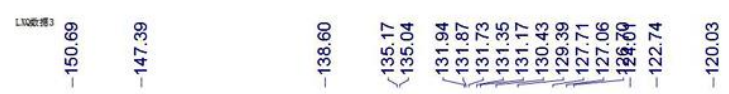

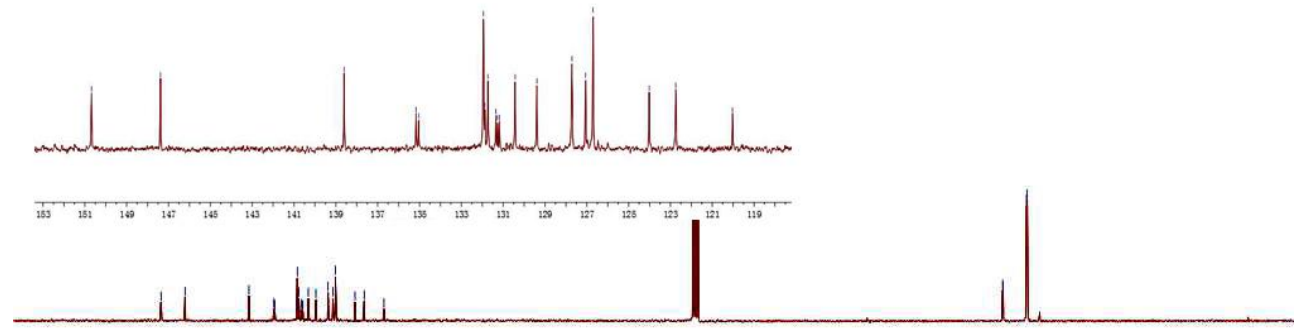

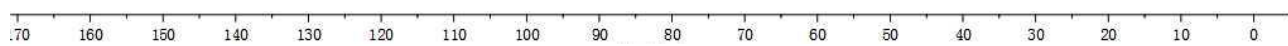

Figure $\mathrm{S} 6{ }^{13} \mathrm{C}\{1 \mathrm{H}\}$ NMR spectrum of $\mathbf{3}$ in $\mathrm{CDCl}_{3}(100 \mathrm{MHz})$.

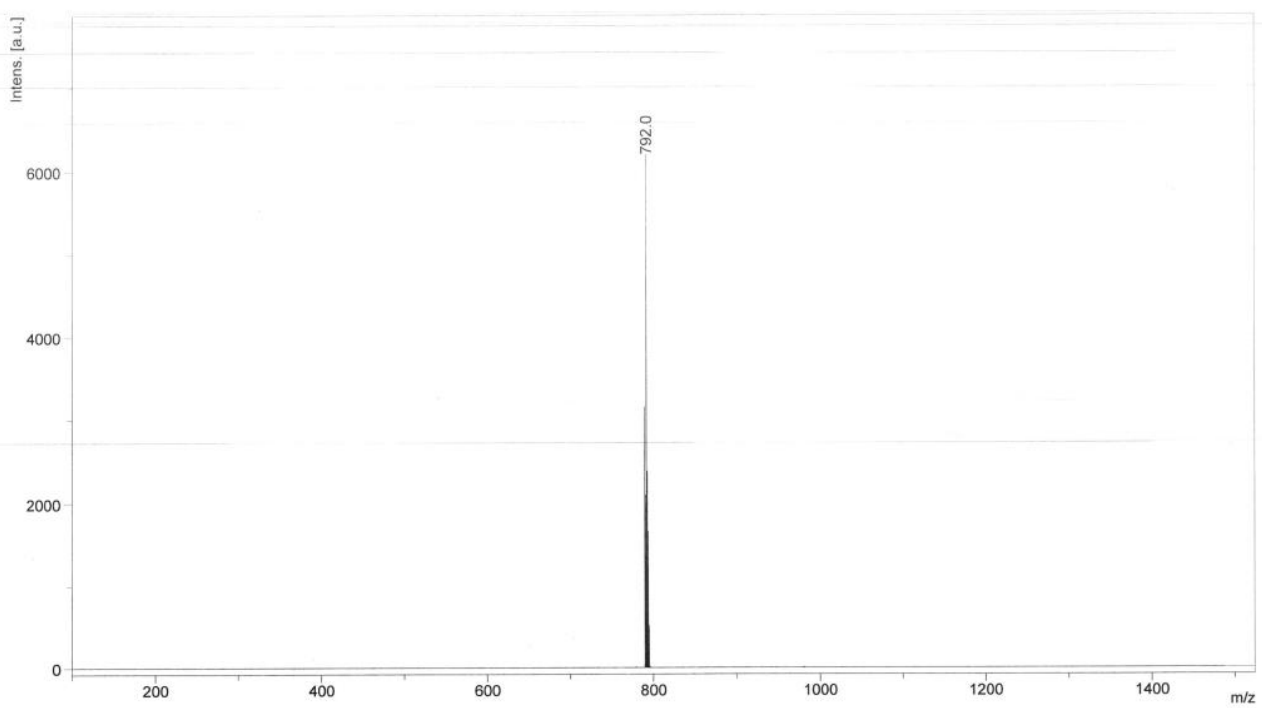

Figure S7 MALDI-TOF spectrum of 3. 


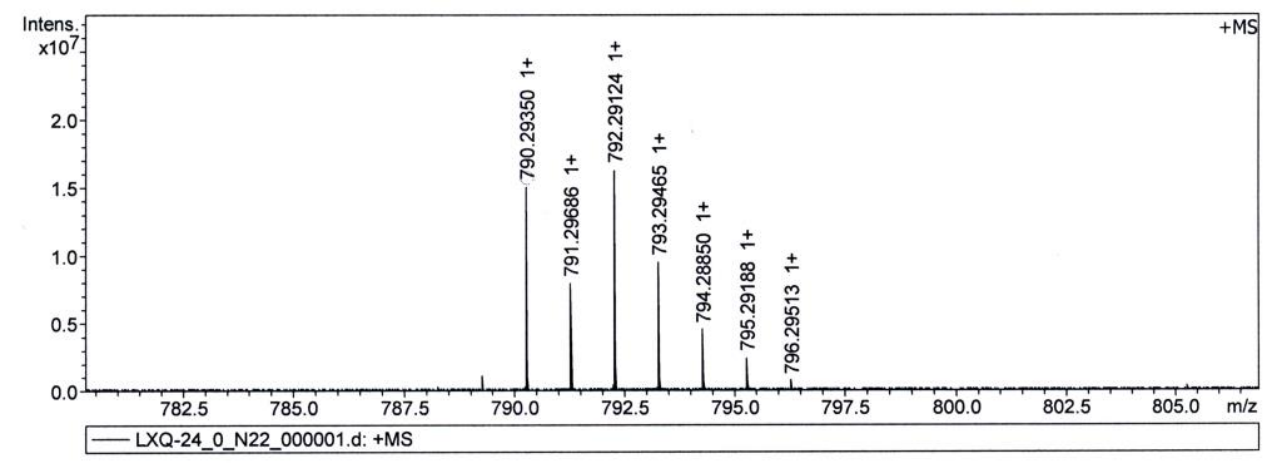

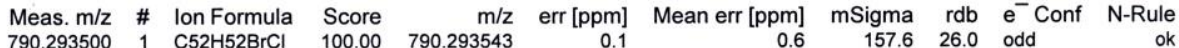

Figure S8 HR-MS spectrum of 3.

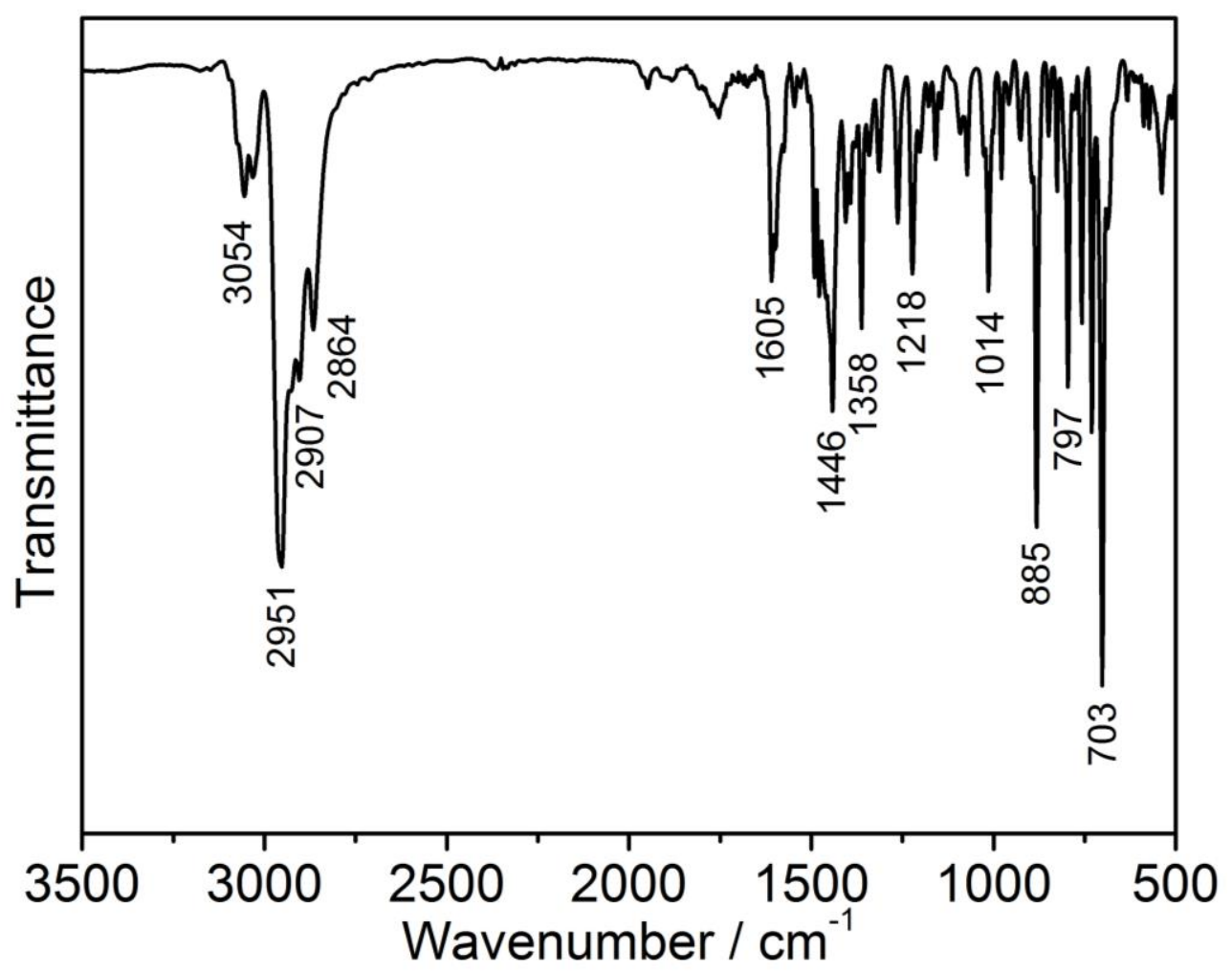

Figure S9 FT-IR spectrum of $\mathbf{5}$. 


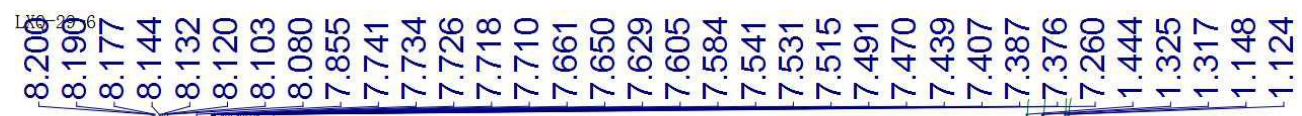

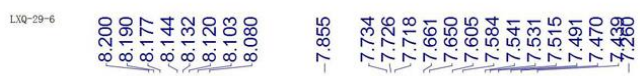
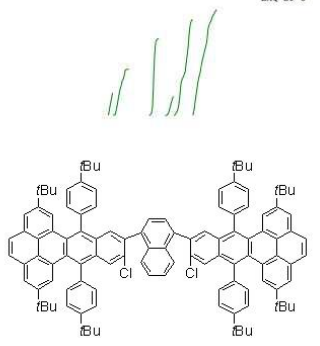

the colvowien

$$
\text { 1Bu }
$$

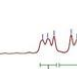

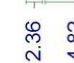

8.30

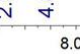

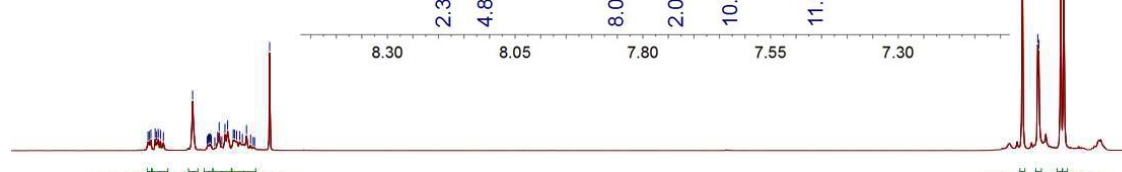

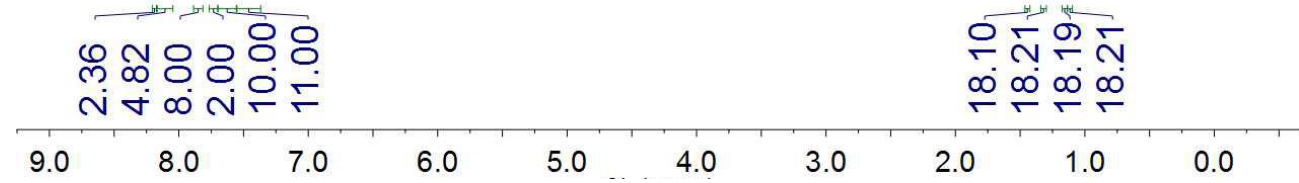

Figure $\mathrm{S} 10{ }^{1} \mathrm{H}$ NMR spectrum of 5 in $\mathrm{CDCl}_{3}(400 \mathrm{MHz})$.

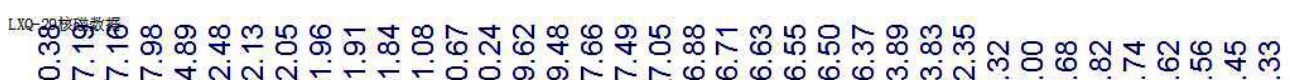

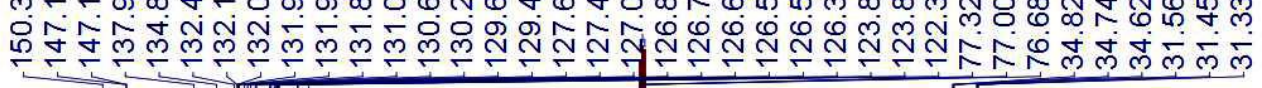

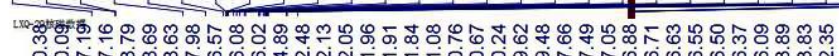

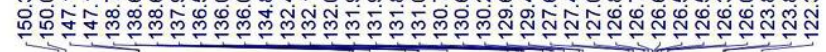

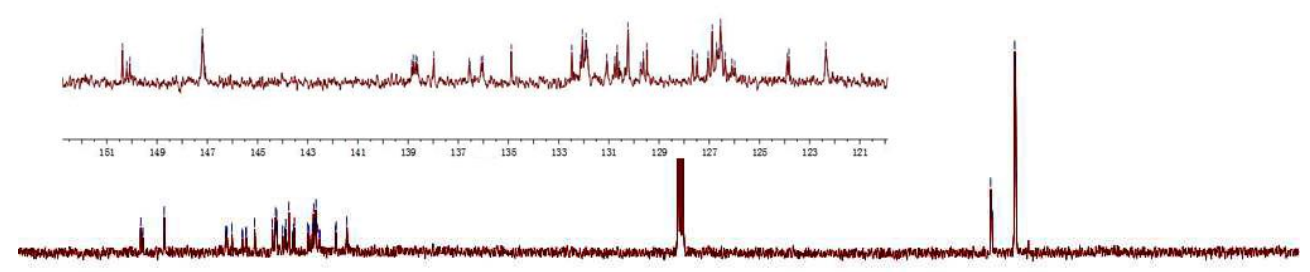

Figure $\mathrm{S} 11{ }^{13} \mathrm{C}\{1 \mathrm{H}\}$ NMR spectrum of 5 in $\mathrm{CDCl}_{3}(100 \mathrm{MHz})$. 


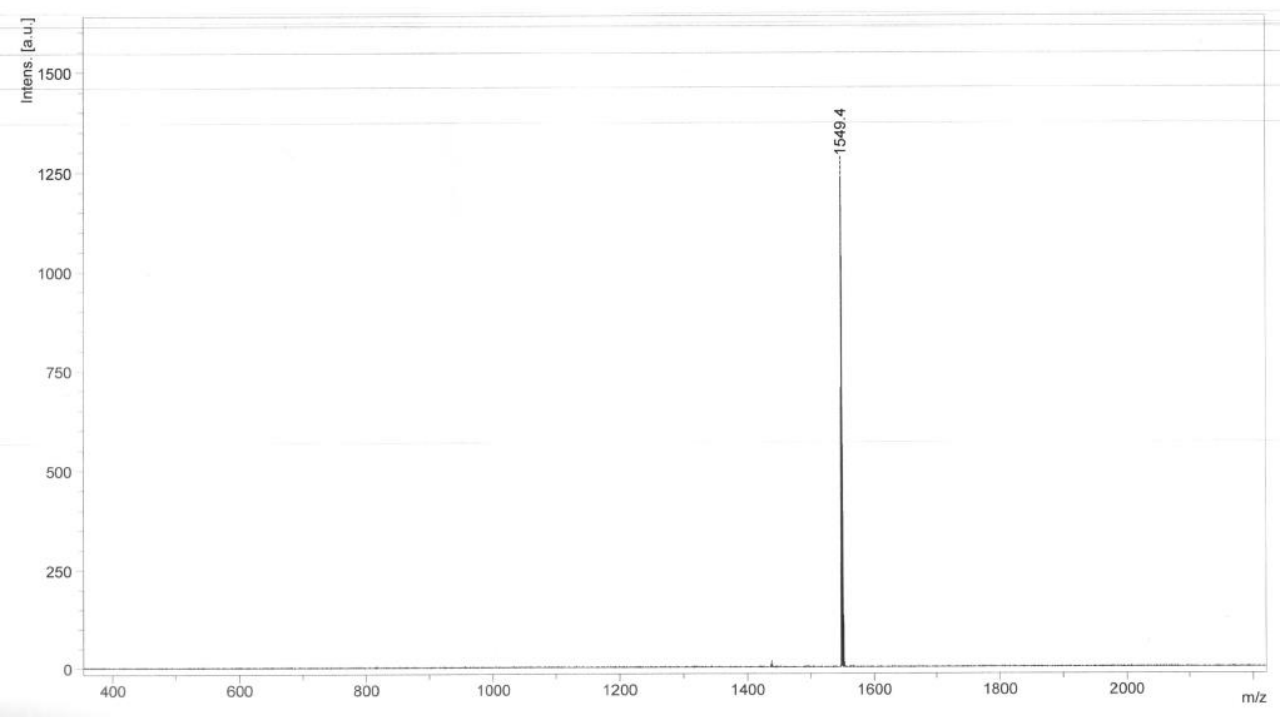

Figure S12 MALDI-TOF spectrum of 5.

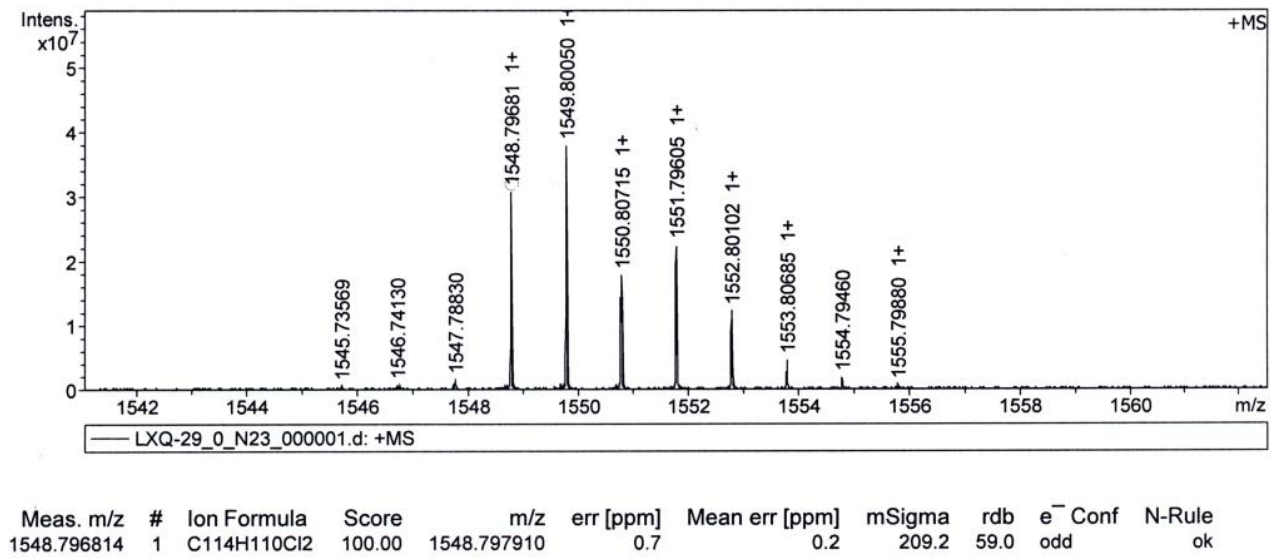

Figure S13 HR-MS spectrum of 5. 


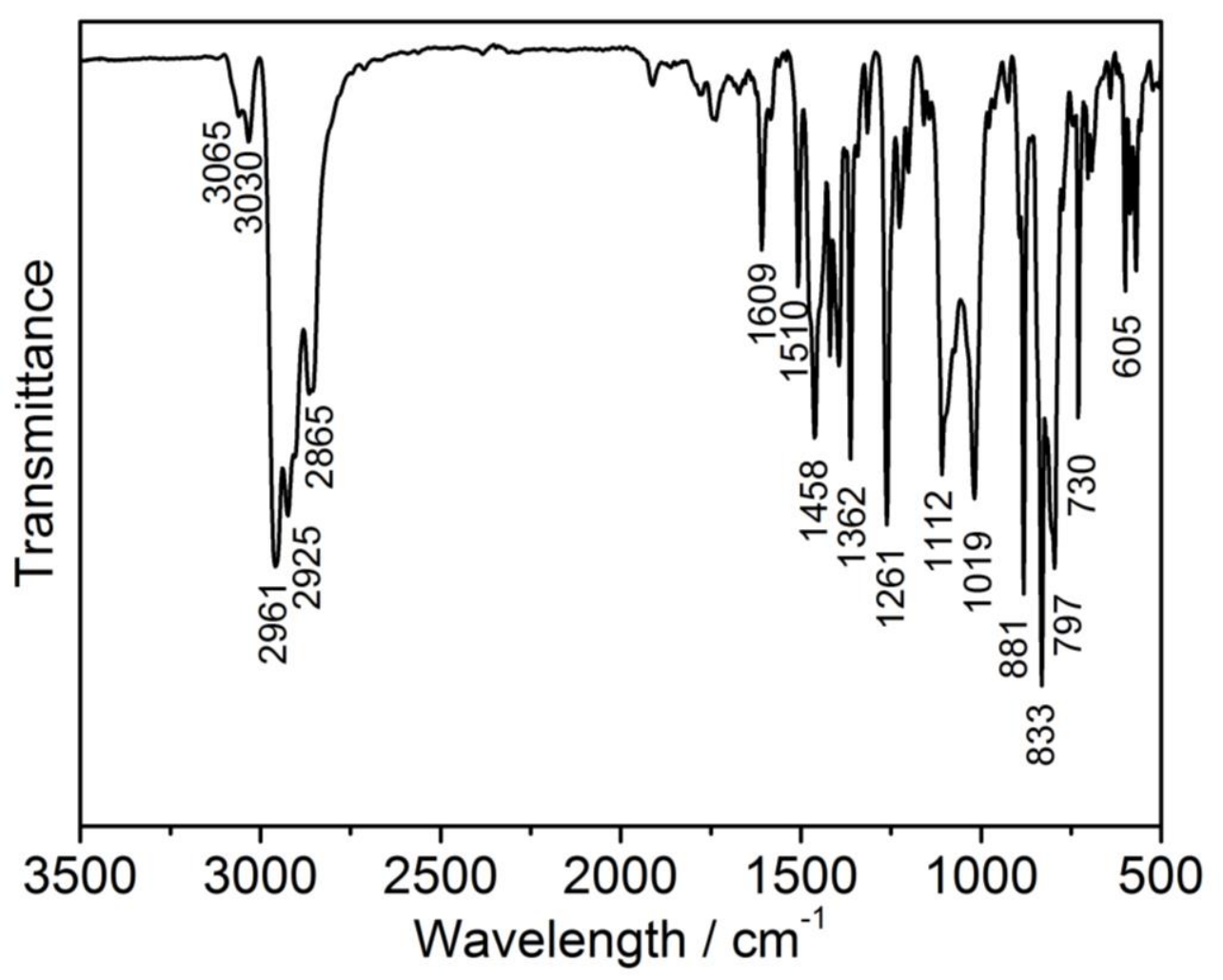

Figure S14 FT-IR spectrum of 6.

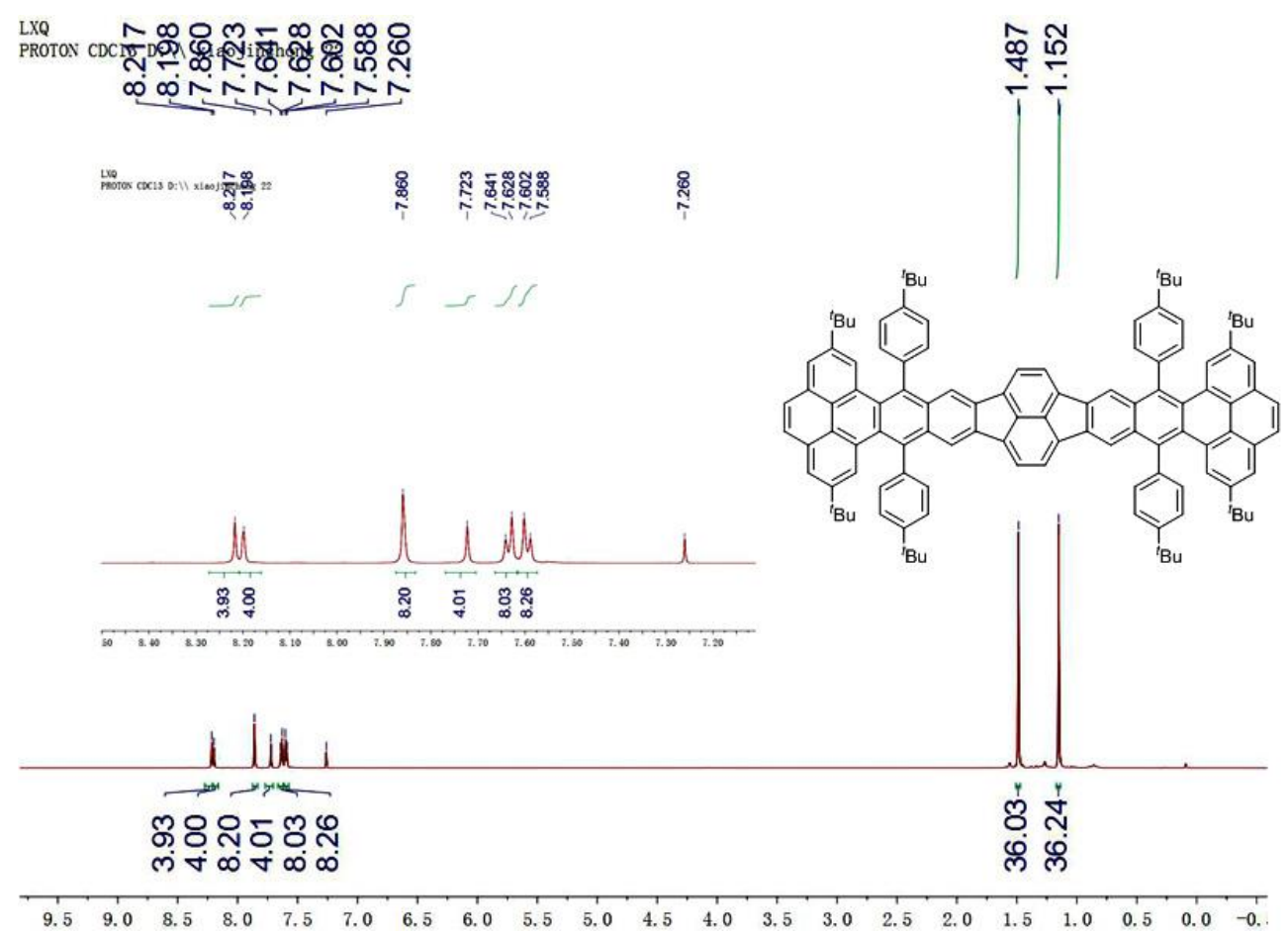

Figure S15 ${ }^{1} \mathrm{H}$ NMR spectrum of 6 in $\mathrm{CDCl}_{3}(600 \mathrm{MHz})$. 


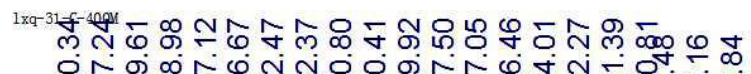

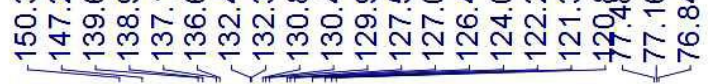

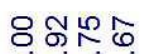

फुल्लें்

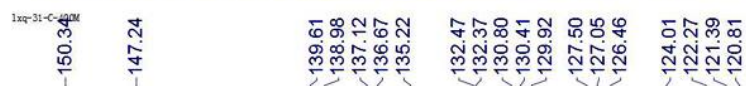
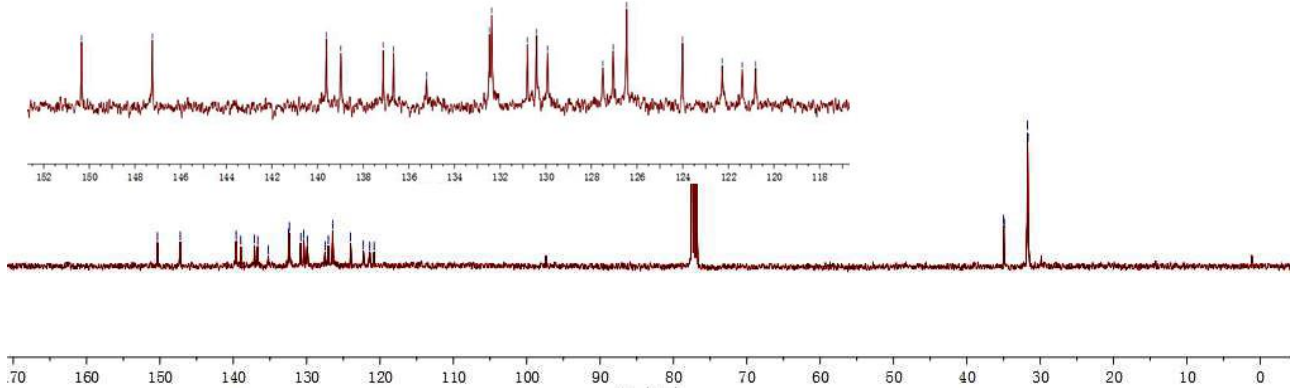

Figure $\mathrm{S} 16{ }^{13} \mathrm{C}\{1 \mathrm{H}\} \mathrm{NMR}$ spectrum of 6 in $\mathrm{CDCl}_{3}(100 \mathrm{MHz})$.

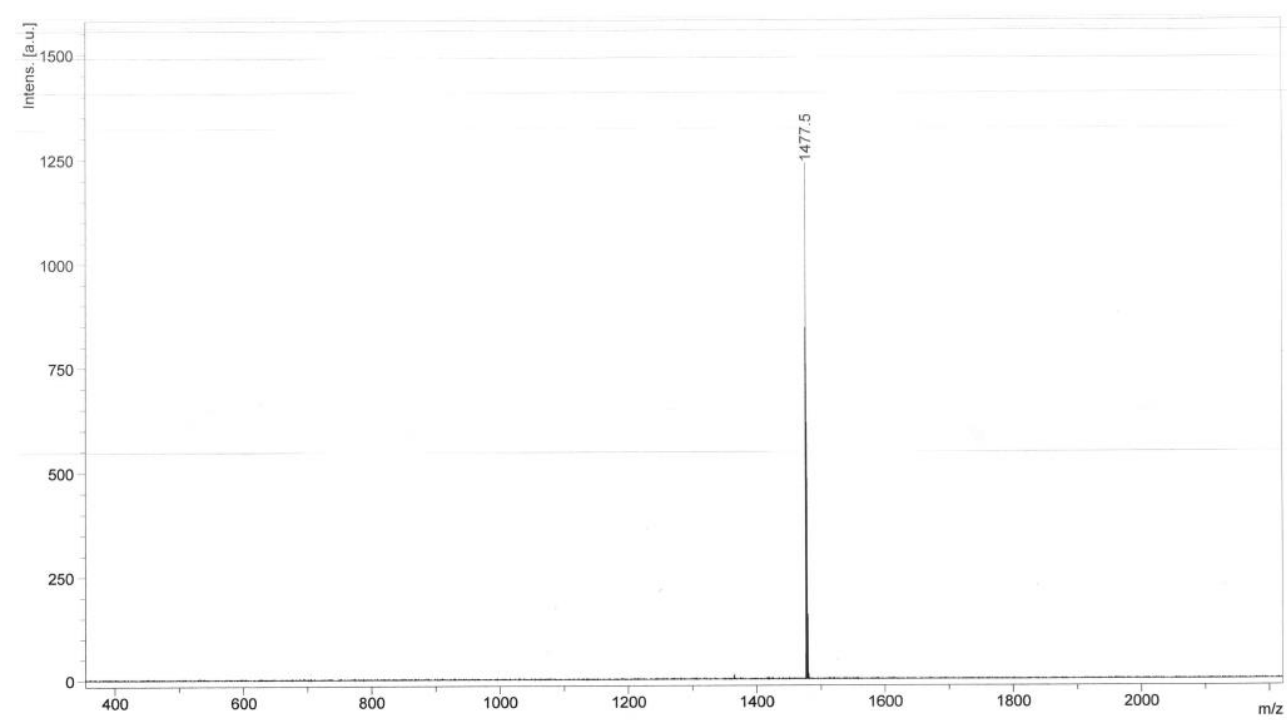

Figure S17 MALDI-TOF spectrum of 6. 


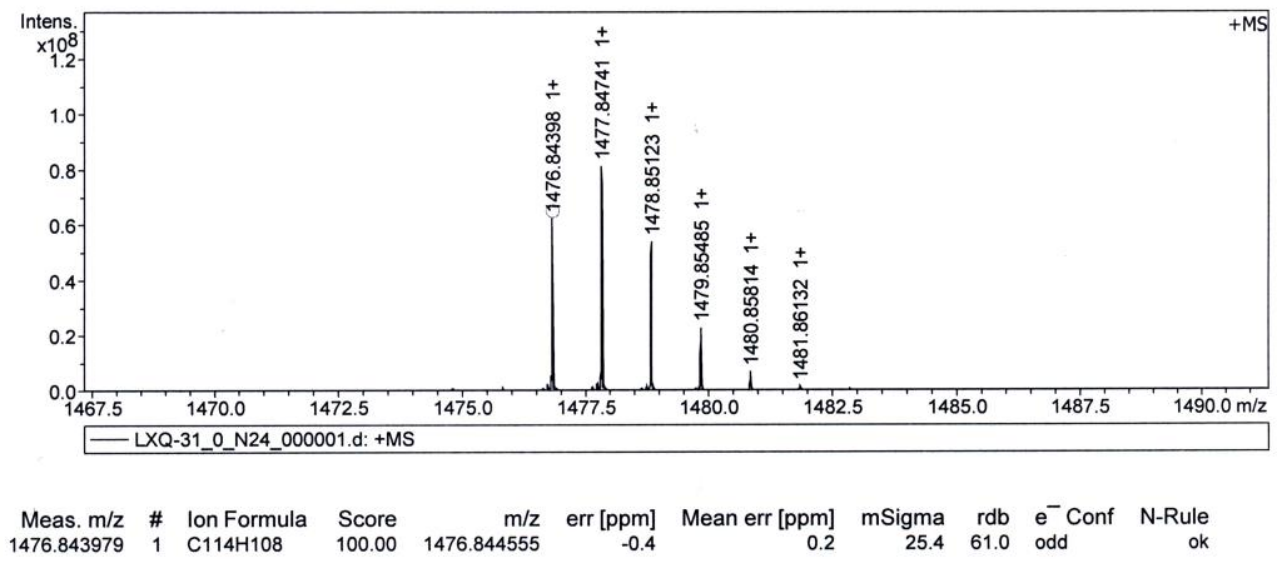

Figure S18 HR-MS spectrum of 6.

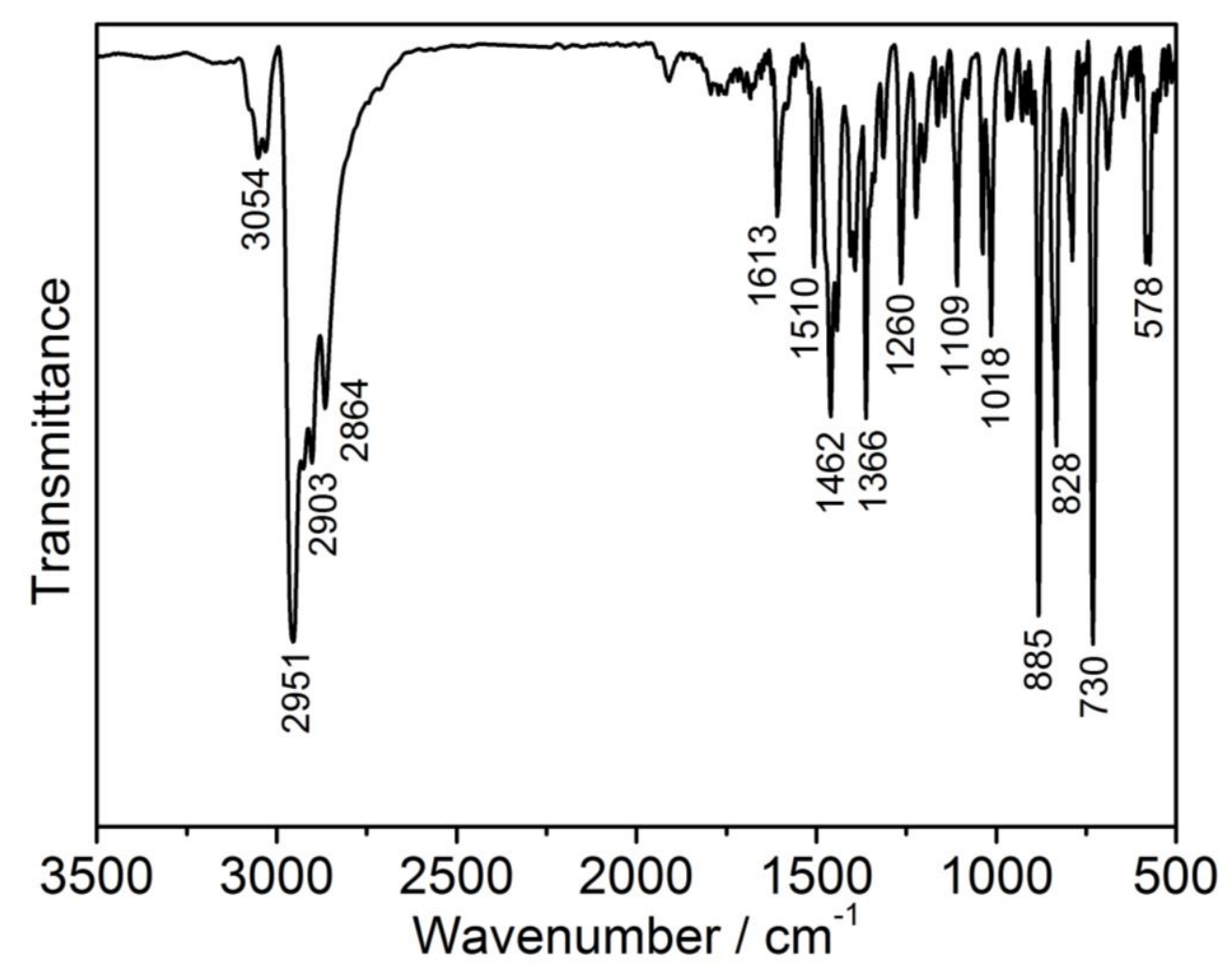

Figure S19 FT-IR spectrum of 8. 


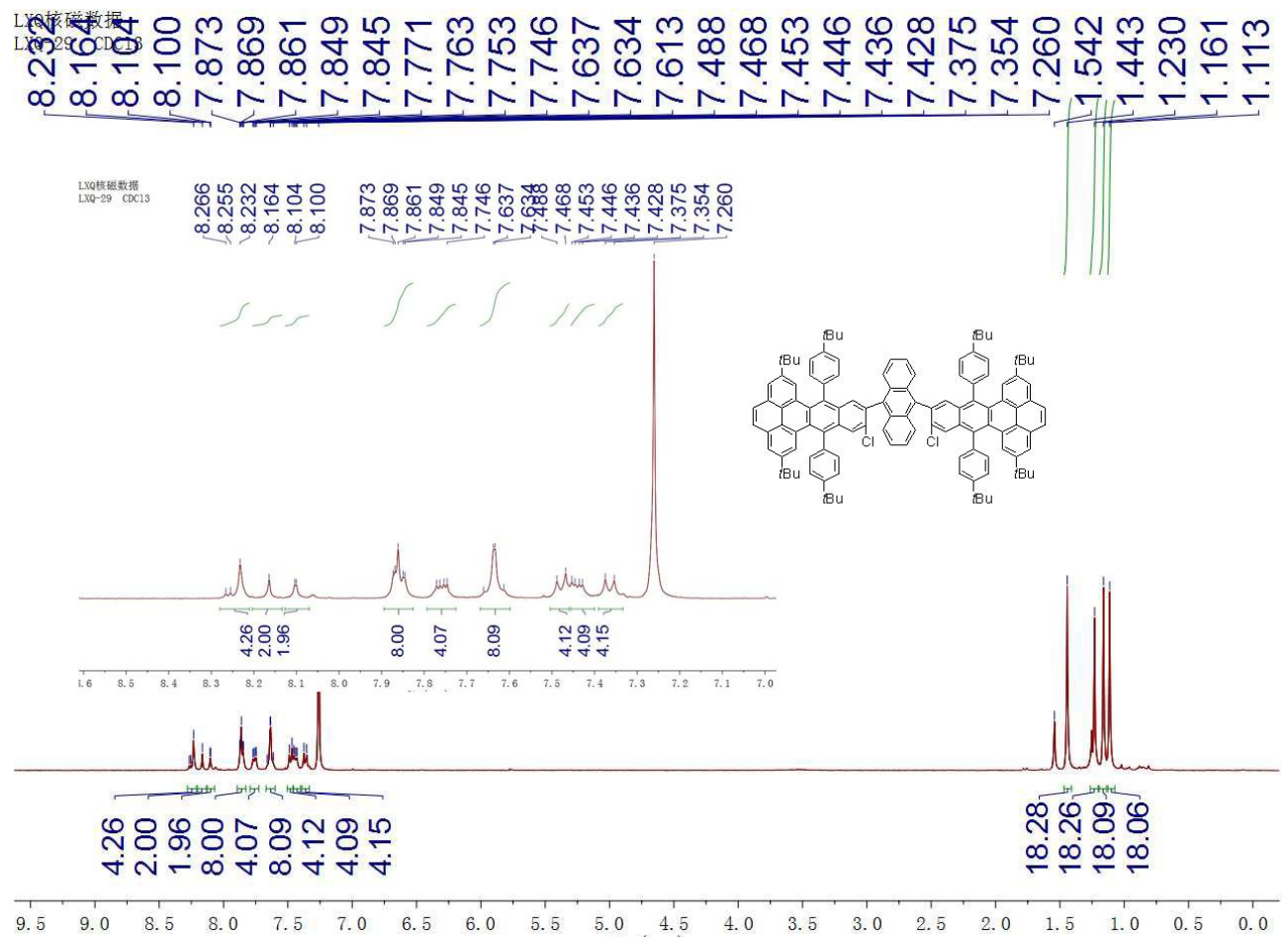

Figure S20 ${ }^{1} \mathrm{H}$ NMR spectrum of 8 in $\mathrm{CDCl}_{3}(400 \mathrm{MHz})$.

एक-

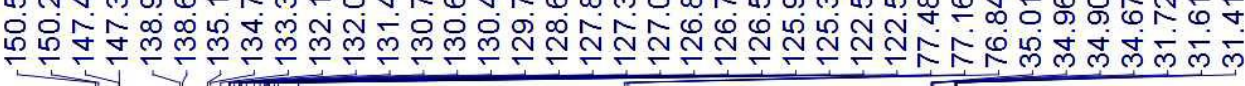

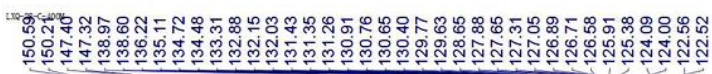

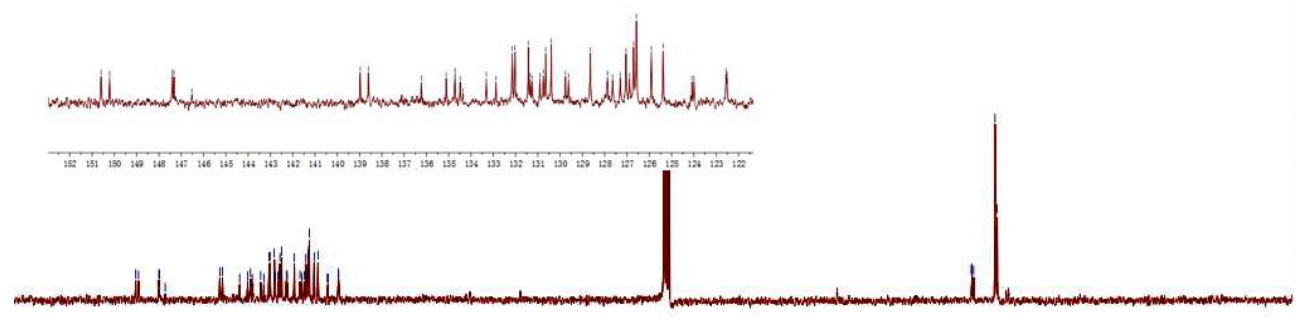

Figure $\mathrm{S} 21{ }^{13} \mathrm{C}$ NMR spectrum of $\mathbf{8}$ in $\mathrm{CDCl}_{3}(100 \mathrm{MHz})$. 


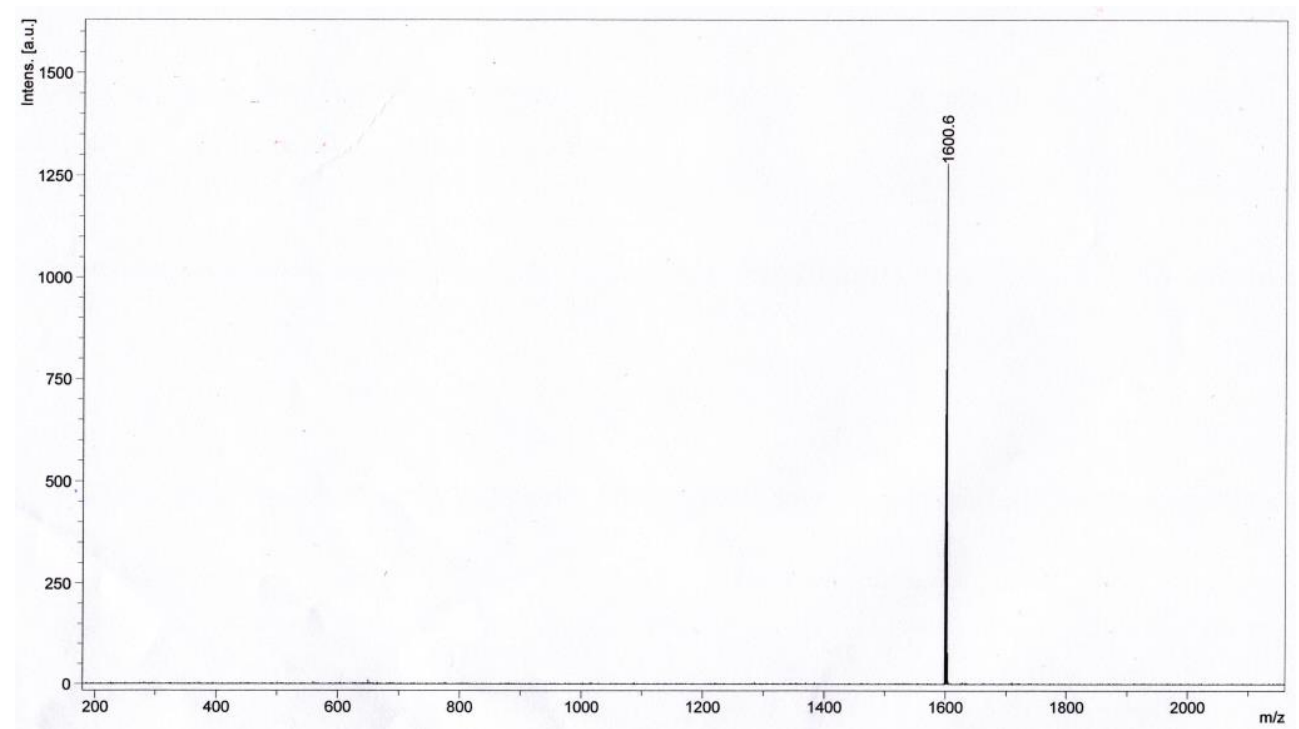

Figure S22 MALDI-TOF spectrum of 8 .

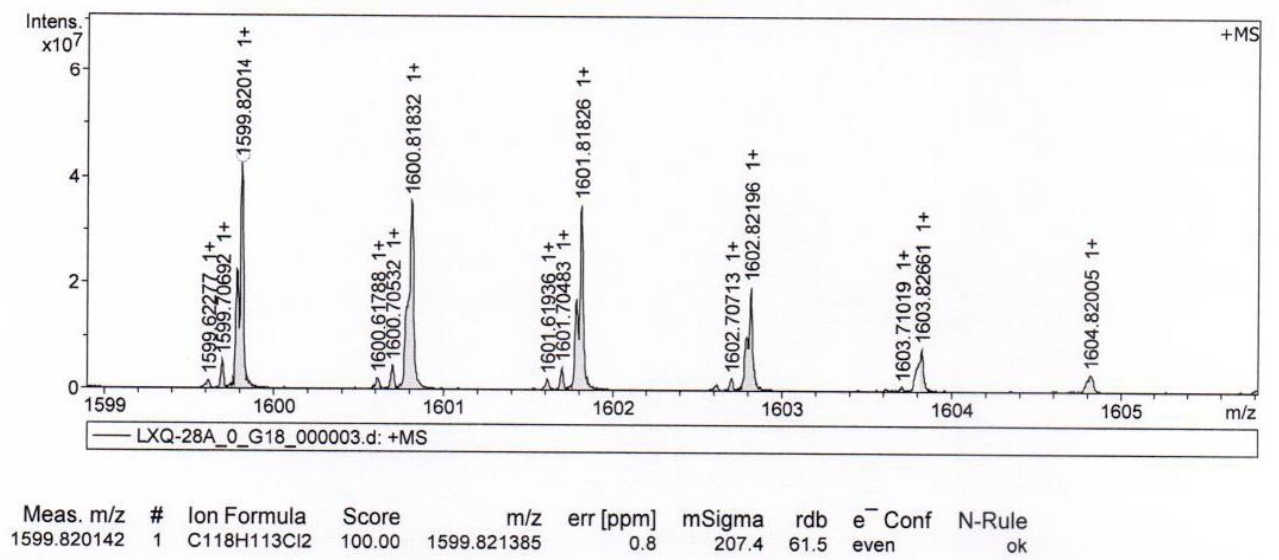

Figure S23 HR-MS of 8. 


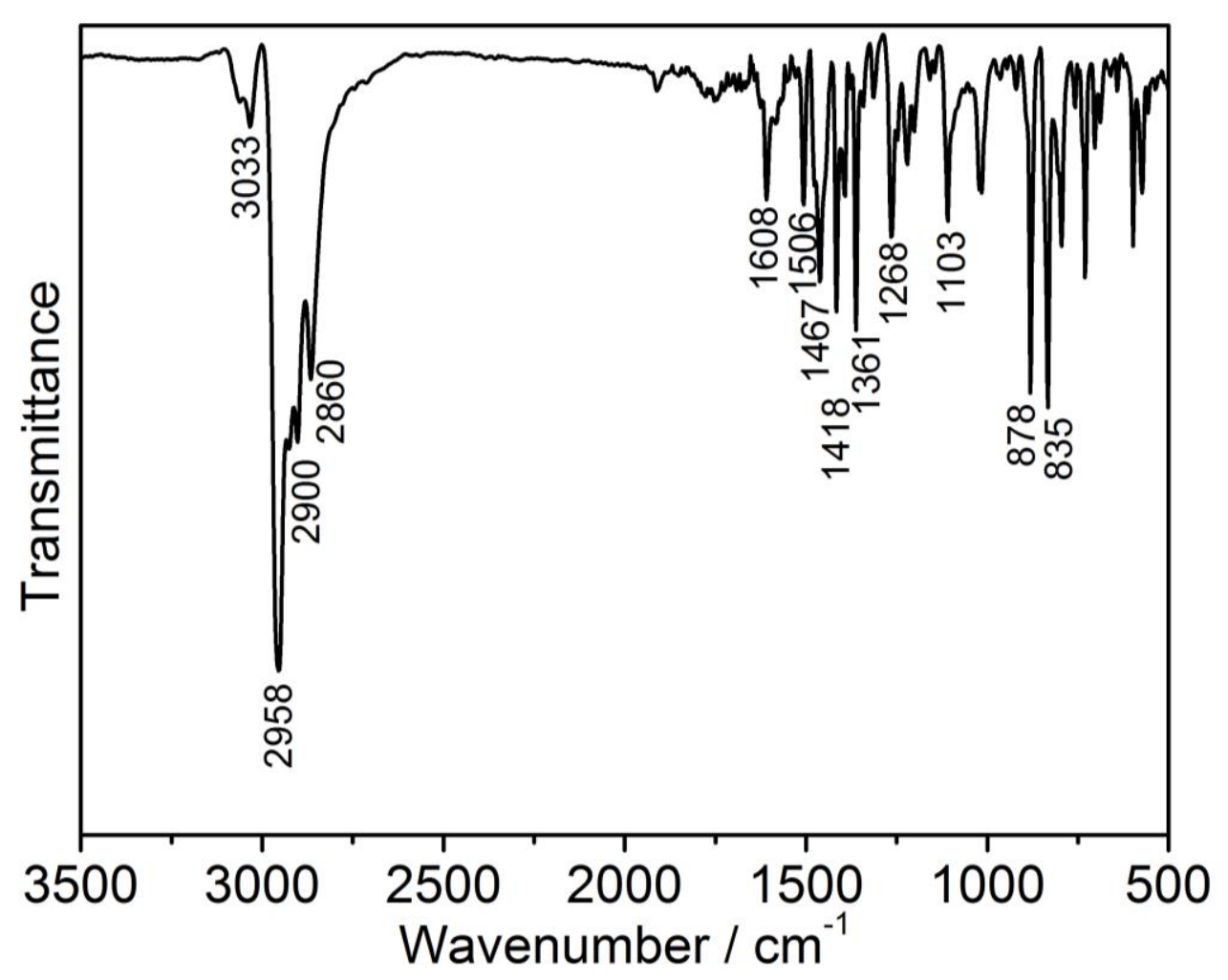

Figure S24 FT-IR spectrum of 9a.

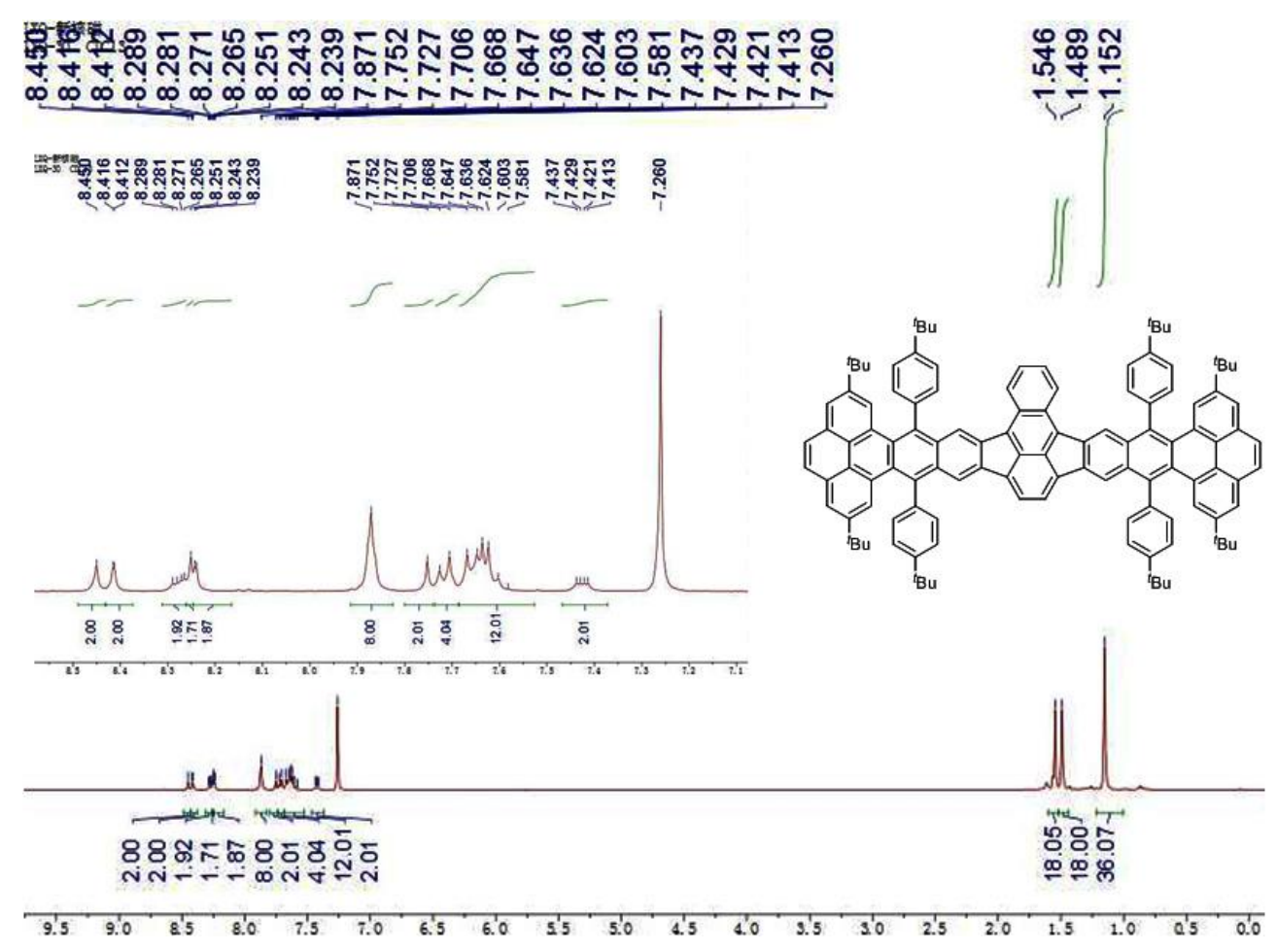

Figure S25 ${ }^{1} \mathrm{H}$ NMR spectrum of $9 \mathbf{a}$ in $\mathrm{CDCl}_{3}(400 \mathrm{MHz})$. 


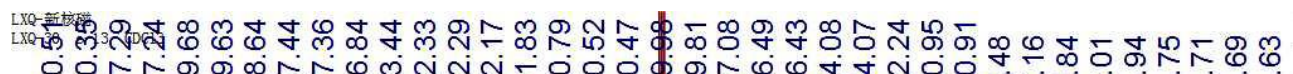

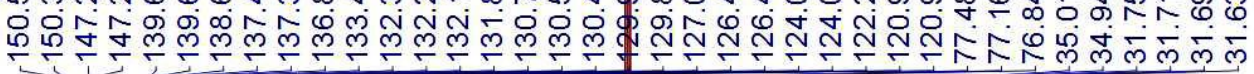

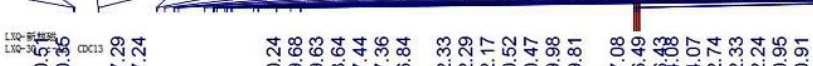

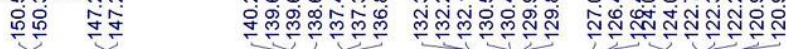
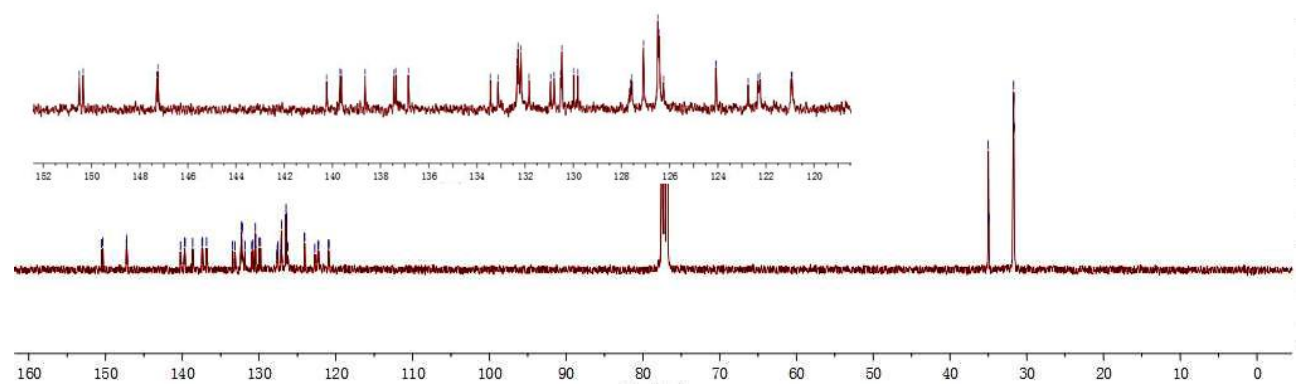

Figure S26 ${ }^{13} \mathrm{C}$ NMR spectrum of $\mathbf{9 a}$ in $\mathrm{CDCl}_{3}(100 \mathrm{MHz})$.

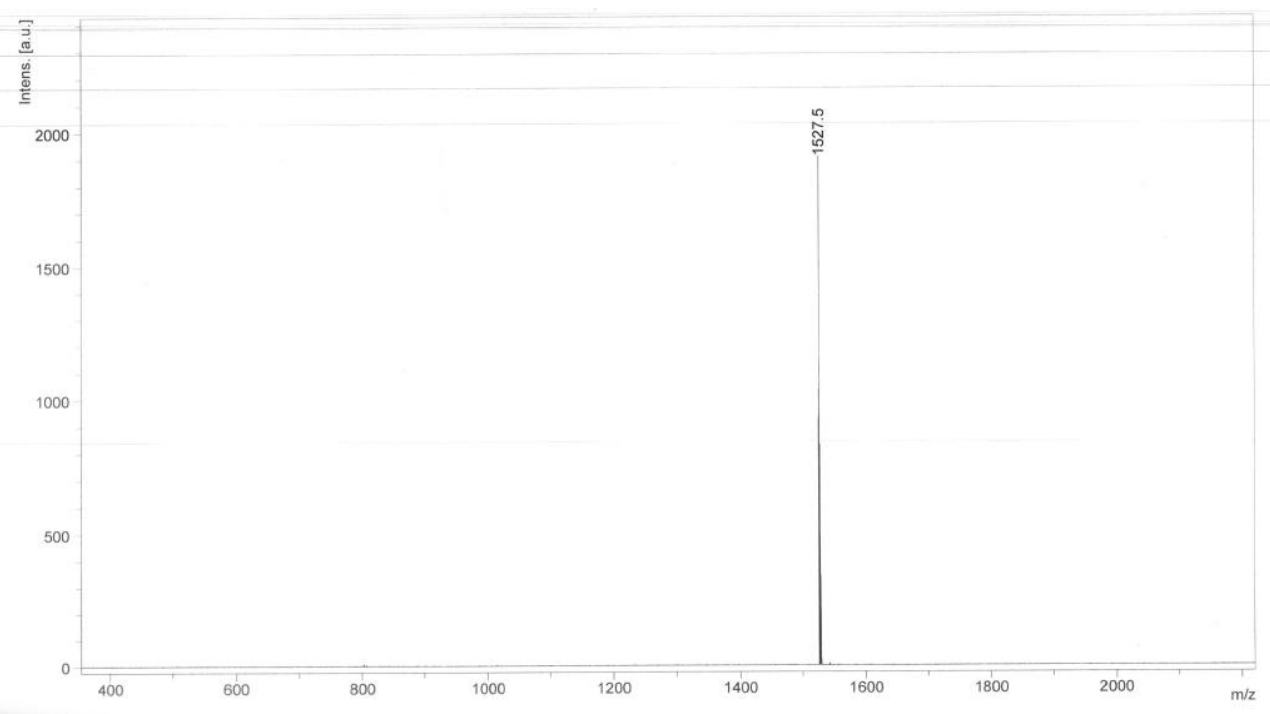

Figure S27 MALDI-TOF spectrum of 9a. 


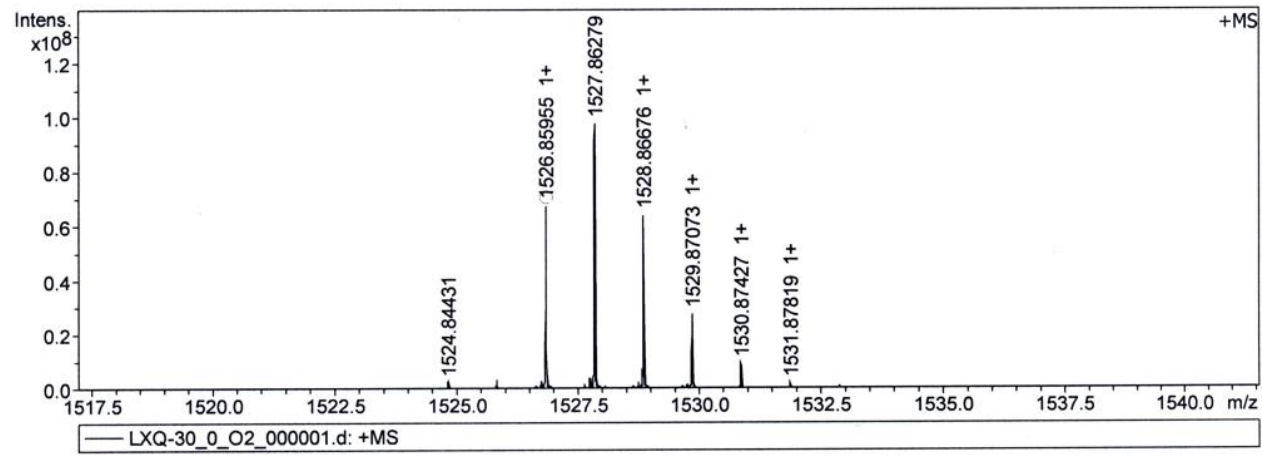

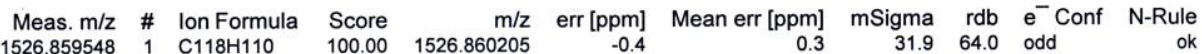

Figure S28 MALDI-TOF spectrum of 9a.

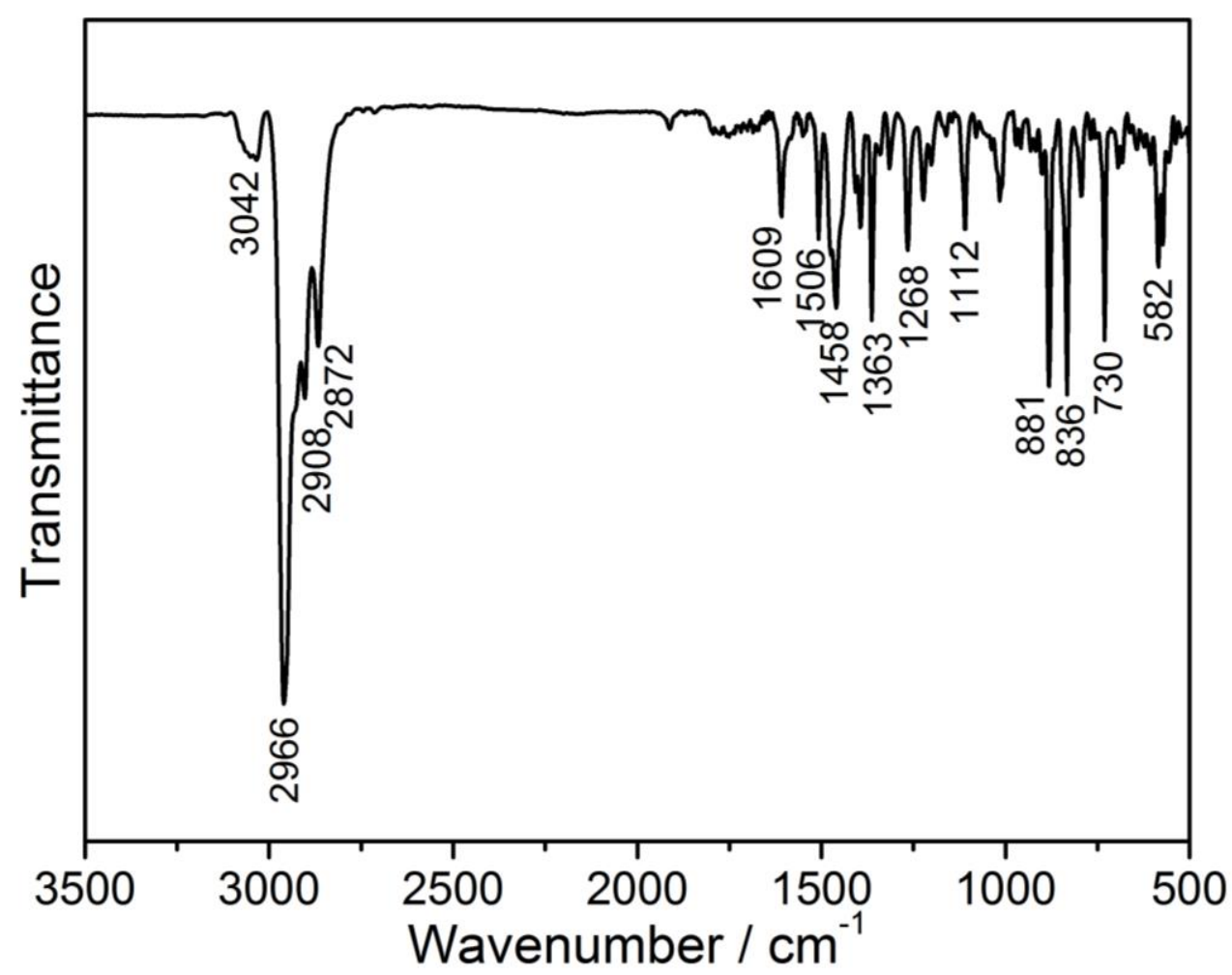

Figure S29 FT-IR spectrum of $\mathbf{1 1}$. 


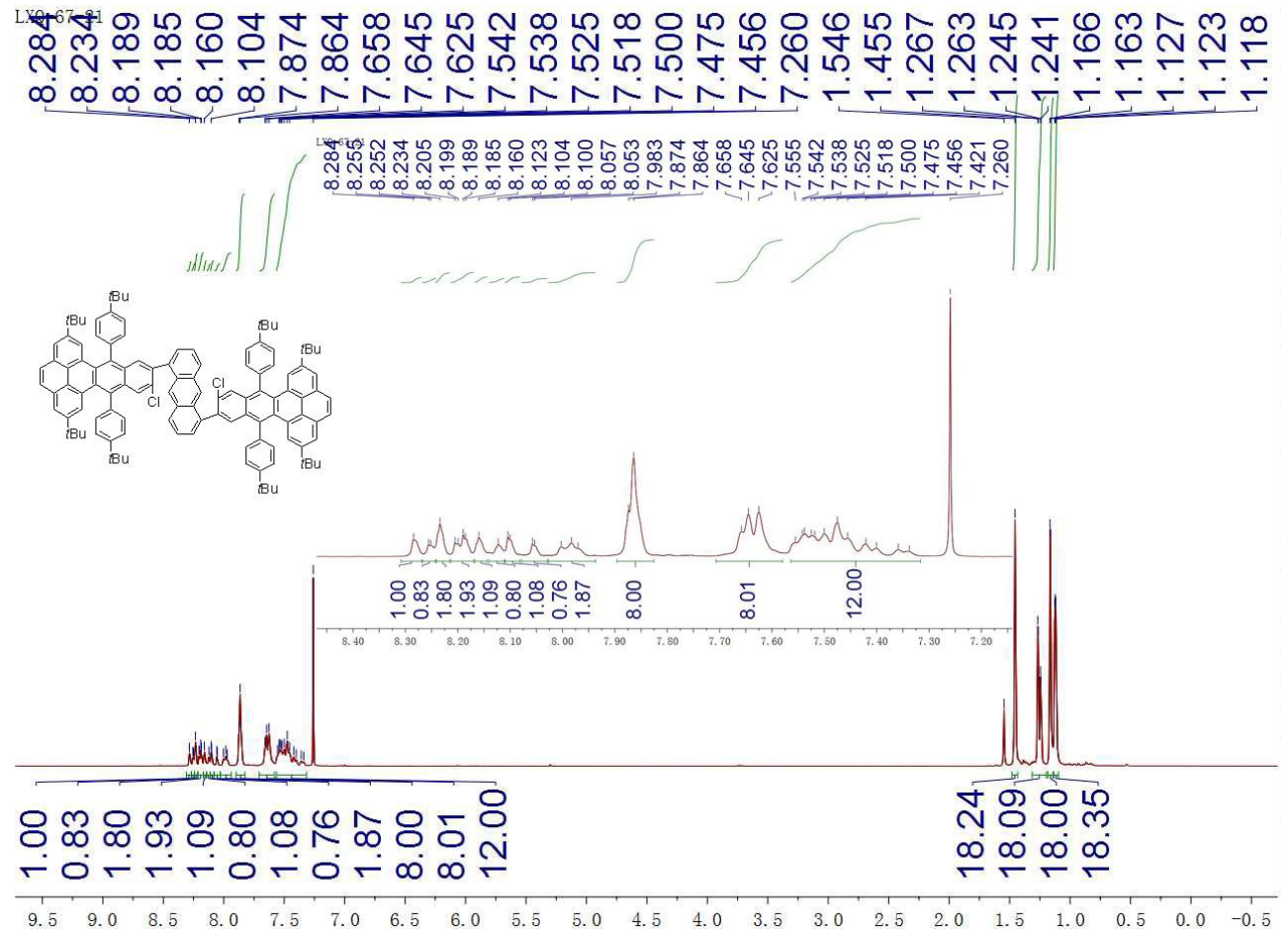

Figure $\mathrm{S} 30{ }^{1} \mathrm{H}$ NMR spectrum of 11 in $\mathrm{CDCl}_{3}(400 \mathrm{MHz})$.

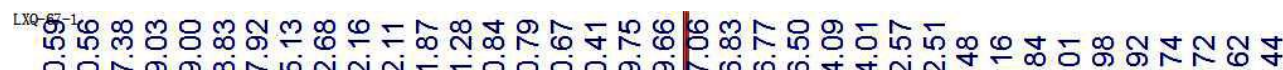
மํำ คํㅐำ

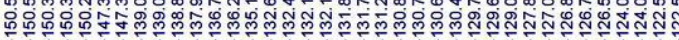
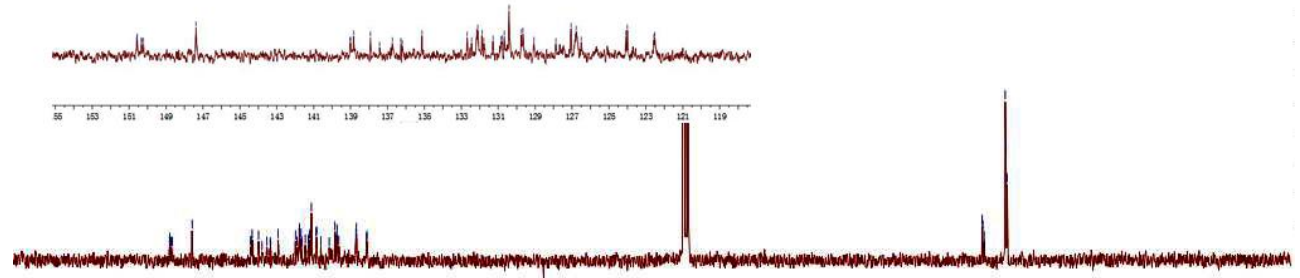

Figure $\mathrm{S} 31{ }^{13} \mathrm{C}$ NMR spectrum of $\mathbf{1 1}$ in $\mathrm{CDCl}_{3}(100 \mathrm{MHz})$. 


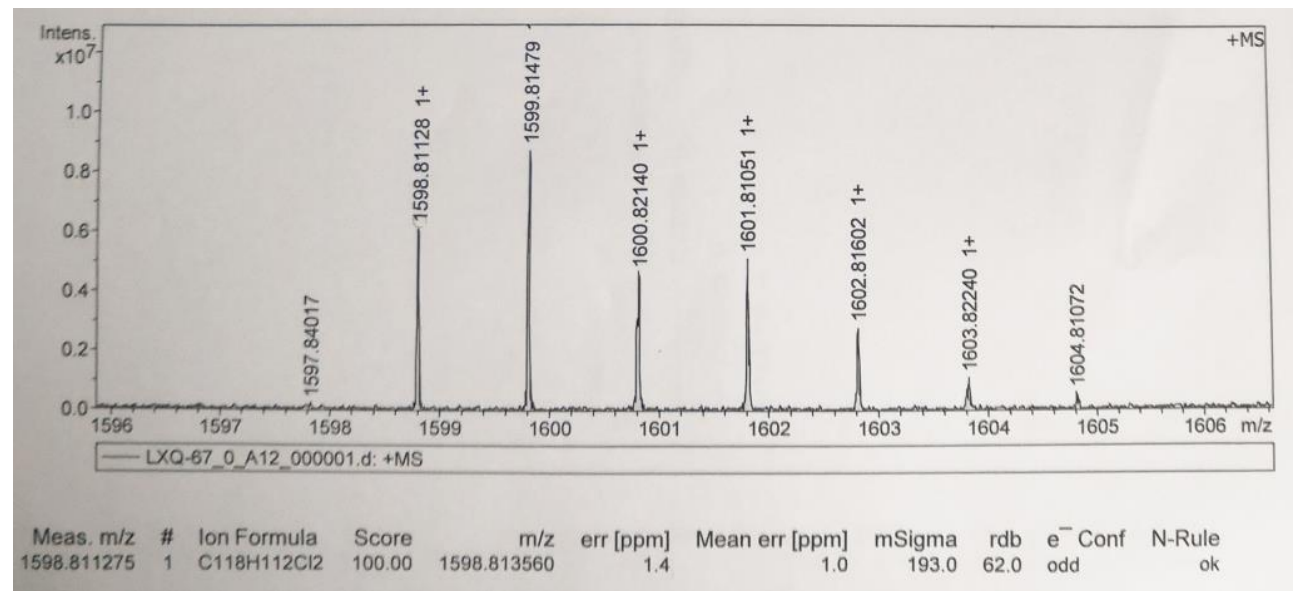

Figure S32 HR-MS spectrum of $\mathbf{1 1 .}$

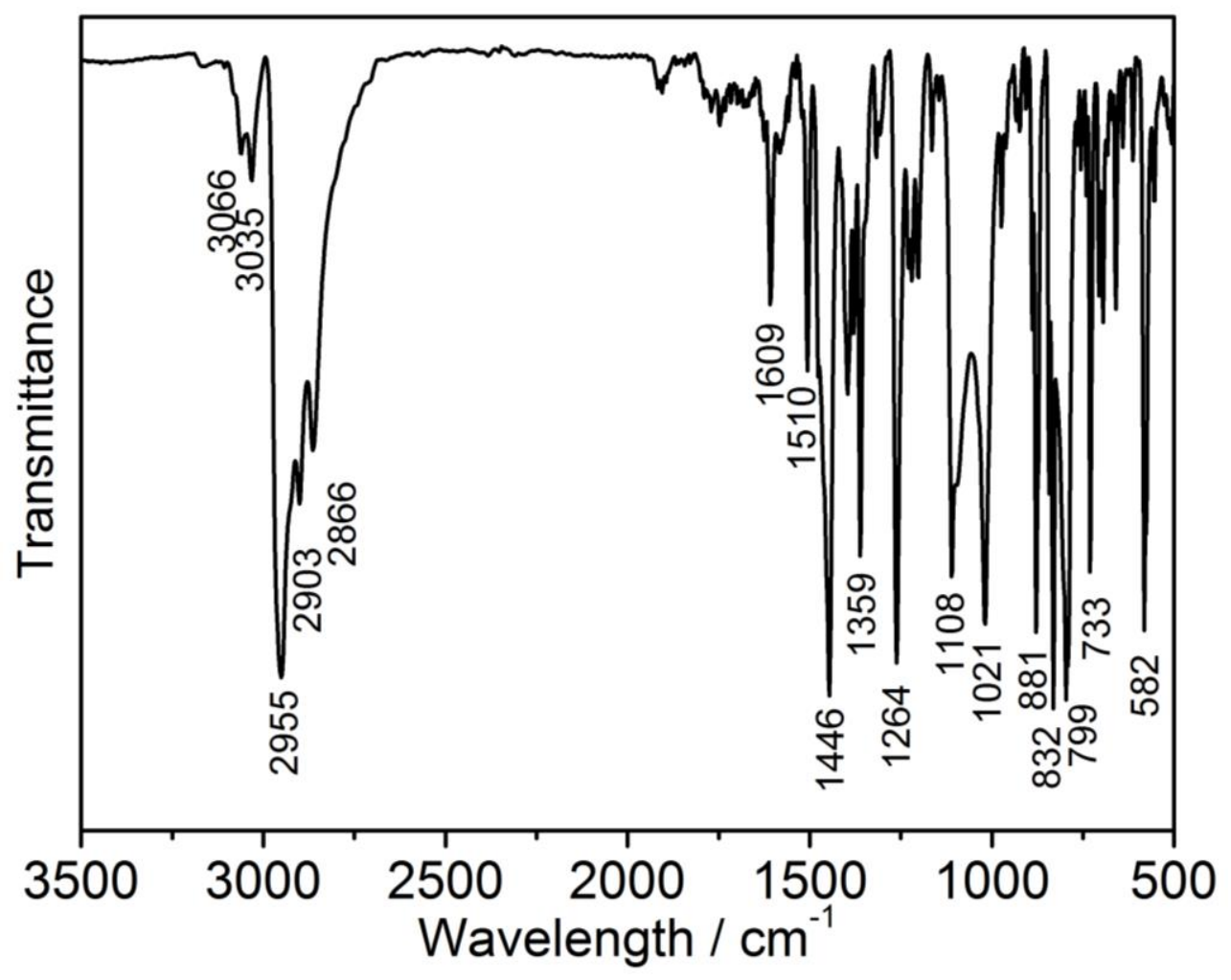

Figure S33 FT-IR spectrum of $\mathbf{9 b}$. 


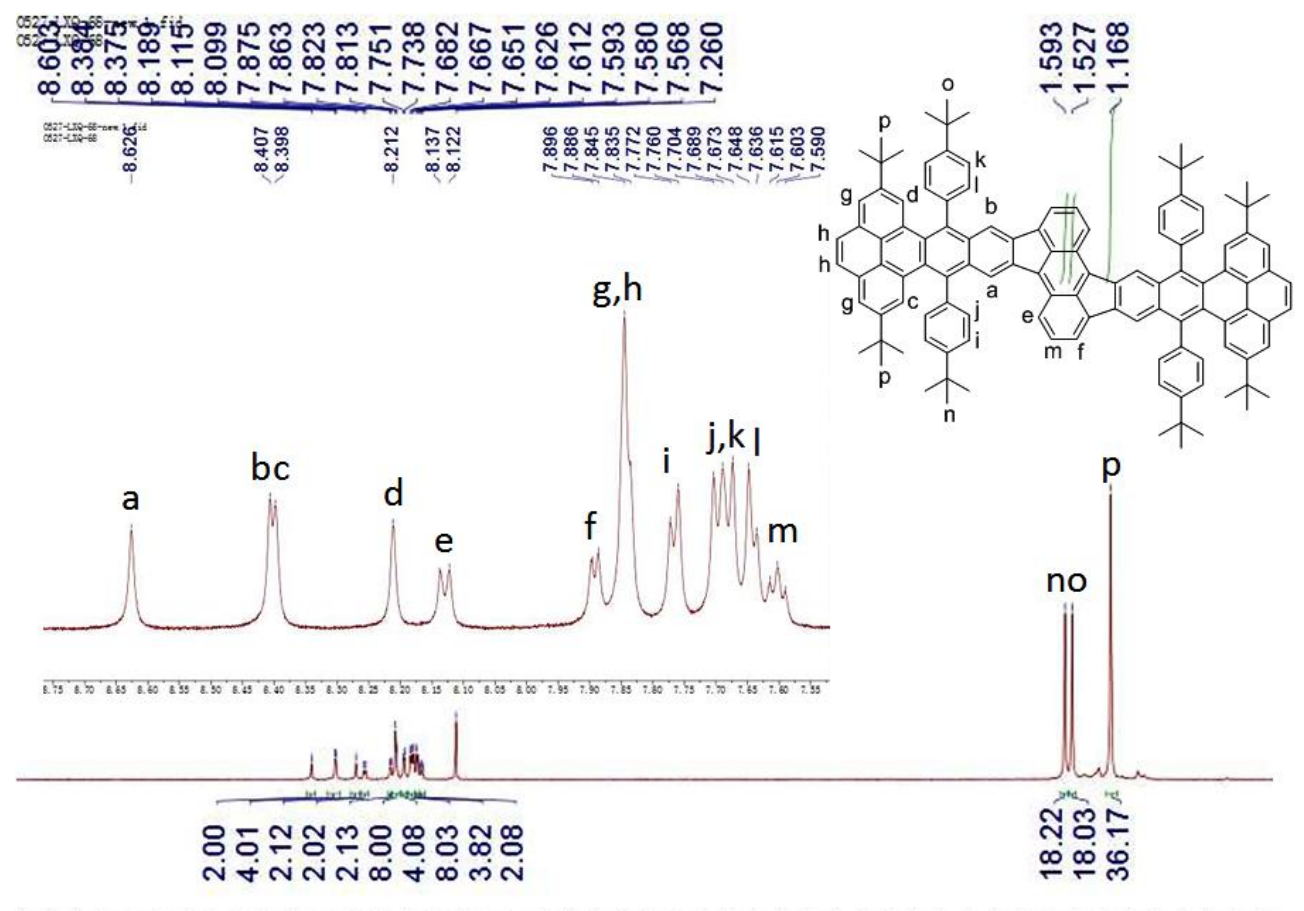

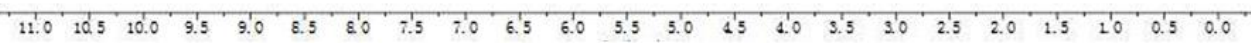

Figure $\mathrm{S} 34{ }^{1} \mathrm{H}$ NMR spectrum of $9 \mathrm{~b}$ in $\mathrm{CDCl}_{3}+\mathrm{CS}_{2}(600 \mathrm{MHz})$.

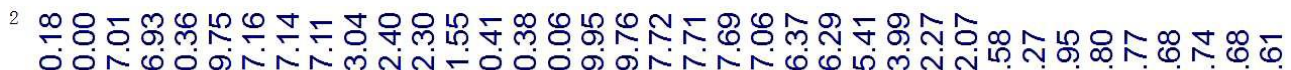

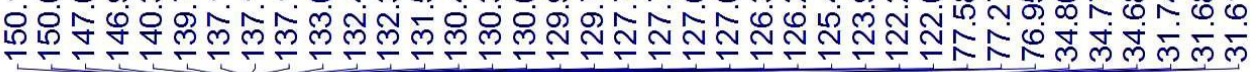

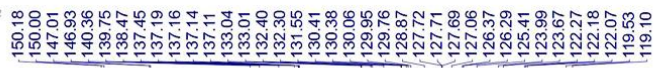

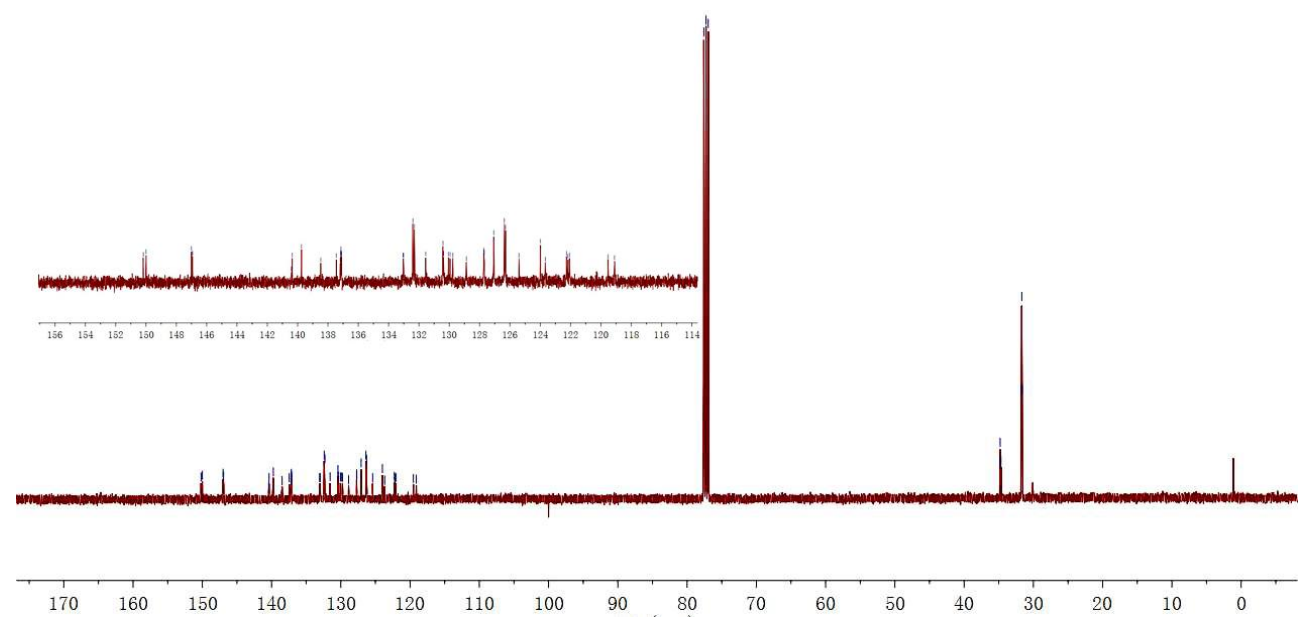

Figure $\mathrm{S} 35{ }^{13} \mathrm{C}$ NMR spectrum of $\mathbf{9 b}$ in $\mathrm{CDCl}_{3}+\mathrm{CS}_{2}(100 \mathrm{MHz})$. 


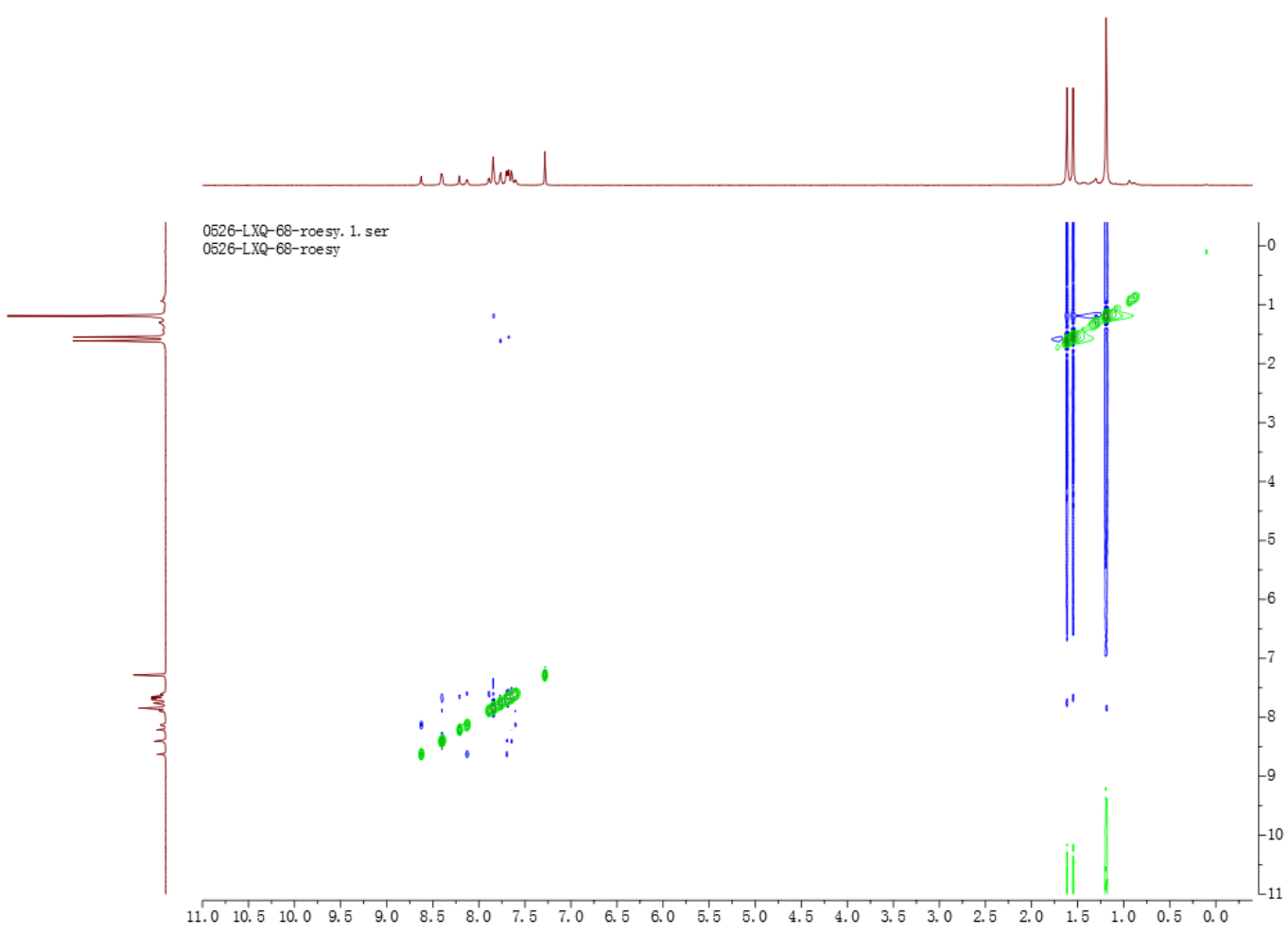

Figure 36 ROSY spectrum of $\mathbf{9 b}$ in $\mathrm{CDCl}_{3}+\mathrm{CS}_{2}(600 \mathrm{MHz})$.

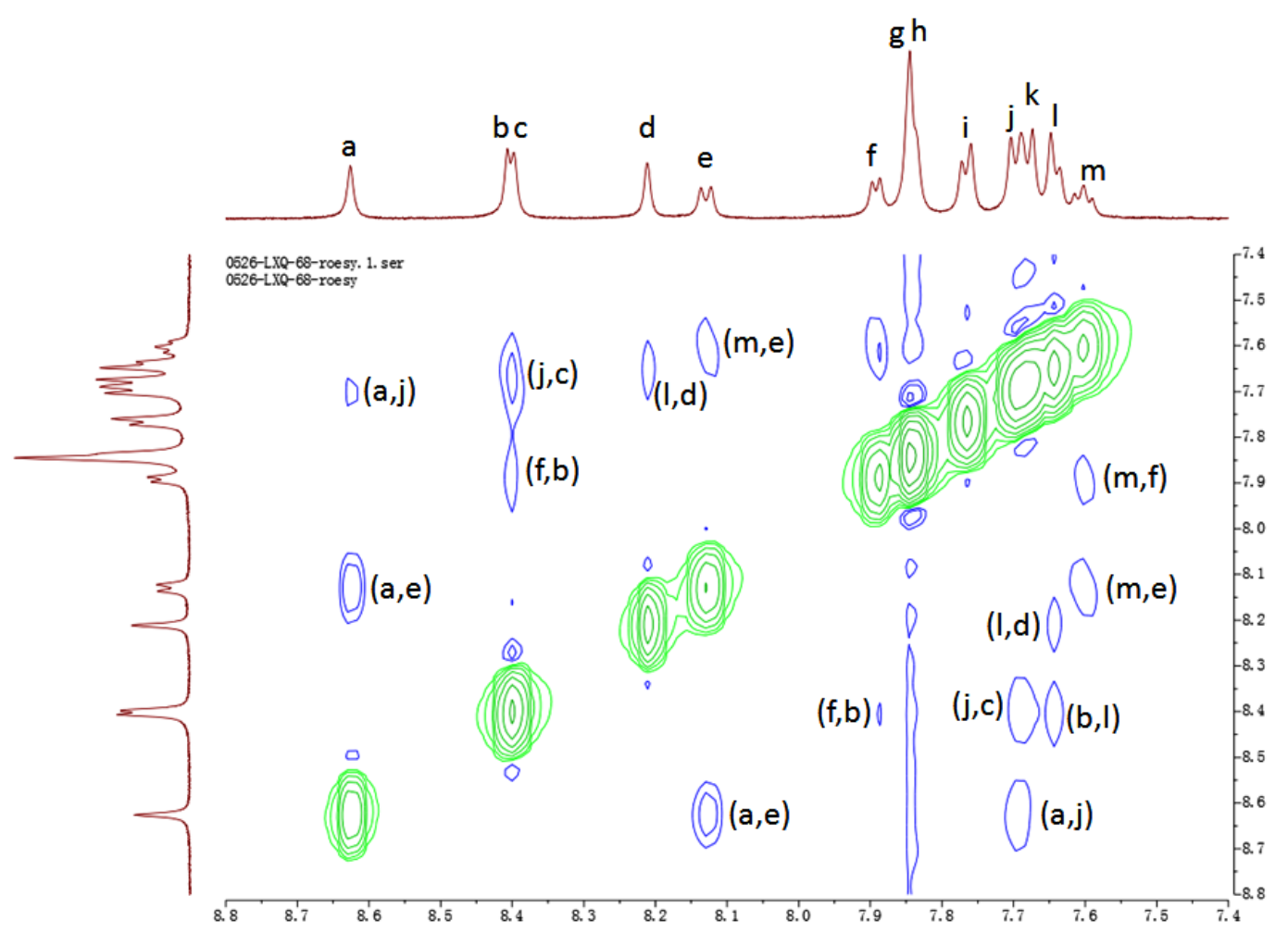

Figure 37 Magnified ROSY spectrum of $\mathbf{9 b}(7.4-8.8 \mathrm{ppm})$ in $\mathrm{CDCl}_{3}+\mathrm{CS}_{2}(600 \mathrm{MHz})$. 


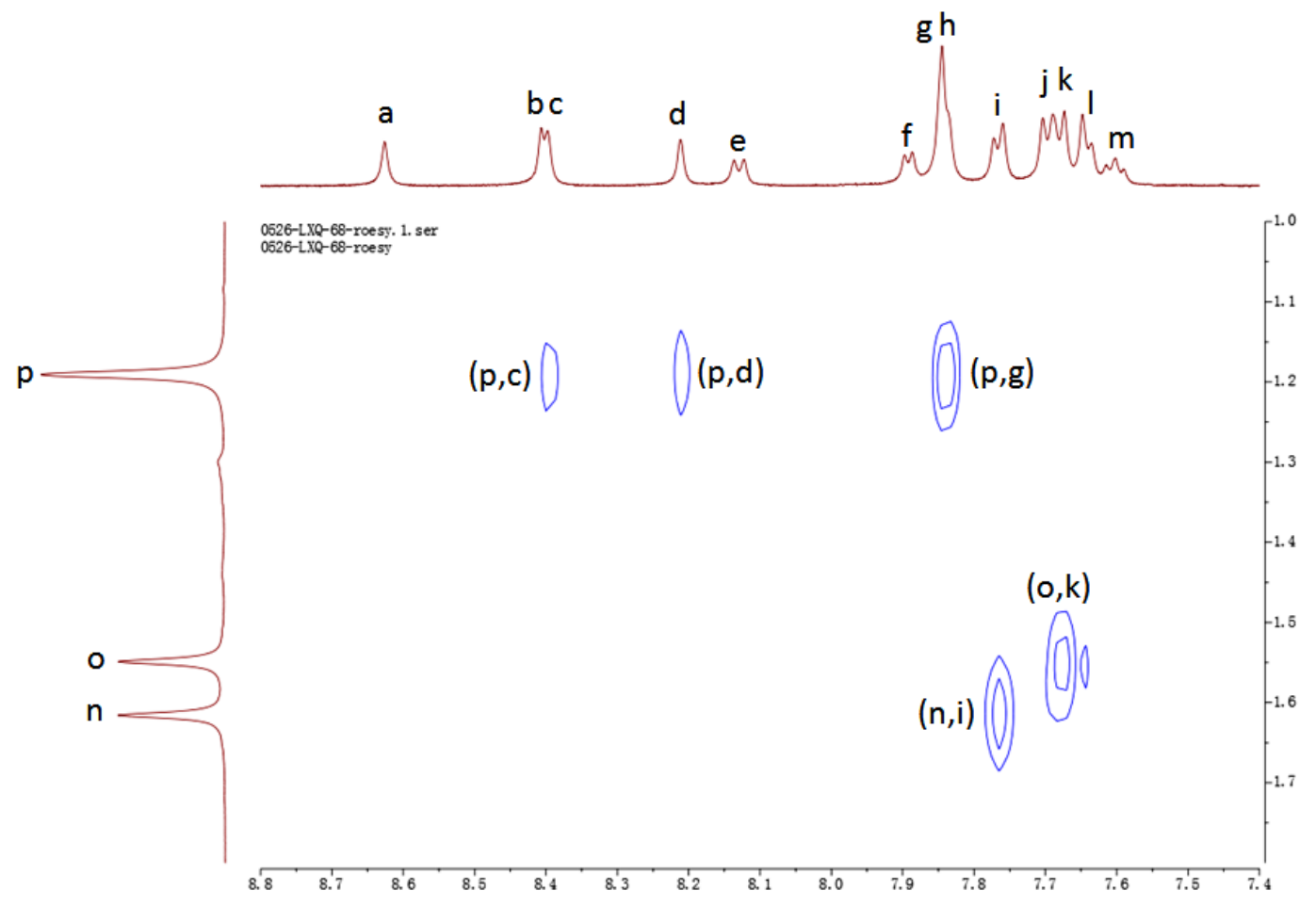

Figure 38 Magnified ROSY spectrum of $9 \mathbf{b}(8.8-7.4 / 1.0-1.8 \mathrm{ppm})$ in $\mathrm{CDCl}_{3}+\mathrm{CS}_{2}(600$ $\mathrm{MHz}$ ).

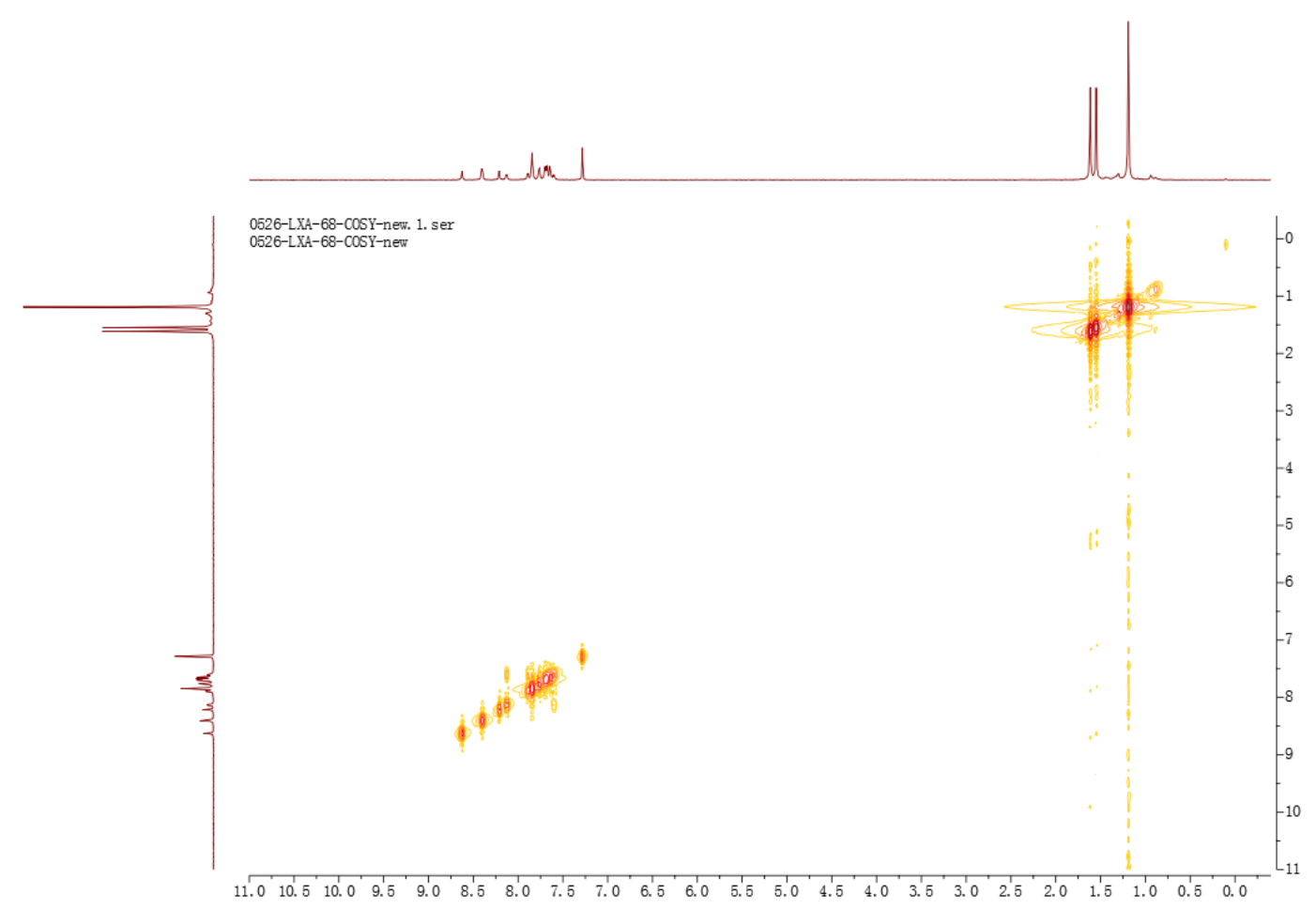

Figure $39 \mathrm{HHCOSY}$ spectrum of $\mathbf{9 b}$ in $\mathrm{CDCl}_{3}+\mathrm{CS}_{2}(600 \mathrm{MHz})$. 


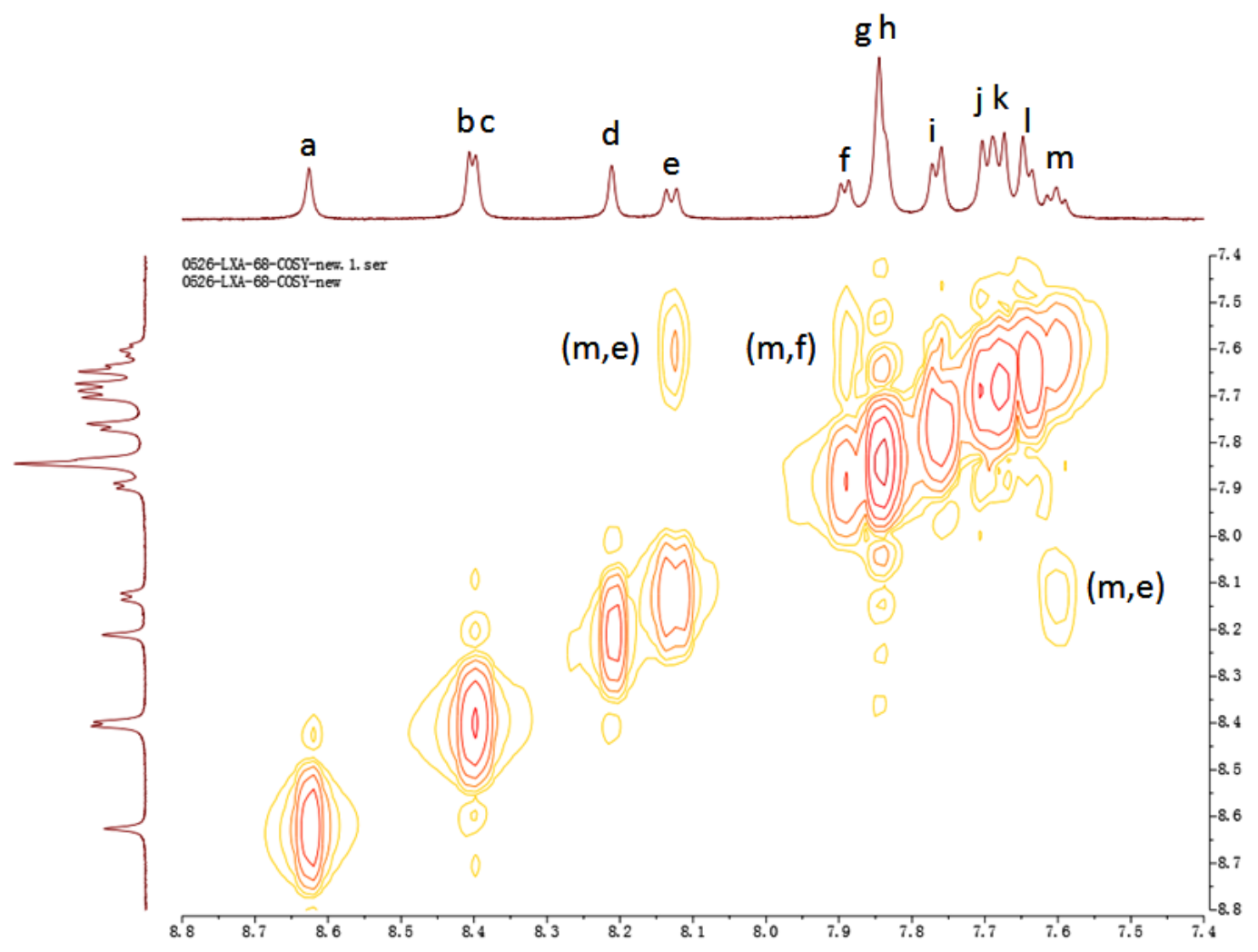

Figure 40 Magnified HHCOSY spectrum of $\mathbf{9 b}$ in $\mathrm{CDCl}_{3}+\mathrm{CS}_{2}(600 \mathrm{MHz})$.

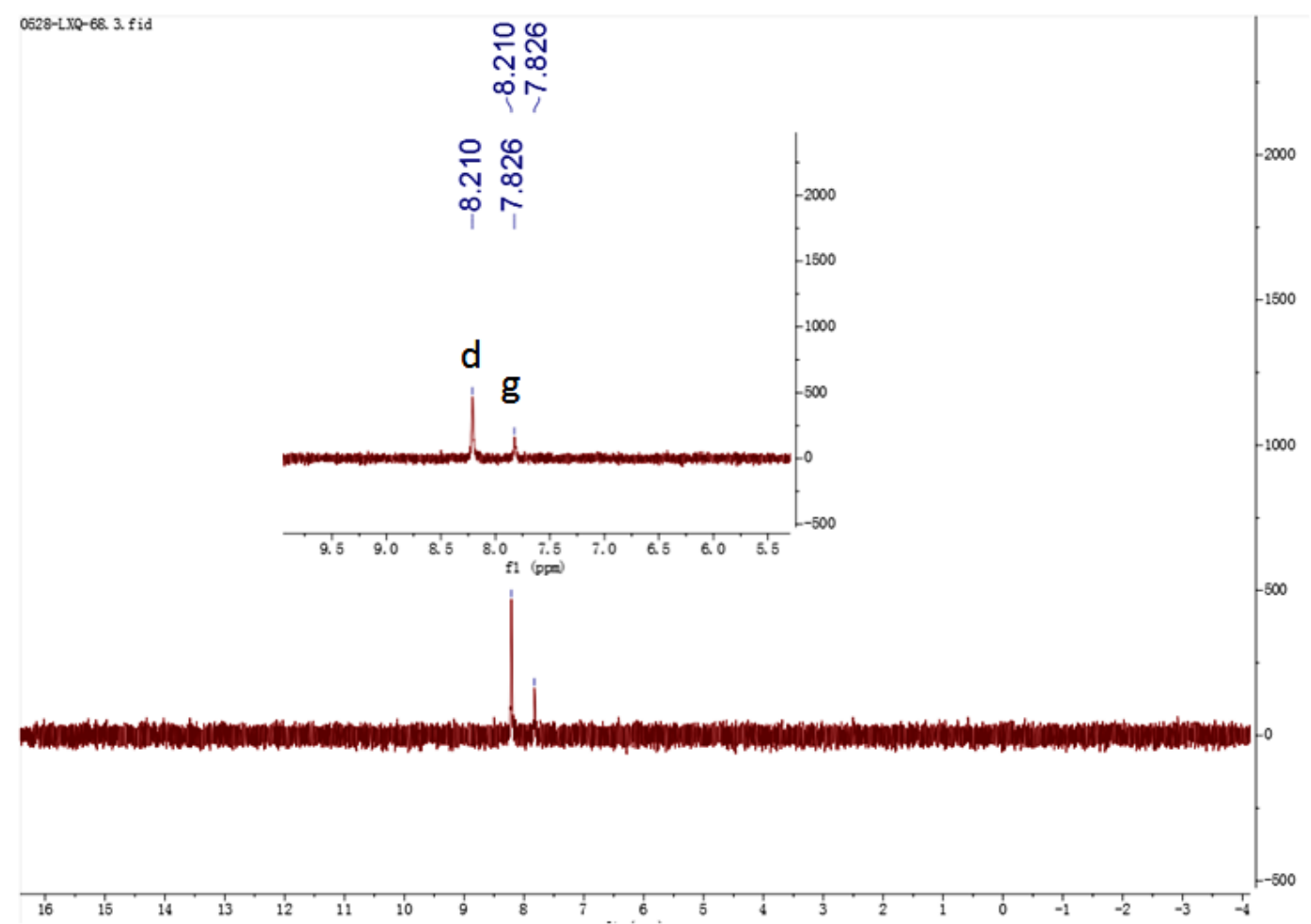

Figure 41 1D TOCSY spectrum of $\mathbf{9 b}$ in $\mathrm{CDCl}_{3}+\mathrm{CS}_{2}(600 \mathrm{MHz})$. 


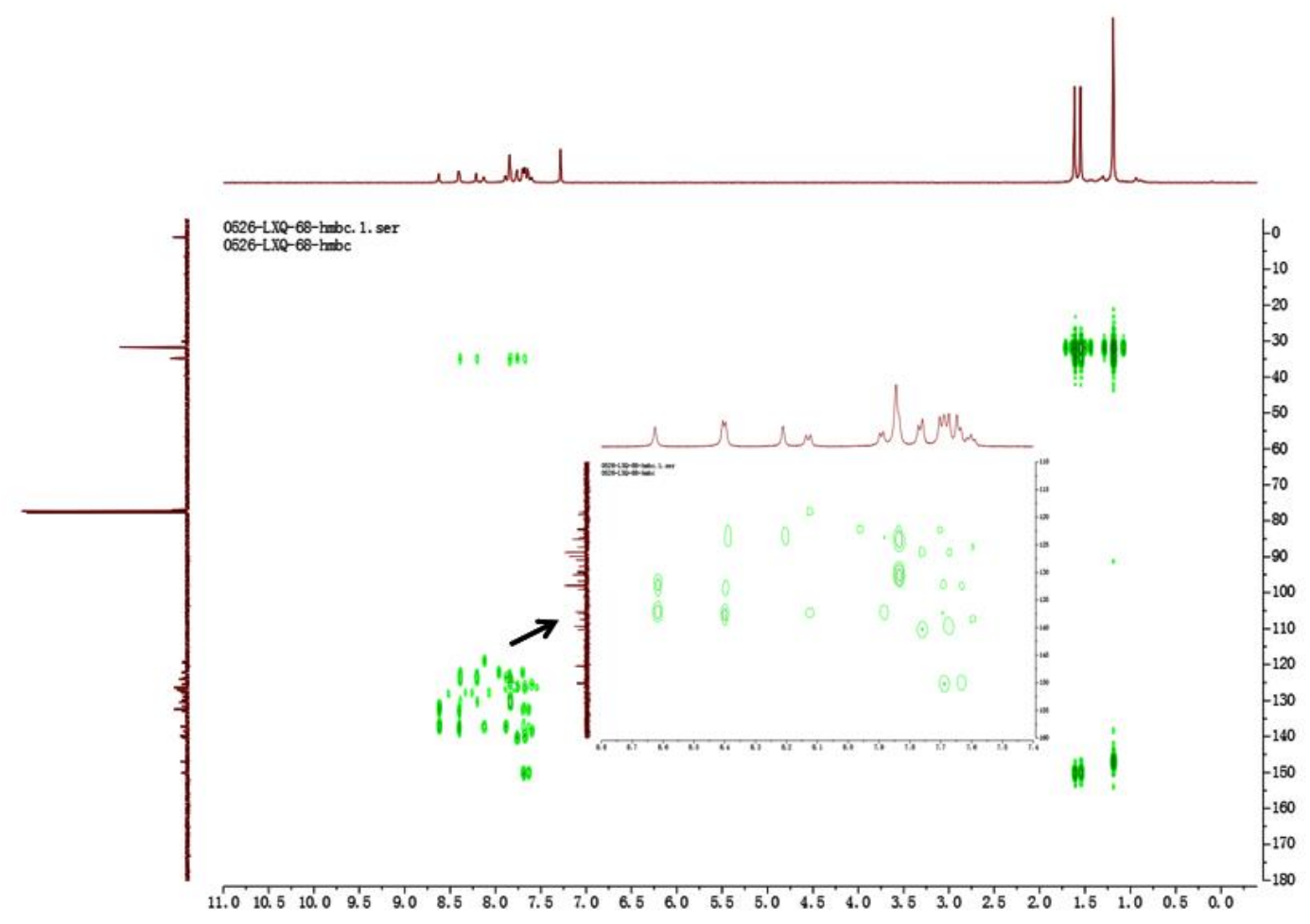

Figure $42 \mathrm{HMBC}$ spectrum of $\mathbf{9 b}$ in $\mathrm{CDCl}_{3}+\mathrm{CS}_{2}(600 \mathrm{MHz})$.

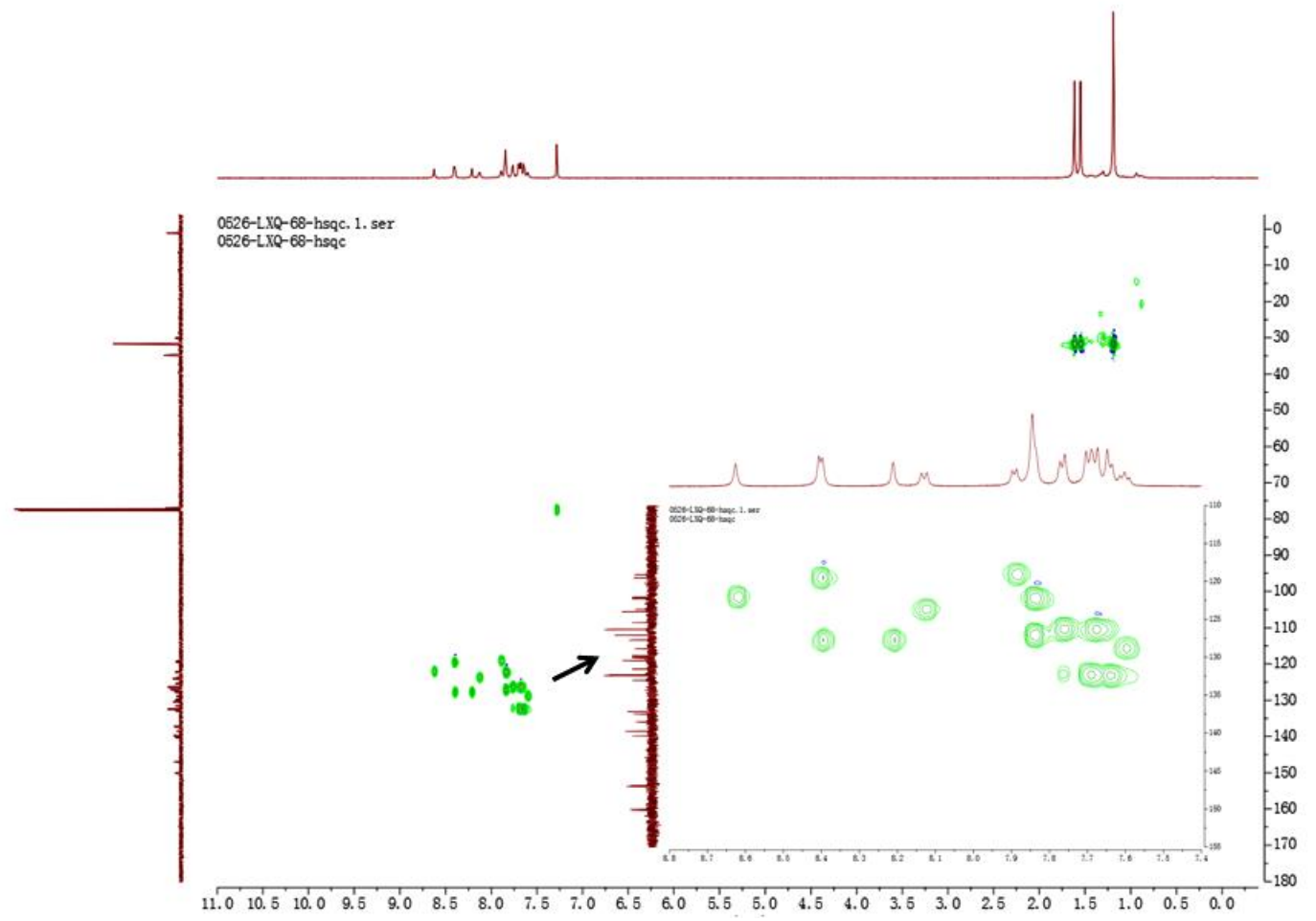

Figure $43 \mathrm{HSQC}$ spectrum of $\mathbf{9 b}$ in $\mathrm{CDCl}_{3}+\mathrm{CS}_{2}(600 \mathrm{MHz})$. 


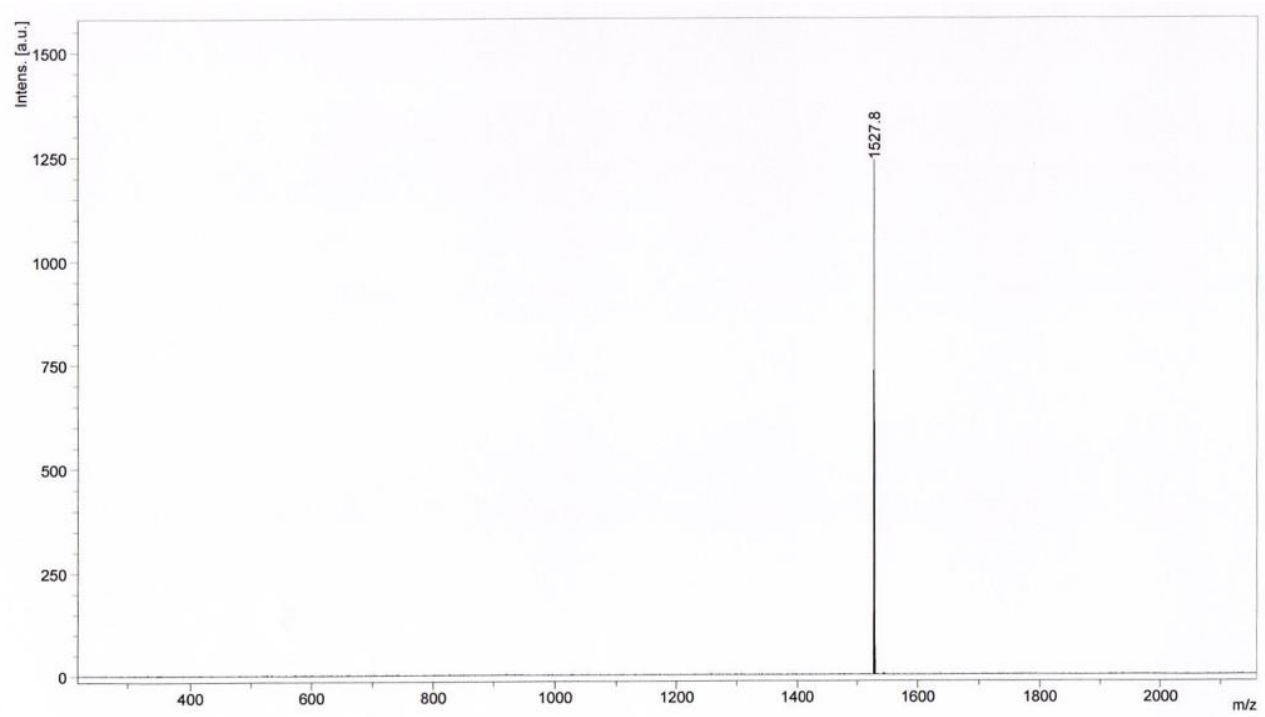

Figure S44 MALDI-TOF spectrum of $\mathbf{9 b}$.

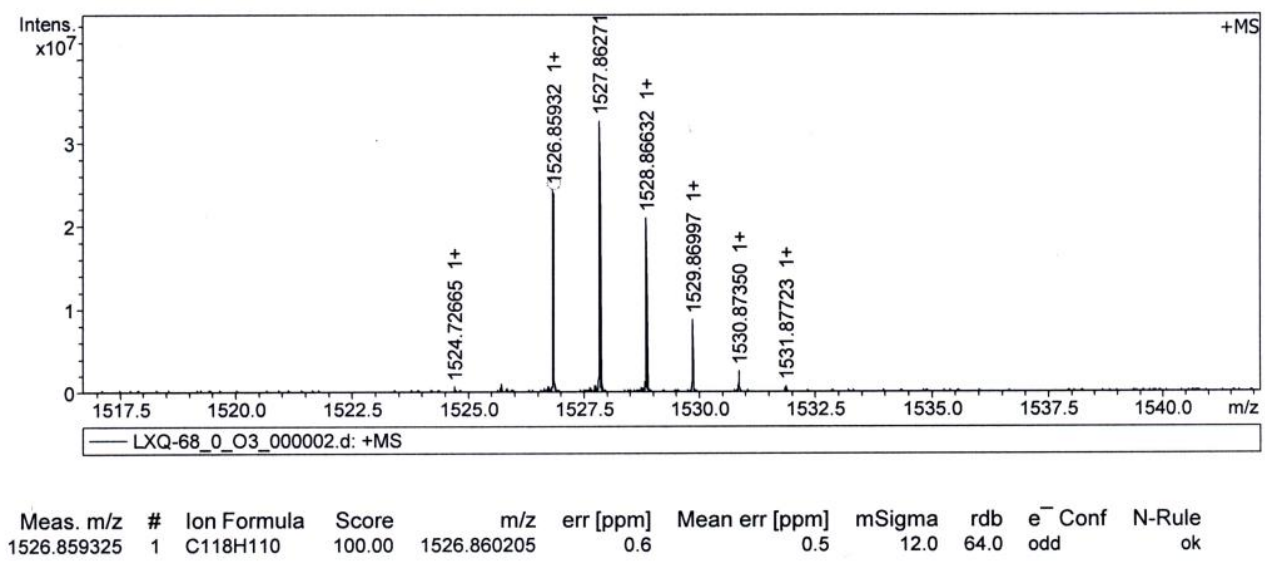

Figure S45 HR-MS spectrum of $\mathbf{9 b}$. 


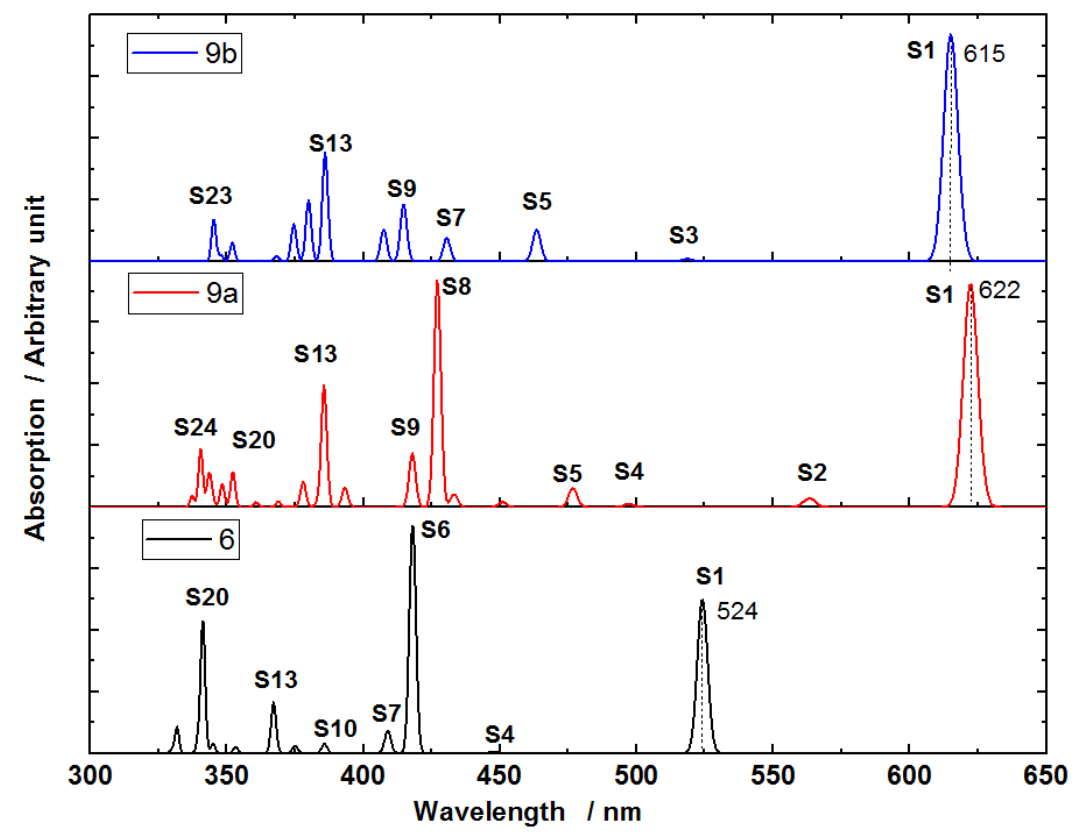

Figure S46 TDDFT-calculated absorption spectra for the low-lying 25 electronic states of neutral 6, 9a and $9 \mathbf{b}$ at B3LYP/6-31G* level.

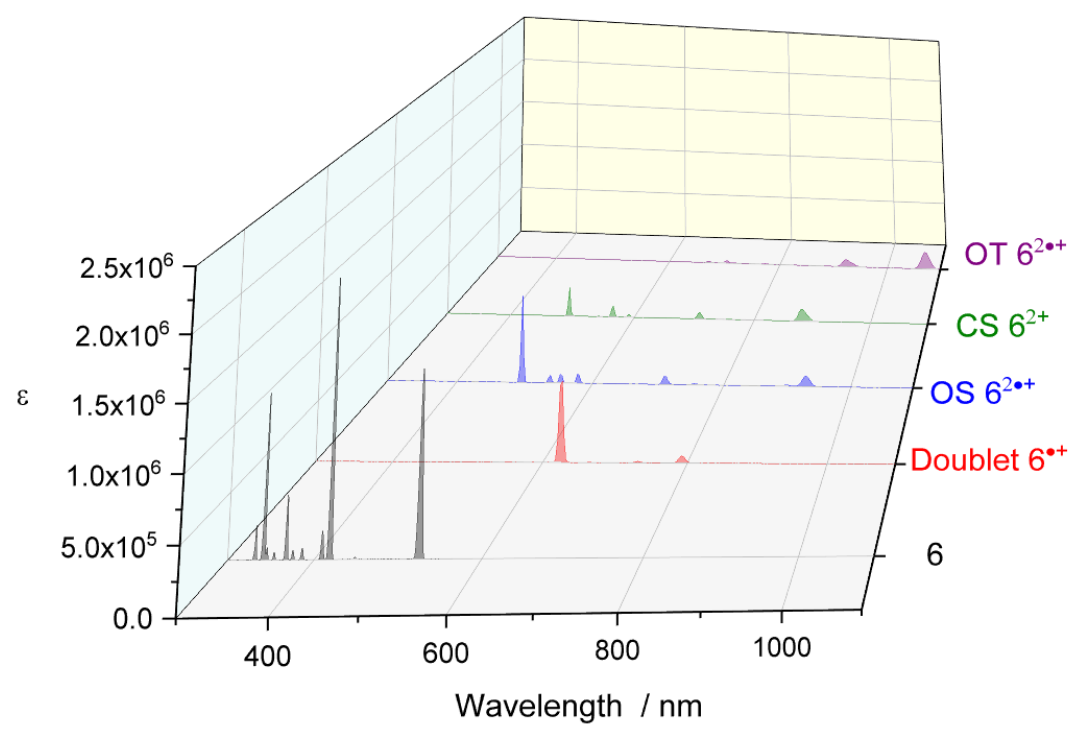

Figure S47 TDDFT-calculated absorption spectra for the low-lying 25 electronic states of $\mathbf{6}^{6^{++}}$, OS $6^{2 \cdot+}$, CS $6^{2+}$ and OT $6^{2 \cdot+}$ at B3LYP/6-31G* level. 


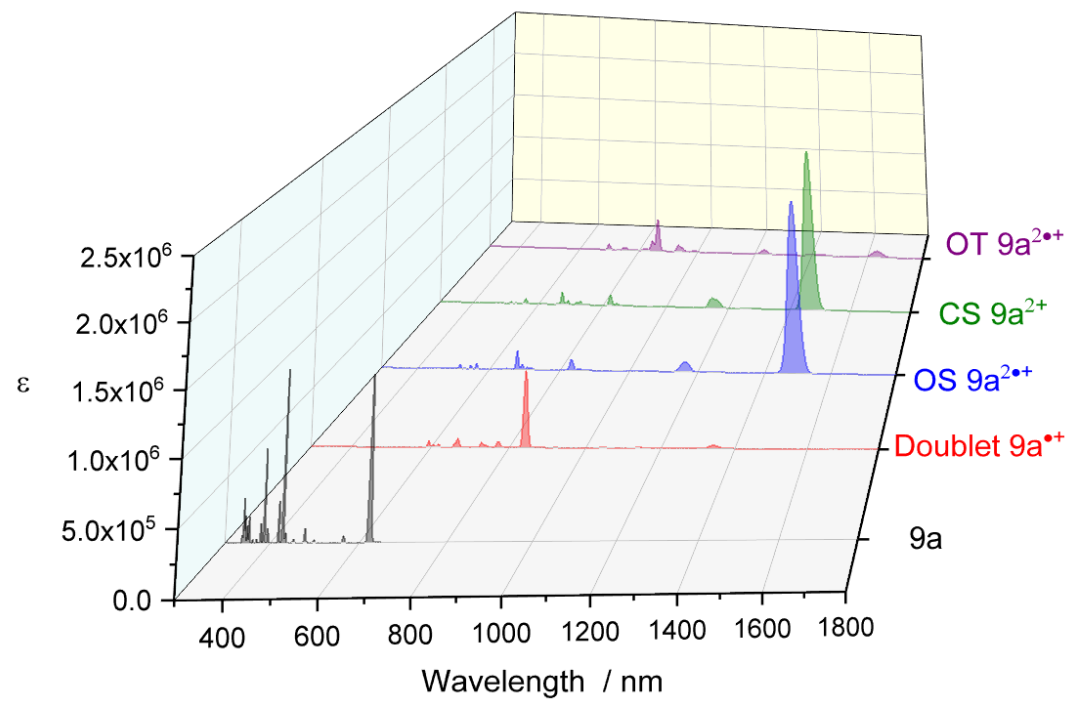

Figure S48 TDDFT-calculated absorption spectra for the low-lying 25 electronic

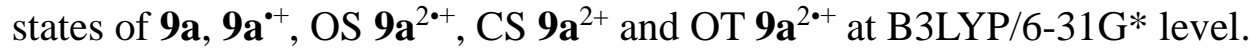

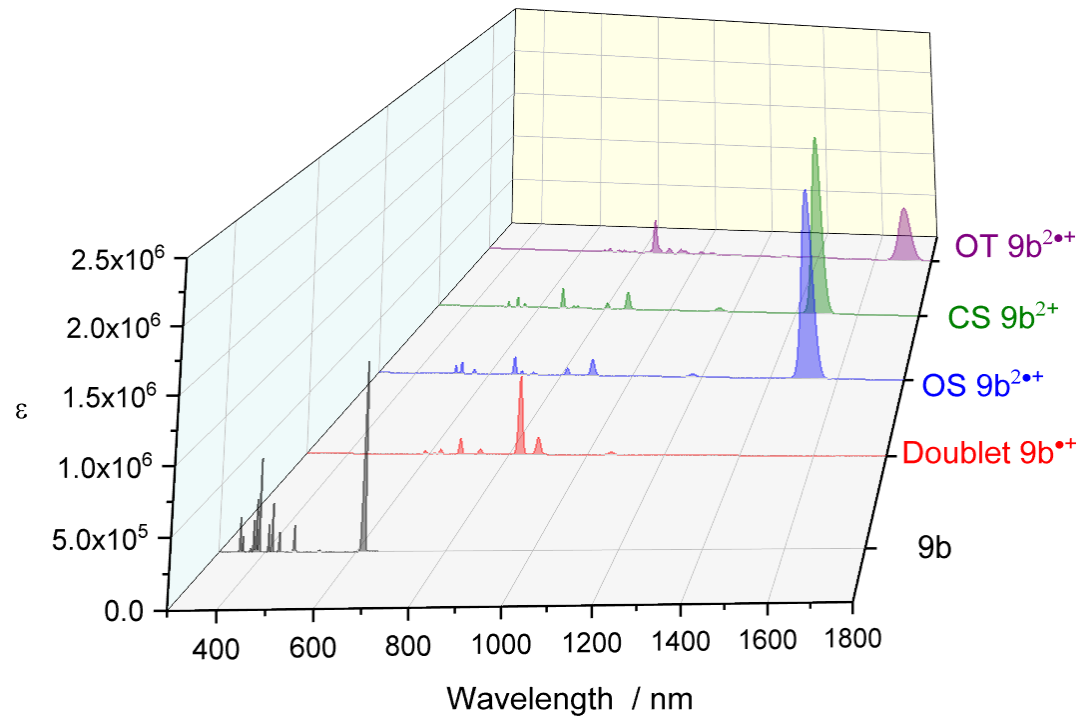

Figure S49 TDDFT-calculated absorption spectra for the low-lying 25 electronic states of $\mathbf{9 b}, \mathbf{9} \mathbf{b}^{\bullet+}$, OS $9 \mathbf{b}^{2 \cdot+}$, CS $9 \mathbf{b}^{2+}$ and OT $9 \mathbf{b}^{2 \cdot+}$ at B3LYP/6-31G* level. 

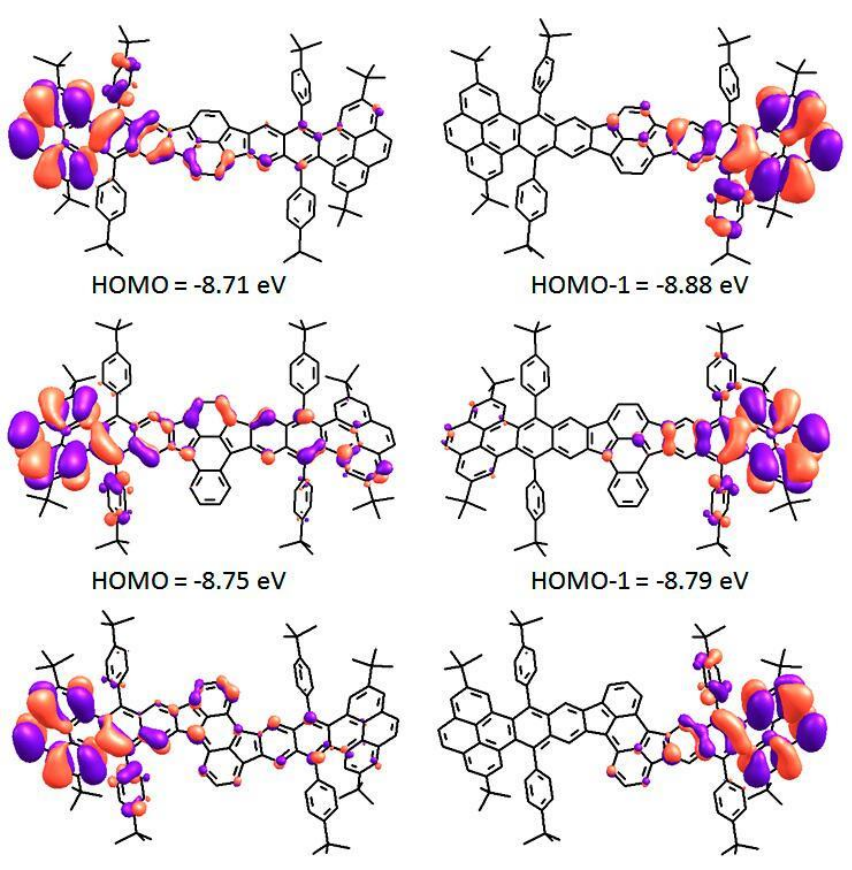

$\mathrm{HOMO}=-8.75 \mathrm{eV}$

HOMO- $1=-8.83 \mathrm{eV}$

Figure S50 Localized natural orbitals (LNOs, with isosurface $=0.0004$ ) of $\mathbf{6}^{2 \cdot+}$ (top), $\mathbf{9} \mathbf{a}^{2 \cdot+}$ (middle), and $\mathbf{9} \mathbf{b}^{2 \cdot+}$ (bottom).
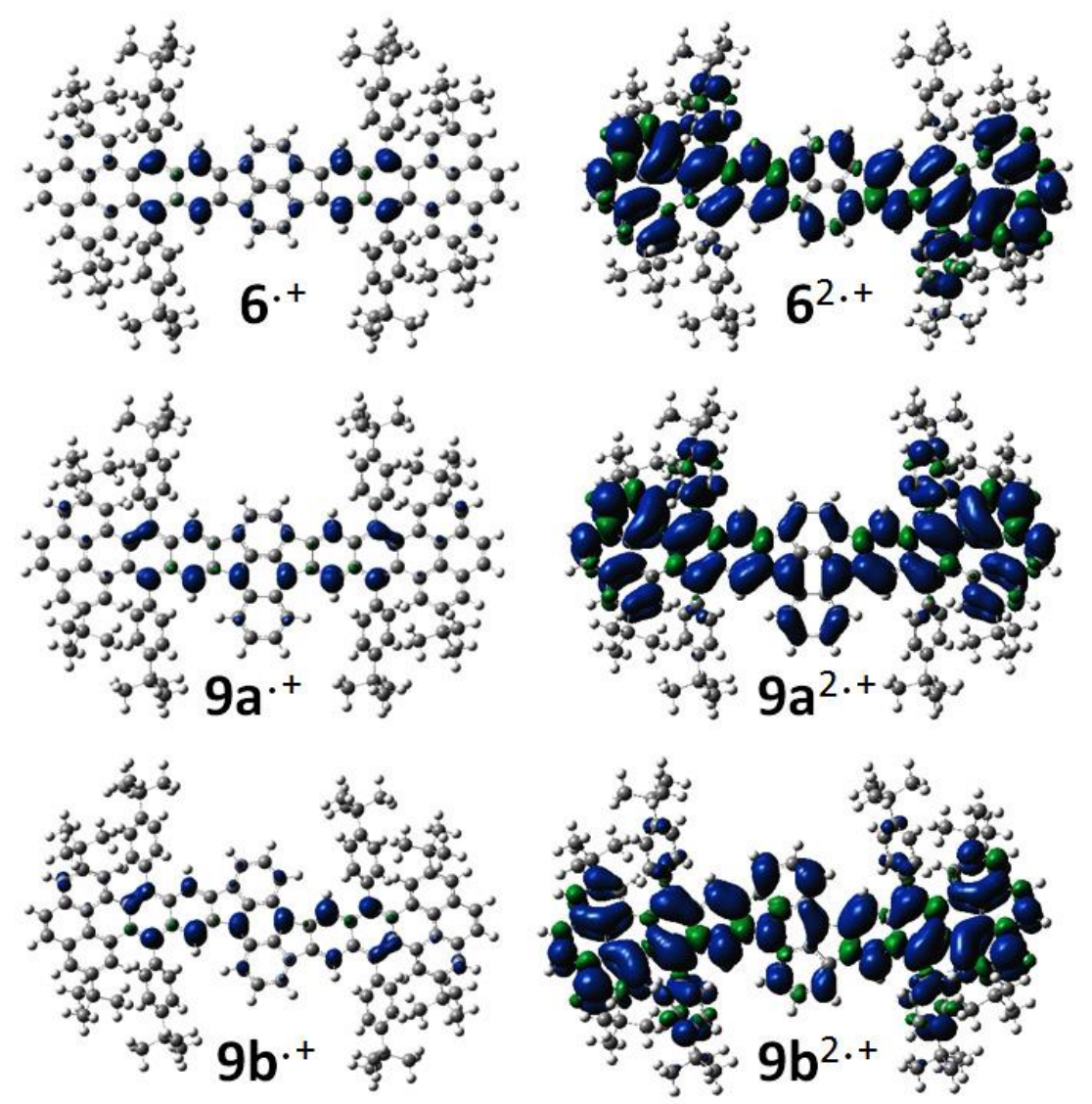

Figure S51 The spin density distributions of the cationic and dicationic radicals of $\mathbf{6}$, 9a and $9 b$. 
Table S1 Time-Dependent Density Functional Theory calculated values for the 10 lowest-lying excited singlet states of $\mathbf{6}$.

\begin{tabular}{lllll}
\hline State & $\begin{array}{l}\text { Excitation energy } \\
(\mathrm{EE})(\mathrm{eV})\end{array}$ & $\begin{array}{l}\text { Absorption } \\
\text { peak }(\mathrm{nm})\end{array}$ & $\begin{array}{l}\text { Oscillator } \\
\text { strengths } \\
(\mathrm{OS})\end{array}$ & $\begin{array}{l}\text { Dominant orbital transitions } \\
\text { contributions (DOTC) }\end{array}$ \\
\hline S1 & 2.37 & 524 & $\mathrm{f}=1.0752$ & $95 \% \mathrm{H}->\mathrm{L}$ \\
S2 & 2.53 & 491 & $\mathrm{f}=0.0000$ & $89 \% \mathrm{H}-1->\mathrm{L}$ \\
S3 & 2.74 & 452 & $\mathrm{f}=0.0000$ & $80 \% \mathrm{H}-3->\mathrm{L}$ \\
S4 & 2.77 & 448 & $\mathrm{f}=0.0131$ & $94 \% \mathrm{H}-2->\mathrm{L}$ \\
S5 & 2.81 & 441 & $\mathrm{f}=0.0000$ & $95 \% \mathrm{H}->\mathrm{L}+1$ \\
S6 & 2.97 & 418 & $\mathrm{f}=1.6045$ & $80 \% \mathrm{H}-1->\mathrm{L}+1$ \\
S7 & 3.03 & 409 & $\mathrm{f}=0.1583$ & $85 \% \mathrm{H}->\mathrm{L}+2$ \\
S8 & 3.08 & 402 & $\mathrm{f}=0.0000$ & $41 \% \mathrm{H}-2->\mathrm{L}+2,24 \% \mathrm{H}->\mathrm{L}+3$ \\
S9 & 3.12 & 398 & $\mathrm{f}=0.0000$ & $88 \% \mathrm{H}-4->\mathrm{L}$ \\
S10 & 3.21 & 386 & $\mathrm{f}=0.0658$ & $86 \% \mathrm{H}-3->\mathrm{L}+2$ \\
\hline
\end{tabular}

Table S2 Time-Dependent Density Functional Theory calculated values for the 10 lowest-lying excited singlet states of $\mathbf{9 a}$.

\begin{tabular}{lllll}
\hline State & $\begin{array}{l}\text { Excitation } \\
\text { energy } \\
(\mathrm{EE})(\mathrm{eV})\end{array}$ & $\begin{array}{l}\text { Absorption } \\
\text { peak }(\mathrm{nm})\end{array}$ & $\begin{array}{l}\text { Oscillator } \\
\text { strengths } \\
(\mathrm{OS})\end{array}$ & $\begin{array}{l}\text { Dominant orbital } \\
\text { transitions } \\
\text { contributions } \\
\text { (DOTC) }\end{array}$ \\
\hline S1 & 1.99 & 622 & $\mathrm{f}=0.9684$ & $97 \% \mathrm{H}->\mathrm{L}$ \\
S2 & 2.20 & 563 & $\mathrm{f}=0.0382$ & $91 \% \mathrm{H}-1-\mathrm{L}$ \\
S3 & 2.48 & 500 & $\mathrm{f}=0.0005$ & $83 \% \mathrm{H}-3->\mathrm{L}$ \\
S4 & 2.49 & 497 & $\mathrm{f}=0.0144$ & $87 \% \mathrm{H}-2->\mathrm{L}$ \\
S5 & 2.60 & 477 & $\mathrm{f}=0.0827$ & $96 \% \mathrm{H}->\mathrm{L}+1$ \\
S6 & 2.75 & 451 & $\mathrm{f}=0.0225$ & $83 \% \mathrm{H}-4->\mathrm{L}$ \\
S7 & 2.86 & 433 & $\mathrm{f}=0.0568$ & $94 \% \mathrm{H}->\mathrm{L}+2$ \\
S8 & 2.90 & 427 & $\mathrm{f}=0.9857$ & $60 \%(\mathrm{H}-1->\mathrm{L}+1)+23 \%(\mathrm{H}-5->\mathrm{L})$ \\
S9 & 2.97 & 418 & $\mathrm{f}=0.2350$ & $62 \%(\mathrm{H}-5->\mathrm{L})+20 \%(\mathrm{H}-1->\mathrm{L}+1)$ \\
S10 & 3.00 & 414 & $\mathrm{f}=0.0012$ & $39 \%(\mathrm{H}->\mathrm{L}+3)+33 \%(\mathrm{H}-2->\mathrm{L}+1)$ \\
\hline
\end{tabular}


Table S3 Time-Dependent Density Functional Theory calculated values for the 10 lowest-lying excited singlet states of $\mathbf{9 b}$.

$\begin{array}{lllll}\text { State } & \begin{array}{l}\text { Excitation } \\ \text { energy }\end{array} & \text { Absorption } & \begin{array}{l}\text { Oscillator } \\ \text { strengths }\end{array} & \begin{array}{l}\text { Dominant orbital transitions contributions } \\ (\mathrm{EE})(\mathrm{eV})\end{array} \\ & \text { peak }(\mathrm{nm}) & (\mathrm{OS}) & (\text { DOTC) }\end{array}$

\begin{tabular}{lllll}
\hline S1 & 2.02 & 615 & $\mathrm{f}=1.0661$ & $98 \%(\mathrm{H}->\mathrm{L})$ \\
S2 & 2.35 & 527 & $\mathrm{f}=0.0000$ & $95 \%(\mathrm{H}-1->\mathrm{L})$ \\
S3 & 2.39 & 519 & $\mathrm{f}=0.0119$ & $93 \%(\mathrm{H}-2->\mathrm{L})$ \\
S4 & 2.51 & 493 & $\mathrm{f}=0.0000$ & $96 \%(\mathrm{H}-3->\mathrm{L})$ \\
S5 & 2.68 & 463 & $\mathrm{f}=0.1494$ & $95 \%(\mathrm{H}-\mathrm{L}+1)$ \\
S6 & 2.73 & 454 & $\mathrm{f}=0.0000$ & $95 \%(\mathrm{H}-\mathrm{L}+2)$ \\
S7 & 2.88 & 431 & $\mathrm{f}=0.1107$ & $84 \%(\mathrm{H}-4->\mathrm{L})$ \\
S8 & 2.98 & 416 & $\mathrm{f}=0.0002$ & $38 \%(\mathrm{H}->\mathrm{L}+3)+25 \%(\mathrm{H}-1->\mathrm{L}+1)$ \\
S9 & 2.99 & 415 & $\mathrm{f}=0.2696$ & $47 \%(\mathrm{H}-6->\mathrm{L})+22 \%(\mathrm{H}-2->\mathrm{L}+1)$ \\
S10 & 3.04 & 407 & $\mathrm{f}=0.1505$ & $32 \%(\mathrm{H}-6->\mathrm{L})+23 \%(\mathrm{H}-2->\mathrm{L}+1)$ \\
\hline
\end{tabular}


Table S4 Magnetic Properties for the cationic and dicationic radicals of 6, 9a and 9b at ub3lyp/EPR-II level as implemented in ORCA program

$6^{+}$

!PROPERTY!

name: EPRNMR_GTensor

comment: Electronic g tensor

geom. index: 1

prop. index: 1

Source density: $1 \mathrm{SCF}$

Spin multiplicity: 2

$\mathrm{g}$ tensor:

\begin{tabular}{|c|c|c|c|}
\hline & 0 & 1 & 2 \\
\hline 0 & 2.002640 & 0.000045 & -0.000017 \\
\hline 1 & 0.000032 & 2.002681 & -0.000108 \\
\hline 2 & 0.000026 & -0.001117 & 2.002457 \\
\hline
\end{tabular}

$6^{2+\cdot}$ Triplet

!PROPERTY!

name: $\quad$ EPRNMR_GTensor

comment: Electronic g tensor

geom. index: 1

prop. index: 1

Source density: $1 \mathrm{SCF}$

Spin multiplicity: 3

g tensor:

$\begin{array}{rrrr} & 0 & 1 & 2 \\ 0 & 2.002525 & 0.000340 & -0.000188 \\ 1 & 0.000144 & 2.002736 & -0.000155 \\ 2 & 0.000362 & -0.000809 & 2.002639\end{array}$

!PROPERTY!

$9 a^{+}$

$\begin{array}{ll}\text { name: } & \text { EPRNMR_GTensor } \\ \text { comment: } & \text { Electronic g tensor }\end{array}$

geom. index: 1

prop. index: 1

Source density: $1 \mathrm{SCF}$

Spin multiplicity: 2

$\mathrm{g}$ tensor:

$\begin{array}{lll}0 & 1 & 2\end{array}$

$\begin{array}{llll}0 & 2.002475 & -0.000002 & -0.000001\end{array}$

$\begin{array}{lllll}1 & -0.000001 & 2.002743 & -0.000064\end{array}$

$\begin{array}{llll}2 & 0.000003 & -0.000843 & 2.002417\end{array}$

!PROPERTY! 
$9 \mathrm{a}^{2+\cdot}$ Triplet

!PROPERTY!

name: $\quad$ EPRNMR_GTensor

comment: Electronic g tensor

geom. index: 1

prop. index: 1

Source density: $1 \mathrm{SCF}$

Spin multiplicity: 3

g tensor:

$\begin{array}{rrrr} & 0 & 1 & 2 \\ 0 & 2.002513 & -0.000002 & -0.000000 \\ 1 & -0.000001 & 2.002716 & -0.000113 \\ 2 & -0.000007 & -0.000805 & 2.002729\end{array}$

!PROPERTY!

$9 b^{+}$

!PROPERTY!

name: EPRNMR_GTensor

comment: Electronic g tensor

geom. index: 1

prop. index: 1

Source density: $1 \mathrm{SCF}$

Spin multiplicity: 2

g tensor:

$\begin{array}{lll}0 & 1 & 2\end{array}$

$\begin{array}{llll}0 & 2.002364 & 0.000193 & 0.000026\end{array}$

$\begin{array}{llll}1 & 0.000343 & 2.002819 & 0.000046\end{array}$

$\begin{array}{llll}2 & -0.000568 & 0.000634 & 2.002436\end{array}$

!PROPERTY!

$9 b^{2+\cdot}$ Triplet

!PROPERTY!

name: $\quad$ EPRNMR_GTensor

comment: Electronic g tensor

geom. index: 1

prop. index: 1

Source density: $1 \mathrm{SCF}$

Spin multiplicity: 3

g tensor:

\begin{tabular}{rrrr} 
& 0 & 1 & 2 \\
0 & 2.002888 & 0.000008 & -0.000025 \\
1 & -0.000063 & 2.003125 & -0.000018 \\
2 & 0.000269 & -0.000953 & 2.002204 \\
\hline
\end{tabular}


Table S5 Cartesian coordinates of optimized species.

6

Energy (with Zero Point Energy correction $)=-4409.412810(-4407.520186)$ Hartree Free energy $(298 \mathrm{~K})=-4407.665261$ Hartree

Zero number of imaginary frequency

01

$\mathrm{C}$

$\mathrm{C}$

$\mathrm{C}$

$\mathrm{C}$

$\mathrm{C}$

$\mathrm{C}$

$\mathrm{C}$

$\mathrm{C}$

C

C

C

C

C

C

C

C

C

C

C

C

C

C

C

C

C

C

C

C

C

C

C

C

C

C

C

C

C

C

$\begin{array}{rrc}11.44733600 & 2.58616300 & -1.25967900 \\ 11.45316700 & 1.32007700 & -0.64721900 \\ 10.22251100 & 0.70624800 & -0.28373900 \\ 9.00098300 & 1.41970000 & -0.44651900 \\ 9.05730500 & 2.66215100 & -1.08858700 \\ 10.25951300 & 3.25897600 & -1.51915500 \\ 10.22739700 & -0.61801000 & 0.25943000 \\ 9.00902200 & -1.30988600 & 0.48571400 \\ 7.73277100 & -0.66646500 & 0.09988400 \\ 7.72702800 & 0.77141600 & -0.05106100 \\ 11.46582200 & -1.25224500 & 0.56733500 \\ 11.46223100 & -2.50742500 & 1.19423200 \\ 10.27416300 & -3.14718500 & 1.54334400 \\ 9.06638100 & -2.53606200 & 1.16552000 \\ 12.68439000 & 0.63122100 & -0.37765900 \\ 12.69204200 & -0.58717900 & 0.22595500 \\ 6.52985400 & -1.37865900 & -0.07343900 \\ 5.29003700 & -0.67420100 & -0.11056500 \\ 5.28102700 & 0.74228900 & 0.14240500 \\ 6.51174500 & 1.46513700 & 0.11247100 \\ 4.04395200 & -1.34149100 & -0.34378200 \\ 2.85296700 & -0.68220100 & -0.19655800 \\ 2.84289700 & 0.71819600 & 0.21109200 \\ 4.02417200 & 1.39312800 & 0.36609200 \\ -0.72771700 & 2.27899100 & 0.68578000 \\ 0.70708400 & 2.28832700 & 0.68938200 \\ 1.43482800 & 1.15002700 & 0.34686700 \\ 0.68944600 & 0.00421900 & 0.00091600 \\ -0.67701700 & -0.00466900 & -0.00250200 \\ -1.43891000 & 1.13136100 & 0.33965000 \\ 1.45137100 & -1.13197200 & -0.34064000 \\ 0.74016400 & -2.27967800 & -0.68647700 \\ -0.69464400 & -2.28900500 & -0.69002500 \\ -1.42243600 & -1.15066000 & -0.34776500 \\ -2.84050500 & 0.68130400 & 0.19675500 \\ -2.83052300 & -0.71913500 & -0.21089500 \\ -4.01178700 & -1.39445700 & -0.36427600 \\ -5.26838100 & -0.74410400 & -0.13817100\end{array}$




\begin{tabular}{|c|c|c|c|}
\hline $\mathrm{C}$ & -5.27773800 & 0.67247500 & 0.11551200 \\
\hline $\mathrm{C}$ & -4.03143900 & 1.34024600 & 0.34597900 \\
\hline $\mathrm{C}$ & -6.49879400 & -1.46693600 & -0.10596000 \\
\hline $\mathrm{C}$ & -7.71300000 & -0.77348800 & 0.06590900 \\
\hline $\mathrm{C}$ & -7.72142500 & 0.66402200 & -0.08508600 \\
\hline $\mathrm{C}$ & -6.51790300 & 1.37675500 & 0.08188600 \\
\hline $\mathrm{C}$ & -8.98285300 & -1.42358700 & 0.46965000 \\
\hline $\mathrm{C}$ & -10.20693100 & -0.71484300 & 0.30812700 \\
\hline $\mathrm{C}$ & -10.21679300 & 0.60851900 & -0.23710300 \\
\hline $\mathrm{C}$ & -9.00091300 & 1.30440500 & -0.46604600 \\
\hline $\mathrm{C}$ & -11.45802900 & 1.23835300 & -0.54249500 \\
\hline $\mathrm{C}$ & -11.46007100 & 2.49371000 & -1.16909000 \\
\hline $\mathrm{C}$ & -10.27490600 & 3.13800900 & -1.51967000 \\
\hline $\mathrm{C}$ & -9.06414700 & 2.53097100 & -1.14460200 \\
\hline $\mathrm{C}$ & -9.03165500 & -2.66191200 & 1.12019700 \\
\hline $\mathrm{C}$ & -10.23020400 & -3.26042900 & 1.55864700 \\
\hline $\mathrm{C}$ & -11.42159200 & -2.59481500 & 1.29626900 \\
\hline $\mathrm{C}$ & -11.43424300 & -1.33193500 & 0.67696700 \\
\hline $\mathrm{C}$ & -12.66839300 & -0.64828200 & 0.40757100 \\
\hline $\mathrm{C}$ & -12.68123000 & 0.56903900 & -0.19828800 \\
\hline $\mathrm{C}$ & 6.45422900 & 2.94170600 & 0.35558700 \\
\hline $\mathrm{C}$ & 10.21691400 & 4.61943800 & -2.24038700 \\
\hline $\mathrm{C}$ & 11.60936200 & 5.06787500 & -2.72416200 \\
\hline $\mathrm{C}$ & 9.67243800 & 5.69570300 & -1.27221900 \\
\hline $\mathrm{C}$ & 9.29120200 & 4.52668200 & -3.47665900 \\
\hline $\mathrm{C}$ & 10.32166200 & -4.49048300 & 2.29816800 \\
\hline $\mathrm{C}$ & 8.92440000 & -4.98333700 & 2.71902500 \\
\hline $\mathrm{C}$ & 11.17624100 & -4.34082300 & 3.57927900 \\
\hline $\mathrm{C}$ & 10.95799200 & -5.56183500 & 1.38062900 \\
\hline $\mathrm{C}$ & 6.51253500 & -2.85478800 & -0.32197400 \\
\hline $\mathrm{C}$ & -6.49965900 & 2.85334300 & 0.32721200 \\
\hline $\mathrm{C}$ & -6.44841200 & -2.94168700 & -0.36084300 \\
\hline $\mathrm{C}$ & -10.32852900 & 4.48191400 & -2.27303300 \\
\hline $\mathrm{C}$ & -8.93386900 & 4.97977700 & -2.69662700 \\
\hline $\mathrm{C}$ & -10.96618700 & 5.55023600 & -1.35288000 \\
\hline $\mathrm{C}$ & -11.18557500 & 4.33084900 & -3.55232700 \\
\hline $\mathrm{C}$ & -10.17883200 & -4.61062500 & 2.29854000 \\
\hline $\mathrm{C}$ & -9.59869200 & -5.69319300 & 1.35862500 \\
\hline $\mathrm{C}$ & -11.57234400 & -5.07517700 & 2.76347300 \\
\hline $\mathrm{C}$ & -9.27749900 & -4.48239000 & 3.54973000 \\
\hline $\mathrm{C}$ & 5.75244400 & 3.81872000 & -0.48926500 \\
\hline $\mathrm{C}$ & 5.64326000 & 5.17415200 & -0.18912700 \\
\hline $\mathrm{C}$ & 6.22074500 & 5.72537800 & 0.96877400 \\
\hline $\mathrm{C}$ & 6.92876800 & 4.84678000 & 1.80028600 \\
\hline
\end{tabular}




\begin{tabular}{|c|c|c|c|}
\hline $\mathrm{C}$ & 7.04360900 & 3.48741600 & 1.50222500 \\
\hline $\mathrm{C}$ & 5.80968200 & -3.75214000 & 0.49327800 \\
\hline $\mathrm{C}$ & 5.77232600 & -5.11881100 & 0.20526700 \\
\hline $\mathrm{C}$ & 6.43049900 & -5.65184200 & -0.91168600 \\
\hline $\mathrm{C}$ & 7.13094300 & -4.74490900 & -1.72658900 \\
\hline $\mathrm{C}$ & 7.17227600 & -3.38352700 & -1.44308400 \\
\hline $\mathrm{C}$ & -5.74940300 & -3.83030600 & 0.46768000 \\
\hline $\mathrm{C}$ & -5.65390700 & -5.18930000 & 0.15781500 \\
\hline $\mathrm{C}$ & -6.24428900 & -5.72233600 & -0.99681900 \\
\hline $\mathrm{C}$ & -6.94943100 & -4.82592000 & -1.81881500 \\
\hline $\mathrm{C}$ & -7.05210900 & -3.47242800 & -1.51170700 \\
\hline $\mathrm{C}$ & -5.80047100 & 3.74871200 & -0.49334500 \\
\hline $\mathrm{C}$ & -5.76228300 & 5.11613500 & -0.20904800 \\
\hline $\mathrm{C}$ & -6.41585400 & 5.65187300 & 0.90931400 \\
\hline $\mathrm{C}$ & -7.11251500 & 4.74687000 & 1.72961000 \\
\hline $\mathrm{C}$ & -7.15470600 & 3.38474700 & 1.44981200 \\
\hline $\mathrm{C}$ & 6.03987800 & 7.22100200 & 1.28575900 \\
\hline $\mathrm{C}$ & 4.53315000 & 7.51989400 & 1.47607300 \\
\hline $\mathrm{C}$ & 6.58457600 & 8.07637400 & 0.11755400 \\
\hline $\mathrm{C}$ & 6.77939500 & 7.64023500 & 2.57043600 \\
\hline $\mathrm{C}$ & 6.40174800 & -7.14853500 & -1.26927600 \\
\hline $\mathrm{C}$ & 7.84719700 & -7.69854000 & -1.31528800 \\
\hline $\mathrm{C}$ & 5.74212600 & -7.33053000 & -2.65738800 \\
\hline $\mathrm{C}$ & 5.60514100 & -7.98017100 & -0.24616500 \\
\hline $\mathrm{C}$ & -6.38614300 & 7.14952800 & 1.26279100 \\
\hline $\mathrm{C}$ & -7.83160700 & 7.69910600 & 1.31336300 \\
\hline $\mathrm{C}$ & -5.59413200 & 7.97870300 & 0.23413000 \\
\hline $\mathrm{C}$ & -5.72078800 & 7.33551000 & 2.64763200 \\
\hline $\mathrm{C}$ & -6.13196700 & -7.20647800 & -1.38965800 \\
\hline $\mathrm{C}$ & -5.39455200 & -7.31922600 & -2.74574100 \\
\hline $\mathrm{C}$ & -7.54555600 & -7.82014300 & -1.52621500 \\
\hline $\mathrm{C}$ & -5.35114700 & -8.03027400 & -0.34814100 \\
\hline $\mathrm{H}$ & 12.40329900 & 3.01637700 & -1.53902600 \\
\hline $\mathrm{H}$ & 8.13411400 & 3.18581400 & -1.28498800 \\
\hline $\mathrm{H}$ & 12.42153100 & -2.96475400 & 1.42293600 \\
\hline $\mathrm{H}$ & 8.13983900 & -3.02304000 & 1.42362600 \\
\hline $\mathrm{H}$ & 13.61708400 & 1.11874100 & -0.65169400 \\
\hline $\mathrm{H}$ & 13.63060500 & -1.08885400 & 0.44973400 \\
\hline $\mathrm{H}$ & 4.05189500 & -2.38732000 & -0.62522300 \\
\hline $\mathrm{H}$ & 4.01505200 & 2.43890700 & 0.64735000 \\
\hline $\mathrm{H}$ & -1.24250000 & 3.19556300 & 0.96320000 \\
\hline $\mathrm{H}$ & 1.20852900 & 3.21151500 & 0.96928500 \\
\hline $\mathrm{H}$ & 1.25494800 & -3.19640300 & -0.96339700 \\
\hline $\mathrm{H}$ & -1.19609000 & -3.21234400 & -0.96942900 \\
\hline
\end{tabular}




\begin{tabular}{|c|c|c|c|}
\hline $\mathrm{H}$ & -4.00321100 & -2.44046500 & -0.64477200 \\
\hline $\mathrm{H}$ & -4.03922200 & 2.38603600 & 0.62763200 \\
\hline $\mathrm{H}$ & -12.42139100 & 2.94776100 & -1.39583000 \\
\hline $\mathrm{H}$ & -8.13995300 & 3.02160000 & -1.40416400 \\
\hline $\mathrm{H}$ & -8.10510100 & -3.17934500 & 1.31810900 \\
\hline $\mathrm{H}$ & -12.37509800 & -3.02747900 & 1.58019300 \\
\hline $\mathrm{H}$ & -13.59896600 & -1.13828100 & 0.68438800 \\
\hline $\mathrm{H}$ & -13.62196400 & 1.06705800 & -0.42112900 \\
\hline $\mathrm{H}$ & 12.30618200 & 5.21086200 & -1.89046800 \\
\hline $\mathrm{H}$ & 11.52552200 & 6.02642500 & -3.24871400 \\
\hline $\mathrm{H}$ & 12.05136900 & 4.34574600 & -3.42026200 \\
\hline $\mathrm{H}$ & 10.32219300 & 5.79477100 & -0.39484700 \\
\hline $\mathrm{H}$ & 9.62704700 & 6.67084000 & -1.77396300 \\
\hline $\mathrm{H}$ & 8.66675700 & 5.45201500 & -0.91743100 \\
\hline $\mathrm{H}$ & 9.65109100 & 3.76739400 & -4.18044300 \\
\hline $\mathrm{H}$ & 9.26230800 & 5.48979800 & -4.00100900 \\
\hline $\mathrm{H}$ & 8.26332700 & 4.26933100 & -3.20211200 \\
\hline $\mathrm{H}$ & 8.42052000 & -4.26247800 & 3.37343900 \\
\hline $\mathrm{H}$ & 8.27617800 & -5.17225600 & 1.85735600 \\
\hline $\mathrm{H}$ & 9.01990900 & -5.92353300 & 3.27463900 \\
\hline $\mathrm{H}$ & 10.74932700 & -3.58536100 & 4.24881600 \\
\hline $\mathrm{H}$ & 12.20655600 & -4.04519600 & 3.35658000 \\
\hline $\mathrm{H}$ & 11.21512900 & -5.29303600 & 4.12246500 \\
\hline $\mathrm{H}$ & 11.97384700 & -5.28496500 & 1.07835800 \\
\hline $\mathrm{H}$ & 11.01416900 & -6.52702600 & 1.89965800 \\
\hline $\mathrm{H}$ & 10.36327400 & -5.69757200 & 0.47009100 \\
\hline $\mathrm{H}$ & -8.42905600 & 4.26116900 & -3.35279400 \\
\hline $\mathrm{H}$ & -9.03378400 & 5.92012700 & -3.25120500 \\
\hline $\mathrm{H}$ & -8.28427900 & 5.17012300 & -1.83630500 \\
\hline $\mathrm{H}$ & -10.36970100 & 5.68705500 & -0.44366000 \\
\hline $\mathrm{H}$ & -11.98037300 & 5.26967100 & -1.04842600 \\
\hline $\mathrm{H}$ & -11.02681600 & 6.51575800 & -1.87079100 \\
\hline $\mathrm{H}$ & -10.75778900 & 3.57746000 & -4.22364200 \\
\hline $\mathrm{H}$ & -11.22874800 & 5.28350000 & -4.09441700 \\
\hline $\mathrm{H}$ & -12.21444000 & 4.03172300 & -3.32760800 \\
\hline $\mathrm{H}$ & -10.22633400 & -5.81363900 & 0.46809700 \\
\hline $\mathrm{H}$ & -9.55324300 & -6.66023700 & 1.87594600 \\
\hline $\mathrm{H}$ & -8.58796500 & -5.44295500 & 1.02328500 \\
\hline $\mathrm{H}$ & -12.03649600 & -4.35377500 & 3.44571900 \\
\hline $\mathrm{H}$ & -12.25282100 & -5.23557400 & 1.91945300 \\
\hline $\mathrm{H}$ & -11.48321300 & -6.02751900 & 3.29835100 \\
\hline $\mathrm{H}$ & -9.66577500 & -3.72170800 & 4.23671200 \\
\hline $\mathrm{H}$ & -8.25092300 & -4.20578200 & 3.28908700 \\
\hline $\mathrm{H}$ & -9.23729400 & -5.43765300 & 4.08763600 \\
\hline
\end{tabular}




\begin{tabular}{|c|c|c|c|}
\hline $\mathrm{H}$ & 5.28411300 & 3.43076400 & -1.39021200 \\
\hline $\mathrm{H}$ & 5.08689600 & 5.81082900 & -0.87187700 \\
\hline $\mathrm{H}$ & 7.39727300 & 5.21233700 & 2.70740600 \\
\hline $\mathrm{H}$ & 7.59123200 & 2.83639300 & 2.17790300 \\
\hline $\mathrm{H}$ & 5.28953100 & -3.38027000 & 1.37221100 \\
\hline $\mathrm{H}$ & 5.21628300 & -5.76749200 & 0.87334500 \\
\hline $\mathrm{H}$ & 7.65298900 & -5.10390700 & -2.60946700 \\
\hline $\mathrm{H}$ & 7.72108900 & -2.71415300 & -2.09942000 \\
\hline $\mathrm{H}$ & -5.27099800 & -3.45546100 & 1.36895100 \\
\hline $\mathrm{H}$ & -5.10111000 & -5.83109400 & 0.83517400 \\
\hline $\mathrm{H}$ & -7.42438200 & -5.18587300 & -2.72747400 \\
\hline $\mathrm{H}$ & -7.59849300 & -2.80981700 & -2.17698100 \\
\hline $\mathrm{H}$ & -5.28384300 & 3.37459600 & -1.37339800 \\
\hline $\mathrm{H}$ & -5.20927000 & 5.76324000 & -0.88115400 \\
\hline $\mathrm{H}$ & -7.63082400 & 5.10803300 & 2.61380600 \\
\hline $\mathrm{H}$ & -7.70050700 & 2.71690900 & 2.11021300 \\
\hline $\mathrm{H}$ & 3.95512600 & 7.28128300 & 0.57693900 \\
\hline $\mathrm{H}$ & 4.11906400 & 6.93482600 & 2.30523900 \\
\hline $\mathrm{H}$ & 4.37976100 & 8.58279200 & 1.69982600 \\
\hline $\mathrm{H}$ & 7.65487400 & 7.89785200 & -0.03447100 \\
\hline $\mathrm{H}$ & 6.44453400 & 9.14314700 & 0.33094500 \\
\hline $\mathrm{H}$ & 6.07189100 & 7.85702200 & -0.82480100 \\
\hline $\mathrm{H}$ & 7.85860900 & 7.46525800 & 2.49451900 \\
\hline $\mathrm{H}$ & 6.62949300 & 8.71091000 & 2.74942300 \\
\hline $\mathrm{H}$ & 6.40654800 & 7.10432100 & 3.45059800 \\
\hline $\mathrm{H}$ & 8.45930000 & -7.17671800 & -2.05822800 \\
\hline $\mathrm{H}$ & 7.84166600 & -8.76361900 & -1.57766200 \\
\hline $\mathrm{H}$ & 8.33916600 & -7.59127400 & -0.34171300 \\
\hline $\mathrm{H}$ & 4.71149000 & -6.95766300 & -2.65360200 \\
\hline $\mathrm{H}$ & 6.28792100 & -6.79454100 & -3.44088100 \\
\hline $\mathrm{H}$ & 5.71878000 & -8.39208200 & -2.93285100 \\
\hline $\mathrm{H}$ & 6.03589000 & -7.91152100 & 0.75927800 \\
\hline $\mathrm{H}$ & 4.55662200 & -7.66588200 & -0.19165600 \\
\hline $\mathrm{H}$ & 5.61745900 & -9.03596500 & -0.53936300 \\
\hline $\mathrm{H}$ & -8.32763700 & 7.58895400 & 0.34217400 \\
\hline $\mathrm{H}$ & -7.82538700 & 8.76490700 & 1.57276700 \\
\hline $\mathrm{H}$ & -8.44036700 & 7.17910900 & 2.06032100 \\
\hline $\mathrm{H}$ & -6.02908500 & 7.90723900 & -0.76930400 \\
\hline $\mathrm{H}$ & -5.60558400 & 9.03526700 & 0.52457600 \\
\hline $\mathrm{H}$ & -4.54574500 & 7.66461800 & 0.17605300 \\
\hline $\mathrm{H}$ & -4.69002900 & 6.96303700 & 2.64053200 \\
\hline $\mathrm{H}$ & -6.26309000 & 6.80145000 & 3.43485900 \\
\hline $\mathrm{H}$ & -5.69670300 & 8.39781300 & 2.92011500 \\
\hline $\mathrm{H}$ & -4.38348800 & -6.90143500 & -2.67860500 \\
\hline
\end{tabular}




$\begin{array}{cccc}\mathrm{H} & -5.92390800 & -6.78549400 & -3.54200400 \\ \mathrm{H} & -5.30898900 & -8.37041600 & -3.04742200 \\ \mathrm{H} & -8.14396800 & -7.30409000 & -2.28409100 \\ \mathrm{H} & -7.47503600 & -8.87472500 & -1.81969600 \\ \mathrm{H} & -8.09053900 & -7.76690500 & -0.57707600 \\ \mathrm{H} & -4.32117000 & -7.67379600 & -0.23320300 \\ \mathrm{H} & -5.83367500 & -8.00630400 & 0.63562600 \\ \mathrm{H} & -5.30306800 & -9.07754100 & -0.66707900\end{array}$

$$
6^{\cdot+}
$$

Energy (with Zero Point Energy correction $)=-4409.208811(-4407.316458)$ Hartree Free energy $(298 \mathrm{~K})=-4407.462202$ Hartree

Zero number of imaginary frequency

$\begin{array}{rrrr}12 & & & \\ \mathrm{C} & 11.43203800 & 2.56412500 & -1.29079800 \\ \mathrm{C} & 11.44269400 & 1.31171800 & -0.65528100 \\ \mathrm{C} & 10.21042300 & 0.69899700 & -0.29107800 \\ \mathrm{C} & 8.98688000 & 1.40940100 & -0.47700800 \\ \mathrm{C} & 9.04003500 & 2.64245300 & -1.14044300 \\ \mathrm{C} & 10.24228200 & 3.23051800 & -1.57568100 \\ \mathrm{C} & 10.21401200 & -0.61091900 & 0.27329500 \\ \mathrm{C} & 8.99342200 & -1.30608800 & 0.50332600 \\ \mathrm{C} & 7.72924200 & -0.66538100 & 0.10966400 \\ \mathrm{C} & 7.72352300 & 0.76242300 & -0.07836400 \\ \mathrm{C} & 11.45228200 & -1.24050400 & 0.59463900 \\ \mathrm{C} & 11.44264700 & -2.48630200 & 1.23555500 \\ \mathrm{C} & 10.25200600 & -3.12996400 & 1.58527400 \\ \mathrm{C} & 9.04696200 & -2.53033500 & 1.19250200 \\ \mathrm{C} & 12.67465200 & 0.63153200 & -0.36633900 \\ \mathrm{C} & 12.68063400 & -0.57825100 & 0.25352600 \\ \mathrm{C} & 6.51688500 & -1.38404900 & -0.07191500 \\ \mathrm{C} & 5.28206200 & -0.67736300 & -0.11506000 \\ \mathrm{C} & 5.27410400 & 0.73983200 & 0.13479800 \\ \mathrm{C} & 6.50165300 & 1.46309700 & 0.09703100 \\ \mathrm{C} & 4.03883200 & -1.34156600 & -0.35906400 \\ \mathrm{C} & 2.84327000 & -0.68114100 & -0.20604400 \\ \mathrm{C} & 2.83468900 & 0.71309800 & 0.21501400 \\ \mathrm{C} & 4.02201900 & 1.38852700 & 0.37145800 \\ \mathrm{C} & -0.72129200 & 2.27813500 & 0.70937300 \\ \mathrm{C} & 0.70476400 & 2.28654400 & 0.71100900 \\ \mathrm{C} & 1.43563400 & 1.14390800 & 0.35606700 \\ \mathrm{C} & 0.68845900 & 0.00347800 & 0.00088100 \\ \mathrm{C} & -0.67711900 & -0.00438300 & -0.00078800 \\ \mathrm{C} & -1.43797500 & 1.12709200 & 0.35269100\end{array}$




\begin{tabular}{|c|c|c|c|}
\hline $\mathrm{C}$ & 1.44932000 & -1.12817200 & -0.35198800 \\
\hline $\mathrm{C}$ & 0.73265400 & -2.27921300 & -0.70859300 \\
\hline $\mathrm{C}$ & -0.69341900 & -2.28750500 & -0.71051600 \\
\hline $\mathrm{C}$ & -1.42430600 & -1.14484500 & -0.35578300 \\
\hline $\mathrm{C}$ & -2.83190100 & 0.67983600 & 0.20765300 \\
\hline $\mathrm{C}$ & -2.82338400 & -0.71419400 & -0.21415000 \\
\hline $\mathrm{C}$ & -4.01070100 & -1.38957400 & -0.37068400 \\
\hline $\mathrm{C}$ & -5.26262600 & -0.74136200 & -0.13199000 \\
\hline $\mathrm{C}$ & -5.27088900 & 0.67551900 & 0.12049600 \\
\hline $\mathrm{C}$ & -4.02746100 & 1.33986000 & 0.36281200 \\
\hline $\mathrm{C}$ & -6.48991700 & -1.46459000 & -0.09411500 \\
\hline $\mathrm{C}$ & -7.71100900 & -0.76459100 & 0.08926900 \\
\hline $\mathrm{C}$ & -7.71905700 & 0.66333000 & -0.09573700 \\
\hline $\mathrm{C}$ & -6.50611700 & 1.38204600 & 0.08172500 \\
\hline $\mathrm{C}$ & -8.97100000 & -1.41410400 & 0.49294400 \\
\hline $\mathrm{C}$ & -10.19664700 & -0.70670400 & 0.31121500 \\
\hline $\mathrm{C}$ & -10.20457100 & 0.60405500 & -0.25115900 \\
\hline $\mathrm{C}$ & -8.98612400 & 1.30270400 & -0.48301600 \\
\hline $\mathrm{C}$ & -11.44531000 & 1.23112600 & -0.56771800 \\
\hline $\mathrm{C}$ & -11.44061400 & 2.47862000 & -1.20542300 \\
\hline $\mathrm{C}$ & -10.25256700 & 3.12638700 & -1.55624700 \\
\hline $\mathrm{C}$ & -9.04483900 & 2.52876100 & -1.16846900 \\
\hline $\mathrm{C}$ & -9.01802600 & -2.64627800 & 1.15848500 \\
\hline $\mathrm{C}$ & -10.21711700 & -3.23697500 & 1.59881700 \\
\hline $\mathrm{C}$ & -11.40977200 & -2.57500100 & 1.31524800 \\
\hline $\mathrm{C}$ & -11.42606700 & -1.32302300 & 0.67871400 \\
\hline $\mathrm{C}$ & -12.66051800 & -0.64590700 & 0.39323600 \\
\hline $\mathrm{C}$ & -12.67103300 & 0.56480400 & -0.22486800 \\
\hline $\mathrm{C}$ & 6.46223100 & 2.93357000 & 0.35326600 \\
\hline $\mathrm{C}$ & 10.20491700 & 4.57057500 & -2.33371500 \\
\hline $\mathrm{C}$ & 11.60403800 & 5.01825100 & -2.79838000 \\
\hline $\mathrm{C}$ & 9.62885300 & 5.66712300 & -1.40713900 \\
\hline $\mathrm{C}$ & 9.30684700 & 4.43206200 & -3.58635100 \\
\hline $\mathrm{C}$ & 10.30350300 & -4.46000200 & 2.36073500 \\
\hline $\mathrm{C}$ & 8.90422300 & -4.96934200 & 2.75372100 \\
\hline $\mathrm{C}$ & 11.12432300 & -4.27251200 & 3.65924900 \\
\hline $\mathrm{C}$ & 10.98099300 & -5.53348300 & 1.47518300 \\
\hline $\mathrm{C}$ & 6.51191900 & -2.85414100 & -0.32634600 \\
\hline $\mathrm{C}$ & -6.50021800 & 2.85197900 & 0.33698300 \\
\hline $\mathrm{C}$ & -6.45602600 & -2.93292700 & -0.36249800 \\
\hline $\mathrm{C}$ & -10.30952400 & 4.45865700 & -2.32751500 \\
\hline $\mathrm{C}$ & -8.91263600 & 4.97257700 & -2.72304300 \\
\hline $\mathrm{C}$ & -10.98695000 & 5.52780500 & -1.43668600 \\
\hline $\mathrm{C}$ & -11.13370200 & 4.27318400 & -3.62418700 \\
\hline
\end{tabular}




\begin{tabular}{|c|c|c|c|}
\hline $\mathrm{C}$ & -10.17255500 & -4.57149600 & 2.36635900 \\
\hline $\mathrm{C}$ & -9.56967400 & -5.66896600 & 1.45820300 \\
\hline $\mathrm{C}$ & -11.57182200 & -5.03385900 & 2.81584900 \\
\hline $\mathrm{C}$ & -9.29316200 & -4.40958100 & 3.62955000 \\
\hline $\mathrm{C}$ & 5.72397100 & 3.81947300 & -0.45164900 \\
\hline $\mathrm{C}$ & 5.64263900 & 5.17350500 & -0.13819300 \\
\hline $\mathrm{C}$ & 6.27905500 & 5.71194300 & 0.99485300 \\
\hline $\mathrm{C}$ & 7.02088600 & 4.82223300 & 1.78576300 \\
\hline $\mathrm{C}$ & 7.11626300 & 3.46618300 & 1.47237100 \\
\hline $\mathrm{C}$ & 5.77478300 & -3.75358400 & 0.45784400 \\
\hline $\mathrm{C}$ & 5.75760800 & -5.11956200 & 0.16724000 \\
\hline $\mathrm{C}$ & 6.46305300 & -5.64780200 & -0.92321800 \\
\hline $\mathrm{C}$ & 7.19591200 & -4.73683500 & -1.70663800 \\
\hline $\mathrm{C}$ & 7.22696700 & -3.37778600 & -1.41743600 \\
\hline $\mathrm{C}$ & -5.72250600 & -3.82923000 & 0.42855200 \\
\hline $\mathrm{C}$ & -5.65185800 & -5.18643700 & 0.10595700 \\
\hline $\mathrm{C}$ & -6.29582700 & -5.70744100 & -1.02553000 \\
\hline $\mathrm{C}$ & -7.03373400 & -4.80117300 & -1.80896800 \\
\hline $\mathrm{C}$ & -7.12014800 & -3.45132600 & -1.48703700 \\
\hline $\mathrm{C}$ & -5.76644200 & 3.75172400 & -0.44998000 \\
\hline $\mathrm{C}$ & -5.74813200 & 5.11759800 & -0.15892900 \\
\hline $\mathrm{C}$ & -6.44896500 & 5.64535400 & 0.93473400 \\
\hline $\mathrm{C}$ & -7.17837300 & 4.73402900 & 1.72096600 \\
\hline $\mathrm{C}$ & -7.21058400 & 3.37508500 & 1.43136800 \\
\hline $\mathrm{C}$ & 6.12929800 & 7.20672300 & 1.32854700 \\
\hline $\mathrm{C}$ & 4.63494900 & 7.51904200 & 1.58560400 \\
\hline $\mathrm{C}$ & 6.63190100 & 8.06151500 & 0.14083900 \\
\hline $\mathrm{C}$ & 6.92901500 & 7.60897200 & 2.58216800 \\
\hline $\mathrm{C}$ & 6.45582500 & -7.14338000 & -1.28304400 \\
\hline $\mathrm{C}$ & 7.90523300 & -7.68548100 & -1.26529300 \\
\hline $\mathrm{C}$ & 5.85961900 & -7.32344400 & -2.70016300 \\
\hline $\mathrm{C}$ & 5.61757700 & -7.97875000 & -0.29703900 \\
\hline $\mathrm{C}$ & -6.44021200 & 7.14077900 & 1.29519100 \\
\hline $\mathrm{C}$ & -7.88973000 & 7.68276000 & 1.28463500 \\
\hline $\mathrm{C}$ & -5.60677100 & 7.97669100 & 0.30557800 \\
\hline $\mathrm{C}$ & -5.83724700 & 7.32021800 & 2.70953200 \\
\hline $\mathrm{C}$ & -6.21097900 & -7.18882300 & -1.43243100 \\
\hline $\mathrm{C}$ & -5.53842900 & -7.29381700 & -2.82283100 \\
\hline $\mathrm{C}$ & -7.63422800 & -7.79135600 & -1.50732000 \\
\hline $\mathrm{C}$ & -5.38750900 & -8.02370900 & -0.43360000 \\
\hline $\mathrm{H}$ & 12.38655600 & 2.99471100 & -1.57289500 \\
\hline $\mathrm{H}$ & 8.11579900 & 3.15884100 & -1.35277800 \\
\hline $\mathrm{H}$ & 12.39964500 & -2.93984400 & 1.47866000 \\
\hline $\mathrm{H}$ & 8.12024600 & -3.01807400 & 1.44905600 \\
\hline
\end{tabular}




\begin{tabular}{|c|c|c|c|}
\hline $\mathrm{H}$ & 13.60752700 & 1.11710100 & -0.64080300 \\
\hline $\mathrm{H}$ & 13.61796500 & -1.07480400 & 0.49053100 \\
\hline $\mathrm{H}$ & 4.04856900 & -2.38331300 & -0.65514900 \\
\hline $\mathrm{H}$ & 4.01630900 & 2.43104200 & 0.66464900 \\
\hline $\mathrm{H}$ & -1.23610700 & 3.19122900 & 0.99575500 \\
\hline $\mathrm{H}$ & 1.20821400 & 3.20559400 & 0.99852200 \\
\hline $\mathrm{H}$ & 1.24747400 & -3.19244000 & -0.99454300 \\
\hline $\mathrm{H}$ & -1.19687000 & -3.20660600 & -0.99785600 \\
\hline $\mathrm{H}$ & -4.00548900 & -2.43189500 & -0.66460200 \\
\hline $\mathrm{H}$ & -4.03698000 & 2.38134900 & 0.65983200 \\
\hline $\mathrm{H}$ & -12.39940900 & 2.93032800 & -1.44483500 \\
\hline $\mathrm{H}$ & -8.12017000 & 3.01972700 & -1.42621000 \\
\hline $\mathrm{H}$ & -8.09116400 & -3.15872800 & 1.36926200 \\
\hline $\mathrm{H}$ & -12.36221800 & -3.00826000 & 1.60025500 \\
\hline $\mathrm{H}$ & -13.59152000 & -1.13412700 & 0.66935900 \\
\hline $\mathrm{H}$ & -13.61024800 & 1.05926800 & -0.45877600 \\
\hline $\mathrm{H}$ & 12.28209500 & 5.18847300 & -1.95449300 \\
\hline $\mathrm{H}$ & 11.52296100 & 5.96278800 & -3.34704400 \\
\hline $\mathrm{H}$ & 12.06636100 & 4.28535000 & -3.46934400 \\
\hline $\mathrm{H}$ & 10.25481300 & 5.79521900 & -0.51655000 \\
\hline $\mathrm{H}$ & 9.59125100 & 6.62700400 & -1.93667400 \\
\hline $\mathrm{H}$ & 8.61481800 & 5.42784800 & -1.07297400 \\
\hline $\mathrm{H}$ & 9.69074900 & 3.65983700 & -4.26254900 \\
\hline $\mathrm{H}$ & 9.27873700 & 5.38028100 & -4.13600300 \\
\hline $\mathrm{H}$ & 8.27563800 & 4.17015700 & -3.32753000 \\
\hline $\mathrm{H}$ & 8.37392400 & -4.25134400 & 3.39032200 \\
\hline $\mathrm{H}$ & 8.28000600 & -5.17847100 & 1.87857900 \\
\hline $\mathrm{H}$ & 9.00136800 & -5.90182100 & 3.32067600 \\
\hline $\mathrm{H}$ & 10.66834900 & -3.51516600 & 4.30697900 \\
\hline $\mathrm{H}$ & 12.15467400 & -3.96318200 & 3.45657400 \\
\hline $\mathrm{H}$ & 11.16677200 & -5.21508700 & 4.21745800 \\
\hline $\mathrm{H}$ & 11.99996200 & -5.24693900 & 1.19395500 \\
\hline $\mathrm{H}$ & 11.04012100 & -6.48735300 & 2.01294900 \\
\hline $\mathrm{H}$ & 10.41188500 & -5.69635300 & 0.55277300 \\
\hline $\mathrm{H}$ & -8.38245600 & 4.25781800 & -3.36337500 \\
\hline $\mathrm{H}$ & -9.01370500 & 5.90654200 & -3.28685900 \\
\hline $\mathrm{H}$ & -8.28634500 & 5.18054800 & -1.84910900 \\
\hline $\mathrm{H}$ & -10.41545900 & 5.68927100 & -0.51550200 \\
\hline $\mathrm{H}$ & -12.00436800 & 5.23792400 & -1.15327500 \\
\hline $\mathrm{H}$ & -11.04999300 & 6.48315700 & -1.97136800 \\
\hline $\mathrm{H}$ & -10.67782800 & 3.51891400 & -4.27557000 \\
\hline $\mathrm{H}$ & -11.18003000 & 5.21735700 & -4.17937900 \\
\hline $\mathrm{H}$ & -12.16272100 & 3.96078400 & -3.41946200 \\
\hline $\mathrm{H}$ & -10.17862900 & -5.81070800 & 0.55811100 \\
\hline
\end{tabular}




\begin{tabular}{|c|c|c|c|}
\hline $\mathrm{H}$ & -9.53206800 & -6.62416900 & 1.99625300 \\
\hline $\mathrm{H}$ & -8.55227400 & -5.42306200 & 1.13927500 \\
\hline $\mathrm{H}$ & -12.05084500 & -4.30432800 & 3.47867500 \\
\hline $\mathrm{H}$ & -12.23741900 & -5.21471100 & 1.96424700 \\
\hline $\mathrm{H}$ & -11.48637100 & -5.97534000 & 3.36907900 \\
\hline $\mathrm{H}$ & -9.69824600 & -3.63883300 & 4.29497800 \\
\hline $\mathrm{H}$ & -8.26337700 & -4.13198100 & 3.38138800 \\
\hline $\mathrm{H}$ & -9.25649100 & -5.35319400 & 4.18665400 \\
\hline $\mathrm{H}$ & 5.21812400 & 3.44403500 & -1.33751300 \\
\hline $\mathrm{H}$ & 5.06460900 & 5.82054400 & -0.79186700 \\
\hline $\mathrm{H}$ & 7.53417500 & 5.17905000 & 2.67140600 \\
\hline $\mathrm{H}$ & 7.69411400 & 2.80892100 & 2.11578100 \\
\hline $\mathrm{H}$ & 5.22524900 & -3.38717900 & 1.32104400 \\
\hline $\mathrm{H}$ & 5.18174000 & -5.77307500 & 0.81288500 \\
\hline $\mathrm{H}$ & 7.75458500 & -5.09317700 & -2.56719900 \\
\hline $\mathrm{H}$ & 7.80393500 & -2.70768900 & -2.04805100 \\
\hline $\mathrm{H}$ & -5.21015500 & -3.46605100 & 1.31585300 \\
\hline $\mathrm{H}$ & -5.07813300 & -5.83792000 & 0.75560700 \\
\hline $\mathrm{H}$ & -7.55026300 & -5.15262600 & -2.69740600 \\
\hline $\mathrm{H}$ & -7.69543500 & -2.78348600 & -2.12172800 \\
\hline $\mathrm{H}$ & -5.22037200 & 3.38559200 & -1.31549300 \\
\hline $\mathrm{H}$ & -5.17503100 & 5.77140300 & -0.80673600 \\
\hline $\mathrm{H}$ & -7.73331900 & 5.08999900 & 2.58408900 \\
\hline $\mathrm{H}$ & -7.78477200 & 2.70468300 & 2.06419600 \\
\hline $\mathrm{H}$ & 4.01536200 & 7.29539900 & 0.71041700 \\
\hline $\mathrm{H}$ & 4.25041000 & 6.93435300 & 2.42921700 \\
\hline $\mathrm{H}$ & 4.50523300 & 8.58157600 & 1.82259100 \\
\hline $\mathrm{H}$ & 7.69223200 & 7.87232700 & -0.05966700 \\
\hline $\mathrm{H}$ & 6.51486500 & 9.12761900 & 0.36783500 \\
\hline $\mathrm{H}$ & 6.07438800 & 7.85637900 & -0.77907200 \\
\hline $\mathrm{H}$ & 8.00237200 & 7.42648000 & 2.45760900 \\
\hline $\mathrm{H}$ & 6.79731400 & 8.67924900 & 2.77409300 \\
\hline $\mathrm{H}$ & 6.59015700 & 7.07175400 & 3.47522600 \\
\hline $\mathrm{H}$ & 8.54752800 & -7.16268400 & -1.98155500 \\
\hline $\mathrm{H}$ & 7.91425400 & -8.74986300 & -1.52763600 \\
\hline $\mathrm{H}$ & 8.35352200 & -7.57814800 & -0.27093500 \\
\hline $\mathrm{H}$ & 4.82804800 & -6.95544700 & -2.74282300 \\
\hline $\mathrm{H}$ & 6.43851400 & -6.78660300 & -3.45886300 \\
\hline $\mathrm{H}$ & 5.85281500 & -8.38445700 & -2.97596200 \\
\hline $\mathrm{H}$ & 6.00374300 & -7.91285400 & 0.72653200 \\
\hline $\mathrm{H}$ & 4.56607000 & -7.66934800 & -0.28857200 \\
\hline $\mathrm{H}$ & 5.64649700 & -9.03320600 & -0.59180500 \\
\hline $\mathrm{H}$ & -8.34279400 & 7.57581800 & 0.29240100 \\
\hline $\mathrm{H}$ & -7.89756100 & 8.74702800 & 1.54747500 \\
\hline
\end{tabular}




$\begin{array}{cccc}\mathrm{H} & -8.52854100 & 7.15962900 & 2.00376300 \\ \mathrm{H} & -5.99773800 & 7.91110300 & -0.71619000 \\ \mathrm{H} & -5.63450800 & 9.03102600 & 0.60088900 \\ \mathrm{H} & -4.55525400 & 7.66750900 & 0.29204800 \\ \mathrm{H} & -4.80545100 & 6.95229700 & 2.74708400 \\ \mathrm{H} & -6.41247100 & 6.78294300 & 3.47071700 \\ \mathrm{H} & -5.82921100 & 8.38109600 & 2.98582000 \\ \mathrm{H} & -4.52232900 & -6.88326000 & -2.80097300 \\ \mathrm{H} & -6.10168800 & -6.75349100 & -3.59084300 \\ \mathrm{H} & -5.47364400 & -8.34310900 & -3.13383000 \\ \mathrm{H} & -8.26384900 & -7.26850100 & -2.23458900 \\ \mathrm{H} & -7.58253700 & -8.84370700 & -1.81018000 \\ \mathrm{H} & -8.13457800 & -7.74266600 & -0.53377500 \\ \mathrm{H} & -4.35063500 & -7.67501200 & -0.36432000 \\ \mathrm{H} & -5.82407400 & -8.00646200 & 0.57153100 \\ \mathrm{H} & -5.36045800 & -9.06814600 & -0.76229800\end{array}$

Mulliken spin densities:

$\begin{array}{rlc}1 & \mathrm{C} & 0.008019 \\ 2 & \mathrm{C} & -0.001500 \\ 3 & \mathrm{C} & 0.014509 \\ 4 & \mathrm{C} & 0.021620 \\ 5 & \mathrm{C} & -0.009516 \\ 6 & \mathrm{C} & 0.010708 \\ 7 & \mathrm{C} & 0.014386 \\ 8 & \mathrm{C} & 0.025216 \\ 9 & \mathrm{C} & -0.000119 \\ 10 & \mathrm{C} & -0.007087 \\ 11 & \mathrm{C} & -0.006753 \\ 12 & \mathrm{C} & 0.020362 \\ 13 & \mathrm{C} & 0.006796 \\ 14 & \mathrm{C} & -0.004184 \\ 15 & \mathrm{C} & -0.002655 \\ 16 & \mathrm{C} & 0.001562 \\ 17 & \mathrm{C} & 0.110684 \\ 18 & \mathrm{C} & -0.035938 \\ 19 & \mathrm{C} & -0.034085 \\ 20 & \mathrm{C} & 0.105234 \\ 21 & \mathrm{C} & 0.105542 \\ 22 & \mathrm{C} & -0.024606 \\ 23 & \mathrm{C} & -0.019879 \\ 24 & \mathrm{C} & 0.099620 \\ 25 & \mathrm{C} & 0.010909 \\ 26 & \mathrm{C} & 0.012092 \\ 27 & \mathrm{C} & 0.036082\end{array}$




\begin{tabular}{|c|c|c|}
\hline 28 & $\mathrm{C}$ & -0.014485 \\
\hline 29 & $\mathrm{C}$ & -0.014452 \\
\hline 30 & $\mathrm{C}$ & 0.038143 \\
\hline 31 & $\mathrm{C}$ & 0.038152 \\
\hline 32 & $\mathrm{C}$ & 0.010775 \\
\hline 33 & $\mathrm{C}$ & 0.012171 \\
\hline 34 & $\mathrm{C}$ & 0.035901 \\
\hline 35 & $\mathrm{C}$ & -0.024547 \\
\hline 36 & $\mathrm{C}$ & -0.019888 \\
\hline 37 & $\mathrm{C}$ & 0.099647 \\
\hline 38 & $\mathrm{C}$ & -0.034036 \\
\hline 39 & $\mathrm{C}$ & -0.036065 \\
\hline 40 & $\mathrm{C}$ & 0.105745 \\
\hline 41 & $\mathrm{C}$ & 0.105111 \\
\hline 42 & $\mathrm{C}$ & -0.006590 \\
\hline 43 & $\mathrm{C}$ & -0.000517 \\
\hline 44 & $\mathrm{C}$ & 0.110962 \\
\hline 45 & $\mathrm{C}$ & 0.021393 \\
\hline 46 & $\mathrm{C}$ & 0.014595 \\
\hline 47 & $\mathrm{C}$ & 0.014191 \\
\hline 48 & $\mathrm{C}$ & 0.025159 \\
\hline 49 & $\mathrm{C}$ & -0.006621 \\
\hline 50 & $\mathrm{C}$ & 0.020044 \\
\hline 51 & $\mathrm{C}$ & 0.006856 \\
\hline 52 & $\mathrm{C}$ & -0.004304 \\
\hline 53 & $\mathrm{C}$ & -0.009366 \\
\hline 54 & $\mathrm{C}$ & 0.010627 \\
\hline 55 & $\mathrm{C}$ & 0.008195 \\
\hline 56 & $\mathrm{C}$ & -0.001583 \\
\hline 57 & $\mathrm{C}$ & -0.002581 \\
\hline 58 & $\mathrm{C}$ & 0.001486 \\
\hline 59 & $\mathrm{C}$ & -0.007034 \\
\hline 60 & $\mathrm{C}$ & -0.000599 \\
\hline 61 & $\mathrm{C}$ & 0.000062 \\
\hline 62 & $\mathrm{C}$ & 0.000504 \\
\hline 63 & $\mathrm{C}$ & 0.000408 \\
\hline 64 & $\mathrm{C}$ & -0.000475 \\
\hline 65 & $\mathrm{C}$ & -0.000030 \\
\hline 66 & $\mathrm{C}$ & 0.000321 \\
\hline 67 & $\mathrm{C}$ & 0.000527 \\
\hline 68 & $\mathrm{C}$ & -0.006873 \\
\hline 69 & $\mathrm{C}$ & -0.006919 \\
\hline 70 & $\mathrm{C}$ & -0.006949 \\
\hline 71 & $\mathrm{C}$ & -0.000478 \\
\hline
\end{tabular}




\begin{tabular}{|c|c|c|}
\hline 72 & $\mathrm{C}$ & -0.000029 \\
\hline 73 & $\mathrm{C}$ & 0.000526 \\
\hline 74 & $\mathrm{C}$ & 0.000320 \\
\hline 75 & $\mathrm{C}$ & -0.000594 \\
\hline 76 & $\mathrm{C}$ & 0.000488 \\
\hline 77 & $\mathrm{C}$ & 0.000063 \\
\hline 78 & $\mathrm{C}$ & 0.000417 \\
\hline 79 & $\mathrm{C}$ & 0.008380 \\
\hline 80 & $\mathrm{C}$ & -0.004203 \\
\hline 81 & $\mathrm{C}$ & 0.009885 \\
\hline 82 & $\mathrm{C}$ & -0.003210 \\
\hline 83 & $\mathrm{C}$ & 0.008250 \\
\hline 84 & $\mathrm{C}$ & 0.010869 \\
\hline 85 & $\mathrm{C}$ & -0.005220 \\
\hline 86 & $\mathrm{C}$ & 0.012614 \\
\hline 87 & $\mathrm{C}$ & -0.004221 \\
\hline 88 & $\mathrm{C}$ & 0.009264 \\
\hline 89 & $\mathrm{C}$ & 0.009093 \\
\hline 90 & $\mathrm{C}$ & -0.004223 \\
\hline 91 & $\mathrm{C}$ & 0.009901 \\
\hline 92 & $\mathrm{C}$ & -0.003086 \\
\hline 93 & $\mathrm{C}$ & 0.007475 \\
\hline 94 & $\mathrm{C}$ & 0.010855 \\
\hline 95 & $\mathrm{C}$ & -0.005209 \\
\hline 96 & $\mathrm{C}$ & 0.012567 \\
\hline 97 & $\mathrm{C}$ & -0.004200 \\
\hline 98 & $\mathrm{C}$ & 0.009240 \\
\hline 99 & $\mathrm{C}$ & -0.000591 \\
\hline 100 & $\mathrm{C}$ & 0.000362 \\
\hline 101 & $\mathrm{C}$ & 0.000322 \\
\hline 102 & $\mathrm{C}$ & 0.000020 \\
\hline 103 & $\mathrm{C}$ & -0.000764 \\
\hline 104 & $\mathrm{C}$ & 0.000393 \\
\hline 105 & $\mathrm{C}$ & 0.000463 \\
\hline 106 & $\mathrm{C}$ & 0.000023 \\
\hline 107 & $\mathrm{C}$ & -0.000761 \\
\hline 108 & $\mathrm{C}$ & 0.000392 \\
\hline 109 & $\mathrm{C}$ & 0.000023 \\
\hline 110 & $\mathrm{C}$ & 0.000461 \\
\hline 111 & $\mathrm{C}$ & -0.000600 \\
\hline 112 & $\mathrm{C}$ & 0.000372 \\
\hline 113 & $\mathrm{C}$ & 0.000317 \\
\hline 114 & $\mathrm{C}$ & 0.000017 \\
\hline 115 & $\mathrm{H}$ & -0.000359 \\
\hline
\end{tabular}




$\begin{array}{ccc}116 & \mathrm{H} & 0.000236 \\ 117 & \mathrm{H} & -0.000880 \\ 118 & \mathrm{H} & -0.000051 \\ 119 & \mathrm{H} & 0.000093 \\ 120 & \mathrm{H} & -0.000074 \\ 121 & \mathrm{H} & -0.004032 \\ 122 & \mathrm{H} & -0.003830 \\ 123 & \mathrm{H} & -0.000778 \\ 124 & \mathrm{H} & -0.000822 \\ 125 & \mathrm{H} & -0.000772 \\ 126 & \mathrm{H} & -0.000824 \\ 127 & \mathrm{H} & -0.003832 \\ 128 & \mathrm{H} & -0.004039 \\ 129 & \mathrm{H} & -0.000866 \\ 130 & \mathrm{H} & -0.000044 \\ 131 & \mathrm{H} & 0.000222 \\ 132 & \mathrm{H} & -0.000367 \\ 133 & \mathrm{H} & 0.000090 \\ 134 & \mathrm{H} & -0.000071 \\ 135 & \mathrm{H} & 0.000010 \\ 136 & \mathrm{H} & -0.000020 \\ 137 & \mathrm{H} & 0.000016 \\ 138 & \mathrm{H} & -0.000007 \\ 139 & \mathrm{H} & 0.000094 \\ 140 & \mathrm{H} & -0.000049 \\ 141 & \mathrm{H} & 0.000006 \\ 142 & \mathrm{H} & 0.000018 \\ 143 & \mathrm{H} & -0.000024 \\ 144 & \mathrm{H} & 0.000021 \\ 145 & \mathrm{H} & -0.000021 \\ 146 & \mathrm{H} & -0.000012 \\ 157 & \mathrm{H} & -0.000001 \\ 158 & \mathrm{H} & 0.000299 \\ 159 & \mathrm{H} & -0.000001 \\ 149 & \mathrm{H} & -0.000002 \\ 150 & \mathrm{H} & 0.000221 \\ 151 & \mathrm{H} & -0.000008 \\ 152 & \mathrm{H} & -0.000300 \\ 153 & \mathrm{H} & 0.0000021 \\ 154 & \mathrm{H} & -0.000012 \\ 155 & \mathrm{H} & -0.000021 \\ & & -0.000034 \\ 156 & \mathrm{H} & -0.000009 \\ 159 & \end{array}$




$\begin{array}{ccc}160 & \mathrm{H} & 0.000220 \\ 161 & \mathrm{H} & -0.000002 \\ 162 & \mathrm{H} & -0.000006 \\ 163 & \mathrm{H} & 0.000087 \\ 164 & \mathrm{H} & -0.000049 \\ 165 & \mathrm{H} & 0.000016 \\ 166 & \mathrm{H} & 0.000011 \\ 167 & \mathrm{H} & -0.000020 \\ 168 & \mathrm{H} & 0.000006 \\ 169 & \mathrm{H} & -0.000024 \\ 170 & \mathrm{H} & 0.000020 \\ 171 & \mathrm{H} & -0.000459 \\ 172 & \mathrm{H} & 0.000362 \\ 173 & \mathrm{H} & 0.000240 \\ 174 & \mathrm{H} & -0.000308 \\ 175 & \mathrm{H} & -0.000589 \\ 176 & \mathrm{H} & 0.000393 \\ 177 & \mathrm{H} & 0.000290 \\ 178 & \mathrm{H} & -0.000365 \\ 179 & \mathrm{H} & -0.000493 \\ 180 & \mathrm{H} & 0.000347 \\ 181 & \mathrm{H} & 0.000244 \\ 182 & \mathrm{H} & -0.000271 \\ 183 & \mathrm{H} & -0.000588 \\ 184 & \mathrm{H} & 0.000393 \\ 185 & \mathrm{H} & 0.000289 \\ 186 & \mathrm{H} & -0.000363 \\ 187 & \mathrm{H} & -0.000013 \\ 188 & \mathrm{H} & -0.000002 \\ 189 & \mathrm{H} & 0.000138 \\ 190 & \mathrm{H} & -0.000013 \\ 191 & \mathrm{H} & 0.000052 \\ 192 & \mathrm{H} & -0.000017 \\ 193 & \mathrm{H} & 0.000006 \\ 194 & \mathrm{H} & -0.000009 \\ 195 & \mathrm{H} & 0.000006 \\ 196 & \mathrm{H} & -0.000016 \\ 197 & \mathrm{H} & 0.000134 \\ 198 & \mathrm{H} & -0.000004 \\ 199 & \mathrm{H} & -0.000006 \\ & \mathrm{H} & -0.000021 \\ & \mathrm{H} & 0.000141 \\ & 0.000011 \\ & 0.000006\end{array}$




$\begin{array}{ccc}204 & \mathrm{H} & -0.000006 \\ 205 & \mathrm{H} & -0.000004 \\ 206 & \mathrm{H} & 0.000134 \\ 207 & \mathrm{H} & -0.000016 \\ 208 & \mathrm{H} & 0.000011 \\ 209 & \mathrm{H} & -0.000006 \\ 210 & \mathrm{H} & 0.000006 \\ 211 & \mathrm{H} & -0.000006 \\ 212 & \mathrm{H} & -0.000021 \\ 213 & \mathrm{H} & 0.000140 \\ 214 & \mathrm{H} & -0.000005 \\ 215 & \mathrm{H} & -0.000017 \\ 216 & \mathrm{H} & 0.000113 \\ 217 & \mathrm{H} & -0.000012 \\ 218 & \mathrm{H} & 0.000101 \\ 219 & \mathrm{H} & -0.000009 \\ 220 & \mathrm{H} & 0.000005 \\ 221 & \mathrm{H} & 0.000008 \\ 222 & \mathrm{H} & -0.000004\end{array}$

\section{OS $6^{2 \cdot+}$}

Energy (with Zero Point Energy correction $)=-4408.927317$ (-4407.035481) Hartree Free energy $(298 \mathrm{~K})=-4407.180709$ Hartree

Zero number of imaginary frequency 21

$\begin{array}{lrrr}\mathrm{C} & 11.48449500 & 2.57474900 & -1.18754000 \\ \mathrm{C} & 11.46791500 & 1.32409400 & -0.54216700 \\ \mathrm{C} & 10.21560600 & 0.71625000 & -0.21816800 \\ \mathrm{C} & 9.00172900 & 1.42525700 & -0.45764200 \\ \mathrm{C} & 9.08384700 & 2.64956500 & -1.12236800 \\ \mathrm{C} & 10.30923800 & 3.23731300 & -1.51835300 \\ \mathrm{C} & 10.19502100 & -0.58414300 & 0.35174500 \\ \mathrm{C} & 8.96104800 & -1.28457800 & 0.52985600 \\ \mathrm{C} & 7.72874400 & -0.65713800 & 0.08860300 \\ \mathrm{C} & 7.72433400 & 0.77539600 & -0.09928400 \\ \mathrm{C} & 11.41612000 & -1.22072200 & 0.71172100 \\ \mathrm{C} & 11.37647500 & -2.46885200 & 1.35170200 \\ \mathrm{C} & 10.16790200 & -3.11874100 & 1.66722200 \\ \mathrm{C} & 8.98787200 & -2.52233500 & 1.23160200 \\ \mathrm{C} & 12.67962800 & 0.64314400 & -0.20627000 \\ \mathrm{C} & 12.65828200 & -0.57173100 & 0.41664200 \\ \mathrm{C} & 6.51260800 & -1.39411900 & -0.10707000 \\ \mathrm{C} & 5.27756700 & -0.69245400 & -0.13419500 \\ \mathrm{C} & 5.27427200 & 0.72319600 & 0.12984000 \\ \mathrm{C} & 6.50747600 & 1.46021600 & 0.08134800\end{array}$




\begin{tabular}{|c|c|c|c|}
\hline $\mathrm{C}$ & 4.03247000 & -1.35355500 & -0.39013900 \\
\hline $\mathrm{C}$ & 2.84076400 & -0.69484700 & -0.21845600 \\
\hline $\mathrm{C}$ & 2.83989900 & 0.69303500 & 0.21906900 \\
\hline $\mathrm{C}$ & 4.03213200 & 1.37053000 & 0.37157600 \\
\hline $\mathrm{C}$ & -0.70589100 & 2.27277000 & 0.74031800 \\
\hline $\mathrm{C}$ & 0.72398400 & 2.27211000 & 0.73907700 \\
\hline $\mathrm{C}$ & 1.44301900 & 1.13058200 & 0.36788900 \\
\hline $\mathrm{C}$ & 0.68835600 & -0.00137500 & -0.00026000 \\
\hline $\mathrm{C}$ & -0.67697200 & 0.00084200 & 0.00059300 \\
\hline $\mathrm{C}$ & -1.42721100 & 1.13313700 & 0.37002000 \\
\hline $\mathrm{C}$ & 1.43858000 & -1.13378700 & -0.36935500 \\
\hline $\mathrm{C}$ & 0.71728100 & -2.27341900 & -0.73958700 \\
\hline $\mathrm{C}$ & -0.71259600 & -2.27271700 & -0.73835500 \\
\hline $\mathrm{C}$ & -1.43164800 & -1.13119000 & -0.36723800 \\
\hline $\mathrm{C}$ & -2.82935100 & 0.69395100 & 0.21984300 \\
\hline $\mathrm{C}$ & -2.82850400 & -0.69381600 & -0.21778800 \\
\hline $\mathrm{C}$ & -4.02078300 & -1.37149400 & -0.36975300 \\
\hline $\mathrm{C}$ & -5.26269800 & -0.72456200 & -0.12636900 \\
\hline $\mathrm{C}$ & -5.26631500 & 0.69100400 & 0.13859800 \\
\hline $\mathrm{C}$ & -4.02115700 & 1.35240800 & 0.39279600 \\
\hline $\mathrm{C}$ & -6.49567800 & -1.46156800 & -0.07668400 \\
\hline $\mathrm{C}$ & -7.71182900 & -0.77712800 & 0.11071300 \\
\hline $\mathrm{C}$ & -7.71844200 & 0.65509200 & -0.07669300 \\
\hline $\mathrm{C}$ & -6.50179800 & 1.39243900 & 0.11443400 \\
\hline $\mathrm{C}$ & -8.98615800 & -1.42859600 & 0.47536900 \\
\hline $\mathrm{C}$ & -10.20220200 & -0.72260500 & 0.23873500 \\
\hline $\mathrm{C}$ & -10.18570500 & 0.57754200 & -0.33185800 \\
\hline $\mathrm{C}$ & -8.95357000 & 1.28073800 & -0.51328700 \\
\hline $\mathrm{C}$ & -11.40927300 & 1.21119700 & -0.68861800 \\
\hline $\mathrm{C}$ & -11.37419000 & 2.45955200 & -1.32823500 \\
\hline $\mathrm{C}$ & -10.16793400 & 3.11252000 & -1.64621600 \\
\hline $\mathrm{C}$ & -8.98523100 & 2.51873500 & -1.21401900 \\
\hline $\mathrm{C}$ & -9.06256800 & -2.65069200 & 1.14480900 \\
\hline $\mathrm{C}$ & -10.28508600 & -3.23969200 & 1.54747100 \\
\hline $\mathrm{C}$ & -11.46318000 & -2.58186500 & 1.21651500 \\
\hline $\mathrm{C}$ & -11.45194400 & -1.33304700 & 0.56740900 \\
\hline $\mathrm{C}$ & -12.66619400 & -0.65540300 & 0.23352400 \\
\hline $\mathrm{C}$ & -12.64915500 & 0.55899500 & -0.39032400 \\
\hline $\mathrm{C}$ & 6.45167300 & 2.93300600 & 0.31900000 \\
\hline $\mathrm{C}$ & 10.29662200 & 4.57026800 & -2.28555900 \\
\hline $\mathrm{C}$ & 11.71240800 & 5.02547800 & -2.68732400 \\
\hline $\mathrm{C}$ & 9.66780400 & 5.66666600 & -1.39273600 \\
\hline $\mathrm{C}$ & 9.45684700 & 4.41115400 & -3.57650300 \\
\hline $\mathrm{C}$ & 10.20129800 & -4.43628300 & 2.46022400 \\
\hline
\end{tabular}




\begin{tabular}{|c|c|c|c|}
\hline $\mathrm{C}$ & 8.79067900 & -4.95748400 & 2.78966200 \\
\hline $\mathrm{C}$ & 10.95728100 & -4.21550600 & 3.79330600 \\
\hline $\mathrm{C}$ & 10.93764900 & -5.50870600 & 1.62052900 \\
\hline $\mathrm{C}$ & 6.54672000 & -2.84939100 & -0.38420800 \\
\hline $\mathrm{C}$ & -6.53511700 & 2.84787000 & 0.39059000 \\
\hline $\mathrm{C}$ & -6.44507400 & -2.93285100 & -0.32359900 \\
\hline $\mathrm{C}$ & -10.20643400 & 4.43060100 & -2.43811200 \\
\hline $\mathrm{C}$ & -8.79789700 & 4.95553800 & -2.77054400 \\
\hline $\mathrm{C}$ & -10.94336000 & 5.50061000 & -1.59583800 \\
\hline $\mathrm{C}$ & -10.96507400 & 4.20903700 & -3.76955500 \\
\hline $\mathrm{C}$ & -10.26617000 & -4.56558500 & 2.32688200 \\
\hline $\mathrm{C}$ & -9.61501600 & -5.66516500 & 1.45431000 \\
\hline $\mathrm{C}$ & -11.68119400 & -5.03259600 & 2.71756600 \\
\hline $\mathrm{C}$ & -9.44267400 & -4.38220300 & 3.62522800 \\
\hline $\mathrm{C}$ & 5.72813700 & 3.80076000 & -0.51833300 \\
\hline $\mathrm{C}$ & 5.63874200 & 5.15998900 & -0.23048700 \\
\hline $\mathrm{C}$ & 6.24842100 & 5.71977000 & 0.90750700 \\
\hline $\mathrm{C}$ & 6.97299600 & 4.84533000 & 1.73132100 \\
\hline $\mathrm{C}$ & 7.07887800 & 3.48419000 & 1.44408100 \\
\hline $\mathrm{C}$ & 5.75260200 & -3.77582100 & 0.31603900 \\
\hline $\mathrm{C}$ & 5.78379200 & -5.13312300 & 0.00038300 \\
\hline $\mathrm{C}$ & 6.58638200 & -5.63118600 & -1.03970200 \\
\hline $\mathrm{C}$ & 7.37596600 & -4.69633400 & -1.73735800 \\
\hline $\mathrm{C}$ & 7.37095500 & -3.34618800 & -1.41547100 \\
\hline $\mathrm{C}$ & -5.72508300 & -3.80897800 & 0.50166700 \\
\hline $\mathrm{C}$ & -5.64652700 & -5.17236700 & 0.20822800 \\
\hline $\mathrm{C}$ & -6.26485000 & -5.71791000 & -0.92656400 \\
\hline $\mathrm{C}$ & -6.98658400 & -4.82904300 & -1.74492200 \\
\hline $\mathrm{C}$ & -7.08350200 & -3.47315400 & -1.45239400 \\
\hline $\mathrm{C}$ & -5.74339800 & 3.77358600 & -0.31327800 \\
\hline $\mathrm{C}$ & -5.77383000 & 5.13125800 & 0.00090500 \\
\hline $\mathrm{C}$ & -6.57309300 & 5.63033400 & 1.04304000 \\
\hline $\mathrm{C}$ & -7.36014100 & 4.69611700 & 1.74444000 \\
\hline $\mathrm{C}$ & -7.35598300 & 3.34561800 & 1.42406200 \\
\hline $\mathrm{C}$ & 6.08833000 & 7.21944500 & 1.21232800 \\
\hline $\mathrm{C}$ & 4.58723200 & 7.53066900 & 1.42970600 \\
\hline $\mathrm{C}$ & 6.61535400 & 8.05452000 & 0.02097500 \\
\hline $\mathrm{C}$ & 6.85858700 & 7.64444900 & 2.47680200 \\
\hline $\mathrm{C}$ & 6.62432200 & -7.11677000 & -1.43005400 \\
\hline $\mathrm{C}$ & 8.07327600 & -7.64625600 & -1.29840800 \\
\hline $\mathrm{C}$ & 6.15606700 & -7.26522400 & -2.89893800 \\
\hline $\mathrm{C}$ & 5.71067400 & -7.98032100 & -0.54003300 \\
\hline $\mathrm{C}$ & -6.61014900 & 7.11637400 & 1.43175100 \\
\hline $\mathrm{C}$ & -8.05967300 & 7.64529700 & 1.30416500 \\
\hline
\end{tabular}


C

C

C

C

C

C

H

$\mathrm{H}$

$\mathrm{H}$

$\mathrm{H}$

$\mathrm{H}$

$\mathrm{H}$

$\mathrm{H}$

$\mathrm{H}$

$\mathrm{H}$

$\mathrm{H}$

$\mathrm{H}$

$\mathrm{H}$

$\mathrm{H}$

$\mathrm{H}$

$\mathrm{H}$

$\mathrm{H}$

$\mathrm{H}$

$\mathrm{H}$

$\mathrm{H}$

$\mathrm{H}$

$\mathrm{H}$

$\mathrm{H}$

$\mathrm{H}$

$\mathrm{H}$

$\mathrm{H}$

$\mathrm{H}$

$\mathrm{H}$

$\mathrm{H}$

$\mathrm{H}$

$\mathrm{H}$

$\mathrm{H}$

$\mathrm{H}$

$\mathrm{H}$

$\mathrm{H}$

$\mathrm{H}$

$\mathrm{H}$

$\mathrm{H}$

$\mathrm{H}$

$\begin{array}{rrc}-5.69963900 & 7.97910000 & 0.53772500 \\ -6.13716300 & 7.26672600 & 2.89892100 \\ -6.16707800 & -7.20667500 & -1.30157400 \\ -5.46022500 & -7.33567600 & -2.67308400 \\ -7.58725900 & -7.81346700 & -1.40022800 \\ -5.36684400 & -8.01904900 & -0.26602500 \\ 12.44879300 & 3.00240800 & -1.43694800 \\ 8.17009400 & 3.17054300 & -1.36851300 \\ 12.32191100 & -2.92401800 & 1.63245200 \\ 8.04897100 & -2.99713000 & 1.46680300 \\ 13.62541600 & 1.12059400 & -0.44665100 \\ 13.58447600 & -1.07225700 & 0.68431800 \\ 4.04055600 & -2.38605100 & -0.71615900 \\ 4.02761600 & 2.41456300 & 0.65839600 \\ -1.21249100 & 3.18587100 & 1.04020500 \\ 1.23295100 & 3.18425400 & 1.03780800 \\ 1.22389100 & -3.18658400 & -1.03926100 \\ -1.22157500 & -3.18490200 & -1.03693000 \\ -4.01665500 & -2.41549500 & -0.65672800 \\ -4.02908500 & 2.38487400 & 0.71894000 \\ -12.32136400 & 2.91264600 & -1.60646400 \\ -8.04815400 & 2.99614700 & -1.45121600 \\ -8.14632100 & -3.16775200 & 1.39017400 \\ -12.42560500 & -3.01140700 & 1.46991000 \\ -13.61028800 & -1.13477600 & 0.47673300 \\ -13.57719500 & 1.05713000 & -0.65607200 \\ 12.34995600 & 5.20531100 & -1.81441300 \\ 11.64916400 & 5.96688700 & -3.24255700 \\ 12.20989500 & 4.29509700 & -3.33537600 \\ 10.25074900 & 5.80845100 & -0.47566300 \\ 9.64831900 & 6.62052400 & -1.93260800 \\ 8.64022000 & 5.42399000 & -1.10528300 \\ 9.87894100 & 3.63951000 & -4.22994700 \\ 9.44615200 & 5.35542000 & -4.13228600 \\ 8.41654300 & 4.14265000 & -3.36407600 \\ 8.22311000 & -4.24304200 & 3.39760700 \\ 8.21165500 & -5.18071800 & 1.88694900 \\ 8.87146000 & -5.88459000 & 3.36647900 \\ 10.46036300 & -3.45779200 & 4.40939500 \\ 11.99289600 & -3.89643300 & 3.63697000 \\ 10.98601200 & -5.15003700 & 4.36422300 \\ 11.96596800 & -5.21425900 & 1.38502000 \\ 10.98513400 & -6.45178300 & 2.17647400 \\ 10.41604900 & -5.69598800 & 0.67511600\end{array}$




\begin{tabular}{|c|c|c|c|}
\hline $\mathrm{H}$ & -8.23005600 & 4.24301100 & -3.38047500 \\
\hline $\mathrm{H}$ & -8.88235400 & 5.88294100 & -3.34636200 \\
\hline $\mathrm{H}$ & -8.21719900 & 5.17940700 & -1.86906200 \\
\hline $\mathrm{H}$ & -10.41988600 & 5.68852400 & -0.65158700 \\
\hline $\mathrm{H}$ & -11.97035400 & 5.20344100 & -1.35799400 \\
\hline $\mathrm{H}$ & -10.99454000 & 6.44396800 & -2.15098300 \\
\hline $\mathrm{H}$ & -10.46780700 & 3.45299100 & -4.38741100 \\
\hline $\mathrm{H}$ & -10.99742500 & 5.14393300 & -4.33968800 \\
\hline $\mathrm{H}$ & -11.99953100 & 3.88733600 & -3.61097700 \\
\hline $\mathrm{H}$ & -10.18250000 & -5.82120500 & 0.53005600 \\
\hline $\mathrm{H}$ & -9.59641900 & -6.61338600 & 2.00417300 \\
\hline $\mathrm{H}$ & -8.58498400 & -5.41769100 & 1.17971500 \\
\hline $\mathrm{H}$ & -12.19261400 & -4.30341200 & 3.35600700 \\
\hline $\mathrm{H}$ & -12.30795800 & -5.22424100 & 1.83934100 \\
\hline $\mathrm{H}$ & -11.61400800 & -5.96963900 & 3.27967800 \\
\hline $\mathrm{H}$ & -9.88231800 & -3.60996400 & 4.26626200 \\
\hline $\mathrm{H}$ & -8.40415900 & -4.10082900 & 3.42057000 \\
\hline $\mathrm{H}$ & -9.42478700 & -5.32062500 & 4.19071400 \\
\hline $\mathrm{H}$ & 5.24089300 & 3.40903500 & -1.40774100 \\
\hline $\mathrm{H}$ & 5.07496100 & 5.79452600 & -0.90804000 \\
\hline $\mathrm{H}$ & 7.46327000 & 5.21901200 & 2.62282100 \\
\hline $\mathrm{H}$ & 7.64235500 & 2.84006000 & 2.11325000 \\
\hline $\mathrm{H}$ & 5.13457800 & -3.43682800 & 1.14276800 \\
\hline $\mathrm{H}$ & 5.17064200 & -5.80956800 & 0.58460000 \\
\hline $\mathrm{H}$ & 8.00803900 & -5.02887300 & -2.55492300 \\
\hline $\mathrm{H}$ & 7.98795500 & -2.65753000 & -1.98464400 \\
\hline $\mathrm{H}$ & -5.23049000 & -3.42695800 & 1.39130000 \\
\hline $\mathrm{H}$ & -5.08637700 & -5.80987700 & 0.88283900 \\
\hline $\mathrm{H}$ & -7.48088700 & -5.19955700 & -2.63793800 \\
\hline $\mathrm{H}$ & -7.64536200 & -2.82016700 & -2.11422700 \\
\hline $\mathrm{H}$ & -5.12792000 & 3.43368100 & -1.14153100 \\
\hline $\mathrm{H}$ & -5.16273100 & 5.80714900 & -0.58609300 \\
\hline $\mathrm{H}$ & -7.98952700 & 5.02948500 & 2.56373800 \\
\hline $\mathrm{H}$ & -7.97099200 & 2.65748500 & 1.99601400 \\
\hline $\mathrm{H}$ & 3.98819600 & 7.29254100 & 0.54397100 \\
\hline $\mathrm{H}$ & 4.18478400 & 6.95973100 & 2.27442900 \\
\hline $\mathrm{H}$ & 4.45078700 & 8.59629000 & 1.64626300 \\
\hline $\mathrm{H}$ & 7.68101000 & 7.86743000 & -0.15120600 \\
\hline $\mathrm{H}$ & 6.48955300 & 9.12335500 & 0.22740600 \\
\hline $\mathrm{H}$ & 6.07973600 & 7.83282300 & -0.90811800 \\
\hline $\mathrm{H}$ & 7.93508400 & 7.46348400 & 2.38018100 \\
\hline $\mathrm{H}$ & 6.72073800 & 8.71729300 & 2.64673200 \\
\hline $\mathrm{H}$ & 6.50017000 & 7.12323500 & 3.37172500 \\
\hline $\mathrm{H}$ & 8.77069600 & -7.10420900 & -1.94546100 \\
\hline
\end{tabular}




$\begin{array}{lrrr}\mathrm{H} & 8.11275000 & -8.70343600 & -1.58343200 \\ \mathrm{H} & 8.43256800 & -7.56170500 & -0.26641300 \\ \mathrm{H} & 5.12901100 & -6.90416300 & -3.02465200 \\ \mathrm{H} & 6.79567600 & -6.71006600 & -3.59278200 \\ \mathrm{H} & 6.18271100 & -8.31981500 & -3.19494500 \\ \mathrm{H} & 6.00637800 & -7.93962400 & 0.51456200 \\ \mathrm{H} & 4.65946300 & -7.67935200 & -0.61637300 \\ \mathrm{H} & 5.77427600 & -9.02625100 & -0.85688500 \\ \mathrm{H} & -8.42229600 & 7.55936700 & 0.27345200 \\ \mathrm{H} & -8.09852200 & 8.70281700 & 1.58801400 \\ \mathrm{H} & -8.75482700 & 7.10384500 & 1.95414800 \\ \mathrm{H} & -5.99877100 & 7.93705000 & -0.51584900 \\ \mathrm{H} & -5.76249600 & 9.02539400 & 0.85352600 \\ \mathrm{H} & -4.64810400 & 7.67850200 & 0.61100000 \\ \mathrm{H} & -5.10959100 & 6.90612500 & 3.02171400 \\ \mathrm{H} & -6.77433700 & 6.71221100 & 3.59551600 \\ \mathrm{H} & -6.16315800 & 8.32166300 & 3.19375300 \\ \mathrm{H} & -4.44587400 & -6.92187000 & -2.63492400 \\ \mathrm{H} & -6.00636100 & -6.81428700 & -3.46614900 \\ \mathrm{H} & -5.38484300 & -8.39059800 & -2.96026200 \\ \mathrm{H} & -8.20020100 & -7.30746900 & -2.15325500 \\ \mathrm{H} & -7.52476200 & -8.87064700 & -1.68187400 \\ \mathrm{H} & -8.11162100 & -7.74983700 & -0.44028100 \\ \mathrm{H} & -4.33197000 & -7.66811800 & -0.17906200 \\ \mathrm{H} & -5.82764100 & -7.98352100 & 0.72775800 \\ \mathrm{H} & -5.33064400 & -9.06948200 & -0.57280300\end{array}$

Mulliken spin densities:
$\begin{array}{lll}1 & \mathrm{C} & -0.030917\end{array}$
$\begin{array}{lll}2 & \mathrm{C} & 0.041229\end{array}$
3 C 0.034697
$\begin{array}{lll}4 & \mathrm{C} & 0.001069\end{array}$
$\begin{array}{lll}5 & \mathrm{C} & 0.012816\end{array}$
$\begin{array}{lll}6 & \mathrm{C} & 0.033218\end{array}$
$\begin{array}{lll}7 & \mathrm{C} & 0.014295\end{array}$
8 C 0.091080
$\begin{array}{lll}9 & \mathrm{C} & 0.051564\end{array}$
10 C -0.041159
11 C -0.057597
$\begin{array}{lll}12 & \mathrm{C} & 0.203089\end{array}$
13 C -0.037836
14 C 0.075332
15 C -0.017570
16 C 0.068603
17 C 0.173321 


$\begin{array}{llc}18 & \text { C } & -0.050548 \\ 19 & \text { C } & 0.017588 \\ 20 & \text { C } & 0.076842 \\ 21 & \text { C } & 0.072605 \\ 22 & \text { C } & -0.025156 \\ 23 & \text { C } & 0.069064 \\ 24 & \text { C } & 0.015430 \\ 25 & \text { C } & -0.038808 \\ 26 & \text { C } & 0.059059 \\ 27 & \text { C } & -0.035833 \\ 28 & \text { C } & 0.021380 \\ 29 & \text { C } & -0.021225 \\ 30 & \text { C } & 0.054459 \\ 31 & \text { C } & -0.054646 \\ 32 & \text { C } & 0.038626 \\ 33 & \text { C } & -0.059167 \\ 34 & \text { C } & 0.035568 \\ 35 & \text { C } & 0.025138 \\ 36 & \text { C } & -0.068891 \\ 37 & \text { C } & -0.016394 \\ 38 & \text { C } & -0.016937 \\ 39 & \text { C } & 0.050840 \\ 40 & \text { C } & -0.073408 \\ 41 & \text { C } & -0.078417 \\ 42 & \text { C } & 0.041607 \\ 43 & \text { C } & -0.050729 \\ 44 & \text { C } & -0.174661 \\ 45 & \text { C } & -0.001727 \\ 46 & \text { C } & -0.034765 \\ 47 & \text { C } & -0.014522 \\ 48 & \text { C } & -0.090997 \\ 49 & \text { C } & 0.057260 \\ 50 & \text { C } & -0.201216 \\ 51 & \text { C } & 0.036956 \\ 52 & \text { C } & -0.073862 \\ 53 & \text { C } & -0.011636 \\ 54 & \text { C } & -0.033723 \\ 55 & \text { C } & 0.031229 \\ 56 & \text { C } & -0.041030 \\ 57 & \text { C } & 0.017817 \\ 58 & \text { C } & -0.067965 \\ 59 & \text { C } & -0.005430 \\ 60 & \text { C } & -0.001852 \\ 61 & \text { C } & -0.000040\end{array}$




$$
\begin{aligned}
& \begin{array}{lll}
62 & \mathrm{C} & 0.001451
\end{array} \\
& 63 \mathrm{C} \quad 0.001343 \\
& 64 \mathrm{C} \quad 0.001713 \\
& 65 \text { C }-0.000021 \\
& \begin{array}{lll}
66 & \mathrm{C} & 0.000309
\end{array} \\
& 67 \text { C } 0.000650 \\
& 68 \mathrm{C} \quad 0.001734 \\
& 69 \text { C }-0.001513 \\
& \begin{array}{lll}
70 & \mathrm{C} & 0.005469
\end{array} \\
& \begin{array}{lll}
71 & \mathrm{C} & -0.001668
\end{array} \\
& \begin{array}{lll}
72 & \mathrm{C} & 0.000019
\end{array} \\
& 73 \text { C }-0.000666 \\
& 74 \text { C }-0.000325 \\
& \begin{array}{lll}
75 & \mathrm{C} & 0.001879
\end{array} \\
& \begin{array}{lll}
76 & \mathrm{C} & -0.001435
\end{array} \\
& \begin{array}{lll}
77 & \mathrm{C} & 0.000041
\end{array} \\
& \begin{array}{lll}
78 & \mathrm{C} & -0.001388
\end{array} \\
& 79 \text { C } 0.004952 \\
& 80 \text { C }-0.001723 \\
& 81 \text { C } 0.004726 \\
& 82 \text { C }-0.000614 \\
& 83 \text { C } 0.003443 \\
& 84 \text { C } 0.026547 \\
& 85 \text { C }-0.010250 \\
& 86 \text { C } 0.046720 \\
& 87 \text { C }-0.018710 \\
& 88 \text { C } 0.032255 \\
& 89 \text { C }-0.005486 \\
& 90 \mathrm{C} \quad 0.001818 \\
& 91 \mathrm{C} \quad-0.004984 \\
& 92 \text { C } 0.000543 \\
& 93 \text { C }-0.003214 \\
& 94 \text { C }-0.026612 \\
& 95 \mathrm{C} \quad 0.010310 \\
& 96 \text { C }-0.046676 \\
& 97 \text { C } 0.018673 \\
& 98 \text { C }-0.032226 \\
& 99 \text { C } \quad-0.000271 \\
& 100 \mathrm{C} \quad 0.000204 \\
& 101 \text { C } 0.000204 \\
& 102 \text { C } 0.000009 \\
& 103 \text { C }-0.002709 \\
& 104 \text { C } 0.001689 \\
& 105 \text { C } 0.001928
\end{aligned}
$$




$\begin{array}{ccc}106 & \mathrm{C} & 0.000099 \\ 107 & \mathrm{C} & 0.002707 \\ 108 & \mathrm{C} & -0.001687 \\ 109 & \mathrm{C} & -0.000099 \\ 110 & \mathrm{C} & -0.001925 \\ 111 & \mathrm{C} & 0.000282 \\ 112 & \mathrm{C} & -0.000217 \\ 113 & \mathrm{C} & -0.000222 \\ 114 & \mathrm{C} & -0.000005 \\ 115 & \mathrm{H} & 0.001052 \\ 116 & \mathrm{H} & -0.000607 \\ 117 & \mathrm{H} & -0.008592 \\ 118 & \mathrm{H} & -0.003889 \\ 119 & \mathrm{H} & 0.000537 \\ 120 & \mathrm{H} & -0.002809 \\ 121 & \mathrm{H} & -0.002567 \\ 122 & \mathrm{H} & -0.000716 \\ 123 & \mathrm{H} & 0.001398 \\ 124 & \mathrm{H} & -0.002526 \\ 125 & \mathrm{H} & -0.001388 \\ 126 & \mathrm{H} & 0.002534 \\ 127 & \mathrm{H} & 0.000752 \\ 128 & \mathrm{H} & 0.002595 \\ 129 & \mathrm{H} & 0.008512 \\ 130 & \mathrm{H} & 0.003827 \\ 131 & \mathrm{H} & 0.000565 \\ 132 & \mathrm{H} & -0.001066 \\ 133 & \mathrm{H} & -0.000549 \\ 134 & \mathrm{H} & 0.002783 \\ 135 & \mathrm{H} & 0.000034 \\ 146 & \mathrm{H} & -0.000030 \\ 148 & \mathrm{H} & -0.000032 \\ 149 & \mathrm{H} & 0.000064 \\ 147 & \mathrm{H} & -0.0000634 \\ 138 & \mathrm{H} & -0.000014 \\ 139 & \mathrm{H} & 0.000658 \\ 140 & \mathrm{H} & -0.000069 \\ 141 & \mathrm{H} & -0.000008 \\ 142 & \mathrm{H} & 0.000606 \\ & & -0.000048 \\ 145 & \mathrm{H} & -0.000068 \\ 146 & \end{array}$




$\begin{array}{ccc}150 & \mathrm{H} & 0.000046 \\ 151 & \mathrm{H} & 0.000809 \\ 152 & \mathrm{H} & -0.000107 \\ 153 & \mathrm{H} & -0.000060 \\ 154 & \mathrm{H} & 0.000030 \\ 155 & \mathrm{H} & 0.000068 \\ 156 & \mathrm{H} & 0.000106 \\ 157 & \mathrm{H} & -0.000045 \\ 158 & \mathrm{H} & -0.000813 \\ 159 & \mathrm{H} & 0.000032 \\ 160 & \mathrm{H} & -0.000691 \\ 161 & \mathrm{H} & -0.000062 \\ 162 & \mathrm{H} & 0.000012 \\ 163 & \mathrm{H} & -0.000646 \\ 164 & \mathrm{H} & 0.000067 \\ 165 & \mathrm{H} & -0.000040 \\ 166 & \mathrm{H} & -0.000035 \\ 167 & \mathrm{H} & 0.000035 \\ 168 & \mathrm{H} & 0.000009 \\ 169 & \mathrm{H} & 0.000050 \\ 170 & \mathrm{H} & -0.000617 \\ 171 & \mathrm{H} & -0.000249 \\ 172 & \mathrm{H} & 0.000253 \\ 173 & \mathrm{H} & 0.000151 \\ 174 & \mathrm{H} & -0.000092 \\ 175 & \mathrm{H} & -0.001313 \\ 176 & \mathrm{H} & 0.000613 \\ 177 & \mathrm{H} & 0.000881 \\ 178 & \mathrm{H} & -0.001464 \\ 179 & \mathrm{H} & 0.000275 \\ 180 & \mathrm{H} & -0.000251 \\ 181 & \mathrm{H} & -0.000158 \\ 182 & \mathrm{H} & 0.000080 \\ 183 & \mathrm{H} & 0.001316 \\ 184 & \mathrm{H} & -0.000618 \\ 185 & \mathrm{H} & -0.000882 \\ 186 & \mathrm{H} & 0.001462 \\ 187 & \mathrm{H} & -0.000009 \\ 188 & \mathrm{H} & 0.000003 \\ 189 & \mathrm{H} & 0.000063 \\ 193 & \mathrm{H} & -0.000014 \\ & \mathrm{H} & 0.000026 \\ & & -0.000011 \\ & \end{array}$




$\begin{array}{ccc}194 & \mathrm{H} & 0.000001 \\ 195 & \mathrm{H} & 0.000007 \\ 196 & \mathrm{H} & -0.000071 \\ 197 & \mathrm{H} & 0.000478 \\ 198 & \mathrm{H} & -0.000026 \\ 199 & \mathrm{H} & -0.000020 \\ 200 & \mathrm{H} & -0.000084 \\ 201 & \mathrm{H} & 0.000501 \\ 202 & \mathrm{H} & 0.000047 \\ 203 & \mathrm{H} & 0.000027 \\ 204 & \mathrm{H} & -0.000043 \\ 205 & \mathrm{H} & 0.000026 \\ 206 & \mathrm{H} & -0.000478 \\ 207 & \mathrm{H} & 0.000070 \\ 208 & \mathrm{H} & -0.000047 \\ 209 & \mathrm{H} & 0.000043 \\ 210 & \mathrm{H} & -0.000027 \\ 211 & \mathrm{H} & 0.000020 \\ 212 & \mathrm{H} & 0.000084 \\ 213 & \mathrm{H} & -0.000500 \\ 214 & \mathrm{H} & 0.000001 \\ 215 & \mathrm{H} & 0.000010 \\ 216 & \mathrm{H} & -0.000054 \\ 217 & \mathrm{H} & 0.000007 \\ 218 & \mathrm{H} & -0.000070 \\ 219 & \mathrm{H} & 0.000013 \\ 220 & \mathrm{H} & -0.000002 \\ 221 & \mathrm{H} & -0.000006 \\ 222 & \mathrm{H} & 0.000002\end{array}$

\section{$\mathrm{CS}^{2+}$}

Energy(with Zero Point Energy correction) $=-4408.933671$ (-4407.041778) Hartree Free energy $(298 \mathrm{~K})=-4407.186279$ Hartree

Zero number of imaginary frequency

21

C

$\begin{array}{rrr}-11.43460600 & 2.54177900 & 1.29849700 \\ -11.44447600 & 1.30385400 & 0.64088000 \\ -10.20572600 & 0.69203200 & 0.28378400 \\ -8.97913200 & 1.40095700 & 0.49914800 \\ -9.03658000 & 2.62080200 & 1.18428200 \\ -10.24385000 & 3.19983900 & 1.61668700 \\ -10.20336100 & -0.60257300 & -0.29739500 \\ -8.97495100 & -1.30238300 & -0.52024000 \\ -7.72656800 & -0.66613900 & -0.10826500\end{array}$




\begin{tabular}{|c|c|c|c|}
\hline $\mathrm{C}$ & -7.72192300 & 0.75667900 & 0.10020400 \\
\hline $\mathrm{C}$ & -11.43924200 & -1.22966300 & -0.63878200 \\
\hline $\mathrm{C}$ & -11.41816900 & -2.46551300 & -1.29200300 \\
\hline $\mathrm{C}$ & -10.21936500 & -3.11218300 & -1.63720900 \\
\hline $\mathrm{C}$ & -9.02099200 & -2.52420100 & -1.22259900 \\
\hline $\mathrm{C}$ & -12.67386800 & 0.63102100 & 0.32624300 \\
\hline $\mathrm{C}$ & -12.67297900 & -0.57143600 & -0.30745700 \\
\hline $\mathrm{C}$ & -6.50829100 & -1.39019600 & 0.08589400 \\
\hline $\mathrm{C}$ & -5.27417400 & -0.68059900 & 0.12862500 \\
\hline $\mathrm{C}$ & -5.26865200 & 0.73453400 & -0.13335700 \\
\hline $\mathrm{C}$ & -6.49910700 & 1.45994000 & -0.08931500 \\
\hline $\mathrm{C}$ & -4.03412200 & -1.33865500 & 0.39011900 \\
\hline $\mathrm{C}$ & -2.83612200 & -0.67918900 & 0.22201200 \\
\hline $\mathrm{C}$ & -2.83080100 & 0.70493500 & -0.22681500 \\
\hline $\mathrm{C}$ & -4.02415500 & 1.37883600 & -0.38915200 \\
\hline $\mathrm{C}$ & 0.71407800 & 2.26925200 & -0.75811200 \\
\hline $\mathrm{C}$ & -0.70732700 & 2.27554700 & -0.75739000 \\
\hline $\mathrm{C}$ & -1.43803400 & 1.13457100 & -0.37841000 \\
\hline $\mathrm{C}$ & -0.68731500 & 0.00317500 & -0.00228300 \\
\hline $\mathrm{C}$ & 0.67812100 & -0.00197200 & -0.00299700 \\
\hline $\mathrm{C}$ & 1.43572700 & 1.12227900 & -0.37983400 \\
\hline $\mathrm{C}$ & -1.44490900 & -1.12126400 & 0.37430700 \\
\hline $\mathrm{C}$ & -0.72319100 & -2.26836800 & 0.75264200 \\
\hline $\mathrm{C}$ & 0.69802000 & -2.27456300 & 0.75217000 \\
\hline $\mathrm{C}$ & 1.42882800 & -1.13341600 & 0.37329900 \\
\hline $\mathrm{C}$ & 2.82712800 & 0.67984500 & -0.22759400 \\
\hline $\mathrm{C}$ & 2.82141900 & -0.70411600 & 0.22190700 \\
\hline $\mathrm{C}$ & 4.01466800 & -1.37835600 & 0.38520100 \\
\hline $\mathrm{C}$ & 5.25915900 & -0.73479700 & 0.12885500 \\
\hline $\mathrm{C}$ & 5.26535100 & 0.68002000 & -0.13541300 \\
\hline $\mathrm{C}$ & 4.02515500 & 1.33849500 & -0.39683000 \\
\hline $\mathrm{C}$ & 6.48930200 & -1.46088000 & 0.08562300 \\
\hline $\mathrm{C}$ & 7.71183000 & -0.75921700 & -0.10867100 \\
\hline $\mathrm{C}$ & 7.71829400 & 0.66393500 & 0.09662900 \\
\hline $\mathrm{C}$ & 6.49974400 & 1.38879900 & -0.09562000 \\
\hline $\mathrm{C}$ & 8.96738500 & -1.40603400 & -0.50895300 \\
\hline $\mathrm{C}$ & 10.19513400 & -0.69869100 & -0.29703400 \\
\hline $\mathrm{C}$ & 10.19533700 & 0.59706400 & 0.28164400 \\
\hline $\mathrm{C}$ & 8.96821500 & 1.29920900 & 0.50481800 \\
\hline $\mathrm{C}$ & 11.43259500 & 1.22305500 & 0.61955600 \\
\hline $\mathrm{C}$ & 11.41441600 & 2.46047200 & 1.26998500 \\
\hline $\mathrm{C}$ & 10.21710500 & 3.10989200 & 1.61567700 \\
\hline $\mathrm{C}$ & 9.01731100 & 2.52284800 & 1.20440700 \\
\hline $\mathrm{C}$ & 9.02136800 & -2.62642800 & -1.19288900 \\
\hline
\end{tabular}




\begin{tabular}{|c|c|c|c|}
\hline $\mathrm{C}$ & 10.22695100 & -3.20748700 & -1.62812300 \\
\hline $\mathrm{C}$ & 11.41930200 & -2.55151100 & -1.31228600 \\
\hline $\mathrm{C}$ & 11.43234000 & -1.31295700 & -0.65555600 \\
\hline $\mathrm{C}$ & 12.66307400 & -0.64138100 & -0.34389300 \\
\hline $\mathrm{C}$ & 12.66484100 & 0.56233800 & 0.28763100 \\
\hline $\mathrm{C}$ & -6.47596000 & 2.92281800 & -0.35321900 \\
\hline $\mathrm{C}$ & -10.21636300 & 4.51609200 & 2.41317300 \\
\hline $\mathrm{C}$ & -11.62477600 & 4.97188200 & 2.84025700 \\
\hline $\mathrm{C}$ & -9.58964400 & 5.62994600 & 1.54053300 \\
\hline $\mathrm{C}$ & -9.36442600 & 4.32253800 & 3.69143200 \\
\hline $\mathrm{C}$ & -10.27036400 & -4.42590200 & -2.43582500 \\
\hline $\mathrm{C}$ & -8.86865200 & -4.95024700 & -2.79818000 \\
\hline $\mathrm{C}$ & -11.05348800 & -4.19859400 & -3.75212200 \\
\hline $\mathrm{C}$ & -10.99088400 & -5.50233200 & -1.58709700 \\
\hline $\mathrm{C}$ & -6.52084200 & -2.84962900 & 0.34948400 \\
\hline $\mathrm{C}$ & 6.51282200 & 2.84790800 & -0.36072500 \\
\hline $\mathrm{C}$ & 6.46774500 & -2.92239600 & 0.35740600 \\
\hline $\mathrm{C}$ & 10.27157300 & 4.42526700 & 2.41134000 \\
\hline $\mathrm{C}$ & 8.87128700 & 4.95275100 & 2.77460600 \\
\hline $\mathrm{C}$ & 10.99267000 & 5.49861800 & 1.55922700 \\
\hline $\mathrm{C}$ & 11.05625600 & 4.19943000 & 3.72696400 \\
\hline $\mathrm{C}$ & 10.19495900 & -4.52147500 & -2.42828000 \\
\hline $\mathrm{C}$ & 9.55642500 & -5.63495900 & -1.56379500 \\
\hline $\mathrm{C}$ & 11.60261700 & -4.98495900 & -2.84950600 \\
\hline $\mathrm{C}$ & 9.35104000 & -4.31706300 & -3.71024900 \\
\hline $\mathrm{C}$ & -5.69318400 & 3.81443800 & 0.40570700 \\
\hline $\mathrm{C}$ & -5.64367900 & 5.16794400 & 0.08818100 \\
\hline $\mathrm{C}$ & -6.34821000 & 5.70014600 & -1.00820300 \\
\hline $\mathrm{C}$ & -7.12988300 & 4.80343100 & -1.75445000 \\
\hline $\mathrm{C}$ & -7.20408000 & 3.45023800 & -1.43156300 \\
\hline $\mathrm{C}$ & -5.73103400 & -3.75624600 & -0.37926900 \\
\hline $\mathrm{C}$ & -5.74395300 & -5.11951200 & -0.08469000 \\
\hline $\mathrm{C}$ & -6.52191000 & -5.63997400 & 0.96147700 \\
\hline $\mathrm{C}$ & -7.30424100 & -4.72192500 & 1.69055100 \\
\hline $\mathrm{C}$ & -7.31828400 & -3.36744500 & 1.38973800 \\
\hline $\mathrm{C}$ & 5.69187000 & -3.81922900 & -0.39562500 \\
\hline $\mathrm{C}$ & 5.64699400 & -5.17675900 & -0.07446700 \\
\hline $\mathrm{C}$ & 6.35047200 & -5.69759000 & 1.02232700 \\
\hline $\mathrm{C}$ & 7.12727100 & -4.78956500 & 1.76840800 \\
\hline $\mathrm{C}$ & 7.19857900 & -3.44160300 & 1.44248200 \\
\hline $\mathrm{C}$ & 5.72458700 & 3.75560200 & 0.36842600 \\
\hline $\mathrm{C}$ & 5.73755600 & 5.11854400 & 0.07242300 \\
\hline $\mathrm{C}$ & 6.51396500 & 5.63761400 & -0.97560600 \\
\hline $\mathrm{C}$ & 7.29475700 & 4.71852100 & -1.70501800 \\
\hline
\end{tabular}




\begin{tabular}{|c|c|c|c|}
\hline $\mathrm{C}$ & 7.30879000 & 3.36434600 & -1.40282600 \\
\hline $\mathrm{C}$ & -6.23160700 & 7.19466100 & -1.35040300 \\
\hline $\mathrm{C}$ & -4.75442500 & 7.52002700 & -1.68276400 \\
\hline $\mathrm{C}$ & -6.68123700 & 8.04355400 & -0.13675300 \\
\hline $\mathrm{C}$ & -7.09845200 & 7.58686800 & -2.56181300 \\
\hline $\mathrm{C}$ & -6.54408900 & -7.13217300 & 1.32707300 \\
\hline $\mathrm{C}$ & -7.99238400 & -7.66762200 & 1.21329300 \\
\hline $\mathrm{C}$ & -6.04746800 & -7.30366900 & 2.78401600 \\
\hline $\mathrm{C}$ & -5.64283000 & -7.97481100 & 0.40503000 \\
\hline $\mathrm{C}$ & 6.53605100 & 7.12941400 & -1.34283000 \\
\hline $\mathrm{C}$ & 7.98471700 & 7.66448300 & -1.23200000 \\
\hline $\mathrm{C}$ & 5.63659500 & 7.97334600 & -0.42021000 \\
\hline $\mathrm{C}$ & 6.03709400 & 7.29950400 & -2.79914000 \\
\hline $\mathrm{C}$ & 6.29031700 & -7.17841400 & 1.43065600 \\
\hline $\mathrm{C}$ & 5.68439700 & -7.28273400 & 2.85220300 \\
\hline $\mathrm{C}$ & 7.71861600 & -7.77472900 & 1.43894400 \\
\hline $\mathrm{C}$ & 5.42339200 & -8.01528800 & 0.47116100 \\
\hline $\mathrm{H}$ & -12.38925500 & 2.97320200 & 1.57728900 \\
\hline $\mathrm{H}$ & -8.11369400 & 3.13155300 & 1.41777500 \\
\hline $\mathrm{H}$ & -12.37018900 & -2.91679600 & -1.55583200 \\
\hline $\mathrm{H}$ & -8.09186700 & -3.01025300 & -1.47444700 \\
\hline $\mathrm{H}$ & -13.60920600 & 1.11409800 & 0.59460500 \\
\hline $\mathrm{H}$ & -13.60681000 & -1.06450500 & -0.56244900 \\
\hline $\mathrm{H}$ & -4.04366600 & -2.37183600 & 0.71492400 \\
\hline $\mathrm{H}$ & -4.02107300 & 2.41558100 & -0.70225000 \\
\hline $\mathrm{H}$ & 1.22696800 & 3.17687700 & -1.06354000 \\
\hline $\mathrm{H}$ & -1.21286200 & 3.18742800 & -1.06230800 \\
\hline $\mathrm{H}$ & -1.23616300 & -3.17601400 & 1.05786400 \\
\hline $\mathrm{H}$ & 1.20363700 & -3.18639600 & 1.05706800 \\
\hline $\mathrm{H}$ & 4.01150300 & -2.41489100 & 0.69900000 \\
\hline $\mathrm{H}$ & 4.03506100 & 2.37127400 & -0.72290800 \\
\hline $\mathrm{H}$ & 12.36754000 & 2.91092200 & 1.53126000 \\
\hline $\mathrm{H}$ & 8.08931300 & 3.01071600 & 1.45685700 \\
\hline $\mathrm{H}$ & 8.09707600 & -3.13568300 & -1.42407800 \\
\hline $\mathrm{H}$ & 12.37275900 & -2.98457000 & -1.59262200 \\
\hline $\mathrm{H}$ & 13.59737400 & -1.12610900 & -0.61290000 \\
\hline $\mathrm{H}$ & 13.59979600 & 1.05455900 & 0.54013800 \\
\hline $\mathrm{H}$ & -12.27125500 & 5.17327900 & 1.97869800 \\
\hline $\mathrm{H}$ & -11.54889300 & 5.90136800 & 3.41374000 \\
\hline $\mathrm{H}$ & -12.11972500 & 4.23207900 & 3.47936300 \\
\hline $\mathrm{H}$ & -10.17708400 & 5.79287300 & 0.62984700 \\
\hline $\mathrm{H}$ & -9.56410700 & 6.57208000 & 2.10039000 \\
\hline $\mathrm{H}$ & -8.56439400 & 5.38988600 & 1.24247200 \\
\hline $\mathrm{H}$ & -9.78599500 & 3.54004300 & 4.33210700 \\
\hline
\end{tabular}




\begin{tabular}{|c|c|c|c|}
\hline $\mathrm{H}$ & -9.33937300 & 5.25410100 & 4.26792400 \\
\hline $\mathrm{H}$ & -8.32923200 & 4.04896800 & 3.46093000 \\
\hline $\mathrm{H}$ & -8.31121800 & -4.23390100 & -3.41316000 \\
\hline $\mathrm{H}$ & -8.27147900 & -5.18106000 & -1.90918100 \\
\hline $\mathrm{H}$ & -8.96482500 & -5.87366200 & -3.37876600 \\
\hline $\mathrm{H}$ & -10.56791000 & -3.43879800 & -4.37471900 \\
\hline $\mathrm{H}$ & -12.08428100 & -3.87724900 & -3.57196300 \\
\hline $\mathrm{H}$ & -11.09598500 & -5.13057100 & -4.32666500 \\
\hline $\mathrm{H}$ & -12.01355200 & -5.20734500 & -1.32981800 \\
\hline $\mathrm{H}$ & -11.04963600 & -6.44310200 & -2.14618800 \\
\hline $\mathrm{H}$ & -10.45082500 & -5.69383000 & -0.65287400 \\
\hline $\mathrm{H}$ & 8.31360100 & 4.23874900 & 3.39208000 \\
\hline $\mathrm{H}$ & 8.96985400 & 5.87733900 & 3.35291800 \\
\hline $\mathrm{H}$ & 8.27314600 & 5.18248300 & 1.88597800 \\
\hline $\mathrm{H}$ & 10.45150200 & 5.68912100 & 0.62544300 \\
\hline $\mathrm{H}$ & 12.01443100 & 5.20132300 & 1.30098700 \\
\hline $\mathrm{H}$ & 11.05395100 & 6.44045600 & 2.11623900 \\
\hline $\mathrm{H}$ & 10.57029100 & 3.44181800 & 4.35191700 \\
\hline $\mathrm{H}$ & 11.10122400 & 5.13256300 & 4.29943600 \\
\hline $\mathrm{H}$ & 12.08623500 & 3.87592600 & 3.54598200 \\
\hline $\mathrm{H}$ & 10.13647800 & -5.80453800 & -0.64978700 \\
\hline $\mathrm{H}$ & 9.53026200 & -6.57487400 & -2.12745700 \\
\hline $\mathrm{H}$ & 8.53027800 & -5.39119400 & -1.27155300 \\
\hline $\mathrm{H}$ & 12.10481200 & -4.24740400 & -3.48553100 \\
\hline $\mathrm{H}$ & 12.24386000 & -5.19095500 & -1.98511000 \\
\hline $\mathrm{H}$ & 11.52400400 & -5.91327400 & -3.42451500 \\
\hline $\mathrm{H}$ & 9.78177400 & -3.53558500 & -4.34604800 \\
\hline $\mathrm{H}$ & 8.31693200 & -4.03608000 & -3.48369500 \\
\hline $\mathrm{H}$ & 9.32171800 & -5.24639200 & -4.29018200 \\
\hline $\mathrm{H}$ & -5.14213500 & 3.44656900 & 1.26729600 \\
\hline $\mathrm{H}$ & -5.03949100 & 5.82199400 & 0.70976400 \\
\hline $\mathrm{H}$ & -7.69262500 & 5.15621000 & -2.61083600 \\
\hline $\mathrm{H}$ & -7.81520400 & 2.78956600 & -2.03935200 \\
\hline $\mathrm{H}$ & -5.13370600 & -3.39855200 & -1.21359000 \\
\hline $\mathrm{H}$ & -5.13672900 & -5.78099200 & -0.69181700 \\
\hline $\mathrm{H}$ & -7.91669800 & -5.07273200 & 2.51538700 \\
\hline $\mathrm{H}$ & -7.93357500 & -2.69360300 & 1.97811200 \\
\hline $\mathrm{H}$ & 5.14010300 & -3.45852600 & -1.25985400 \\
\hline $\mathrm{H}$ & 5.04819800 & -5.83127400 & -0.69744800 \\
\hline $\mathrm{H}$ & 7.68758700 & -5.14123500 & 2.62925900 \\
\hline $\mathrm{H}$ & 7.80573200 & -2.77461300 & 2.04728600 \\
\hline $\mathrm{H}$ & 5.12841000 & 3.39893400 & 1.20400500 \\
\hline $\mathrm{H}$ & 5.13160300 & 5.78090100 & 0.67985900 \\
\hline $\mathrm{H}$ & 7.90596900 & 5.06824500 & -2.53123700 \\
\hline
\end{tabular}




\begin{tabular}{lrrr}
$\mathrm{H}$ & 7.92277600 & 2.68965300 & -1.99158600 \\
$\mathrm{H}$ & -4.08845800 & 7.30614500 & -0.83965600 \\
$\mathrm{H}$ & -4.40712500 & 6.93974800 & -2.54531700 \\
$\mathrm{H}$ & -4.65002500 & 8.58323800 & -1.92662800 \\
$\mathrm{H}$ & -7.72783800 & 7.84426800 & 0.11828200 \\
$\mathrm{H}$ & -6.58802000 & 9.10978900 & -0.37140900 \\
$\mathrm{H}$ & -6.07382800 & 7.84727900 & 0.75304100 \\
$\mathrm{H}$ & -8.16271700 & 7.39742700 & -2.38218200 \\
$\mathrm{H}$ & -6.98586200 & 8.65747300 & -2.76115400 \\
$\mathrm{H}$ & -6.80106100 & 7.05231700 & -3.47109800 \\
$\mathrm{H}$ & -8.68024700 & -7.14131100 & 1.88311900 \\
$\mathrm{H}$ & -8.02075000 & -8.73004200 & 1.47966500 \\
$\mathrm{H}$ & -8.37180200 & -7.56597000 & 0.19017300 \\
$\mathrm{H}$ & -5.01977000 & -6.93981800 & 2.89655600 \\
$\mathrm{H}$ & -6.67630600 & -6.76368600 & 3.49936300 \\
$\mathrm{H}$ & -6.06365800 & -8.36327400 & 3.06240500 \\
$\mathrm{H}$ & -5.95876600 & -7.91710600 & -0.64284700 \\
$\mathrm{H}$ & -4.59194000 & -7.66911500 & 0.46674300 \\
$\mathrm{H}$ & -5.69474300 & -9.02653500 & 0.70441200 \\
$\mathrm{H}$ & 8.36576900 & 7.56383700 & -0.20938800 \\
$\mathrm{H}$ & 8.01301400 & 8.72659800 & -1.49959100 \\
$\mathrm{H}$ & 8.67130200 & 7.13719800 & -1.90237100 \\
$\mathrm{H}$ & 5.17276100 & -7.72646800 & 0.44307600 \\
$\mathrm{H}$ & 5.38233100 & -7.67243500 & 0.45257600 \\
$\mathrm{H}$ & 5.95422900 & 7.91664900 & 0.62720800 \\
$\mathrm{H}$ & 5.68838700 & 9.02473100 & -0.72080200 \\
$\mathrm{H}$ & 4.58550000 & 7.66795400 & -0.47988400 \\
$\mathrm{H}$ & 5.00909900 & 6.93585300 & -2.90960500 \\
$\mathrm{H}$ & 6.66458900 & 6.75856800 & -3.51494400 \\
$\mathrm{H}$ & 6.05315100 & 8.35880700 & -3.07868000 \\
$\mathrm{H}$ & 4.66722900 & -6.87515100 & 2.87916600 \\
$\mathrm{H}$ & 6.28289300 & -6.74344800 & 3.59376300 \\
$\mathrm{H}$ & 5.63676400 & -8.33221200 & 3.16377800 \\
$\mathrm{H}$ & 8.38018900 & -7.25191300 & 2.13723900 \\
$\mathrm{H}$ & 7.68264200 & -8.82658200 & 1.74374600 \\
$\mathrm{H}$ & -7.99720500 & -0.55366900 \\
$\mathrm{H}$ & -9.05947000 & 0.80014100 \\
\hline
\end{tabular}

\section{OT $6^{2 \cdot+}$}

Energy (with Zero Point Energy correction $)=-4408.933375$ (-4407.041380) Hartree Free energy $(298 \mathrm{~K})=-4407.186990$ Hartree

Zero number of imaginary frequency

23

$\begin{array}{llll}\mathrm{C} & 11.48544300 & 2.58644000 & -1.17055900\end{array}$ 


\begin{tabular}{|c|c|c|c|}
\hline $\mathrm{C}$ & 11.46583600 & 1.33022900 & -0.53086400 \\
\hline $\mathrm{C}$ & 10.21309200 & 0.72222800 & -0.21058400 \\
\hline $\mathrm{C}$ & 9.00128800 & 1.43142400 & -0.44723100 \\
\hline $\mathrm{C}$ & 9.08596800 & 2.66009900 & -1.10522200 \\
\hline $\mathrm{C}$ & 10.31251200 & 3.25142400 & -1.49710100 \\
\hline $\mathrm{C}$ & 10.19134000 & -0.58074700 & 0.35504900 \\
\hline $\mathrm{C}$ & 8.95824400 & -1.28075300 & 0.52769100 \\
\hline $\mathrm{C}$ & 7.72632100 & -0.65519000 & 0.08536000 \\
\hline $\mathrm{C}$ & 7.72158000 & 0.78109300 & -0.09456300 \\
\hline $\mathrm{C}$ & 11.41099900 & -1.21908300 & 0.71316000 \\
\hline $\mathrm{C}$ & 11.37133000 & -2.47211600 & 1.34888900 \\
\hline $\mathrm{C}$ & 10.16330500 & -3.12259500 & 1.66129800 \\
\hline $\mathrm{C}$ & 8.98453400 & -2.52239700 & 1.22699200 \\
\hline $\mathrm{C}$ & 12.67496300 & 0.64822800 & -0.19722700 \\
\hline $\mathrm{C}$ & 12.65231400 & -0.57148500 & 0.42156600 \\
\hline $\mathrm{C}$ & 6.51192600 & -1.39333100 & -0.11142200 \\
\hline $\mathrm{C}$ & 5.27811500 & -0.69324500 & -0.13647700 \\
\hline $\mathrm{C}$ & 5.27414300 & 0.72338000 & 0.13099500 \\
\hline $\mathrm{C}$ & 6.50557700 & 1.46308300 & 0.08437900 \\
\hline $\mathrm{C}$ & 4.03180600 & -1.35466600 & -0.39258400 \\
\hline $\mathrm{C}$ & 2.84118800 & -0.69689000 & -0.22009600 \\
\hline $\mathrm{C}$ & 2.84019400 & 0.69237700 & 0.21948600 \\
\hline $\mathrm{C}$ & 4.03118400 & 1.37010700 & 0.37244400 \\
\hline $\mathrm{C}$ & -0.70559200 & 2.27219200 & 0.74321200 \\
\hline $\mathrm{C}$ & 0.72510700 & 2.27069800 & 0.74183400 \\
\hline $\mathrm{C}$ & 1.44300200 & 1.13001300 & 0.36906300 \\
\hline $\mathrm{C}$ & 0.68837300 & -0.00138000 & -0.00067100 \\
\hline $\mathrm{C}$ & -0.67649800 & 0.00138700 & 0.00014500 \\
\hline $\mathrm{C}$ & -1.42678100 & 1.13426300 & 0.37131500 \\
\hline $\mathrm{C}$ & 1.43864800 & -1.13437400 & -0.37153100 \\
\hline $\mathrm{C}$ & 0.71746500 & -2.27232900 & -0.74331800 \\
\hline $\mathrm{C}$ & -0.71322100 & -2.27083200 & -0.74182500 \\
\hline $\mathrm{C}$ & -1.43115100 & -1.13013900 & -0.36911500 \\
\hline $\mathrm{C}$ & -2.82928800 & 0.69648200 & 0.22067200 \\
\hline $\mathrm{C}$ & -2.82829500 & -0.69275100 & -0.21873600 \\
\hline $\mathrm{C}$ & -4.01934600 & -1.37086200 & -0.37056600 \\
\hline $\mathrm{C}$ & -5.26202900 & -0.72457500 & -0.12739000 \\
\hline $\mathrm{C}$ & -5.26637100 & 0.69211300 & 0.14026900 \\
\hline $\mathrm{C}$ & -4.02000800 & 1.35400400 & 0.39431600 \\
\hline $\mathrm{C}$ & -6.49322000 & -1.46434600 & -0.07875700 \\
\hline $\mathrm{C}$ & -7.70845800 & -0.78267500 & 0.10686700 \\
\hline $\mathrm{C}$ & -7.71554100 & 0.65308500 & -0.07380200 \\
\hline $\mathrm{C}$ & -6.50063500 & 1.39188000 & 0.11782700 \\
\hline $\mathrm{C}$ & -8.98503200 & -1.43441300 & 0.46666200 \\
\hline
\end{tabular}




\begin{tabular}{|c|c|c|c|}
\hline $\mathrm{C}$ & -10.19908500 & -0.72882500 & 0.23153300 \\
\hline $\mathrm{C}$ & -10.18148000 & 0.57330000 & -0.33616300 \\
\hline $\mathrm{C}$ & -8.95027400 & 1.27625100 & -0.51224600 \\
\hline $\mathrm{C}$ & -11.40362500 & 1.20808200 & -0.69211100 \\
\hline $\mathrm{C}$ & -11.36856700 & 2.46079500 & -1.32848600 \\
\hline $\mathrm{C}$ & -10.16287700 & 3.11450600 & -1.64328600 \\
\hline $\mathrm{C}$ & -8.98145700 & 2.51763300 & -1.21144600 \\
\hline $\mathrm{C}$ & -9.06381400 & -2.65974900 & 1.13144200 \\
\hline $\mathrm{C}$ & -10.28748700 & -3.25205500 & 1.53052500 \\
\hline $\mathrm{C}$ & -11.46334200 & -2.59276900 & 1.20214100 \\
\hline $\mathrm{C}$ & -11.44923100 & -1.33932800 & 0.55686400 \\
\hline $\mathrm{C}$ & -12.66094300 & -0.66132700 & 0.22387300 \\
\hline $\mathrm{C}$ & -12.64264900 & 0.55720800 & -0.39719600 \\
\hline $\mathrm{C}$ & 6.44296400 & 2.93772300 & 0.31646000 \\
\hline $\mathrm{C}$ & 10.29962400 & 4.59034100 & -2.25375800 \\
\hline $\mathrm{C}$ & 11.71523800 & 5.04522300 & -2.65631200 \\
\hline $\mathrm{C}$ & 9.67618800 & 5.68027600 & -1.34944900 \\
\hline $\mathrm{C}$ & 9.45529300 & 4.44354100 & -3.54310200 \\
\hline $\mathrm{C}$ & 10.19497200 & -4.44319500 & 2.44963800 \\
\hline $\mathrm{C}$ & 8.78339200 & -4.96173800 & 2.77894600 \\
\hline $\mathrm{C}$ & 10.95326100 & -4.22896100 & 3.78240100 \\
\hline $\mathrm{C}$ & 10.92764500 & -5.51389100 & 1.60471400 \\
\hline $\mathrm{C}$ & 6.54753200 & -2.84982500 & -0.38853900 \\
\hline $\mathrm{C}$ & -6.53564900 & 2.84869000 & 0.39291900 \\
\hline $\mathrm{C}$ & -6.43609600 & -2.93766900 & -0.31932200 \\
\hline $\mathrm{C}$ & -10.19971000 & 4.43508300 & -2.43145100 \\
\hline $\mathrm{C}$ & -8.79022100 & 4.95759500 & -2.76346300 \\
\hline $\mathrm{C}$ & -10.93367000 & 5.50372200 & -1.58503100 \\
\hline $\mathrm{C}$ & -10.95999100 & 4.21889900 & -3.76276700 \\
\hline $\mathrm{C}$ & -10.26816300 & -4.58237900 & 2.30224100 \\
\hline $\mathrm{C}$ & -9.61957400 & -5.67707700 & 1.42180800 \\
\hline $\mathrm{C}$ & -11.68323400 & -5.04966700 & 2.69220600 \\
\hline $\mathrm{C}$ & -9.44244500 & -4.40737600 & 3.60026600 \\
\hline $\mathrm{C}$ & 5.73269600 & 3.80051700 & -0.53672400 \\
\hline $\mathrm{C}$ & 5.63286500 & 5.16031200 & -0.25430500 \\
\hline $\mathrm{C}$ & 6.22006100 & 5.72557200 & 0.89275900 \\
\hline $\mathrm{C}$ & 6.93248700 & 4.85617100 & 1.73197200 \\
\hline $\mathrm{C}$ & 7.04684500 & 3.49393300 & 1.45127100 \\
\hline $\mathrm{C}$ & 5.75797000 & -3.77741500 & 0.31466000 \\
\hline $\mathrm{C}$ & 5.78828400 & -5.13452800 & -0.00253600 \\
\hline $\mathrm{C}$ & 6.58721800 & -5.63147900 & -1.04596700 \\
\hline $\mathrm{C}$ & 7.37329900 & -4.69573100 & -1.74581800 \\
\hline $\mathrm{C}$ & 7.36726800 & -3.34528400 & -1.42367800 \\
\hline $\mathrm{C}$ & -5.72874300 & -3.80863800 & 0.52163900 \\
\hline
\end{tabular}




\begin{tabular}{|c|c|c|c|}
\hline $\mathrm{C}$ & -5.64082000 & -5.17293800 & 0.23441800 \\
\hline $\mathrm{C}$ & -6.23848400 & -5.72455600 & -0.90846400 \\
\hline $\mathrm{C}$ & -6.94823900 & -4.84085900 & -1.74233400 \\
\hline $\mathrm{C}$ & -7.05248000 & -3.48354500 & -1.45716200 \\
\hline $\mathrm{C}$ & -5.74863300 & 3.77518700 & -0.31454700 \\
\hline $\mathrm{C}$ & -5.77850400 & 5.13290500 & 0.00011600 \\
\hline $\mathrm{C}$ & -6.57427700 & 5.63151000 & 1.04515100 \\
\hline $\mathrm{C}$ & -7.35766600 & 4.69677900 & 1.74940800 \\
\hline $\mathrm{C}$ & -7.35220400 & 3.34574300 & 1.42980200 \\
\hline $\mathrm{C}$ & 6.04828800 & 7.22550400 & 1.19073300 \\
\hline $\mathrm{C}$ & 4.54239000 & 7.53075900 & 1.38143300 \\
\hline $\mathrm{C}$ & 6.59212300 & 8.06008200 & 0.00670100 \\
\hline $\mathrm{C}$ & 6.79469400 & 7.65704200 & 2.46723300 \\
\hline $\mathrm{C}$ & 6.62399900 & -7.11684100 & -1.43794900 \\
\hline $\mathrm{C}$ & 8.07343300 & -7.64634300 & -1.31254400 \\
\hline $\mathrm{C}$ & 6.14992600 & -7.26419400 & -2.90501600 \\
\hline $\mathrm{C}$ & 5.71407000 & -7.98143600 & -0.54511900 \\
\hline $\mathrm{C}$ & -6.61053600 & 7.11762600 & 1.43431900 \\
\hline $\mathrm{C}$ & -8.06064900 & 7.64612500 & 1.31254600 \\
\hline $\mathrm{C}$ & -5.70395400 & 7.98090800 & 0.53682900 \\
\hline $\mathrm{C}$ & -6.13179300 & 7.26813800 & 2.89954600 \\
\hline $\mathrm{C}$ & -6.12999000 & -7.21461400 & -1.27612600 \\
\hline $\mathrm{C}$ & -5.39868600 & -7.34693300 & -2.63436600 \\
\hline $\mathrm{C}$ & -7.54633700 & -7.82609700 & -1.39742900 \\
\hline $\mathrm{C}$ & -5.34583600 & -8.02076500 & -0.22353200 \\
\hline $\mathrm{H}$ & 12.45088000 & 3.01344800 & -1.41670300 \\
\hline $\mathrm{H}$ & 8.17276000 & 3.18270800 & -1.34960300 \\
\hline $\mathrm{H}$ & 12.31698700 & -2.92775300 & 1.62818900 \\
\hline $\mathrm{H}$ & 8.04459100 & -2.99539200 & 1.46160800 \\
\hline $\mathrm{H}$ & 13.62190100 & 1.12513100 & -0.43415100 \\
\hline $\mathrm{H}$ & 13.57857800 & -1.07316400 & 0.68679400 \\
\hline $\mathrm{H}$ & 4.04045400 & -2.38706700 & -0.71881500 \\
\hline $\mathrm{H}$ & 4.02629500 & 2.41434500 & 0.65846500 \\
\hline $\mathrm{H}$ & -1.21162600 & 3.18515500 & 1.04449300 \\
\hline $\mathrm{H}$ & 1.23457300 & 3.18220300 & 1.04177000 \\
\hline $\mathrm{H}$ & 1.22351000 & -3.18535400 & -1.04438900 \\
\hline $\mathrm{H}$ & -1.22270200 & -3.18240400 & -1.04152700 \\
\hline $\mathrm{H}$ & -4.01481600 & -2.41521500 & -0.65622500 \\
\hline $\mathrm{H}$ & -4.02853700 & 2.38646100 & 0.72040400 \\
\hline $\mathrm{H}$ & -12.31595600 & 2.91388400 & -1.60606100 \\
\hline $\mathrm{H}$ & -8.04337400 & 2.99337300 & -1.44797300 \\
\hline $\mathrm{H}$ & -8.14799600 & -3.17772000 & 1.37624600 \\
\hline $\mathrm{H}$ & -12.42687400 & -3.02165800 & 1.45246400 \\
\hline $\mathrm{H}$ & -13.60617200 & -1.14012200 & 0.46379800 \\
\hline
\end{tabular}


$\mathrm{H}$

$\mathrm{H}$

$\mathrm{H}$

$\mathrm{H}$

$\mathrm{H}$

$\mathrm{H}$

$\mathrm{H}$

$\mathrm{H}$

$\mathrm{H}$

$\mathrm{H}$

$\mathrm{H}$

$\mathrm{H}$

$\mathrm{H}$

$\mathrm{H}$

$\mathrm{H}$

$\mathrm{H}$

$\mathrm{H}$

$\mathrm{H}$

$\mathrm{H}$

$\mathrm{H}$

$\mathrm{H}$

$\mathrm{H}$

$\mathrm{H}$

$\mathrm{H}$

$\mathrm{H}$

$\mathrm{H}$

$\mathrm{H}$

$\mathrm{H}$

$\mathrm{H}$

$\mathrm{H}$

$\mathrm{H}$

$\mathrm{H}$

$\mathrm{H}$

$\mathrm{H}$

$\mathrm{H}$

$\mathrm{H}$

$\mathrm{H}$

$\mathrm{H}$

$\mathrm{H}$

$\mathrm{H}$

$\mathrm{H}$

$\mathrm{H}$

$\mathrm{H}$

$\mathrm{H}$

$\begin{array}{rrr}-13.57076500 & 1.05598900 & -0.66143100 \\ 12.35565600 & 5.21761000 & -1.78398000 \\ 11.65256700 & 5.99068900 & -3.20462600 \\ 12.20922200 & 4.31828800 & -3.31095000 \\ 10.26357500 & 5.81432900 & -0.43405100 \\ 9.65514700 & 6.63835100 & -1.88170400 \\ 8.64967500 & 5.43646700 & -1.05908000 \\ 9.87290300 & 3.67577600 & -4.20397200 \\ 9.44576000 & 5.39212600 & -4.09144500 \\ 8.41468600 & 4.17699500 & -3.32969600 \\ 8.21839700 & -4.24784300 & 3.38995600 \\ 8.20269800 & -5.18064400 & 1.87631400 \\ 8.86254700 & -5.89084500 & 3.35272700 \\ 10.45929100 & -3.47207200 & 4.40186400 \\ 11.98971700 & -3.91247800 & 3.62599300 \\ 10.98015200 & -5.16550200 & 4.35003300 \\ 11.95627500 & -5.22064800 & 1.36872900 \\ 10.97420100 & -6.45913100 & 2.15699900 \\ 10.40416400 & -5.69661500 & 0.65947600 \\ -8.22445600 & 4.24539000 & -3.37572700 \\ -8.87308300 & 5.88660000 & -3.33689000 \\ -8.20832100 & 5.17791400 & -1.86194700 \\ -10.40878400 & 5.68791300 & -0.64085600 \\ -11.96098800 & 5.20759200 & -1.34696100 \\ -10.98397700 & 6.44884100 & -2.13719800 \\ -10.46514400 & 3.46341600 & -4.38324900 \\ -10.99055300 & 5.15541200 & -4.33026700 \\ -11.99526100 & 3.89954600 & -3.60437100 \\ -10.18938000 & -5.82773800 & 0.49808500 \\ -9.59993000 & -6.62841500 & 1.96619100 \\ -8.59010100 & -5.42851900 & 1.14609400 \\ -12.19290100 & -4.32325600 & 3.33525100 \\ -12.31133100 & -5.23590300 & 1.81374500 \\ -11.61656200 & -5.98980900 & 3.24913500 \\ -9.87997600 & -3.63825600 & 4.24649000 \\ -8.40370500 & -4.12663300 & 3.39583600 \\ -9.42502300 & -5.34905300 & 4.16027600 \\ 5.26080200 & 3.40392700 & -1.43230500 \\ 5.07798300 & 5.79064600 & -0.94308800 \\ 7.40562400 & 5.23411300 & 2.63093900 \\ 7.59934300 & 2.85336400 & 2.13303300 \\ 5.14125600 & -3.43915300 & 1.14270200 \\ 5.17688900 & -5.81158000 & 0.58285900 \\ 8.00266300 & -5.02717500 & -2.56596400\end{array}$




\begin{tabular}{|c|c|c|c|}
\hline $\mathrm{H}$ & 7.98060400 & -2.65560000 & -1.99572200 \\
\hline $\mathrm{H}$ & -5.24836900 & -3.42138500 & 1.41685700 \\
\hline $\mathrm{H}$ & -5.08935100 & -5.80607200 & 0.92024000 \\
\hline $\mathrm{H}$ & -7.42652500 & -5.21605600 & -2.64213500 \\
\hline $\mathrm{H}$ & -7.60379100 & -2.83426800 & -2.13153600 \\
\hline $\mathrm{H}$ & -5.13439200 & 3.43551200 & -1.14384600 \\
\hline $\mathrm{H}$ & -5.16929500 & 5.80908300 & -0.58855500 \\
\hline $\mathrm{H}$ & -7.98446000 & 5.02956000 & 2.57097900 \\
\hline $\mathrm{H}$ & -7.96344800 & 2.65690800 & 2.00509300 \\
\hline $\mathrm{H}$ & 3.95977200 & 7.28757800 & 0.48617500 \\
\hline $\mathrm{H}$ & 4.12799100 & 6.96011200 & 2.22056800 \\
\hline $\mathrm{H}$ & 4.39739600 & 8.59631200 & 1.59282000 \\
\hline $\mathrm{H}$ & 7.66157600 & 7.87780800 & -0.14612800 \\
\hline $\mathrm{H}$ & 6.45754600 & 9.12887500 & 0.20783200 \\
\hline $\mathrm{H}$ & 6.07412900 & 7.83322800 & -0.93110400 \\
\hline $\mathrm{H}$ & 7.87342600 & 7.48009500 & 2.38987500 \\
\hline $\mathrm{H}$ & 6.64963300 & 8.72977500 & 2.63188800 \\
\hline $\mathrm{H}$ & 6.42284600 & 7.13666800 & 3.35714900 \\
\hline $\mathrm{H}$ & 8.76826600 & -7.10323500 & -1.96151600 \\
\hline $\mathrm{H}$ & 8.11220600 & -8.70316500 & -1.59905400 \\
\hline $\mathrm{H}$ & 8.43661700 & -7.56306700 & -0.28177900 \\
\hline $\mathrm{H}$ & 5.12238200 & -6.90301200 & -3.02626000 \\
\hline $\mathrm{H}$ & 6.78667500 & -6.70825100 & -3.60087500 \\
\hline $\mathrm{H}$ & 6.17542200 & -8.31850100 & -3.20221000 \\
\hline $\mathrm{H}$ & 6.01375900 & -7.94139100 & 0.50838500 \\
\hline $\mathrm{H}$ & 4.66250600 & -7.68068600 & -0.61714500 \\
\hline $\mathrm{H}$ & 5.77675700 & -9.02712500 & -0.86300500 \\
\hline $\mathrm{H}$ & -8.42714000 & 7.56055600 & 0.28314100 \\
\hline $\mathrm{H}$ & -8.09904000 & 8.70350800 & 1.59703100 \\
\hline $\mathrm{H}$ & -8.75308300 & 7.10397700 & 1.96487600 \\
\hline $\mathrm{H}$ & -6.00703900 & 7.93860600 & -0.51561400 \\
\hline $\mathrm{H}$ & -5.76615700 & 9.02719700 & 0.85283600 \\
\hline $\mathrm{H}$ & -4.65200500 & 7.68084100 & 0.60604400 \\
\hline $\mathrm{H}$ & -5.10365600 & 6.90776600 & 3.01816200 \\
\hline $\mathrm{H}$ & -6.76596800 & 6.71322300 & 3.59857000 \\
\hline $\mathrm{H}$ & -6.15691200 & 8.32301600 & 3.19473700 \\
\hline $\mathrm{H}$ & -4.38640000 & -6.92990500 & -2.57985900 \\
\hline $\mathrm{H}$ & -5.93221400 & -6.83009900 & -3.43892500 \\
\hline $\mathrm{H}$ & -5.31512000 & -8.40270600 & -2.91619400 \\
\hline $\mathrm{H}$ & -8.14747500 & -7.32464100 & -2.16292000 \\
\hline $\mathrm{H}$ & -7.47598800 & -8.88414600 & -1.67398100 \\
\hline $\mathrm{H}$ & -8.08767300 & -7.76054300 & -0.44705400 \\
\hline $\mathrm{H}$ & -4.31358100 & -7.66669900 & -0.11987800 \\
\hline $\mathrm{H}$ & -5.82396800 & -7.98239400 & 0.76192600 \\
\hline
\end{tabular}


$\mathrm{H}$ $\begin{array}{lll}-5.30155100 & -9.07234200 & -0.52535400\end{array}$

Mulliken spin densities:

$\begin{array}{rlc}1 & \mathrm{C} & -0.011541 \\ 2 & \mathrm{C} & 0.041675 \\ 3 & \mathrm{C} & 0.032627 \\ 4 & \mathrm{C} & -0.014547 \\ 5 & \mathrm{C} & 0.046570 \\ 6 & \mathrm{C} & 0.017345 \\ 7 & \mathrm{C} & 0.009100 \\ 8 & \mathrm{C} & 0.083462 \\ 9 & \mathrm{C} & 0.063536 \\ 10 & \mathrm{C} & -0.013000 \\ 11 & \mathrm{C} & -0.059972 \\ 12 & \mathrm{C} & 0.229471 \\ 13 & \mathrm{C} & -0.054180 \\ 14 & \mathrm{C} & 0.103394 \\ 15 & \mathrm{C} & -0.009524 \\ 16 & \mathrm{C} & 0.079505 \\ 17 & \mathrm{C} & 0.162426 \\ 18 & \mathrm{C} & -0.058355 \\ 19 & \mathrm{C} & 0.040890 \\ 20 & \mathrm{C} & 0.048669 \\ 21 & \mathrm{C} & 0.107085 \\ 22 & \mathrm{C} & -0.056197 \\ 23 & \mathrm{C} & 0.079584 \\ 24 & \mathrm{C} & 0.010543 \\ 25 & \mathrm{C} & -0.012244 \\ 26 & \mathrm{C} & 0.038553 \\ 27 & \mathrm{C} & -0.009726 \\ 28 & \mathrm{C} & -0.005443 \\ 29 & \mathrm{C} & -0.005330 \\ 30 & \mathrm{C} & 0.050546 \\ 31 & \mathrm{C} & 0.051066 \\ 32 & \mathrm{C} & -0.012788 \\ 33 & \mathrm{C} & 0.039161 \\ 34 & \mathrm{C} & -0.010201 \\ 35 & \mathrm{C} & -0.056183 \\ 36 & \mathrm{C} & 0.079773 \\ 37 & \mathrm{C} & 0.011166 \\ & \mathrm{C} & 0.040362 \\ 40.058816 \\ 39.013725\end{array}$




\begin{tabular}{|c|c|c|}
\hline 43 & $\mathrm{C}$ & 0.062749 \\
\hline 44 & $\mathrm{C}$ & 0.164084 \\
\hline 45 & $\mathrm{C}$ & -0.013857 \\
\hline 46 & $\mathrm{C}$ & 0.032705 \\
\hline 47 & $\mathrm{C}$ & 0.009394 \\
\hline 48 & $\mathrm{C}$ & 0.083539 \\
\hline 49 & $\mathrm{C}$ & -0.059780 \\
\hline 50 & $\mathrm{C}$ & 0.227761 \\
\hline 51 & $\mathrm{C}$ & -0.053275 \\
\hline 52 & $\mathrm{C}$ & 0.101794 \\
\hline 53 & $\mathrm{C}$ & 0.045023 \\
\hline 54 & $\mathrm{C}$ & 0.018057 \\
\hline 55 & $\mathrm{C}$ & -0.012280 \\
\hline 56 & $\mathrm{C}$ & 0.041601 \\
\hline 57 & $\mathrm{C}$ & -0.009974 \\
\hline 58 & $\mathrm{C}$ & 0.078884 \\
\hline 59 & $\mathrm{C}$ & -0.003134 \\
\hline 60 & $\mathrm{C}$ & -0.001046 \\
\hline 61 & $\mathrm{C}$ & -0.000049 \\
\hline 62 & $\mathrm{C}$ & 0.001095 \\
\hline 63 & $\mathrm{C}$ & 0.001020 \\
\hline 64 & $\mathrm{C}$ & 0.002553 \\
\hline 65 & $\mathrm{C}$ & 0.000014 \\
\hline 66 & $\mathrm{C}$ & -0.000028 \\
\hline 67 & $\mathrm{C}$ & 0.000274 \\
\hline 68 & $\mathrm{C}$ & 0.003084 \\
\hline 69 & $\mathrm{C}$ & 0.002827 \\
\hline 70 & $\mathrm{C}$ & -0.003200 \\
\hline 71 & $\mathrm{C}$ & 0.002506 \\
\hline 72 & $\mathrm{C}$ & 0.000016 \\
\hline 73 & $\mathrm{C}$ & 0.000292 \\
\hline 74 & $\mathrm{C}$ & -0.000011 \\
\hline 75 & $\mathrm{C}$ & -0.001083 \\
\hline 76 & $\mathrm{C}$ & 0.001081 \\
\hline 77 & $\mathrm{C}$ & -0.000050 \\
\hline 78 & $\mathrm{C}$ & 0.001069 \\
\hline 79 & $\mathrm{C}$ & 0.002915 \\
\hline 80 & $\mathrm{C}$ & -0.000771 \\
\hline 81 & $\mathrm{C}$ & 0.001981 \\
\hline 82 & $\mathrm{C}$ & 0.000353 \\
\hline 83 & $\mathrm{C}$ & 0.001323 \\
\hline 84 & $\mathrm{C}$ & 0.024670 \\
\hline 85 & $\mathrm{C}$ & -0.009027 \\
\hline 86 & $\mathrm{C}$ & 0.044426 \\
\hline
\end{tabular}




\begin{tabular}{|c|c|c|}
\hline 87 & $\mathrm{C}$ & -0.017919 \\
\hline 88 & $\mathrm{C}$ & 0.031222 \\
\hline 89 & $\mathrm{C}$ & 0.003198 \\
\hline 90 & $\mathrm{C}$ & -0.000835 \\
\hline 91 & $\mathrm{C}$ & 0.002182 \\
\hline 92 & $\mathrm{C}$ & 0.000402 \\
\hline 93 & $\mathrm{C}$ & 0.001286 \\
\hline 94 & $\mathrm{C}$ & 0.024779 \\
\hline 95 & $\mathrm{C}$ & -0.009109 \\
\hline 96 & $\mathrm{C}$ & 0.044441 \\
\hline 97 & $\mathrm{C}$ & -0.017908 \\
\hline 98 & $\mathrm{C}$ & 0.031239 \\
\hline 99 & $\mathrm{C}$ & -0.000109 \\
\hline 100 & $\mathrm{C}$ & 0.000095 \\
\hline 101 & $\mathrm{C}$ & 0.000100 \\
\hline 102 & $\mathrm{C}$ & 0.000005 \\
\hline 103 & $\mathrm{C}$ & -0.002573 \\
\hline 104 & $\mathrm{C}$ & 0.001606 \\
\hline 105 & $\mathrm{C}$ & 0.001841 \\
\hline 106 & $\mathrm{C}$ & 0.000095 \\
\hline 107 & $\mathrm{C}$ & -0.002575 \\
\hline 108 & $\mathrm{C}$ & 0.001605 \\
\hline 109 & $\mathrm{C}$ & 0.000095 \\
\hline 110 & $\mathrm{C}$ & 0.001840 \\
\hline 111 & $\mathrm{C}$ & -0.000118 \\
\hline 112 & $\mathrm{C}$ & 0.000106 \\
\hline 113 & $\mathrm{C}$ & 0.000113 \\
\hline 114 & $\mathrm{C}$ & 0.000000 \\
\hline 115 & $\mathrm{H}$ & 0.000208 \\
\hline 116 & $\mathrm{H}$ & -0.002014 \\
\hline 117 & $\mathrm{H}$ & -0.009723 \\
\hline 118 & $\mathrm{H}$ & -0.005038 \\
\hline 119 & $\mathrm{H}$ & 0.000186 \\
\hline 120 & $\mathrm{H}$ & -0.003259 \\
\hline 121 & $\mathrm{H}$ & -0.003988 \\
\hline 122 & $\mathrm{H}$ & -0.000606 \\
\hline 123 & $\mathrm{H}$ & 0.000227 \\
\hline 124 & $\mathrm{H}$ & -0.001750 \\
\hline 125 & $\mathrm{H}$ & 0.000248 \\
\hline 126 & $\mathrm{H}$ & -0.001776 \\
\hline 127 & $\mathrm{H}$ & -0.000629 \\
\hline 128 & $\mathrm{H}$ & -0.004010 \\
\hline 129 & $\mathrm{H}$ & -0.009649 \\
\hline 130 & $\mathrm{H}$ & -0.004971 \\
\hline
\end{tabular}




\begin{tabular}{|c|c|c|}
\hline 131 & $\mathrm{H}$ & -0.001953 \\
\hline 132 & $\mathrm{H}$ & 0.000240 \\
\hline 133 & $\mathrm{H}$ & 0.000205 \\
\hline 134 & $\mathrm{H}$ & -0.003234 \\
\hline 135 & $\mathrm{H}$ & 0.000031 \\
\hline 136 & $\mathrm{H}$ & -0.000029 \\
\hline 137 & $\mathrm{H}$ & 0.000034 \\
\hline 138 & $\mathrm{H}$ & -0.000015 \\
\hline 139 & $\mathrm{H}$ & 0.000591 \\
\hline 140 & $\mathrm{H}$ & -0.000034 \\
\hline 141 & $\mathrm{H}$ & -0.000016 \\
\hline 142 & $\mathrm{H}$ & 0.000590 \\
\hline 143 & $\mathrm{H}$ & -0.000023 \\
\hline 144 & $\mathrm{H}$ & 0.000049 \\
\hline 145 & $\mathrm{H}$ & -0.000060 \\
\hline 146 & $\mathrm{H}$ & -0.000026 \\
\hline 147 & $\mathrm{H}$ & -0.000037 \\
\hline 148 & $\mathrm{H}$ & 0.000083 \\
\hline 149 & $\mathrm{H}$ & 0.000591 \\
\hline 150 & $\mathrm{H}$ & 0.000063 \\
\hline 151 & $\mathrm{H}$ & 0.000683 \\
\hline 152 & $\mathrm{H}$ & -0.000105 \\
\hline 153 & $\mathrm{H}$ & 0.000050 \\
\hline 154 & $\mathrm{H}$ & -0.000026 \\
\hline 155 & $\mathrm{H}$ & -0.000060 \\
\hline 156 & $\mathrm{H}$ & -0.000104 \\
\hline 157 & $\mathrm{H}$ & 0.000062 \\
\hline 158 & $\mathrm{H}$ & 0.000689 \\
\hline 159 & $\mathrm{H}$ & -0.000036 \\
\hline 160 & $\mathrm{H}$ & 0.000595 \\
\hline 161 & $\mathrm{H}$ & 0.000082 \\
\hline 162 & $\mathrm{H}$ & -0.000013 \\
\hline 163 & $\mathrm{H}$ & 0.000585 \\
\hline 164 & $\mathrm{H}$ & -0.000032 \\
\hline 165 & $\mathrm{H}$ & 0.000033 \\
\hline 166 & $\mathrm{H}$ & 0.000032 \\
\hline 167 & $\mathrm{H}$ & -0.000030 \\
\hline 168 & $\mathrm{H}$ & -0.000017 \\
\hline 169 & $\mathrm{H}$ & -0.000026 \\
\hline 170 & $\mathrm{H}$ & 0.000601 \\
\hline 171 & $\mathrm{H}$ & -0.000168 \\
\hline 172 & $\mathrm{H}$ & 0.000182 \\
\hline 173 & $\mathrm{H}$ & 0.000078 \\
\hline 174 & $\mathrm{H}$ & -0.000008 \\
\hline
\end{tabular}




\begin{tabular}{ccc}
175 & $\mathrm{H}$ & -0.001251 \\
176 & $\mathrm{H}$ & 0.000549 \\
177 & $\mathrm{H}$ & 0.000825 \\
178 & $\mathrm{H}$ & -0.001421 \\
179 & $\mathrm{H}$ & -0.000182 \\
180 & $\mathrm{H}$ & 0.000181 \\
181 & $\mathrm{H}$ & 0.000082 \\
182 & $\mathrm{H}$ & -0.000005 \\
183 & $\mathrm{H}$ & -0.001255 \\
184 & $\mathrm{H}$ & 0.000555 \\
185 & $\mathrm{H}$ & 0.000827 \\
186 & $\mathrm{H}$ & -0.001421 \\
187 & $\mathrm{H}$ & -0.000005 \\
188 & $\mathrm{H}$ & 0.000002 \\
189 & $\mathrm{H}$ & 0.000026 \\
190 & $\mathrm{H}$ & -0.000009 \\
191 & $\mathrm{H}$ & 0.000005 \\
192 & $\mathrm{H}$ & -0.000006 \\
193 & $\mathrm{H}$ & 0.000001 \\
194 & $\mathrm{H}$ & 0.000002 \\
195 & $\mathrm{H}$ & 0.000004 \\
196 & $\mathrm{H}$ & -0.000067 \\
197 & $\mathrm{H}$ & 0.000447 \\
198 & $\mathrm{H}$ & -0.000027 \\
199 & $\mathrm{H}$ & -0.000019 \\
200 & $\mathrm{H}$ & -0.000080 \\
201 & $\mathrm{H}$ & 0.000480 \\
202 & $\mathrm{H}$ & 0.000045 \\
203 & $\mathrm{H}$ & 0.000026 \\
204 & $\mathrm{H}$ & -0.000041 \\
216 & $\mathrm{H}$ & 0.000028 \\
217 & $\mathrm{H}$ & -0.000027 \\
206 & $\mathrm{H}$ & 0.000447 \\
207 & $\mathrm{H}$ & -0.000067 \\
208 & $\mathrm{H}$ & 0.000045 \\
209 & $\mathrm{H}$ & -0.000041 \\
210 & $\mathrm{H}$ & 0.000026 \\
212 & $\mathrm{H}$ & -0.000019 \\
& $\mathrm{H}$ & -0.000080 \\
215 & 0.000479 \\
\hline & $\mathrm{H}$ & -0.000001 \\
\hline 16 &
\end{tabular}




$\begin{array}{lll}219 & \mathrm{H} & -0.000009 \\ 220 & \mathrm{H} & 0.000001 \\ 221 & \mathrm{H} & 0.000003 \\ 222 & \mathrm{H} & 0.000001\end{array}$

9a

Energy (with Zero Point Energy correction $)=-4563.050338(-4561.111220)$ Hartree Free energy $(298 \mathrm{~K})=-4561.259602$ Hartree

Zero number of imaginary frequency

01

$\begin{array}{lrrr}\text { C } & 11.48705300 & -2.35091300 & 1.28030400 \\ \mathrm{C} & 11.47870200 & -1.09398400 & 0.65842100 \\ \mathrm{C} & 10.23529500 & -0.48527100 & 0.32151600 \\ \mathrm{C} & 9.02528400 & -1.20018600 & 0.52236900 \\ \mathrm{C} & 9.09346200 & -2.42784600 & 1.19981200 \\ \mathrm{C} & 10.30427500 & -3.01655900 & 1.60024900 \\ \mathrm{C} & 10.21820600 & 0.83640800 & -0.22934000 \\ \mathrm{C} & 8.98991500 & 1.51952500 & -0.43272100 \\ \mathrm{C} & 7.72379300 & 0.85919700 & -0.04144700 \\ \mathrm{C} & 7.74393400 & -0.57639400 & 0.12183000 \\ \mathrm{C} & 11.44634200 & 1.47723100 & -0.56275500 \\ \mathrm{C} & 11.42384500 & 2.73469600 & -1.18289100 \\ \mathrm{C} & 10.22508300 & 3.37142500 & -1.50209700 \\ \mathrm{C} & 9.02872500 & 2.75130700 & -1.10575400 \\ \mathrm{C} & 12.70006000 & -0.40144700 & 0.35041000 \\ \mathrm{C} & 12.68467500 & 0.81621300 & -0.25294300 \\ \mathrm{C} & 6.50276300 & 1.54554000 & 0.11854800 \\ \mathrm{C} & 5.28003300 & 0.81256400 & 0.15046700 \\ \mathrm{C} & 5.30135200 & -0.60462000 & -0.09358900 \\ \mathrm{C} & 6.54662400 & -1.29972000 & -0.04966600 \\ \mathrm{C} & 4.01502900 & 1.44745500 & 0.36738500 \\ \mathrm{C} & 2.84303000 & 0.75732400 & 0.21438300 \\ \mathrm{C} & 2.85714700 & -0.64723100 & -0.18413400 \\ \mathrm{C} & 4.06331000 & -1.28681900 & -0.32453900 \\ \mathrm{C} & -0.70533900 & -4.63243500 & -1.34686000 \\ \mathrm{C} & 0.71177200 & -4.63334300 & -1.34574500 \\ \mathrm{C} & 1.40495700 & -3.49463900 & -1.01571400 \\ \mathrm{C} & 0.74239500 & -2.28239500 & -0.66552900 \\ \mathrm{C} & -0.73482800 & -2.28182000 & -0.66557000 \\ \mathrm{C} & -1.39805500 & -3.49330200 & -1.01719400 \\ \mathrm{C} & 1.45367800 & -1.09718100 & -0.32215300 \\ \mathrm{C} & 0.69282200 & 0.02595400 & 0.00575900 \\ \mathrm{C} & -2.84837500 & -0.64513200 & -0.18252000 \\ \mathrm{C} & & \end{array}$




\begin{tabular}{|c|c|c|c|}
\hline $\mathrm{C}$ & -2.83264000 & 0.76004700 & 0.21389500 \\
\hline $\mathrm{C}$ & -4.00374400 & 1.45194000 & 0.36514800 \\
\hline $\mathrm{C}$ & -5.26937600 & 0.81797900 & 0.14960000 \\
\hline $\mathrm{C}$ & -5.29257800 & -0.60014300 & -0.08902900 \\
\hline $\mathrm{C}$ & -4.05530400 & -1.28422800 & -0.31925900 \\
\hline $\mathrm{C}$ & -6.49092700 & 1.55252200 & 0.11466200 \\
\hline $\mathrm{C}$ & -7.71290700 & 0.86718700 & -0.04189700 \\
\hline $\mathrm{C}$ & -7.73534800 & -0.56757000 & 0.12825100 \\
\hline $\mathrm{C}$ & -6.53886200 & -1.29333200 & -0.04057400 \\
\hline $\mathrm{C}$ & -8.97786900 & 1.52817900 & -0.43510900 \\
\hline $\mathrm{C}$ & -10.20734700 & 0.84963600 & -0.22470600 \\
\hline $\mathrm{C}$ & -10.22628700 & -0.46901200 & 0.33333300 \\
\hline $\mathrm{C}$ & -9.01768500 & -1.18681600 & 0.53366300 \\
\hline $\mathrm{C}$ & -11.47062700 & -1.07164900 & 0.67786700 \\
\hline $\mathrm{C}$ & -11.48109700 & -2.32508000 & 1.30645300 \\
\hline $\mathrm{C}$ & -10.29954900 & -2.99424900 & 1.62356100 \\
\hline $\mathrm{C}$ & -9.08808800 & -2.41205600 & 1.21577800 \\
\hline $\mathrm{C}$ & -9.01469100 & 2.75594600 & -1.11553000 \\
\hline $\mathrm{C}$ & -10.21025300 & 3.37707300 & -1.51257300 \\
\hline $\mathrm{C}$ & -11.41000800 & 2.74585200 & -1.18607900 \\
\hline $\mathrm{C}$ & -11.43452300 & 1.49224400 & -0.55827700 \\
\hline $\mathrm{C}$ & -12.67388100 & 0.83702700 & -0.24042400 \\
\hline $\mathrm{C}$ & -12.69088200 & -0.37689700 & 0.37030200 \\
\hline $\mathrm{C}$ & 6.54058200 & -2.77712200 & -0.29135200 \\
\hline $\mathrm{C}$ & 10.36077600 & -4.36589800 & 2.34351600 \\
\hline $\mathrm{C}$ & 10.99461100 & -5.42498400 & 1.40994900 \\
\hline $\mathrm{C}$ & 8.96716000 & -4.86621700 & 2.76808100 \\
\hline $\mathrm{C}$ & 11.22197300 & -4.22899400 & 3.62141900 \\
\hline $\mathrm{C}$ & 10.25100600 & 4.72051300 & -2.24761800 \\
\hline $\mathrm{C}$ & 8.84334100 & 5.21011100 & -2.63605100 \\
\hline $\mathrm{C}$ & 11.07997200 & 4.58467600 & -3.54694100 \\
\hline $\mathrm{C}$ & 10.90088700 & 5.78786500 & -1.33486400 \\
\hline $\mathrm{C}$ & 6.44878800 & 3.02202800 & 0.35688800 \\
\hline $\mathrm{C}$ & -6.53606600 & -2.77203500 & -0.27302900 \\
\hline $\mathrm{C}$ & -6.43483500 & 3.03004500 & 0.34599300 \\
\hline $\mathrm{C}$ & -10.36013700 & -4.33664900 & 2.37899700 \\
\hline $\mathrm{C}$ & -11.04656700 & -5.39114700 & 1.47813700 \\
\hline $\mathrm{C}$ & -11.17658300 & -4.16739700 & 3.68234100 \\
\hline $\mathrm{C}$ & -8.96488900 & -4.86436600 & 2.76238700 \\
\hline $\mathrm{C}$ & -10.23426500 & 4.72157000 & -2.26637600 \\
\hline $\mathrm{C}$ & -8.82619300 & 5.20483000 & -2.66124500 \\
\hline $\mathrm{C}$ & -10.87900700 & 5.79635500 & -1.35871500 \\
\hline $\mathrm{C}$ & -11.06677200 & 4.57995200 & -3.56282300 \\
\hline $\mathrm{C}$ & 5.83818700 & -3.67671900 & 0.52864000 \\
\hline
\end{tabular}




\begin{tabular}{|c|c|c|c|}
\hline $\mathrm{C}$ & 5.80472100 & -5.03880300 & 0.23973000 \\
\hline $\mathrm{C}$ & 6.46520400 & -5.57506800 & -0.88002700 \\
\hline $\mathrm{C}$ & 7.16569300 & -4.67239900 & -1.69198000 \\
\hline $\mathrm{C}$ & 7.20160300 & -3.30639100 & -1.40685300 \\
\hline $\mathrm{C}$ & 5.72792600 & 3.89658300 & -0.46744100 \\
\hline $\mathrm{C}$ & 5.65740000 & 5.26404700 & -0.18952600 \\
\hline $\mathrm{C}$ & 6.29905400 & 5.82054100 & 0.92554400 \\
\hline $\mathrm{C}$ & 7.01785300 & 4.93631500 & 1.74951900 \\
\hline $\mathrm{C}$ & 7.09213600 & 3.57429100 & 1.47631300 \\
\hline $\mathrm{C}$ & -7.07768400 & 3.58884900 & 1.46244900 \\
\hline $\mathrm{C}$ & -7.00139700 & 4.95210300 & 1.72890100 \\
\hline $\mathrm{C}$ & -6.28102900 & 5.83113100 & 0.90074200 \\
\hline $\mathrm{C}$ & -5.63985800 & 5.26811200 & -0.21132800 \\
\hline $\mathrm{C}$ & -5.71234200 & 3.89938700 & -0.48241600 \\
\hline $\mathrm{C}$ & -7.20598200 & -3.30680200 & -1.38531400 \\
\hline $\mathrm{C}$ & -7.17659100 & -4.67124600 & -1.65556700 \\
\hline $\mathrm{C}$ & -6.47835900 & -5.57549700 & -0.83579100 \\
\hline $\mathrm{C}$ & -5.81023600 & -5.03671200 & 0.27261800 \\
\hline $\mathrm{C}$ & -5.83619600 & -3.66702400 & 0.54775900 \\
\hline $\mathrm{C}$ & 6.38738500 & -7.08402100 & -1.17329700 \\
\hline $\mathrm{C}$ & 7.18569300 & -7.47804300 & -2.43041400 \\
\hline $\mathrm{C}$ & 4.90939600 & -7.48667400 & -1.39471400 \\
\hline $\mathrm{C}$ & 6.95766400 & -7.87672600 & 0.02675400 \\
\hline $\mathrm{C}$ & 6.23487800 & 7.31888500 & 1.27133400 \\
\hline $\mathrm{C}$ & 5.42163200 & 8.12383000 & 0.24000600 \\
\hline $\mathrm{C}$ & 7.66726600 & 7.90228600 & 1.31595200 \\
\hline $\mathrm{C}$ & 5.56815400 & 7.49660600 & 2.65659100 \\
\hline $\mathrm{C}$ & -6.46255500 & -7.07572800 & -1.17895700 \\
\hline $\mathrm{C}$ & -5.66806800 & -7.90349200 & -0.15107900 \\
\hline $\mathrm{C}$ & -5.81028500 & -7.27604900 & -2.56800800 \\
\hline $\mathrm{C}$ & -7.91240300 & -7.61494900 & -1.21362400 \\
\hline $\mathrm{C}$ & -6.21459800 & 7.33107300 & 1.23909500 \\
\hline $\mathrm{C}$ & -7.64609100 & 7.91685700 & 1.28098700 \\
\hline $\mathrm{C}$ & -5.40026300 & 8.12968700 & 0.20370800 \\
\hline $\mathrm{C}$ & -5.54744100 & 7.51462700 & 2.62338500 \\
\hline $\mathrm{C}$ & 1.43887400 & 1.18301700 & 0.34550800 \\
\hline $\mathrm{C}$ & 0.72547400 & 2.32465600 & 0.67991600 \\
\hline $\mathrm{C}$ & -0.71378200 & 2.32536300 & 0.67954300 \\
\hline $\mathrm{C}$ & -1.42817300 & 1.18435500 & 0.34506300 \\
\hline $\mathrm{H}$ & 12.45012900 & -2.78969200 & 1.52831700 \\
\hline $\mathrm{H}$ & 8.17183400 & -2.93304500 & 1.43936400 \\
\hline $\mathrm{H}$ & 12.37580800 & 3.19767900 & -1.43008500 \\
\hline $\mathrm{H}$ & 8.09528300 & 3.23409100 & -1.34550200 \\
\hline $\mathrm{H}$ & 13.64227300 & -0.88377200 & 0.59995700 \\
\hline
\end{tabular}




\begin{tabular}{|c|c|c|c|}
\hline $\mathrm{H}$ & 13.61444100 & 1.32299800 & -0.50069300 \\
\hline $\mathrm{H}$ & 3.99109600 & 2.49500900 & 0.64192200 \\
\hline $\mathrm{H}$ & 4.10277400 & -2.33044600 & -0.60179500 \\
\hline $\mathrm{H}$ & -1.24541600 & -5.53785100 & -1.61049600 \\
\hline $\mathrm{H}$ & 1.25165100 & -5.53936300 & -1.60768900 \\
\hline $\mathrm{H}$ & -2.48195600 & -3.51062000 & -1.02240300 \\
\hline $\mathrm{H}$ & -3.97868500 & 2.50008300 & 0.63734600 \\
\hline $\mathrm{H}$ & -4.09620800 & -2.32897200 & -0.59217700 \\
\hline $\mathrm{H}$ & -12.44468300 & -2.75852200 & 1.56208300 \\
\hline $\mathrm{H}$ & -8.16761500 & -2.92008700 & 1.45355600 \\
\hline $\mathrm{H}$ & -8.08038200 & 3.23448900 & -1.36044200 \\
\hline $\mathrm{H}$ & -12.36127300 & 3.21023600 & -1.43332700 \\
\hline $\mathrm{H}$ & -13.60291400 & 1.34520400 & -0.48806700 \\
\hline $\mathrm{H}$ & -13.63367900 & -0.85484900 & 0.62597700 \\
\hline $\mathrm{H}$ & 10.39423900 & -5.55149200 & 0.50167300 \\
\hline $\mathrm{H}$ & 12.00727700 & -5.13992900 & 1.10426500 \\
\hline $\mathrm{H}$ & 11.05740900 & -6.39608100 & 1.91708700 \\
\hline $\mathrm{H}$ & 8.31621500 & -5.05616800 & 1.90887300 \\
\hline $\mathrm{H}$ & 8.46310400 & -4.14889400 & 3.42629300 \\
\hline $\mathrm{H}$ & 9.06857200 & -5.80781300 & 3.32015000 \\
\hline $\mathrm{H}$ & 12.25207500 & -3.93363800 & 3.39775100 \\
\hline $\mathrm{H}$ & 10.79929600 & -3.47960000 & 4.30037700 \\
\hline $\mathrm{H}$ & 11.26183900 & -5.18649300 & 4.15501000 \\
\hline $\mathrm{H}$ & 8.21165900 & 5.38841700 & -1.76000000 \\
\hline $\mathrm{H}$ & 8.92326200 & 6.15548300 & -3.18533100 \\
\hline $\mathrm{H}$ & 8.32959000 & 4.49249600 & -3.28630800 \\
\hline $\mathrm{H}$ & 10.64323800 & 3.83199300 & -4.21325800 \\
\hline $\mathrm{H}$ & 11.10294400 & 5.54105600 & -4.08365000 \\
\hline $\mathrm{H}$ & 12.11612800 & 4.29283800 & -3.34761400 \\
\hline $\mathrm{H}$ & 11.92340100 & 5.51292100 & -1.05407100 \\
\hline $\mathrm{H}$ & 10.94333800 & 6.75688500 & -1.84800100 \\
\hline $\mathrm{H}$ & 10.32321500 & 5.91500500 & -0.41216800 \\
\hline $\mathrm{H}$ & -10.47855100 & -5.54194500 & 0.55300200 \\
\hline $\mathrm{H}$ & -11.11416000 & -6.35470900 & 1.99879300 \\
\hline $\mathrm{H}$ & -12.06230400 & -5.08847100 & 1.20133500 \\
\hline $\mathrm{H}$ & -10.71396800 & -3.42294500 & 4.34038500 \\
\hline $\mathrm{H}$ & -12.20506200 & -3.84699000 & 3.48714500 \\
\hline $\mathrm{H}$ & -11.22392600 & -5.11881000 & 4.22623000 \\
\hline $\mathrm{H}$ & -8.42637200 & -4.15755100 & 3.40438200 \\
\hline $\mathrm{H}$ & -9.06882700 & -5.80315600 & 3.31886900 \\
\hline $\mathrm{H}$ & -8.34419400 & -5.06712700 & 1.88377900 \\
\hline $\mathrm{H}$ & -8.31604100 & 4.48177800 & -3.30830700 \\
\hline $\mathrm{H}$ & -8.90483600 & 6.14700300 & -3.21617200 \\
\hline $\mathrm{H}$ & -8.19187800 & 5.38679100 & -1.78784700 \\
\hline
\end{tabular}




\begin{tabular}{|c|c|c|c|}
\hline $\mathrm{H}$ & -11.90165500 & 5.52606900 & -1.07391100 \\
\hline $\mathrm{H}$ & -10.29886100 & 5.92749400 & -0.43813600 \\
\hline $\mathrm{H}$ & -10.91988900 & 6.76232500 & -1.87769700 \\
\hline $\mathrm{H}$ & -10.63366300 & 3.82202400 & -4.22555200 \\
\hline $\mathrm{H}$ & -11.08852400 & 5.53306800 & -4.10535600 \\
\hline $\mathrm{H}$ & -12.10320300 & 4.29209100 & -3.35917200 \\
\hline $\mathrm{H}$ & 5.31682900 & -3.30391700 & 1.40638100 \\
\hline $\mathrm{H}$ & 5.24918200 & -5.69375100 & 0.90563100 \\
\hline $\mathrm{H}$ & 7.69129800 & -5.02482000 & -2.57266100 \\
\hline $\mathrm{H}$ & 7.74946900 & -2.63769200 & -2.06470400 \\
\hline $\mathrm{H}$ & 5.22066600 & 3.50628900 & -1.34587600 \\
\hline $\mathrm{H}$ & 5.08902200 & 5.89465300 & -0.86447300 \\
\hline $\mathrm{H}$ & 7.52829400 & 5.31404700 & 2.63136200 \\
\hline $\mathrm{H}$ & 7.65422700 & 2.92275300 & 2.13931700 \\
\hline $\mathrm{H}$ & -7.64099600 & 2.94148900 & 2.12850100 \\
\hline $\mathrm{H}$ & -7.51149700 & 5.33498500 & 2.60871800 \\
\hline $\mathrm{H}$ & -5.07027700 & 5.89446900 & -0.88921100 \\
\hline $\mathrm{H}$ & -5.20529700 & 3.50392900 & -1.35866200 \\
\hline $\mathrm{H}$ & -7.75271100 & -2.63965400 & -2.04562700 \\
\hline $\mathrm{H}$ & -7.70601100 & -5.03479400 & -2.53215900 \\
\hline $\mathrm{H}$ & -5.25621100 & -5.68294600 & 0.94468100 \\
\hline $\mathrm{H}$ & -5.30898000 & -3.29079800 & 1.42054300 \\
\hline $\mathrm{H}$ & 6.80654000 & -6.97796800 & -3.32881300 \\
\hline $\mathrm{H}$ & 7.10437200 & -8.55832100 & -2.59538200 \\
\hline $\mathrm{H}$ & 8.25032400 & -7.23862800 & -2.32887500 \\
\hline $\mathrm{H}$ & 4.48066900 & -6.94751600 & -2.24742600 \\
\hline $\mathrm{H}$ & 4.83284800 & -8.56166400 & -1.59914400 \\
\hline $\mathrm{H}$ & 4.29254400 & -7.26983000 & -0.51612500 \\
\hline $\mathrm{H}$ & 8.00830400 & -7.61920300 & 0.20299600 \\
\hline $\mathrm{H}$ & 6.40478800 & -7.67486800 & 0.95010600 \\
\hline $\mathrm{H}$ & 6.89998400 & -8.95488100 & -0.16678000 \\
\hline $\mathrm{H}$ & 5.85649500 & 8.05783600 & -0.76383800 \\
\hline $\mathrm{H}$ & 4.38095400 & 7.78449900 & 0.18531300 \\
\hline $\mathrm{H}$ & 5.40832900 & 9.18178400 & 0.52525600 \\
\hline $\mathrm{H}$ & 8.16390100 & 7.79835600 & 0.34438200 \\
\hline $\mathrm{H}$ & 8.28939200 & 7.40072500 & 2.06449900 \\
\hline $\mathrm{H}$ & 7.63662800 & 8.96905300 & 1.56955300 \\
\hline $\mathrm{H}$ & 4.54630200 & 7.10028600 & 2.65365700 \\
\hline $\mathrm{H}$ & 6.12427600 & 6.97948000 & 3.44548000 \\
\hline $\mathrm{H}$ & 5.51995600 & 8.55948600 & 2.92355200 \\
\hline $\mathrm{H}$ & -4.61698200 & -7.59668400 & -0.10352300 \\
\hline $\mathrm{H}$ & -5.68957100 & -8.96193300 & -0.43399600 \\
\hline $\mathrm{H}$ & -6.09411000 & -7.82185100 & 0.85537000 \\
\hline $\mathrm{H}$ & -4.77682000 & -6.91084600 & -2.57222600 \\
\hline
\end{tabular}




$\begin{array}{lrrr}\mathrm{H} & -6.35513700 & -6.74343100 & -3.35442800 \\ \mathrm{H} & -5.79590000 & -8.34031600 & -2.83335000 \\ \mathrm{H} & -8.52347900 & -7.09613200 & -1.95948600 \\ \mathrm{H} & -7.91611900 & -8.68267800 & -1.46493900 \\ \mathrm{H} & -8.39952200 & -7.49380100 & -0.23925400 \\ \mathrm{H} & -8.26888100 & 7.41998600 & 2.03210400 \\ \mathrm{H} & -7.61380500 & 8.98482700 & 1.52925900 \\ \mathrm{H} & -8.14301100 & 7.80883800 & 0.31000900 \\ \mathrm{H} & -5.83529900 & 8.05930800 & -0.79976300 \\ \mathrm{H} & -5.38540700 & 9.18903000 & 0.48367900 \\ \mathrm{H} & -4.36008000 & 7.78858800 & 0.15063200 \\ \mathrm{H} & -4.52620300 & 7.11671800 & 2.62229500 \\ \mathrm{H} & -5.49756700 & 8.57874000 & 2.88507800 \\ \mathrm{H} & -6.10427500 & 7.00226300 & 3.41487600 \\ \mathrm{H} & 1.23542800 & 3.24553500 & 0.95181900 \\ \mathrm{H} & -1.22296500 & 3.24677100 & 0.95109600 \\ \mathrm{H} & 2.48881600 & -3.51332200 & -1.01888100 \\ & & & \\ & & 9 \mathbf{a}^{\cdot+} & \end{array}$

Energy (with Zero Point Energy correction $)=-4562.851774(-4560.912560)$ Hartree Free energy $(298 \mathrm{~K})=-4561.061833$ Hartree

Zero number of imaginary frequency

12

$\begin{array}{lrrr}\text { C } & 11.47407500 & -2.36055200 & 1.27106200 \\ \mathrm{C} & 11.46875100 & -1.11073400 & 0.63815200 \\ \mathrm{C} & 10.22365500 & -0.49917000 & 0.30837200 \\ \mathrm{C} & 9.01217700 & -1.20876700 & 0.53144600 \\ \mathrm{C} & 9.07900200 & -2.43417100 & 1.21398300 \\ \mathrm{C} & 10.29092800 & -3.02182600 & 1.60820000 \\ \mathrm{C} & 10.20604900 & 0.81199600 & -0.25654700 \\ \mathrm{C} & 8.97697700 & 1.50220400 & -0.45716200 \\ \mathrm{C} & 7.72269100 & 0.84984200 & -0.05418600 \\ \mathrm{C} & 7.73827100 & -0.57748100 & 0.14116700 \\ \mathrm{C} & 11.43359300 & 1.44707600 & -0.60390500 \\ \mathrm{C} & 11.40589100 & 2.69787700 & -1.23398700 \\ \mathrm{C} & 10.20573500 & 3.34200200 & -1.54971900 \\ \mathrm{C} & 9.01258300 & 2.73451600 & -1.13671200 \\ \mathrm{C} & 12.69035700 & -0.42632500 & 0.31311000 \\ \mathrm{C} & 12.67372400 & 0.78619000 & -0.29984600 \\ \mathrm{C} & 6.49562700 & 1.55137300 & 0.11963300 \\ \mathrm{C} & 5.27745600 & 0.82643300 & 0.15654600 \\ \mathrm{C} & 5.29285100 & -0.59201000 & -0.08450900 \\ \mathrm{C} & 6.53135800 & -1.29762300 & -0.03577900 \\ \mathrm{C} & 4.01385200 & 1.46616000 & 0.38546900\end{array}$




\begin{tabular}{|c|c|c|c|}
\hline $\mathrm{C}$ & 2.83804100 & 0.78343400 & 0.22606100 \\
\hline $\mathrm{C}$ & 2.84694500 & -0.61691000 & -0.18636400 \\
\hline $\mathrm{C}$ & 4.05797500 & -1.26420600 & -0.32657800 \\
\hline $\mathrm{C}$ & -0.70092200 & -4.59280100 & -1.39257700 \\
\hline $\mathrm{C}$ & 0.70897700 & -4.59393100 & -1.39068500 \\
\hline $\mathrm{C}$ & 1.40350100 & -3.45317200 & -1.04645000 \\
\hline $\mathrm{C}$ & 0.74050600 & -2.25257500 & -0.68572600 \\
\hline $\mathrm{C}$ & -0.73148200 & -2.25186000 & -0.68628200 \\
\hline $\mathrm{C}$ & -1.39505800 & -3.45146800 & -1.04927600 \\
\hline $\mathrm{C}$ & 1.45761700 & -1.06226600 & -0.32885300 \\
\hline $\mathrm{C}$ & 0.69240400 & 0.06201800 & 0.01022300 \\
\hline $\mathrm{C}$ & -0.68133700 & 0.06259100 & 0.01002100 \\
\hline $\mathrm{C}$ & -1.44772400 & -1.06109900 & -0.32880400 \\
\hline $\mathrm{C}$ & -2.83672100 & -0.61452400 & -0.18551300 \\
\hline $\mathrm{C}$ & -2.82601300 & 0.78658400 & 0.22453200 \\
\hline $\mathrm{C}$ & -4.00078300 & 1.47154900 & 0.38143900 \\
\hline $\mathrm{C}$ & -5.26506100 & 0.83293600 & 0.15397500 \\
\hline $\mathrm{C}$ & -5.28264500 & -0.58668100 & -0.08034100 \\
\hline $\mathrm{C}$ & -4.04875900 & -1.26121900 & -0.32162700 \\
\hline $\mathrm{C}$ & -6.48179800 & 1.55981300 & 0.11293500 \\
\hline $\mathrm{C}$ & -7.71006400 & 0.85941400 & -0.05630300 \\
\hline $\mathrm{C}$ & -7.72814900 & -0.56652400 & 0.14809100 \\
\hline $\mathrm{C}$ & -6.52236900 & -1.28991600 & -0.02593500 \\
\hline $\mathrm{C}$ & -8.96319400 & 1.51195900 & -0.46200900 \\
\hline $\mathrm{C}$ & -10.19354400 & 0.82670100 & -0.25334700 \\
\hline $\mathrm{C}$ & -10.21305800 & -0.48023300 & 0.32121300 \\
\hline $\mathrm{C}$ & -9.00292700 & -1.19217400 & 0.54522300 \\
\hline $\mathrm{C}$ & -11.45907400 & -1.08505800 & 0.65989800 \\
\hline $\mathrm{C}$ & -11.46639600 & -2.32981400 & 1.30249700 \\
\hline $\mathrm{C}$ & -10.28434400 & -2.99338700 & 1.63897700 \\
\hline $\mathrm{C}$ & -9.07178800 & -2.41321900 & 1.23576300 \\
\hline $\mathrm{C}$ & -8.99671600 & 2.73922600 & -1.15066400 \\
\hline $\mathrm{C}$ & -10.18911000 & 3.34696900 & -1.56542800 \\
\hline $\mathrm{C}$ & -11.39034800 & 2.70877500 & -1.24171600 \\
\hline $\mathrm{C}$ & -11.42013700 & 1.46290700 & -0.60203200 \\
\hline $\mathrm{C}$ & -12.66133200 & 0.80828000 & -0.28896500 \\
\hline $\mathrm{C}$ & -12.67962300 & -0.39924100 & 0.33369600 \\
\hline $\mathrm{C}$ & 6.52197200 & -2.77079900 & -0.28219600 \\
\hline $\mathrm{C}$ & 10.35325900 & -4.36094300 & 2.36764000 \\
\hline $\mathrm{C}$ & 11.01184500 & -5.42352200 & 1.45502300 \\
\hline $\mathrm{C}$ & 8.95985400 & -4.87187500 & 2.77948600 \\
\hline $\mathrm{C}$ & 11.19699700 & -4.19466400 & 3.65403000 \\
\hline $\mathrm{C}$ & 10.23655900 & 4.68033700 & -2.31216500 \\
\hline $\mathrm{C}$ & 8.82779200 & 5.18725100 & -2.67264400 \\
\hline
\end{tabular}




\begin{tabular}{|c|c|c|c|}
\hline $\mathrm{C}$ & 11.03287600 & 4.51080000 & -3.62817800 \\
\hline $\mathrm{C}$ & 10.92600500 & 5.74741800 & -1.42815100 \\
\hline $\mathrm{C}$ & 6.46480600 & 3.02252200 & 0.37230100 \\
\hline $\mathrm{C}$ & -6.51761200 & -2.76456600 & -0.26151400 \\
\hline $\mathrm{C}$ & -6.44834800 & 3.03244800 & 0.35623600 \\
\hline $\mathrm{C}$ & -10.34999200 & -4.32392800 & 2.41310600 \\
\hline $\mathrm{C}$ & -11.04937500 & -5.38584200 & 1.53068600 \\
\hline $\mathrm{C}$ & -11.15849700 & -4.12722400 & 3.71786600 \\
\hline $\mathrm{C}$ & -8.95585000 & -4.85507000 & 2.79547500 \\
\hline $\mathrm{C}$ & -10.21792900 & 4.67954200 & -2.33795000 \\
\hline $\mathrm{C}$ & -8.80855400 & 5.17975100 & -2.70539900 \\
\hline $\mathrm{C}$ & -10.90244500 & 5.75523100 & -1.46056300 \\
\hline $\mathrm{C}$ & -11.01767200 & 4.50222600 & -3.65086500 \\
\hline $\mathrm{C}$ & 5.79624500 & -3.66664000 & 0.52266300 \\
\hline $\mathrm{C}$ & 5.76510300 & -5.02815000 & 0.23147000 \\
\hline $\mathrm{C}$ & 6.44493100 & -5.56494700 & -0.87657600 \\
\hline $\mathrm{C}$ & 7.16644900 & -4.66371100 & -1.67319300 \\
\hline $\mathrm{C}$ & 7.20780400 & -3.29974400 & -1.38386100 \\
\hline $\mathrm{C}$ & 5.72135900 & 3.90945900 & -0.41944800 \\
\hline $\mathrm{C}$ & 5.67733800 & 5.27502700 & -0.12860600 \\
\hline $\mathrm{C}$ & 6.36349300 & 5.81510900 & 0.96816300 \\
\hline $\mathrm{C}$ & 7.10374800 & 4.91658600 & 1.75866500 \\
\hline $\mathrm{C}$ & 7.15926500 & 3.55781900 & 1.47060100 \\
\hline $\mathrm{C}$ & -7.14247800 & 3.57642700 & 1.45047600 \\
\hline $\mathrm{C}$ & -7.08463600 & 4.93700400 & 1.72936500 \\
\hline $\mathrm{C}$ & -6.34233200 & 5.82882300 & 0.93319800 \\
\hline $\mathrm{C}$ & -5.65644600 & 5.28006800 & -0.15943000 \\
\hline $\mathrm{C}$ & -5.70272400 & 3.91263800 & -0.44099000 \\
\hline $\mathrm{C}$ & -7.21661400 & -3.30018200 & -1.35651700 \\
\hline $\mathrm{C}$ & -7.18558600 & -4.66335200 & -1.62761400 \\
\hline $\mathrm{C}$ & -6.46591200 & -5.56600000 & -0.82292000 \\
\hline $\mathrm{C}$ & -5.77362400 & -5.02541100 & 0.26980000 \\
\hline $\mathrm{C}$ & -5.79350700 & -3.65547900 & 0.54385400 \\
\hline $\mathrm{C}$ & 6.37026200 & -7.07310200 & -1.17269400 \\
\hline $\mathrm{C}$ & 7.18716400 & -7.46621700 & -2.41803400 \\
\hline $\mathrm{C}$ & 4.89459800 & -7.47234500 & -1.41636000 \\
\hline $\mathrm{C}$ & 6.92096600 & -7.86578800 & 0.03679400 \\
\hline $\mathrm{C}$ & 6.32873600 & 7.31067200 & 1.32712300 \\
\hline $\mathrm{C}$ & 5.48579700 & 8.13182700 & 0.33321500 \\
\hline $\mathrm{C}$ & 7.76918000 & 7.87633800 & 1.32172600 \\
\hline $\mathrm{C}$ & 5.71717400 & 7.48199300 & 2.73870900 \\
\hline $\mathrm{C}$ & -6.45904900 & -7.06651800 & -1.16258900 \\
\hline $\mathrm{C}$ & -5.63773700 & -7.89138600 & -0.15376700 \\
\hline $\mathrm{C}$ & -5.84443100 & -7.26845000 & -2.56873100 \\
\hline
\end{tabular}




\begin{tabular}{|c|c|c|c|}
\hline $\mathrm{C}$ & -7.91041900 & -7.60356300 & -1.15743200 \\
\hline $\mathrm{C}$ & -6.30519700 & 7.32674600 & 1.28191600 \\
\hline $\mathrm{C}$ & -7.74467400 & 7.89480700 & 1.27186500 \\
\hline $\mathrm{C}$ & -5.46034200 & 8.13962100 & 0.28284000 \\
\hline $\mathrm{C}$ & -5.69408500 & 7.50672300 & 2.69261800 \\
\hline $\mathrm{C}$ & 1.43883400 & 1.20898700 & 0.36021500 \\
\hline $\mathrm{C}$ & 0.72110800 & 2.35501200 & 0.70681500 \\
\hline $\mathrm{C}$ & -0.70774900 & 2.35582900 & 0.70611500 \\
\hline $\mathrm{C}$ & -1.42652500 & 1.21055800 & 0.35914600 \\
\hline $\mathrm{H}$ & 12.43608300 & -2.80186300 & 1.51671800 \\
\hline $\mathrm{H}$ & 8.15804600 & -2.93533200 & 1.46539700 \\
\hline $\mathrm{H}$ & 12.35589800 & 3.15585000 & -1.49565800 \\
\hline $\mathrm{H}$ & 8.07926300 & 3.22042800 & -1.37072000 \\
\hline $\mathrm{H}$ & 13.63261700 & -0.90946200 & 0.55836800 \\
\hline $\mathrm{H}$ & 13.60217100 & 1.28793000 & -0.55990100 \\
\hline $\mathrm{H}$ & 3.99941300 & 2.50956600 & 0.67593700 \\
\hline $\mathrm{H}$ & 4.09554600 & -2.30552700 & -0.61283400 \\
\hline $\mathrm{H}$ & -1.24284600 & -5.49325100 & -1.66631400 \\
\hline $\mathrm{H}$ & 1.25084300 & -5.49517600 & -1.66198500 \\
\hline $\mathrm{H}$ & -2.47844900 & -3.47108200 & -1.05689000 \\
\hline $\mathrm{H}$ & -3.98499200 & 2.51577700 & 0.66890100 \\
\hline $\mathrm{H}$ & -4.08809700 & -2.30382900 & -0.60316000 \\
\hline $\mathrm{H}$ & -12.42895300 & -2.76532300 & 1.55639700 \\
\hline $\mathrm{H}$ & -8.15179700 & -2.91627000 & 1.48680500 \\
\hline $\mathrm{H}$ & -8.06246700 & 3.22061400 & -1.39037200 \\
\hline $\mathrm{H}$ & -12.33966600 & 3.16764600 & -1.50431000 \\
\hline $\mathrm{H}$ & -13.58908000 & 1.31080700 & -0.54999000 \\
\hline $\mathrm{H}$ & -13.62253000 & -0.87751400 & 0.58589900 \\
\hline $\mathrm{H}$ & 10.42576000 & -5.57047800 & 0.54043000 \\
\hline $\mathrm{H}$ & 12.02591100 & -5.13370700 & 1.15960000 \\
\hline $\mathrm{H}$ & 11.07822500 & -6.38572700 & 1.97700200 \\
\hline $\mathrm{H}$ & 8.32141600 & -5.07551300 & 1.91349600 \\
\hline $\mathrm{H}$ & 8.44072400 & -4.15695500 & 3.42868600 \\
\hline $\mathrm{H}$ & 9.06492100 & -5.80823400 & 3.33859300 \\
\hline $\mathrm{H}$ & 12.22578400 & -3.88897900 & 3.43880000 \\
\hline $\mathrm{H}$ & 10.75632500 & -3.44327700 & 4.31906100 \\
\hline $\mathrm{H}$ & 11.24327700 & -5.14461200 & 4.19929800 \\
\hline $\mathrm{H}$ & 8.21973800 & 5.38427900 & -1.78352800 \\
\hline $\mathrm{H}$ & 8.90978700 & 6.12600200 & -3.23165200 \\
\hline $\mathrm{H}$ & 8.28836300 & 4.47332700 & -3.30617800 \\
\hline $\mathrm{H}$ & 10.56826900 & 3.75781200 & -4.27488100 \\
\hline $\mathrm{H}$ & 11.05962900 & 5.45933500 & -4.17719400 \\
\hline $\mathrm{H}$ & 12.06863400 & 4.20500900 & -3.44904000 \\
\hline $\mathrm{H}$ & 11.95091400 & 5.46179300 & -1.16827500 \\
\hline
\end{tabular}




\begin{tabular}{|c|c|c|c|}
\hline $\mathrm{H}$ & 10.97194400 & 6.70703600 & -1.95693300 \\
\hline $\mathrm{H}$ & 10.37305900 & 5.89863200 & -0.49398400 \\
\hline $\mathrm{H}$ & -10.48889000 & -5.55574500 & 0.60421100 \\
\hline $\mathrm{H}$ & -11.11953100 & -6.33974200 & 2.06718600 \\
\hline $\mathrm{H}$ & -12.06549900 & -5.08295300 & 1.25648400 \\
\hline $\mathrm{H}$ & -10.68724400 & -3.37724000 & 4.36323900 \\
\hline $\mathrm{H}$ & -12.18577900 & -3.80307800 & 3.52314400 \\
\hline $\mathrm{H}$ & -11.21015000 & -5.06993400 & 4.27516200 \\
\hline $\mathrm{H}$ & -8.41009000 & -4.14612900 & 3.42914500 \\
\hline $\mathrm{H}$ & -9.06309900 & -5.78689700 & 3.36178600 \\
\hline $\mathrm{H}$ & -8.34105700 & -5.07213100 & 1.91561900 \\
\hline $\mathrm{H}$ & -8.27256300 & 4.45954500 & -3.33472700 \\
\hline $\mathrm{H}$ & -8.88918300 & 6.11447500 & -3.27130700 \\
\hline $\mathrm{H}$ & -8.19794000 & 5.38179000 & -1.81915900 \\
\hline $\mathrm{H}$ & -11.92762100 & 5.47453500 & -1.19640600 \\
\hline $\mathrm{H}$ & -10.34710400 & 5.91187200 & -0.52871600 \\
\hline $\mathrm{H}$ & -10.94675400 & 6.71096800 & -1.99646300 \\
\hline $\mathrm{H}$ & -10.55653300 & 3.74314800 & -4.29291600 \\
\hline $\mathrm{H}$ & -11.04314300 & 5.44666500 & -4.20695600 \\
\hline $\mathrm{H}$ & -12.05383000 & 4.20055500 & -3.46708700 \\
\hline $\mathrm{H}$ & 5.26712600 & -3.29485500 & 1.39636700 \\
\hline $\mathrm{H}$ & 5.19951100 & -5.68309300 & 0.88825400 \\
\hline $\mathrm{H}$ & 7.70721200 & -5.01803400 & -2.54341400 \\
\hline $\mathrm{H}$ & 7.77517700 & -2.63449500 & -2.02829500 \\
\hline $\mathrm{H}$ & 5.18666100 & 3.53385500 & -1.28808200 \\
\hline $\mathrm{H}$ & 5.09645700 & 5.91865200 & -0.77972200 \\
\hline $\mathrm{H}$ & 7.64865400 & 5.28227900 & 2.62415500 \\
\hline $\mathrm{H}$ & 7.74091400 & 2.89746800 & 2.10729500 \\
\hline $\mathrm{H}$ & -7.72572100 & 2.92148200 & 2.09127800 \\
\hline $\mathrm{H}$ & -7.62935200 & 5.30952900 & 2.59205400 \\
\hline $\mathrm{H}$ & -5.07396500 & 5.91819300 & -0.81451600 \\
\hline $\mathrm{H}$ & -5.16806800 & 3.53015000 & -1.30663700 \\
\hline $\mathrm{H}$ & -7.78350500 & -2.63679400 & -2.00321600 \\
\hline $\mathrm{H}$ & -7.73401100 & -5.02925400 & -2.49080900 \\
\hline $\mathrm{H}$ & -5.20856100 & -5.67161100 & 0.93224900 \\
\hline $\mathrm{H}$ & -5.25540200 & -3.27933500 & 1.41018700 \\
\hline $\mathrm{H}$ & 6.82343200 & -6.96505900 & -3.32224600 \\
\hline $\mathrm{H}$ & 7.10575100 & -8.54557400 & -2.58547600 \\
\hline $\mathrm{H}$ & 8.25094900 & -7.23146300 & -2.29950400 \\
\hline $\mathrm{H}$ & 4.47947000 & -6.93308900 & -2.27597200 \\
\hline $\mathrm{H}$ & 4.82133900 & -8.54643200 & -1.62337300 \\
\hline $\mathrm{H}$ & 4.26489300 & -7.25839400 & -0.54588100 \\
\hline $\mathrm{H}$ & 7.96880300 & -7.60971900 & 0.23001100 \\
\hline $\mathrm{H}$ & 6.35296100 & -7.66667900 & 0.95160800 \\
\hline
\end{tabular}




$\begin{array}{lrrr}\mathrm{H} & 6.86651300 & -8.94289700 & -0.16003700 \\ \mathrm{H} & 5.88209800 & 8.07172600 & -0.68683200 \\ \mathrm{H} & 4.43963200 & 7.80514700 & 0.31550400 \\ \mathrm{H} & 5.49475700 & 9.18677800 & 0.62757400 \\ \mathrm{H} & 8.22792000 & 7.77556000 & 0.33144200 \\ \mathrm{H} & 8.41352300 & 7.36433900 & 2.04391800 \\ \mathrm{H} & 7.75875900 & 8.94090900 & 1.58334200 \\ \mathrm{H} & 4.69132000 & 7.09740500 & 2.77244400 \\ \mathrm{H} & 6.29782800 & 6.95478200 & 3.50280600 \\ \mathrm{H} & 5.69079200 & 8.54287000 & 3.01396300 \\ \mathrm{H} & -4.58525700 & -7.58538900 & -0.13415800 \\ \mathrm{H} & -5.66620400 & -8.94971100 & -0.43442600 \\ \mathrm{H} & -6.03777400 & -7.81000900 & 0.86326100 \\ \mathrm{H} & -4.81068200 & -6.90498900 & -2.60234700 \\ \mathrm{H} & -6.41024500 & -6.73935200 & -3.34253600 \\ \mathrm{H} & -5.83792300 & -8.33302600 & -2.83048600 \\ \mathrm{H} & -8.54068200 & -7.08877700 & -1.88993900 \\ \mathrm{H} & -7.91983400 & -8.67160600 & -1.40450900 \\ \mathrm{H} & -8.37206100 & -7.48004400 & -0.17116800 \\ \mathrm{H} & -8.39022700 & 7.38897700 & 1.99731700 \\ \mathrm{H} & -7.73257200 & 8.96116600 & 1.52602100 \\ \mathrm{H} & -8.20311900 & 7.78786900 & 0.28209300 \\ \mathrm{H} & -5.85620000 & 8.07316900 & -0.73698400 \\ \mathrm{H} & -5.46767800 & 9.19658500 & 0.56993100 \\ \mathrm{H} & -4.41472100 & 7.81106800 & 0.26794400 \\ \mathrm{H} & -4.66890000 & 7.12064800 & 2.72954100 \\ \mathrm{H} & -5.66605400 & 8.56942400 & 2.96057700 \\ \mathrm{H} & -6.27603300 & 6.98576500 & 3.46001200 \\ \mathrm{H} & 1.23262900 & 3.27156500 & 0.98780300 \\ \mathrm{H} & -1.21848600 & 3.27299600 & 0.98653400 \\ \mathrm{H} & 2.48684200 & -3.47455400 & -1.05078500 \\ \mathrm{H} & & & \\ & & \end{array}$

Mulliken spin densities:

$\begin{array}{rlc}1 & \mathrm{C} & -0.005529 \\ 2 & \mathrm{C} & 0.004745 \\ 3 & \mathrm{C} & 0.003619 \\ 4 & \mathrm{C} & 0.020155 \\ 5 & \mathrm{C} & -0.012818 \\ 6 & \mathrm{C} & 0.012438 \\ 7 & \mathrm{C} & 0.019019 \\ 8 & \mathrm{C} & 0.010307 \\ 9 & \mathrm{C} & 0.034320 \\ 10 & \mathrm{C} & -0.028684 \\ 11 & \mathrm{C} & -0.012462 \\ 12 & \mathrm{C} & 0.033122\end{array}$




\begin{tabular}{|c|c|c|}
\hline 13 & $\mathrm{C}$ & -0.004027 \\
\hline 14 & $\mathrm{C}$ & 0.010309 \\
\hline & $\mathrm{C}$ & -0.006345 \\
\hline & $\mathrm{C}$ & 0.006239 \\
\hline & $\mathrm{C}$ & 0.066394 \\
\hline 18 & $\mathrm{C}$ & -0.017447 \\
\hline & $\mathrm{C}$ & -0.041603 \\
\hline & $\mathrm{C}$ & 0.105471 \\
\hline 21 & $\mathrm{C}$ & 0.074587 \\
\hline & $\mathrm{C}$ & -0.024495 \\
\hline & $\mathrm{C}$ & -0.035223 \\
\hline 24 & $\mathrm{C}$ & 0.131282 \\
\hline 25 & $\mathrm{C}$ & 0.009967 \\
\hline 0 & $\mathrm{C}$ & 0.009848 \\
\hline 27 & $\mathrm{C}$ & 0.027355 \\
\hline 28 & $\mathrm{C}$ & -0.012716 \\
\hline & $\mathrm{C}$ & -0.012521 \\
\hline 3( & $\mathrm{C}$ & 0.027184 \\
\hline 31 & $\mathrm{C}$ & 0.096273 \\
\hline 32 & $\mathrm{C}$ & -0.017906 \\
\hline 33 & $\mathrm{C}$ & -0.017728 \\
\hline 34 & $\mathrm{C}$ & 0.095863 \\
\hline 35 & $\mathrm{C}$ & -0.035027 \\
\hline 36 & $\mathrm{C}$ & -0.024535 \\
\hline 3 & $\mathrm{C}$ & 0.074783 \\
\hline 38 & $\mathrm{C}$ & -0.017655 \\
\hline 35 & $\mathrm{C}$ & -0.041601 \\
\hline 40 & $\mathrm{C}$ & 0.131420 \\
\hline 41 & $\mathrm{C}$ & 0.066754 \\
\hline 42 & $\mathrm{C}$ & 0.034086 \\
\hline 43 & $\mathrm{C}$ & -0.028484 \\
\hline 44 & $\mathrm{C}$ & 0.105622 \\
\hline 45 & $\mathrm{C}$ & 0.010353 \\
\hline 46 & $\mathrm{C}$ & 0.018963 \\
\hline 47 & $\mathrm{C}$ & 0.003682 \\
\hline 48 & $\mathrm{C}$ & 0.020119 \\
\hline 49 & $\mathrm{C}$ & 0.004696 \\
\hline 50 & $\mathrm{C}$ & -0.005450 \\
\hline 51 & $\mathrm{C}$ & 0.012424 \\
\hline 52 & $\mathrm{C}$ & -0.012792 \\
\hline 53 & $\mathrm{C}$ & 0.010204 \\
\hline 54 & $\mathrm{C}$ & -0.003964 \\
\hline 55 & $\mathrm{C}$ & 0.032966 \\
\hline & $\mathrm{C}$ & -0.012409 \\
\hline
\end{tabular}




\begin{tabular}{|c|c|c|}
\hline 57 & $\mathrm{C}$ & 0.006194 \\
\hline 58 & $\mathrm{C}$ & -0.006317 \\
\hline 59 & $\mathrm{C}$ & -0.008280 \\
\hline 60 & $\mathrm{C}$ & -0.000735 \\
\hline 61 & $\mathrm{C}$ & 0.000473 \\
\hline 62 & $\mathrm{C}$ & 0.000005 \\
\hline 63 & $\mathrm{C}$ & 0.000299 \\
\hline 64 & $\mathrm{C}$ & 0.000105 \\
\hline 65 & $\mathrm{C}$ & -0.000036 \\
\hline 66 & $\mathrm{C}$ & 0.000080 \\
\hline 67 & $\mathrm{C}$ & 0.000221 \\
\hline 68 & $\mathrm{C}$ & -0.003788 \\
\hline 69 & $\mathrm{C}$ & -0.008211 \\
\hline 70 & $\mathrm{C}$ & -0.003813 \\
\hline 71 & $\mathrm{C}$ & -0.000735 \\
\hline 72 & $\mathrm{C}$ & 0.000465 \\
\hline 73 & $\mathrm{C}$ & 0.000311 \\
\hline 74 & $\mathrm{C}$ & 0.000003 \\
\hline 75 & $\mathrm{C}$ & 0.000102 \\
\hline 76 & $\mathrm{C}$ & -0.000036 \\
\hline 77 & $\mathrm{C}$ & 0.000222 \\
\hline 78 & $\mathrm{C}$ & 0.000081 \\
\hline 79 & $\mathrm{C}$ & 0.007747 \\
\hline 80 & $\mathrm{C}$ & -0.003824 \\
\hline 81 & $\mathrm{C}$ & 0.007871 \\
\hline 82 & $\mathrm{C}$ & -0.002500 \\
\hline 83 & $\mathrm{C}$ & 0.007021 \\
\hline 84 & $\mathrm{C}$ & 0.006740 \\
\hline 85 & $\mathrm{C}$ & -0.003366 \\
\hline 86 & $\mathrm{C}$ & 0.007702 \\
\hline 87 & $\mathrm{C}$ & -0.002597 \\
\hline 88 & $\mathrm{C}$ & 0.005833 \\
\hline 89 & $\mathrm{C}$ & 0.005873 \\
\hline 90 & $\mathrm{C}$ & -0.002617 \\
\hline 91 & $\mathrm{C}$ & 0.007765 \\
\hline 92 & $\mathrm{C}$ & -0.003396 \\
\hline 93 & $\mathrm{C}$ & 0.006795 \\
\hline 94 & $\mathrm{C}$ & 0.006520 \\
\hline 95 & $\mathrm{C}$ & -0.002460 \\
\hline 96 & $\mathrm{C}$ & 0.008097 \\
\hline 97 & $\mathrm{C}$ & -0.003927 \\
\hline 98 & $\mathrm{C}$ & 0.008501 \\
\hline 99 & $\mathrm{C}$ & -0.000480 \\
\hline 100 & $\mathrm{C}$ & 0.000020 \\
\hline
\end{tabular}




\begin{tabular}{|c|c|c|}
\hline 101 & $\mathrm{C}$ & 0.000256 \\
\hline 102 & $\mathrm{C}$ & 0.000248 \\
\hline 103 & $\mathrm{C}$ & -0.000467 \\
\hline 04 & $\mathrm{C}$ & 0.000014 \\
\hline 105 & $\mathrm{C}$ & 0.000232 \\
\hline 106 & $\mathrm{C}$ & 0.000282 \\
\hline 107 & $\mathrm{C}$ & -0.000501 \\
\hline 108 & $\mathrm{C}$ & 0.000014 \\
\hline 109 & $\mathrm{C}$ & 0.000284 \\
\hline 110 & $\mathrm{C}$ & 0.000238 \\
\hline 111 & $\mathrm{C}$ & -0.000471 \\
\hline 112 & $\mathrm{C}$ & 0.000234 \\
\hline 113 & $\mathrm{C}$ & 0.000014 \\
\hline 114 & $\mathrm{C}$ & 0.000284 \\
\hline 115 & $\mathrm{C}$ & 0.035076 \\
\hline 116 & $\mathrm{C}$ & 0.010566 \\
\hline 117 & $\mathrm{C}$ & 0.010919 \\
\hline 118 & $\mathrm{C}$ & 0.034765 \\
\hline 119 & $\mathrm{H}$ & 0.000227 \\
\hline 120 & $\mathrm{H}$ & 0.000418 \\
\hline 121 & $\mathrm{H}$ & -0.001438 \\
\hline 122 & $\mathrm{H}$ & -0.000616 \\
\hline 123 & $\mathrm{H}$ & 0.000252 \\
\hline 124 & $\mathrm{H}$ & -0.000264 \\
\hline 125 & $\mathrm{H}$ & -0.002861 \\
\hline 126 & $\mathrm{H}$ & -0.005156 \\
\hline 127 & $\mathrm{H}$ & -0.000671 \\
\hline 128 & $\mathrm{H}$ & -0.000665 \\
\hline 129 & $\mathrm{H}$ & -0.001187 \\
\hline 130 & $\mathrm{H}$ & -0.002867 \\
\hline 131 & $\mathrm{H}$ & -0.005161 \\
\hline 132 & $\mathrm{H}$ & 0.000223 \\
\hline 133 & $\mathrm{H}$ & 0.000414 \\
\hline 134 & $\mathrm{H}$ & -0.000611 \\
\hline 135 & $\mathrm{H}$ & -0.001432 \\
\hline 136 & $\mathrm{H}$ & -0.000262 \\
\hline 137 & $\mathrm{H}$ & 0.000251 \\
\hline 138 & $\mathrm{H}$ & -0.000020 \\
\hline 139 & $\mathrm{H}$ & -0.000017 \\
\hline 140 & $\mathrm{H}$ & 0.000200 \\
\hline 141 & $\mathrm{H}$ & -0.000009 \\
\hline 142 & $\mathrm{H}$ & 0.000015 \\
\hline 143 & $\mathrm{H}$ & -0.000012 \\
\hline 144 & $\mathrm{H}$ & -0.000013 \\
\hline
\end{tabular}




$\begin{array}{ccc}145 & \mathrm{H} & 0.000003 \\ 146 & \mathrm{H} & 0.000128 \\ 147 & \mathrm{H} & -0.000012 \\ 148 & \mathrm{H} & -0.000005 \\ 149 & \mathrm{H} & 0.000011 \\ 150 & \mathrm{H} & -0.000005 \\ 151 & \mathrm{H} & 0.000152 \\ 152 & \mathrm{H} & 0.000013 \\ 153 & \mathrm{H} & 0.000007 \\ 154 & \mathrm{H} & 0.000202 \\ 155 & \mathrm{H} & -0.000028 \\ 156 & \mathrm{H} & -0.000017 \\ 157 & \mathrm{H} & 0.000196 \\ 158 & \mathrm{H} & -0.000017 \\ 159 & \mathrm{H} & 0.000003 \\ 160 & \mathrm{H} & -0.000013 \\ 161 & \mathrm{H} & 0.000133 \\ 162 & \mathrm{H} & 0.000015 \\ 163 & \mathrm{H} & -0.000012 \\ 164 & \mathrm{H} & -0.000012 \\ 165 & \mathrm{H} & 0.000011 \\ 166 & \mathrm{H} & -0.000005 \\ 167 & \mathrm{H} & -0.000012 \\ 168 & \mathrm{H} & 0.000007 \\ 169 & \mathrm{H} & -0.000028 \\ 170 & \mathrm{H} & 0.000202 \\ 171 & \mathrm{H} & -0.000005 \\ 172 & \mathrm{H} & 0.000151 \\ 173 & \mathrm{H} & 0.000013 \\ 174 & \mathrm{H} & -0.000456 \\ 175 & \mathrm{H} & 0.000336 \\ 176 & \mathrm{H} & 0.000209 \\ 177 & \mathrm{H} & -0.000258 \\ 178 & \mathrm{H} & -0.000385 \\ 179 & \mathrm{H} & 0.000263 \\ 180 & \mathrm{H} & 0.000168 \\ 181 & \mathrm{H} & -0.000224 \\ 182 & \mathrm{H} & -0.000226 \\ 183 & \mathrm{H} & 0.000169 \\ & \mathrm{H} & 0.000265 \\ & & -0.000387 \\ 184 & 0.000235 \\ 18000328\end{array}$




$\begin{array}{ccc}189 & \mathrm{H} & -0.000494 \\ 190 & \mathrm{H} & 0.000004 \\ 191 & \mathrm{H} & -0.000008 \\ 192 & \mathrm{H} & 0.000004 \\ 193 & \mathrm{H} & -0.000004 \\ 194 & \mathrm{H} & 0.000104 \\ 195 & \mathrm{H} & -0.000004 \\ 196 & \mathrm{H} & -0.000004 \\ 197 & \mathrm{H} & -0.000014 \\ 198 & \mathrm{H} & 0.000046 \\ 199 & \mathrm{H} & 0.000006 \\ 200 & \mathrm{H} & 0.000003 \\ 201 & \mathrm{H} & -0.000001 \\ 202 & \mathrm{H} & -0.000003 \\ 203 & \mathrm{H} & -0.000010 \\ 204 & \mathrm{H} & 0.000077 \\ 205 & \mathrm{H} & -0.000004 \\ 206 & \mathrm{H} & -0.000013 \\ 207 & \mathrm{H} & 0.000090 \\ 208 & \mathrm{H} & 0.000006 \\ 209 & \mathrm{H} & -0.000003 \\ 210 & \mathrm{H} & 0.000006 \\ 211 & \mathrm{H} & -0.000006 \\ 212 & \mathrm{H} & -0.000013 \\ 213 & \mathrm{H} & 0.000092 \\ 214 & \mathrm{H} & -0.000009 \\ 215 & \mathrm{H} & 0.000090 \\ 216 & \mathrm{H} & -0.000001 \\ 217 & \mathrm{H} & -0.000010 \\ 218 & \mathrm{H} & 0.000078 \\ 219 & \mathrm{H} & -0.000003 \\ 220 & \mathrm{H} & 0.000006 \\ 221 & \mathrm{H} & -0.000001 \\ 222 & \mathrm{H} & 0.000004 \\ 223 & \mathrm{H} & -0.000004 \\ 224 & \mathrm{H} & 0.000091 \\ 225 & \mathrm{H} & -0.000013 \\ 226 & \mathrm{H} & -0.000771 \\ 228 & \mathrm{H} & -0.000785 \\ & & -0.001195\end{array}$

\section{$\operatorname{OS} 9 \mathbf{a}^{2 \cdot+}$}

Energy (with Zero Point Energy correction $)=-4562.575492(-4560.635209)$ Hartree Free energy $(298 \mathrm{~K})=-4560.781676$ Hartree

Zero number of imaginary frequency 


\begin{tabular}{|c|c|c|c|}
\hline $\mathrm{C}$ & 11.48334200 & -2.38481500 & 1.22523600 \\
\hline $\mathrm{C}$ & 11.47153800 & -1.14112200 & 0.57837200 \\
\hline $\mathrm{C}$ & 10.21841900 & -0.52626400 & 0.26828400 \\
\hline $\mathrm{C}$ & 9.00736100 & -1.22651100 & 0.52907200 \\
\hline $\mathrm{C}$ & 9.08338400 & -2.44318900 & 1.21796300 \\
\hline $\mathrm{C}$ & 10.30475500 & -3.03512000 & 1.59320400 \\
\hline $\mathrm{C}$ & 10.19462000 & 0.77249000 & -0.30872100 \\
\hline $\mathrm{C}$ & 8.96032200 & 1.47488500 & -0.48364500 \\
\hline $\mathrm{C}$ & 7.72680700 & 0.84319100 & -0.04768800 \\
\hline $\mathrm{C}$ & 7.73271100 & -0.58486300 & 0.15983400 \\
\hline $\mathrm{C}$ & 11.41570800 & 1.40265600 & -0.68163500 \\
\hline $\mathrm{C}$ & 11.37572600 & 2.64973600 & -1.31928900 \\
\hline $\mathrm{C}$ & 10.16803200 & 3.30553400 & -1.62099600 \\
\hline $\mathrm{C}$ & 8.98738900 & 2.71301400 & -1.17851800 \\
\hline $\mathrm{C}$ & 12.68522800 & -0.46622700 & 0.22199000 \\
\hline $\mathrm{C}$ & 12.66027800 & 0.74516200 & -0.40032900 \\
\hline $\mathrm{C}$ & 6.49941000 & 1.56921000 & 0.13764900 \\
\hline $\mathrm{C}$ & 5.27643400 & 0.85698500 & 0.16117100 \\
\hline $\mathrm{C}$ & 5.28523900 & -0.56062000 & -0.09205400 \\
\hline $\mathrm{C}$ & 6.52315500 & -1.28592400 & -0.02929700 \\
\hline $\mathrm{C}$ & 4.01681000 & 1.50251200 & 0.40026000 \\
\hline $\mathrm{C}$ & 2.83799500 & 0.83035700 & 0.21943500 \\
\hline $\mathrm{C}$ & 2.84337100 & -0.56202800 & -0.21495800 \\
\hline $\mathrm{C}$ & 4.05665600 & -1.22039000 & -0.34727400 \\
\hline $\mathrm{C}$ & -0.70017800 & -4.50846500 & -1.51560600 \\
\hline $\mathrm{C}$ & 0.70852100 & -4.50991000 & -1.51287500 \\
\hline $\mathrm{C}$ & 1.40363500 & -3.37644100 & -1.14222100 \\
\hline $\mathrm{C}$ & 0.74073100 & -2.18625900 & -0.75377600 \\
\hline $\mathrm{C}$ & -0.73135500 & -2.18542500 & -0.75470200 \\
\hline $\mathrm{C}$ & -1.39486800 & -3.37423100 & -1.14632800 \\
\hline $\mathrm{C}$ & 1.45755100 & -1.00142000 & -0.37145500 \\
\hline $\mathrm{C}$ & 0.69200000 & 0.12003100 & -0.01576500 \\
\hline $\mathrm{C}$ & -0.68069200 & 0.12063500 & -0.01614300 \\
\hline $\mathrm{C}$ & -1.44735500 & -1.00021800 & -0.37168900 \\
\hline $\mathrm{C}$ & -2.83285800 & -0.55973700 & -0.21431700 \\
\hline $\mathrm{C}$ & -2.82577600 & 0.83359000 & 0.21715400 \\
\hline $\mathrm{C}$ & -4.00365400 & 1.50825000 & 0.39462300 \\
\hline $\mathrm{C}$ & -5.26379700 & 0.86359600 & 0.15712300 \\
\hline $\mathrm{C}$ & -5.27469700 & -0.55550400 & -0.08773100 \\
\hline $\mathrm{C}$ & -4.04713400 & -1.21779900 & -0.34176700 \\
\hline $\mathrm{C}$ & -6.48536700 & 1.57785100 & 0.12790800 \\
\hline $\mathrm{C}$ & -7.71391400 & 0.85280600 & -0.05259600 \\
\hline $\mathrm{C}$ & -7.72245600 & -0.57339800 & 0.16616500 \\
\hline
\end{tabular}




\begin{tabular}{|c|c|c|c|}
\hline $\mathrm{C}$ & -6.51389900 & -1.27821500 & -0.01826100 \\
\hline $\mathrm{C}$ & -8.94622600 & 1.48398500 & -0.49249400 \\
\hline $\mathrm{C}$ & -10.18196800 & 0.78651500 & -0.30925500 \\
\hline $\mathrm{C}$ & -10.20790900 & -0.50694500 & 0.27943100 \\
\hline $\mathrm{C}$ & -8.99814800 & -1.20879100 & 0.54305500 \\
\hline $\mathrm{C}$ & -11.46216300 & -1.11466700 & 0.59884600 \\
\hline $\mathrm{C}$ & -11.47617700 & -2.35167800 & 1.25805700 \\
\hline $\mathrm{C}$ & -10.29863500 & -3.00309000 & 1.62767700 \\
\hline $\mathrm{C}$ & -9.07639800 & -2.41959800 & 1.24239700 \\
\hline $\mathrm{C}$ & -8.97087300 & 2.71604600 & -1.19785900 \\
\hline $\mathrm{C}$ & -10.15067000 & 3.30783500 & -1.64365000 \\
\hline $\mathrm{C}$ & -11.35965700 & 2.65808600 & -1.33408500 \\
\hline $\mathrm{C}$ & -11.40202200 & 1.41693600 & -0.68523900 \\
\hline $\mathrm{C}$ & -12.64788700 & 0.76577000 & -0.39483600 \\
\hline $\mathrm{C}$ & -12.67480500 & -0.43937500 & 0.23922900 \\
\hline $\mathrm{C}$ & 6.49689300 & -2.75810600 & -0.26559800 \\
\hline $\mathrm{C}$ & 10.37371300 & -4.36628500 & 2.36387300 \\
\hline $\mathrm{C}$ & 11.01141900 & -5.43886400 & 1.44703100 \\
\hline $\mathrm{C}$ & 8.98523700 & -4.86577100 & 2.80557300 \\
\hline $\mathrm{C}$ & 11.24314200 & -4.18986300 & 3.63227300 \\
\hline $\mathrm{C}$ & 10.20025200 & 4.62818600 & -2.40615500 \\
\hline $\mathrm{C}$ & 8.78942000 & 5.15573800 & -2.72462500 \\
\hline $\mathrm{C}$ & 10.94793100 & 4.41494100 & -3.74498200 \\
\hline $\mathrm{C}$ & 10.94362500 & 5.69367100 & -1.56386800 \\
\hline $\mathrm{C}$ & 6.51208100 & 3.02824000 & 0.41017900 \\
\hline $\mathrm{C}$ & -6.49203400 & -2.75226400 & -0.24037200 \\
\hline $\mathrm{C}$ & -6.49540700 & 3.03883800 & 0.38964600 \\
\hline $\mathrm{C}$ & -10.37135000 & -4.32322300 & 2.41678600 \\
\hline $\mathrm{C}$ & -11.05052300 & -5.39790900 & 1.53295400 \\
\hline $\mathrm{C}$ & -11.20604500 & -4.11170500 & 3.70324000 \\
\hline $\mathrm{C}$ & -8.98155600 & -4.84188300 & 2.83104500 \\
\hline $\mathrm{C}$ & -10.18051100 & 4.62374100 & -2.44014500 \\
\hline $\mathrm{C}$ & -8.76873500 & 5.14452200 & -2.76559100 \\
\hline $\mathrm{C}$ & -10.91935800 & 5.69857900 & -1.60579000 \\
\hline $\mathrm{C}$ & -10.93114600 & 4.40115300 & -3.77579000 \\
\hline $\mathrm{C}$ & 5.75620600 & -3.63713700 & 0.54526100 \\
\hline $\mathrm{C}$ & 5.72253400 & -5.00215700 & 0.27280000 \\
\hline $\mathrm{C}$ & 6.40832100 & -5.55760200 & -0.82300500 \\
\hline $\mathrm{C}$ & 7.14069300 & -4.67058000 & -1.62703100 \\
\hline $\mathrm{C}$ & 7.19048800 & -3.30396300 & -1.35474000 \\
\hline $\mathrm{C}$ & 5.72517900 & 3.94161400 & -0.31242900 \\
\hline $\mathrm{C}$ & 5.73394700 & 5.30154400 & -0.00257200 \\
\hline $\mathrm{C}$ & 6.50847200 & 5.81288000 & 1.05094100 \\
\hline $\mathrm{C}$ & 7.29084400 & 4.88942300 & 1.77138100 \\
\hline
\end{tabular}




\begin{tabular}{|c|c|c|c|}
\hline $\mathrm{C}$ & 7.30567500 & 3.53710800 & 1.45763800 \\
\hline $\mathrm{C}$ & -7.28898200 & 3.55726800 & 1.43242200 \\
\hline $\mathrm{C}$ & -7.27185800 & 4.91192300 & 1.73570400 \\
\hline $\mathrm{C}$ & -6.48719000 & 5.82833000 & 1.00876300 \\
\hline $\mathrm{C}$ & -5.71255800 & 5.30739100 & -0.03995000 \\
\hline $\mathrm{C}$ & -5.70596500 & 3.94506700 & -0.33916300 \\
\hline $\mathrm{C}$ & -7.19929500 & -3.30810700 & -1.32059000 \\
\hline $\mathrm{C}$ & -7.16013400 & -4.67455200 & -1.57028600 \\
\hline $\mathrm{C}$ & -6.42957900 & -5.56052400 & -0.75536000 \\
\hline $\mathrm{C}$ & -5.73103400 & -4.99813000 & 0.32293300 \\
\hline $\mathrm{C}$ & -5.75294400 & -3.62403700 & 0.57349700 \\
\hline $\mathrm{C}$ & 6.32895900 & -7.06876300 & -1.09962300 \\
\hline $\mathrm{C}$ & 7.15428300 & -7.48217300 & -2.33275800 \\
\hline $\mathrm{C}$ & 4.85250000 & -7.46217400 & -1.34963700 \\
\hline $\mathrm{C}$ & 6.86501900 & -7.84667500 & 0.12629500 \\
\hline $\mathrm{C}$ & 6.52653000 & 7.30165900 & 1.43203500 \\
\hline $\mathrm{C}$ & 5.62291500 & 8.15120700 & 0.51862800 \\
\hline $\mathrm{C}$ & 7.97317600 & 7.84230000 & 1.32352200 \\
\hline $\mathrm{C}$ & 6.02939800 & 7.45723200 & 2.89040100 \\
\hline $\mathrm{C}$ & -6.41812700 & -7.06605200 & -1.06935500 \\
\hline $\mathrm{C}$ & -5.58690900 & -7.86974700 & -0.05166400 \\
\hline $\mathrm{C}$ & -5.81168500 & -7.28710800 & -2.47653000 \\
\hline $\mathrm{C}$ & -7.86787500 & -7.60788600 & -1.04653100 \\
\hline $\mathrm{C}$ & -6.50297700 & 7.32007300 & 1.37817500 \\
\hline $\mathrm{C}$ & -7.94862400 & 7.86232600 & 1.26449100 \\
\hline $\mathrm{C}$ & -5.59733000 & 8.16091700 & 0.45875000 \\
\hline $\mathrm{C}$ & -6.00654900 & 7.48622100 & 2.83561900 \\
\hline $\mathrm{C}$ & 1.43755700 & 1.25845800 & 0.35772200 \\
\hline $\mathrm{C}$ & 0.72203000 & 2.39738400 & 0.72644600 \\
\hline $\mathrm{C}$ & -0.70859800 & 2.39826600 & 0.72547000 \\
\hline $\mathrm{C}$ & -1.42510500 & 1.26011600 & 0.35617400 \\
\hline $\mathrm{H}$ & 12.44697800 & -2.82912200 & 1.45590700 \\
\hline $\mathrm{H}$ & 8.16637700 & -2.94128500 & 1.49097000 \\
\hline $\mathrm{H}$ & 12.32055700 & 3.10178900 & -1.60714500 \\
\hline $\mathrm{H}$ & 8.05002500 & 3.19579600 & -1.40273900 \\
\hline $\mathrm{H}$ & 13.63143800 & -0.94858600 & 0.45077800 \\
\hline $\mathrm{H}$ & 13.58451500 & 1.24214400 & -0.68125200 \\
\hline $\mathrm{H}$ & 4.00872000 & 2.53727900 & 0.71974800 \\
\hline $\mathrm{H}$ & 4.08867700 & -2.26253300 & -0.63059500 \\
\hline $\mathrm{H}$ & -1.24179900 & -5.40150700 & -1.81213300 \\
\hline $\mathrm{H}$ & 1.25009400 & -5.40414300 & -1.80596300 \\
\hline $\mathrm{H}$ & -2.47796300 & -3.39356400 & -1.16065500 \\
\hline $\mathrm{H}$ & -3.99434900 & 2.54422400 & 0.71019400 \\
\hline $\mathrm{H}$ & -4.08075800 & -2.26148800 & -0.61947300 \\
\hline
\end{tabular}




\begin{tabular}{|c|c|c|c|}
\hline $\mathrm{H}$ & -12.44057800 & -2.78978800 & 1.49741400 \\
\hline $\mathrm{H}$ & -8.16034000 & -2.91884500 & 1.51639500 \\
\hline $\mathrm{H}$ & -8.03249000 & 3.19433500 & -1.42752800 \\
\hline $\mathrm{H}$ & -12.30373000 & 3.11033000 & -1.62411900 \\
\hline $\mathrm{H}$ & -13.57134100 & 1.26280000 & -0.67824300 \\
\hline $\mathrm{H}$ & -13.62182300 & -0.91668300 & 0.47516300 \\
\hline $\mathrm{H}$ & 10.40987400 & -5.59159100 & 0.54353500 \\
\hline $\mathrm{H}$ & 12.02284300 & -5.15943200 & 1.13371300 \\
\hline $\mathrm{H}$ & 11.08003600 & -6.39560200 & 1.97737400 \\
\hline $\mathrm{H}$ & 8.33039300 & -5.07754500 & 1.95352400 \\
\hline $\mathrm{H}$ & 8.48097100 & -4.14367000 & 3.45863900 \\
\hline $\mathrm{H}$ & 9.09736300 & -5.79642400 & 3.37164700 \\
\hline $\mathrm{H}$ & 12.27001200 & -3.89440600 & 3.39525500 \\
\hline $\mathrm{H}$ & 10.81969500 & -3.43046800 & 4.29921200 \\
\hline $\mathrm{H}$ & 11.29352100 & -5.13484300 & 4.18450400 \\
\hline $\mathrm{H}$ & 8.21619800 & 5.37556600 & -1.81743800 \\
\hline $\mathrm{H}$ & 8.86977700 & 6.08585700 & -3.29679200 \\
\hline $\mathrm{H}$ & 8.21609300 & 4.44622100 & -3.33298100 \\
\hline $\mathrm{H}$ & 10.44577400 & 3.66218700 & -4.36298300 \\
\hline $\mathrm{H}$ & 10.97554700 & 5.35306500 & -4.31018100 \\
\hline $\mathrm{H}$ & 11.98353900 & 4.09226600 & -3.59663400 \\
\hline $\mathrm{H}$ & 11.97198800 & 5.39430500 & -1.33506800 \\
\hline $\mathrm{H}$ & 10.99109000 & 6.64029300 & -2.11392300 \\
\hline $\mathrm{H}$ & 10.42716800 & 5.87630300 & -0.61468000 \\
\hline $\mathrm{H}$ & -10.47415700 & -5.57706200 & 0.61810900 \\
\hline $\mathrm{H}$ & -11.12352100 & -6.34440100 & 2.08077900 \\
\hline $\mathrm{H}$ & -12.06431600 & -5.10591700 & 1.23965100 \\
\hline $\mathrm{H}$ & -10.75158100 & -3.35167700 & 4.34872200 \\
\hline $\mathrm{H}$ & -12.23186800 & -3.79810500 & 3.48552900 \\
\hline $\mathrm{H}$ & -11.26223000 & -5.04758900 & 4.27028800 \\
\hline $\mathrm{H}$ & -8.45076600 & -4.12401900 & 3.46750300 \\
\hline $\mathrm{H}$ & -9.09598100 & -5.76652100 & 3.40651800 \\
\hline $\mathrm{H}$ & -8.34973100 & -5.06923700 & 1.96561400 \\
\hline $\mathrm{H}$ & -8.19843400 & 4.42809500 & -3.36866900 \\
\hline $\mathrm{H}$ & -8.84746000 & 6.06982500 & -3.34573600 \\
\hline $\mathrm{H}$ & -8.19334600 & 5.37068900 & -1.86132800 \\
\hline $\mathrm{H}$ & -11.94821900 & 5.40421900 & -1.37279200 \\
\hline $\mathrm{H}$ & -10.40081600 & 5.88773600 & -0.65901900 \\
\hline $\mathrm{H}$ & -10.96492300 & 6.64062100 & -2.16381500 \\
\hline $\mathrm{H}$ & -10.43216000 & 3.64176300 & -4.38821900 \\
\hline $\mathrm{H}$ & -10.95718800 & 5.33448900 & -4.34893900 \\
\hline $\mathrm{H}$ & -11.96736400 & 4.08260500 & -3.62285200 \\
\hline $\mathrm{H}$ & 5.22364300 & -3.25172800 & 1.41100100 \\
\hline $\mathrm{H}$ & 5.15045800 & -5.64583100 & 0.93457500 \\
\hline
\end{tabular}




\begin{tabular}{|c|c|c|c|}
\hline $\mathrm{H}$ & 7.68377000 & -5.04010700 & -2.48918900 \\
\hline $\mathrm{H}$ & 7.76729800 & -2.65157200 & -2.00393700 \\
\hline $\mathrm{H}$ & 5.12922700 & 3.59179600 & -1.15104000 \\
\hline $\mathrm{H}$ & 5.12609500 & 5.96810500 & -0.60354700 \\
\hline $\mathrm{H}$ & 7.90238600 & 5.23268300 & 2.60012900 \\
\hline $\mathrm{H}$ & 7.91905300 & 2.85826600 & 2.04250700 \\
\hline $\mathrm{H}$ & -7.90419400 & 2.88413200 & 2.02193500 \\
\hline $\mathrm{H}$ & -7.88344500 & 5.26273200 & 2.56124500 \\
\hline $\mathrm{H}$ & -5.10285100 & 5.96811900 & -0.64546900 \\
\hline $\mathrm{H}$ & -5.10971400 & 3.58757900 & -1.17431800 \\
\hline $\mathrm{H}$ & -7.77566200 & -2.65956300 & -1.97393700 \\
\hline $\mathrm{H}$ & -7.71108500 & -5.05778400 & -2.42388600 \\
\hline $\mathrm{H}$ & -5.15963700 & -5.63138900 & 0.99208300 \\
\hline $\mathrm{H}$ & -5.21120400 & -3.23166600 & 1.43038300 \\
\hline $\mathrm{H}$ & 6.80056300 & -6.99300400 & -3.24747000 \\
\hline $\mathrm{H}$ & 7.06878300 & -8.56294100 & -2.48573100 \\
\hline $\mathrm{H}$ & 8.21855800 & -7.25262900 & -2.20866500 \\
\hline $\mathrm{H}$ & 4.44728000 & -6.93328500 & -2.22045500 \\
\hline $\mathrm{H}$ & 4.77659300 & -8.53809500 & -1.54323600 \\
\hline $\mathrm{H}$ & 4.21677600 & -7.23528000 & -0.48670800 \\
\hline $\mathrm{H}$ & 7.91266900 & -7.59441300 & 0.32519300 \\
\hline $\mathrm{H}$ & 6.28951600 & -7.63450800 & 1.03350900 \\
\hline $\mathrm{H}$ & 6.80715200 & -8.92536500 & -0.05779500 \\
\hline $\mathrm{H}$ & 5.93933500 & 8.10569700 & -0.52972700 \\
\hline $\mathrm{H}$ & 4.57306600 & 7.84128000 & 0.57660300 \\
\hline $\mathrm{H}$ & 5.67122600 & 9.19988300 & 0.82921100 \\
\hline $\mathrm{H}$ & 8.35249700 & 7.75250800 & 0.29917600 \\
\hline $\mathrm{H}$ & 8.66255100 & 7.31044000 & 1.98742200 \\
\hline $\mathrm{H}$ & 7.99927900 & 8.90193800 & 1.60107800 \\
\hline $\mathrm{H}$ & 5.00294700 & 7.08879800 & 2.99920700 \\
\hline $\mathrm{H}$ & 6.65985800 & 6.91193400 & 3.60027500 \\
\hline $\mathrm{H}$ & 6.04211400 & 8.51399700 & 3.17967800 \\
\hline $\mathrm{H}$ & -4.53514500 & -7.56041800 & -0.04427500 \\
\hline $\mathrm{H}$ & -5.61297100 & -8.93229300 & -0.31443400 \\
\hline $\mathrm{H}$ & -5.98092800 & -7.77435500 & 0.96649600 \\
\hline $\mathrm{H}$ & -4.77948700 & -6.92050400 & -2.52343000 \\
\hline $\mathrm{H}$ & -6.38533200 & -6.77540900 & -3.25622300 \\
\hline $\mathrm{H}$ & -5.80208100 & -8.35569400 & -2.71912300 \\
\hline $\mathrm{H}$ & -8.50494200 & -7.11001200 & -1.78484600 \\
\hline $\mathrm{H}$ & -7.87282700 & -8.67951700 & -1.27534900 \\
\hline $\mathrm{H}$ & -8.32435300 & -7.47134900 & -0.05961800 \\
\hline $\mathrm{H}$ & -8.63931800 & 7.33699600 & 1.93220600 \\
\hline $\mathrm{H}$ & -7.97303300 & 8.92418800 & 1.53357800 \\
\hline $\mathrm{H}$ & -8.32751000 & 7.76502200 & 0.24067200 \\
\hline
\end{tabular}




$\begin{array}{lrrr}\mathrm{H} & -5.91308600 & 8.10764100 & -0.58943900 \\ \mathrm{H} & -5.64414500 & 9.21208900 & 0.76101400 \\ \mathrm{H} & -4.54802800 & 7.84975800 & 0.51992000 \\ \mathrm{H} & -4.98074600 & 7.11704700 & 2.94797100 \\ \mathrm{H} & -6.01780600 & 8.54522800 & 3.11664300 \\ \mathrm{H} & -6.63832700 & 6.94745100 & 3.54929800 \\ \mathrm{H} & 1.23222700 & 3.30815500 & 1.02707800 \\ \mathrm{H} & -1.21807300 & 3.30970000 & 1.02532600 \\ \mathrm{H} & 2.48669600 & -3.39815900 & -1.15201600\end{array}$

Mulliken spin densities:

$\begin{array}{rlc}1 & \mathrm{C} & 0.026016 \\ 2 & \mathrm{C} & -0.028530 \\ 3 & \mathrm{C} & -0.023602 \\ 4 & \mathrm{C} & -0.008252 \\ 5 & \mathrm{C} & -0.001561 \\ 6 & \mathrm{C} & -0.029114 \\ 7 & \mathrm{C} & -0.016920 \\ 8 & \mathrm{C} & -0.069802 \\ 9 & \mathrm{C} & -0.034024 \\ 10 & \mathrm{C} & 0.032075 \\ 11 & \mathrm{C} & 0.043624 \\ 12 & \mathrm{C} & -0.148436 \\ 13 & \mathrm{C} & 0.023975 \\ 14 & \mathrm{C} & -0.049639 \\ 15 & \mathrm{C} & 0.015418 \\ 16 & \mathrm{C} & -0.045464 \\ 17 & \mathrm{C} & -0.147446 \\ 18 & \mathrm{C} & 0.054602 \\ 19 & \mathrm{C} & -0.019701 \\ 20 & \mathrm{C} & -0.070758 \\ 21 & \mathrm{C} & -0.074948 \\ 22 & \mathrm{C} & 0.032610 \\ 23 & \mathrm{C} & -0.070473 \\ 24 & \mathrm{C} & 0.004041 \\ 25 & \mathrm{C} & 0.045765 \\ 26 & \mathrm{C} & -0.045730 \\ 27 & \mathrm{C} & 0.040939 \\ 28 & \mathrm{C} & -0.054181 \\ 29 & \mathrm{C} & 0.054200 \\ 30 & \mathrm{C} & -0.040919 \\ 31 & \mathrm{C} & 0.111006 \\ 32 & \mathrm{C} & -0.045376 \\ 33 & \mathrm{C} & 0.045234 \\ 34 & \mathrm{C} & -0.110885\end{array}$




$\begin{array}{llc}35 & \mathrm{C} & 0.070462 \\ 36 & \mathrm{C} & -0.032600 \\ 37 & \mathrm{C} & 0.075353 \\ 38 & \mathrm{C} & -0.054811 \\ 39 & \mathrm{C} & 0.019391 \\ 40 & \mathrm{C} & -0.003420 \\ 41 & \mathrm{C} & 0.147977 \\ 42 & \mathrm{C} & 0.033280 \\ 43 & \mathrm{C} & -0.032216 \\ 44 & \mathrm{C} & 0.071706 \\ 45 & \mathrm{C} & 0.069688 \\ 46 & \mathrm{C} & 0.017000 \\ 47 & \mathrm{C} & 0.023639 \\ 48 & \mathrm{C} & 0.008670 \\ 49 & \mathrm{C} & 0.028261 \\ 50 & \mathrm{C} & -0.026044 \\ 51 & \mathrm{C} & 0.029318 \\ 52 & \mathrm{C} & 0.000983 \\ 53 & \mathrm{C} & 0.048613 \\ 54 & \mathrm{C} & -0.023377 \\ 55 & \mathrm{C} & 0.146861 \\ 56 & \mathrm{C} & -0.043273 \\ 57 & \mathrm{C} & 0.044901 \\ 58 & \mathrm{C} & -0.015472 \\ 59 & \mathrm{C} & 0.005090 \\ 60 & \mathrm{C} & 0.001667 \\ 61 & \mathrm{C} & -0.001269 \\ 62 & \mathrm{C} & -0.000110 \\ 63 & \mathrm{C} & -0.000988 \\ 64 & \mathrm{C} & -0.001051 \\ 65 & \mathrm{C} & 0.000027 \\ 66 & \mathrm{C} & -0.000341 \\ 67 & \mathrm{C} & -0.000643 \\ 68 & \mathrm{C} & 0.001384 \\ 69 & \mathrm{C} & -0.005102 \\ 70 & \mathrm{C} & -0.001450 \\ 71 & \mathrm{C} & -0.001676 \\ 72 & \mathrm{C} & 0.001254 \\ 73 & \mathrm{C} & 0.001029 \\ 74 & \mathrm{C} & 0.000098 \\ 75 & \mathrm{C} & 0.001021 \\ 76 & \mathrm{C} & -0.000029 \\ 77 & \mathrm{C} & 0.000652 \\ 78 & \mathrm{C} & 0.000347\end{array}$




$\begin{array}{rlc}79 & \mathrm{C} & -0.005265 \\ 80 & \mathrm{C} & 0.002159 \\ 81 & \mathrm{C} & -0.005783 \\ 82 & \mathrm{C} & 0.001149 \\ 83 & \mathrm{C} & -0.004000 \\ 84 & \mathrm{C} & -0.020320 \\ 85 & \mathrm{C} & 0.008320 \\ 86 & \mathrm{C} & -0.033670 \\ 87 & \mathrm{C} & 0.013136 \\ 88 & \mathrm{C} & -0.023239 \\ 89 & \mathrm{C} & 0.023244 \\ 90 & \mathrm{C} & -0.013134 \\ 91 & \mathrm{C} & 0.033717 \\ 92 & \mathrm{C} & -0.008381 \\ 93 & \mathrm{C} & 0.020398 \\ 94 & \mathrm{C} & 0.003682 \\ 95 & \mathrm{C} & -0.001023 \\ 96 & \mathrm{C} & 0.006174 \\ 97 & \mathrm{C} & -0.002354 \\ 98 & \mathrm{C} & 0.006047 \\ 99 & \mathrm{C} & 0.000342 \\ 100 & \mathrm{C} & -0.000011 \\ 120 & \mathrm{H} & 0.000162 \\ 101 & \mathrm{C} & -0.000219 \\ 102 & \mathrm{C} & -0.000231 \\ 103 & \mathrm{C} & 0.001954 \\ 104 & \mathrm{C} & -0.000068 \\ 105 & \mathrm{C} & -0.001209 \\ 106 & \mathrm{C} & -0.001378 \\ 107 & \mathrm{C} & -0.000359 \\ 108 & \mathrm{C} & 0.000007 \\ 109 & \mathrm{C} & 0.000247 \\ 110 & \mathrm{C} & 0.000240 \\ 111 & \mathrm{C} & -0.001957 \\ 112 & \mathrm{C} & 0.001210 \\ 113 & \mathrm{C} & 0.000068 \\ 114 & \mathrm{C} & 0.001379 \\ & & 0.0052666\end{array}$




$\begin{array}{ccc}123 & \mathrm{H} & -0.000521 \\ 124 & \mathrm{H} & 0.001858 \\ 125 & \mathrm{H} & 0.002703 \\ 126 & \mathrm{H} & -0.000114 \\ 127 & \mathrm{H} & -0.001947 \\ 128 & \mathrm{H} & 0.001941 \\ 129 & \mathrm{H} & 0.001749 \\ 130 & \mathrm{H} & -0.002713 \\ 131 & \mathrm{H} & 0.000088 \\ 132 & \mathrm{H} & 0.000944 \\ 133 & \mathrm{H} & -0.000144 \\ 134 & \mathrm{H} & -0.002622 \\ 135 & \mathrm{H} & -0.006214 \\ 136 & \mathrm{H} & -0.001835 \\ 137 & \mathrm{H} & 0.000524 \\ 138 & \mathrm{H} & 0.000027 \\ 139 & \mathrm{H} & 0.000060 \\ 140 & \mathrm{H} & -0.000282 \\ 141 & \mathrm{H} & 0.000004 \\ 142 & \mathrm{H} & -0.000032 \\ 143 & \mathrm{H} & 0.000035 \\ 144 & \mathrm{H} & 0.000053 \\ 145 & \mathrm{H} & -0.000006 \\ 146 & \mathrm{H} & -0.000162 \\ 147 & \mathrm{H} & 0.000048 \\ 148 & \mathrm{H} & 0.000024 \\ 149 & \mathrm{H} & -0.000050 \\ 150 & \mathrm{H} & 0.000023 \\ 151 & \mathrm{H} & -0.000562 \\ 152 & \mathrm{H} & -0.000043 \\ 153 & \mathrm{H} & -0.000028 \\ 154 & \mathrm{H} & -0.000662 \\ 155 & \mathrm{H} & 0.000080 \\ 156 & \mathrm{H} & -0.000022 \\ 157 & \mathrm{H} & 0.000271 \\ 158 & \mathrm{H} & -0.000059 \\ 159 & \mathrm{H} & 0.000005 \\ 160 & \mathrm{H} & -0.000054 \\ 161 & \mathrm{H} & 0.000178 \\ 162 & \mathrm{H} & 0.000032 \\ 166 & \mathrm{H} & -0.000036 \\ & & -0.000009 \\ 163 & 0.000050 \\ 1500024\end{array}$




$\begin{array}{ccc}167 & \mathrm{H} & -0.000048 \\ 168 & \mathrm{H} & 0.000027 \\ 169 & \mathrm{H} & -0.000080 \\ 170 & \mathrm{H} & 0.000662 \\ 171 & \mathrm{H} & -0.000023 \\ 172 & \mathrm{H} & 0.000561 \\ 173 & \mathrm{H} & 0.000042 \\ 174 & \mathrm{H} & 0.000271 \\ 175 & \mathrm{H} & -0.000246 \\ 176 & \mathrm{H} & -0.000155 \\ 177 & \mathrm{H} & 0.000138 \\ 178 & \mathrm{H} & 0.001011 \\ 179 & \mathrm{H} & -0.000520 \\ 180 & \mathrm{H} & -0.000653 \\ 181 & \mathrm{H} & 0.001058 \\ 182 & \mathrm{H} & -0.001057 \\ 183 & \mathrm{H} & 0.000654 \\ 184 & \mathrm{H} & 0.000524 \\ 185 & \mathrm{H} & -0.001014 \\ 186 & \mathrm{H} & -0.000125 \\ 187 & \mathrm{H} & 0.000159 \\ 188 & \mathrm{H} & 0.000247 \\ 189 & \mathrm{H} & -0.000309 \\ 190 & \mathrm{H} & -0.000007 \\ 191 & \mathrm{H} & 0.000003 \\ 192 & \mathrm{H} & -0.000002 \\ 193 & \mathrm{H} & -0.000002 \\ 194 & \mathrm{H} & -0.000077 \\ 195 & \mathrm{H} & 0.000006 \\ 196 & \mathrm{H} & 0.000004 \\ 197 & \mathrm{H} & 0.000012 \\ 198 & \mathrm{H} & -0.000046 \\ 199 & \mathrm{H} & -0.000034 \\ 200 & \mathrm{H} & -0.000019 \\ 201 & \mathrm{H} & 0.000029 \\ 202 & \mathrm{H} & 0.000019 \\ 203 & \mathrm{H} & 0.000050 \\ 204 & \mathrm{H} & -0.000349 \\ 205 & \mathrm{H} & 0.000015 \\ & \mathrm{H} & 0.000060 \\ & \mathrm{H} & -0.000364 \\ 10.0000005 \\ 1000004\end{array}$




$\begin{array}{ccc}211 & \mathrm{H} & -0.000002 \\ 212 & \mathrm{H} & -0.000011 \\ 213 & \mathrm{H} & 0.000066 \\ 214 & \mathrm{H} & -0.000009 \\ 215 & \mathrm{H} & 0.000090 \\ 216 & \mathrm{H} & 0.000001 \\ 217 & \mathrm{H} & -0.000050 \\ 218 & \mathrm{H} & 0.000350 \\ 219 & \mathrm{H} & -0.000018 \\ 220 & \mathrm{H} & 0.000034 \\ 221 & \mathrm{H} & -0.000029 \\ 222 & \mathrm{H} & 0.000019 \\ 223 & \mathrm{H} & -0.000015 \\ 224 & \mathrm{H} & 0.000364 \\ 225 & \mathrm{H} & -0.000060 \\ 226 & \mathrm{H} & 0.002180 \\ 227 & \mathrm{H} & -0.002191 \\ 228 & \mathrm{H} & -0.001745\end{array}$

\section{CS 9a ${ }^{2+}$}

Energy (with Zero Point Energy correction $)=-4562.577224(-4560.638646)$ Hartree Free energy $(298 \mathrm{~K})=-4560.787012$ Hartree

Zero number of imaginary frequency

21

C

$\mathrm{C}$

11.47290900

$-2.38179000$

1.23962600

$11.46527000 \quad-1.14123700 \quad 0.59042800$

C

10.21430400

$-0.52553900$

0.27702700

C

9.00127000

$-1.22556500$

0.53694900

$\mathrm{C}$

9.07310400

$-2.44197800$

1.22906100

C

10.29162700

$-3.03173200$

1.60790200

C

10.19202000

0.77207400

$-0.30282100$

C

8.95828700

1.47459500

$-0.48428200$

C

7.72285900

0.84046700

$-0.05287300$

C

7.73036000

$-0.58429400$

0.16289100

C

11.41567300

1.40018400

$-0.67549200$

C

11.37782600

2.64429900

$-1.31488500$

C

10.17191700

3.30033400

$-1.61766700$

C

8.98796500

2.71016600

$-1.17593900$

C

12.68263400

$-0.46787100$

0.23458700

C

12.65996300

0.73985200

$-0.39000200$

C

6.49326600

1.56427500

0.13821700

C

5.27519900

0.85196700

0.17016300

C

5.28202100

$-0.56659500$

$-0.08443700$

C

6.51876200

$-1.29008300$

$-0.02575100$

C

4.01419100

1.49675900

0.41627600 


\begin{tabular}{|c|c|c|c|}
\hline $\mathrm{C}$ & 2.83542200 & 0.82723300 & 0.23412300 \\
\hline $\mathrm{C}$ & 2.83853300 & -0.56433200 & -0.21063500 \\
\hline $\mathrm{C}$ & 4.05498600 & -1.22238500 & -0.34686700 \\
\hline $\mathrm{C}$ & -0.69810800 & -4.50425500 & -1.53465000 \\
\hline $\mathrm{C}$ & 0.70718500 & -4.50578200 & -1.53159700 \\
\hline $\mathrm{C}$ & 1.40215300 & -3.37132300 & -1.15293700 \\
\hline $\mathrm{C}$ & 0.73946100 & -2.18660400 & -0.75826200 \\
\hline $\mathrm{C}$ & -0.72954800 & -2.18569200 & -0.75951800 \\
\hline $\mathrm{C}$ & -1.39274500 & -3.36895500 & -1.15771300 \\
\hline $\mathrm{C}$ & 1.46126500 & -1.00037200 & -0.36841100 \\
\hline $\mathrm{C}$ & 0.69181800 & 0.12059800 & -0.00496800 \\
\hline $\mathrm{C}$ & -0.68002600 & 0.12125600 & -0.00558900 \\
\hline $\mathrm{C}$ & -1.45058400 & -0.99904600 & -0.36916300 \\
\hline $\mathrm{C}$ & -2.82755400 & -0.56187000 & -0.21078200 \\
\hline $\mathrm{C}$ & -2.82270100 & 0.83067100 & 0.23120500 \\
\hline $\mathrm{C}$ & -4.00045100 & 1.50282700 & 0.40981500 \\
\hline $\mathrm{C}$ & -5.26201300 & 0.85897300 & 0.16522400 \\
\hline $\mathrm{C}$ & -5.27103200 & -0.56112700 & -0.08101100 \\
\hline $\mathrm{C}$ & -4.04505500 & -1.21952900 & -0.34242300 \\
\hline $\mathrm{C}$ & -6.47857000 & 1.57336300 & 0.12768300 \\
\hline $\mathrm{C}$ & -7.70942300 & 0.85052800 & -0.05796500 \\
\hline $\mathrm{C}$ & -7.71948400 & -0.57229100 & 0.16917000 \\
\hline $\mathrm{C}$ & -6.50909100 & -1.28196700 & -0.01542000 \\
\hline $\mathrm{C}$ & -8.94380400 & 1.48402900 & -0.49299300 \\
\hline $\mathrm{C}$ & -10.17887600 & 0.78655500 & -0.30252900 \\
\hline $\mathrm{C}$ & -10.20304000 & -0.50554100 & 0.28943900 \\
\hline $\mathrm{C}$ & -8.99122000 & -1.20706700 & 0.55180400 \\
\hline $\mathrm{C}$ & -11.45495700 & -1.11397000 & 0.61300600 \\
\hline $\mathrm{C}$ & -11.46445400 & -2.34760000 & 1.27509100 \\
\hline $\mathrm{C}$ & -10.28405200 & -2.99838100 & 1.64482400 \\
\hline $\mathrm{C}$ & -9.06487500 & -2.41721800 & 1.25500200 \\
\hline $\mathrm{C}$ & -8.97136300 & 2.71340900 & -1.19551200 \\
\hline $\mathrm{C}$ & -10.15464600 & 3.30274500 & -1.64019900 \\
\hline $\mathrm{C}$ & -11.36171000 & 2.65284000 & -1.32884900 \\
\hline $\mathrm{C}$ & -11.40164000 & 1.41479800 & -0.67788900 \\
\hline $\mathrm{C}$ & -12.64704700 & 0.76094100 & -0.38252600 \\
\hline $\mathrm{C}$ & -12.67138700 & -0.44035900 & 0.25423700 \\
\hline $\mathrm{C}$ & 6.49798600 & -2.75929000 & -0.26852300 \\
\hline $\mathrm{C}$ & 10.36087000 & -4.35854900 & 2.38557900 \\
\hline $\mathrm{C}$ & 11.01737300 & -5.43186200 & 1.48299500 \\
\hline $\mathrm{C}$ & 8.97090800 & -4.86615200 & 2.81284700 \\
\hline $\mathrm{C}$ & 11.21358600 & -4.16857200 & 3.66344200 \\
\hline $\mathrm{C}$ & 10.20578700 & 4.62398500 & -2.40151100 \\
\hline $\mathrm{C}$ & 8.79592700 & 5.15316500 & -2.72206000 \\
\hline
\end{tabular}




\begin{tabular}{|c|c|c|c|}
\hline $\mathrm{C}$ & 10.95543100 & 4.41237000 & -3.73942700 \\
\hline $\mathrm{C}$ & 10.94801900 & 5.68864800 & -1.55716600 \\
\hline $\mathrm{C}$ & 6.50091300 & 3.02536900 & 0.40812200 \\
\hline $\mathrm{C}$ & -6.49328200 & -2.75297200 & -0.24457300 \\
\hline $\mathrm{C}$ & -6.48340100 & 3.03646000 & 0.38627900 \\
\hline $\mathrm{C}$ & -10.35626600 & -4.31397000 & 2.44113000 \\
\hline $\mathrm{C}$ & -11.04919300 & -5.39036900 & 1.57014800 \\
\hline $\mathrm{C}$ & -11.17778400 & -4.09091500 & 3.73417500 \\
\hline $\mathrm{C}$ & -8.96540000 & -4.83777700 & 2.84503800 \\
\hline $\mathrm{C}$ & -10.18647100 & 4.61939800 & -2.43581300 \\
\hline $\mathrm{C}$ & -8.77579500 & 5.14168100 & -2.76407400 \\
\hline $\mathrm{C}$ & -10.92386700 & 5.69369500 & -1.59946700 \\
\hline $\mathrm{C}$ & -10.93958800 & 4.39797500 & -3.77018400 \\
\hline $\mathrm{C}$ & 5.74017600 & -3.64246700 & 0.52303700 \\
\hline $\mathrm{C}$ & 5.71324000 & -5.00624700 & 0.24469500 \\
\hline $\mathrm{C}$ & 6.42107800 & -5.55665700 & -0.83955800 \\
\hline $\mathrm{C}$ & 7.16986000 & -4.66575500 & -1.62488300 \\
\hline $\mathrm{C}$ & 7.21569300 & -3.30119900 & -1.34496400 \\
\hline $\mathrm{C}$ & 5.71699700 & 3.93319200 & -0.32335000 \\
\hline $\mathrm{C}$ & 5.71826400 & 5.29466800 & -0.01751800 \\
\hline $\mathrm{C}$ & 6.48329500 & 5.81149200 & 1.03934400 \\
\hline $\mathrm{C}$ & 7.26250100 & 4.89236300 & 1.76908800 \\
\hline $\mathrm{C}$ & 7.28367600 & 3.53895200 & 1.46035700 \\
\hline $\mathrm{C}$ & -7.26597200 & 3.56013000 & 1.43368700 \\
\hline $\mathrm{C}$ & -7.24234800 & 4.91595300 & 1.73138200 \\
\hline $\mathrm{C}$ & -6.46086300 & 5.82759200 & 0.99468400 \\
\hline $\mathrm{C}$ & -5.69588700 & 5.30064700 & -0.05721100 \\
\hline $\mathrm{C}$ & -5.69694400 & 3.93670100 & -0.35181800 \\
\hline $\mathrm{C}$ & -7.22587500 & -3.30404600 & -1.31129200 \\
\hline $\mathrm{C}$ & -7.19180100 & -4.66854400 & -1.56864700 \\
\hline $\mathrm{C}$ & -6.44465200 & -5.55929300 & -0.77325300 \\
\hline $\mathrm{C}$ & -5.72272900 & -5.00266500 & 0.29256800 \\
\hline $\mathrm{C}$ & -5.73694900 & -3.62977500 & 0.54915300 \\
\hline $\mathrm{C}$ & 6.34939000 & -7.06662700 & -1.12332200 \\
\hline $\mathrm{C}$ & 7.19675300 & -7.47384300 & -2.34350000 \\
\hline $\mathrm{C}$ & 4.87814000 & -7.46177600 & -1.40024900 \\
\hline $\mathrm{C}$ & 6.86564100 & -7.84774300 & 0.10921100 \\
\hline $\mathrm{C}$ & 6.49608400 & 7.30191600 & 1.41475400 \\
\hline $\mathrm{C}$ & 5.59683900 & 8.14595600 & 0.49200400 \\
\hline $\mathrm{C}$ & 7.94234600 & 7.84481700 & 1.31314800 \\
\hline $\mathrm{C}$ & 5.98933000 & 7.46286200 & 2.86913100 \\
\hline $\mathrm{C}$ & -6.44364500 & -7.06331600 & -1.09300400 \\
\hline $\mathrm{C}$ & -5.58946900 & -7.87242300 & -0.09891800 \\
\hline $\mathrm{C}$ & -5.87240200 & -7.28018300 & -2.51557800 \\
\hline
\end{tabular}




\begin{tabular}{|c|c|c|c|}
\hline $\mathrm{C}$ & -7.89379500 & -7.60203200 & -1.03671700 \\
\hline $\mathrm{C}$ & -6.47123400 & 7.32108400 & 1.35774800 \\
\hline $\mathrm{C}$ & -7.91647100 & 7.86570000 & 1.25081100 \\
\hline $\mathrm{C}$ & -5.56996600 & 8.15588100 & 0.42858900 \\
\hline $\mathrm{C}$ & -5.96507000 & 7.49316500 & 2.81106100 \\
\hline $\mathrm{C}$ & 1.43750600 & 1.25394900 & 0.37507300 \\
\hline $\mathrm{C}$ & 0.71937400 & 2.39471800 & 0.75098200 \\
\hline $\mathrm{C}$ & -0.70546400 & 2.39563900 & 0.74984700 \\
\hline $\mathrm{C}$ & -1.42456200 & 1.25571000 & 0.37316200 \\
\hline $\mathrm{H}$ & 12.43520100 & -2.82699500 & 1.47451900 \\
\hline $\mathrm{H}$ & 8.15476300 & -2.93779300 & 1.50187900 \\
\hline $\mathrm{H}$ & 12.32340900 & 3.09559600 & -1.60147000 \\
\hline $\mathrm{H}$ & 8.05255300 & 3.19750500 & -1.39861500 \\
\hline $\mathrm{H}$ & 13.62721900 & -0.95175900 & 0.46687000 \\
\hline $\mathrm{H}$ & 13.58481900 & 1.23544000 & -0.67148500 \\
\hline $\mathrm{H}$ & 4.00918300 & 2.53020100 & 0.74072800 \\
\hline $\mathrm{H}$ & 4.08731800 & -2.26150100 & -0.64195900 \\
\hline $\mathrm{H}$ & -1.24129600 & -5.39448700 & -1.83641300 \\
\hline $\mathrm{H}$ & 1.25041500 & -5.39724500 & -1.82973700 \\
\hline $\mathrm{H}$ & -2.47578100 & -3.38861500 & -1.17235100 \\
\hline $\mathrm{H}$ & -3.99417200 & 2.53749900 & 0.73035200 \\
\hline $\mathrm{H}$ & -4.07910000 & -2.26018800 & -0.63217100 \\
\hline $\mathrm{H}$ & -12.42737800 & -2.78648100 & 1.51924400 \\
\hline $\mathrm{H}$ & -8.14729500 & -2.91397100 & 1.52862500 \\
\hline $\mathrm{H}$ & -8.03499100 & 3.19614700 & -1.42426000 \\
\hline $\mathrm{H}$ & -12.30666500 & 3.10423800 & -1.61734100 \\
\hline $\mathrm{H}$ & -13.57127400 & 1.25644400 & -0.66622000 \\
\hline $\mathrm{H}$ & -13.61668900 & -0.91905200 & 0.49424300 \\
\hline $\mathrm{H}$ & 10.42773500 & -5.59444400 & 0.57335300 \\
\hline $\mathrm{H}$ & 12.03040400 & -5.14742900 & 1.17976900 \\
\hline $\mathrm{H}$ & 11.08667900 & -6.38485600 & 2.01997700 \\
\hline $\mathrm{H}$ & 8.32719000 & -5.08320400 & 1.95353200 \\
\hline $\mathrm{H}$ & 8.45472500 & -4.14613100 & 3.45883300 \\
\hline $\mathrm{H}$ & 9.08237300 & -5.79510900 & 3.38186900 \\
\hline $\mathrm{H}$ & 12.24030300 & -3.86470100 & 3.43663700 \\
\hline $\mathrm{H}$ & 10.77559900 & -3.40955800 & 4.32135800 \\
\hline $\mathrm{H}$ & 11.26593900 & -5.11033000 & 4.22107700 \\
\hline $\mathrm{H}$ & 8.22143300 & 5.37317300 & -1.81570600 \\
\hline $\mathrm{H}$ & 8.87802200 & 6.08347600 & -3.29377200 \\
\hline $\mathrm{H}$ & 8.22282900 & 4.44429900 & -3.33141200 \\
\hline $\mathrm{H}$ & 10.45388000 & 3.66047900 & -4.35901900 \\
\hline $\mathrm{H}$ & 10.98451600 & 5.35113500 & -4.30361500 \\
\hline $\mathrm{H}$ & 11.99041600 & 4.08861200 & -3.58954400 \\
\hline $\mathrm{H}$ & 11.97578600 & 5.38845000 & -1.32708200 \\
\hline
\end{tabular}




\begin{tabular}{|c|c|c|c|}
\hline $\mathrm{H}$ & 10.99642500 & 6.63606300 & -2.10590600 \\
\hline $\mathrm{H}$ & 10.43018000 & 5.87005600 & -0.60846100 \\
\hline $\mathrm{H}$ & -10.48220200 & -5.57802300 & 0.65112900 \\
\hline $\mathrm{H}$ & -11.12212800 & -6.33315400 & 2.12437700 \\
\hline $\mathrm{H}$ & -12.06412100 & -5.09522700 & 1.28420900 \\
\hline $\mathrm{H}$ & -10.71282100 & -3.33019700 & 4.37130600 \\
\hline $\mathrm{H}$ & -12.20344700 & -3.77183500 & 3.52385900 \\
\hline $\mathrm{H}$ & -11.23462000 & -5.02343800 & 4.30675800 \\
\hline $\mathrm{H}$ & -8.42588300 & -4.12042000 & 3.47467200 \\
\hline $\mathrm{H}$ & -9.07874300 & -5.76026800 & 3.42419100 \\
\hline $\mathrm{H}$ & -8.34228400 & -5.07020100 & 1.97451900 \\
\hline $\mathrm{H}$ & -8.20596600 & 4.42571200 & -3.36816300 \\
\hline $\mathrm{H}$ & -8.85648600 & 6.06700200 & -3.34402000 \\
\hline $\mathrm{H}$ & -8.19877400 & 5.36830100 & -1.86095000 \\
\hline $\mathrm{H}$ & -11.95203600 & 5.39857900 & -1.36467200 \\
\hline $\mathrm{H}$ & -10.40357700 & 5.88196700 & -0.65344400 \\
\hline $\mathrm{H}$ & -10.97061200 & 6.63634700 & -2.15649300 \\
\hline $\mathrm{H}$ & -10.44144100 & 3.63923800 & -4.38415300 \\
\hline $\mathrm{H}$ & -10.96733700 & 5.33176900 & -4.34263100 \\
\hline $\mathrm{H}$ & -11.97512200 & 4.07839900 & -3.61518900 \\
\hline $\mathrm{H}$ & 5.19419600 & -3.26150000 & 1.38230900 \\
\hline $\mathrm{H}$ & 5.13033400 & -5.65336700 & 0.89347200 \\
\hline $\mathrm{H}$ & 7.72996000 & -5.03167600 & -2.47759000 \\
\hline $\mathrm{H}$ & 7.80643100 & -2.64628400 & -1.97867900 \\
\hline $\mathrm{H}$ & 5.12893500 & 3.57848800 & -1.16567200 \\
\hline $\mathrm{H}$ & 5.11244300 & 5.95707200 & -0.62515000 \\
\hline $\mathrm{H}$ & 7.86698100 & 5.24024400 & 2.60111300 \\
\hline $\mathrm{H}$ & 7.89626200 & 2.86429800 & 2.05095900 \\
\hline $\mathrm{H}$ & -7.88035300 & 2.89152600 & 2.02927900 \\
\hline $\mathrm{H}$ & -7.84673300 & 5.27179400 & 2.56009500 \\
\hline $\mathrm{H}$ & -5.08822300 & 5.95686100 & -0.66969600 \\
\hline $\mathrm{H}$ & -5.10869300 & 3.57390500 & -1.19055200 \\
\hline $\mathrm{H}$ & -7.81626100 & -2.65230300 & -1.94850300 \\
\hline $\mathrm{H}$ & -7.76101400 & -5.04751100 & -2.41202000 \\
\hline $\mathrm{H}$ & -5.14014700 & -5.64001300 & 0.94802200 \\
\hline $\mathrm{H}$ & -5.18100100 & -3.24245700 & 1.39915600 \\
\hline $\mathrm{H}$ & 6.85858400 & -6.98123600 & -3.26224500 \\
\hline $\mathrm{H}$ & 7.11518500 & -8.55401800 & -2.50241800 \\
\hline $\mathrm{H}$ & 8.25841700 & -7.24387800 & -2.19972400 \\
\hline $\mathrm{H}$ & 4.48692200 & -6.93064100 & -2.27610100 \\
\hline $\mathrm{H}$ & 4.80800000 & -8.53708900 & -1.59907600 \\
\hline $\mathrm{H}$ & 4.22715800 & -7.23970900 & -0.54744200 \\
\hline $\mathrm{H}$ & 7.90892500 & -7.59373500 & 0.32758600 \\
\hline $\mathrm{H}$ & 6.27362400 & -7.64043900 & 1.00687500 \\
\hline
\end{tabular}




$\begin{array}{lrrr}\mathrm{H} & 6.81365900 & -8.92578600 & -0.08014900 \\ \mathrm{H} & 5.92021500 & 8.09658600 & -0.55404000 \\ \mathrm{H} & 4.54721300 & 7.83415000 & 0.54435000 \\ \mathrm{H} & 5.64105800 & 9.19604600 & 0.79844800 \\ \mathrm{H} & 8.32841700 & 7.75096000 & 0.29171700 \\ \mathrm{H} & 8.62837100 & 7.31699000 & 1.98369700 \\ \mathrm{H} & 7.96494200 & 8.90573800 & 1.58616800 \\ \mathrm{H} & 4.96269400 & 7.09332900 & 2.97280800 \\ \mathrm{H} & 6.61593400 & 6.92138000 & 3.58532900 \\ \mathrm{H} & 5.99857800 & 8.52078500 & 3.15434500 \\ \mathrm{H} & -4.53731800 & -7.56459400 & -0.11545000 \\ \mathrm{H} & -5.62336500 & -8.93373900 & -0.36556100 \\ \mathrm{H} & -5.95891500 & -7.78108500 & 0.92876500 \\ \mathrm{H} & -4.84074700 & -6.91592500 & -2.58657100 \\ \mathrm{H} & -6.46381100 & -6.76462100 & -3.27924600 \\ \mathrm{H} & -5.87141900 & -8.34789900 & -2.76193300 \\ \mathrm{H} & -8.54750600 & -7.10026200 & -1.75760900 \\ \mathrm{H} & -7.90635900 & -8.67278300 & -1.26918600 \\ \mathrm{H} & -8.32583100 & -7.46812400 & -0.03855900 \\ \mathrm{H} & -8.60379600 & 7.34480900 & 1.92544000 \\ \mathrm{H} & -7.93727300 & 8.92892500 & 1.51486700 \\ \mathrm{H} & -8.30220100 & 7.76392700 & 0.23001000 \\ \mathrm{H} & -5.89275700 & 8.09833300 & -0.61721800 \\ \mathrm{H} & -5.61259900 & 9.20856200 & 0.72624100 \\ \mathrm{H} & -4.52090300 & 7.84276000 & 0.48421700 \\ \mathrm{H} & -4.93910300 & 7.12281800 & 2.91839600 \\ \mathrm{H} & -5.97275100 & 8.55341700 & 3.08753600 \\ \mathrm{H} & -6.59299000 & 6.95863100 & 3.53131700 \\ \mathrm{H} & 1.23123700 & 3.30274400 & 1.05673000 \\ \mathrm{H} & -1.21662600 & 3.30435400 & 1.05472600 \\ \mathrm{H} & 2.48515800 & -3.39349300 & -1.16251400\end{array}$

\section{OT $9 a^{2 \cdot+}$}

Energy (with Zero Point Energy correction $)=-4562.573678$ (-4560.635667) Hartree Free energy $(298 \mathrm{~K})=-4560.785176$ Hartree

Zero number of imaginary frequency

23

$\begin{array}{lrrr}\mathrm{C} & 11.49273700 & -2.38788600 & 1.20954900 \\ \mathrm{C} & 11.47518100 & -1.13633500 & 0.57023300 \\ \mathrm{C} & 10.22004800 & -0.52306200 & 0.26249900 \\ \mathrm{C} & 9.01259300 & -1.22610700 & 0.51612200 \\ \mathrm{C} & 9.09424700 & -2.44725500 & 1.19754100 \\ \mathrm{C} & 10.31800300 & -3.04233600 & 1.57044500 \\ \mathrm{C} & 10.19462500 & 0.77958100 & -0.30785800\end{array}$




\begin{tabular}{|c|c|c|c|}
\hline $\mathrm{C}$ & 8.96112000 & 1.47865100 & -0.47686900 \\
\hline $\mathrm{C}$ & 7.72770500 & 0.84682800 & -0.04009800 \\
\hline $\mathrm{C}$ & 7.73338500 & -0.58595200 & 0.15195300 \\
\hline $\mathrm{C}$ & 11.41299600 & 1.41444600 & -0.67594300 \\
\hline $\mathrm{C}$ & 11.37235600 & 2.66833600 & -1.30873900 \\
\hline $\mathrm{C}$ & 10.16379900 & 3.32141800 & -1.61107200 \\
\hline $\mathrm{C}$ & 8.98588800 & 2.72075700 & -1.17371700 \\
\hline $\mathrm{C}$ & 12.68345100 & -0.45784800 & 0.21967300 \\
\hline $\mathrm{C}$ & 12.65617000 & 0.76163500 & -0.39708000 \\
\hline $\mathrm{C}$ & 6.50302200 & 1.57285900 & 0.13847100 \\
\hline $\mathrm{C}$ & 5.27762900 & 0.85934300 & 0.15388900 \\
\hline $\mathrm{C}$ & 5.28928700 & -0.55794600 & -0.09991900 \\
\hline $\mathrm{C}$ & 6.52583200 & -1.28410700 & -0.03587000 \\
\hline $\mathrm{C}$ & 4.01773300 & 1.50393200 & 0.38854100 \\
\hline $\mathrm{C}$ & 2.84004700 & 0.82919500 & 0.20892500 \\
\hline $\mathrm{C}$ & 2.84825600 & -0.56446400 & -0.21970400 \\
\hline $\mathrm{C}$ & 4.05712200 & -1.22164000 & -0.35037400 \\
\hline $\mathrm{C}$ & -0.70254200 & -4.51286200 & -1.50940600 \\
\hline $\mathrm{C}$ & 0.70960600 & -4.51424700 & -1.50696700 \\
\hline $\mathrm{C}$ & 1.40483600 & -3.38312600 & -1.14041400 \\
\hline $\mathrm{C}$ & 0.74166100 & -2.18874900 & -0.75422800 \\
\hline $\mathrm{C}$ & -0.73335000 & -2.18800600 & -0.75477000 \\
\hline $\mathrm{C}$ & -1.39724800 & -3.38105000 & -1.14385200 \\
\hline $\mathrm{C}$ & 1.45343800 & -1.00665600 & -0.37560800 \\
\hline $\mathrm{C}$ & 0.69225100 & 0.11378200 & -0.02346900 \\
\hline $\mathrm{C}$ & -0.68196600 & 0.11431100 & -0.02352400 \\
\hline $\mathrm{C}$ & -1.44426000 & -1.00563600 & -0.37515200 \\
\hline $\mathrm{C}$ & -2.83873400 & -0.56249400 & -0.21791600 \\
\hline $\mathrm{C}$ & -2.82888700 & 0.83211100 & 0.20752900 \\
\hline $\mathrm{C}$ & -4.00574100 & 1.50915800 & 0.38405100 \\
\hline $\mathrm{C}$ & -5.26609600 & 0.86526700 & 0.15110100 \\
\hline $\mathrm{C}$ & -5.27969200 & -0.55350500 & -0.09404300 \\
\hline $\mathrm{C}$ & -4.04849100 & -1.21961900 & -0.34308400 \\
\hline $\mathrm{C}$ & -6.49027200 & 1.58060100 & 0.12987600 \\
\hline $\mathrm{C}$ & -7.71594100 & 0.85536900 & -0.04469400 \\
\hline $\mathrm{C}$ & -7.72431700 & -0.57568100 & 0.15866600 \\
\hline $\mathrm{C}$ & -6.51744300 & -1.27726400 & -0.02327800 \\
\hline $\mathrm{C}$ & -8.94778500 & 1.48674100 & -0.48626000 \\
\hline $\mathrm{C}$ & -10.18289400 & 0.79240200 & -0.31016100 \\
\hline $\mathrm{C}$ & -10.21092300 & -0.50519600 & 0.27144400 \\
\hline $\mathrm{C}$ & -9.00496000 & -1.20994200 & 0.52893400 \\
\hline $\mathrm{C}$ & -11.46753200 & -1.11149000 & 0.58708400 \\
\hline $\mathrm{C}$ & -11.48793400 & -2.35664400 & 1.23815700 \\
\hline $\mathrm{C}$ & -10.31459000 & -3.01251800 & 1.60134700 \\
\hline
\end{tabular}




\begin{tabular}{|c|c|c|c|}
\hline $\mathrm{C}$ & -9.08956500 & -2.42566500 & 1.22001900 \\
\hline $\mathrm{C}$ & -8.96955800 & 2.72294600 & -1.19328400 \\
\hline $\mathrm{C}$ & -10.14622000 & 3.32296400 & -1.63481300 \\
\hline $\mathrm{C}$ & -11.35633000 & 2.67579400 & -1.32580500 \\
\hline $\mathrm{C}$ & -11.39991700 & 1.42767300 & -0.68220100 \\
\hline $\mathrm{C}$ & -12.64469100 & 0.78104700 & -0.39557000 \\
\hline $\mathrm{C}$ & -12.67448800 & -0.43239700 & 0.23246900 \\
\hline $\mathrm{C}$ & 6.49411600 & -2.75996700 & -0.26342600 \\
\hline $\mathrm{C}$ & 10.38553000 & -4.38232500 & 2.32659700 \\
\hline $\mathrm{C}$ & 10.99506000 & -5.45069500 & 1.38602400 \\
\hline $\mathrm{C}$ & 8.99897400 & -4.87026400 & 2.78720900 \\
\hline $\mathrm{C}$ & 11.27853500 & -4.23079200 & 3.58151600 \\
\hline $\mathrm{C}$ & 10.19264600 & 4.64557900 & -2.39390900 \\
\hline $\mathrm{C}$ & 8.78007700 & 5.16651100 & -2.71509900 \\
\hline $\mathrm{C}$ & 10.94510600 & 4.43765700 & -3.73089800 \\
\hline $\mathrm{C}$ & 10.92907100 & 5.71231600 & -1.54728600 \\
\hline $\mathrm{C}$ & 6.51759300 & 3.03124000 & 0.40951900 \\
\hline $\mathrm{C}$ & -6.48885900 & -2.75516500 & -0.23527100 \\
\hline $\mathrm{C}$ & -6.50269500 & 3.04076800 & 0.39091500 \\
\hline $\mathrm{C}$ & -10.38728000 & -4.34165800 & 2.37595700 \\
\hline $\mathrm{C}$ & -11.04783300 & -5.41051600 & 1.47128400 \\
\hline $\mathrm{C}$ & -11.23882700 & -4.15099900 & 3.65442100 \\
\hline $\mathrm{C}$ & -8.99857400 & -4.85422900 & 2.80142200 \\
\hline $\mathrm{C}$ & -10.17211600 & 4.64063500 & -2.42859700 \\
\hline $\mathrm{C}$ & -8.75840300 & 5.15493900 & -2.75547400 \\
\hline $\mathrm{C}$ & -10.90468100 & 5.71644400 & -1.59013300 \\
\hline $\mathrm{C}$ & -10.92649200 & 4.42382100 & -3.76308900 \\
\hline $\mathrm{C}$ & 5.77471500 & -3.63382200 & 0.57062600 \\
\hline $\mathrm{C}$ & 5.73116900 & -5.00019300 & 0.30508500 \\
\hline $\mathrm{C}$ & 6.38909600 & -5.56223000 & -0.80437000 \\
\hline $\mathrm{C}$ & 7.10193700 & -4.68047900 & -1.63052500 \\
\hline $\mathrm{C}$ & 7.15739600 & -3.31150400 & -1.36750400 \\
\hline $\mathrm{C}$ & 5.72791400 & 3.94658600 & -0.30883200 \\
\hline $\mathrm{C}$ & 5.73875600 & 5.30556400 & 0.00243900 \\
\hline $\mathrm{C}$ & 6.51812400 & 5.81608100 & 1.05387000 \\
\hline $\mathrm{C}$ & 7.30424600 & 4.89217000 & 1.76920300 \\
\hline $\mathrm{C}$ & 7.31687800 & 3.54015200 & 1.45382700 \\
\hline $\mathrm{C}$ & -7.30269800 & 3.55837800 & 1.43039800 \\
\hline $\mathrm{C}$ & -7.28821900 & 4.91254100 & 1.73625000 \\
\hline $\mathrm{C}$ & -6.49955500 & 5.83008500 & 1.01551500 \\
\hline $\mathrm{C}$ & -5.71939100 & 5.31084600 & -0.03103900 \\
\hline $\mathrm{C}$ & -5.71026200 & 3.94966900 & -0.33258900 \\
\hline $\mathrm{C}$ & -7.16236500 & -3.31835500 & -1.33210200 \\
\hline $\mathrm{C}$ & -7.11494400 & -4.68708700 & -1.57202700 \\
\hline
\end{tabular}




\begin{tabular}{|c|c|c|c|}
\hline $\mathrm{C}$ & -6.40476700 & -5.56599800 & -0.73278900 \\
\hline $\mathrm{C}$ & -5.73775400 & -4.99541800 & 0.36106000 \\
\hline $\mathrm{C}$ & -5.77196000 & -3.61998500 & 0.60410300 \\
\hline $\mathrm{C}$ & 6.29921300 & -7.07464100 & -1.07231200 \\
\hline $\mathrm{C}$ & 7.09674000 & -7.49617800 & -2.32089700 \\
\hline $\mathrm{C}$ & 4.81665500 & -7.46449300 & -1.28918400 \\
\hline $\mathrm{C}$ & 6.85880200 & -7.84941700 & 0.14486700 \\
\hline $\mathrm{C}$ & 6.53513700 & 7.30391200 & 1.43804700 \\
\hline $\mathrm{C}$ & 5.62788400 & 8.15441600 & 0.52911300 \\
\hline $\mathrm{C}$ & 7.98070300 & 7.84710500 & 1.32735000 \\
\hline $\mathrm{C}$ & 6.04152500 & 7.45498800 & 2.89820400 \\
\hline $\mathrm{C}$ & -6.37773900 & -7.07323100 & -1.03935300 \\
\hline $\mathrm{C}$ & -5.57467300 & -7.86879300 & 0.00697500 \\
\hline $\mathrm{C}$ & -5.72726700 & -7.29806200 & -2.42602200 \\
\hline $\mathrm{C}$ & -7.82482600 & -7.62180700 & -1.05856800 \\
\hline $\mathrm{C}$ & -6.51479800 & 7.32063800 & 1.38903600 \\
\hline $\mathrm{C}$ & -7.95938200 & 7.86524900 & 1.27259100 \\
\hline $\mathrm{C}$ & -5.60506800 & 8.16325800 & 0.47525000 \\
\hline $\mathrm{C}$ & -6.02286300 & 7.48139100 & 2.84873100 \\
\hline $\mathrm{C}$ & 1.43772100 & 1.25660000 & 0.34648600 \\
\hline $\mathrm{C}$ & 0.72402900 & 2.39284700 & 0.71114900 \\
\hline $\mathrm{C}$ & -0.71163000 & 2.39366900 & 0.71039000 \\
\hline $\mathrm{C}$ & -1.42630400 & 1.25810700 & 0.34543000 \\
\hline $\mathrm{H}$ & 12.45841500 & -2.82965500 & 1.43606800 \\
\hline $\mathrm{H}$ & 8.17848700 & -2.94925500 & 1.46729500 \\
\hline $\mathrm{H}$ & 12.31711500 & 3.12242100 & -1.59352100 \\
\hline $\mathrm{H}$ & 8.04587800 & 3.19545400 & -1.40422900 \\
\hline $\mathrm{H}$ & 13.63195500 & -0.93729800 & 0.44508300 \\
\hline $\mathrm{H}$ & 13.58067000 & 1.26140400 & -0.67201600 \\
\hline $\mathrm{H}$ & 4.00634000 & 2.53930800 & 0.70554200 \\
\hline $\mathrm{H}$ & 4.08988200 & -2.26547800 & -0.62665400 \\
\hline $\mathrm{H}$ & -1.24262200 & -5.40769300 & -1.80371300 \\
\hline $\mathrm{H}$ & 1.24954400 & -5.41026900 & -1.79794900 \\
\hline $\mathrm{H}$ & -2.48041000 & -3.39989800 & -1.15777200 \\
\hline $\mathrm{H}$ & -3.99323400 & 2.54571600 & 0.69717400 \\
\hline $\mathrm{H}$ & -4.08268600 & -2.26512000 & -0.61311800 \\
\hline $\mathrm{H}$ & -12.45457900 & -2.79241700 & 1.47234900 \\
\hline $\mathrm{H}$ & -8.17509500 & -2.92912600 & 1.49128400 \\
\hline $\mathrm{H}$ & -8.02839900 & 3.19322300 & -1.42822600 \\
\hline $\mathrm{H}$ & -12.30008400 & 3.13018600 & -1.61342100 \\
\hline $\mathrm{H}$ & -13.56812000 & 1.28106400 & -0.67363900 \\
\hline $\mathrm{H}$ & -13.62396400 & -0.90696400 & 0.46404100 \\
\hline $\mathrm{H}$ & 10.37641600 & -5.58592800 & 0.49137700 \\
\hline $\mathrm{H}$ & 12.00397500 & -5.17763900 & 1.05881500 \\
\hline
\end{tabular}




\begin{tabular}{|c|c|c|c|}
\hline $\mathrm{H}$ & 11.06231200 & -6.41451200 & 1.90352700 \\
\hline $\mathrm{H}$ & 8.32856000 & -5.07181900 & 1.94506100 \\
\hline $\mathrm{H}$ & 8.51228500 & -4.14650800 & 3.45174300 \\
\hline $\mathrm{H}$ & 9.11094100 & -5.80482000 & 3.34678200 \\
\hline $\mathrm{H}$ & 12.30590200 & -3.94846800 & 3.33088400 \\
\hline $\mathrm{H}$ & 10.87735000 & -3.47257200 & 4.26338800 \\
\hline $\mathrm{H}$ & 11.32436800 & -5.18202600 & 4.12312500 \\
\hline $\mathrm{H}$ & 8.20309600 & 5.38117300 & -1.80910100 \\
\hline $\mathrm{H}$ & 8.85741100 & 6.09838200 & -3.28472900 \\
\hline $\mathrm{H}$ & 8.21217400 & 4.45577000 & -3.32709700 \\
\hline $\mathrm{H}$ & 10.44849700 & 3.68342000 & -4.35152100 \\
\hline $\mathrm{H}$ & 10.96953300 & 5.37671700 & -4.29454800 \\
\hline $\mathrm{H}$ & 11.98217300 & 4.12042800 & -3.58039900 \\
\hline $\mathrm{H}$ & 11.95815100 & 5.41693700 & -1.31606500 \\
\hline $\mathrm{H}$ & 10.97447400 & 6.65999600 & -2.09556100 \\
\hline $\mathrm{H}$ & 10.40908100 & 5.89134400 & -0.59938800 \\
\hline $\mathrm{H}$ & -10.45922600 & -5.57483400 & 0.56150200 \\
\hline $\mathrm{H}$ & -11.12077500 & -6.36396000 & 2.00686700 \\
\hline $\mathrm{H}$ & -12.06007500 & -5.12150400 & 1.16936900 \\
\hline $\mathrm{H}$ & -10.79884500 & -3.39411300 & 4.31349100 \\
\hline $\mathrm{H}$ & -12.26519200 & -3.84485800 & 3.42857400 \\
\hline $\mathrm{H}$ & -11.29303300 & -5.09338600 & 4.21064000 \\
\hline $\mathrm{H}$ & -8.47925000 & -4.13739200 & 3.44846100 \\
\hline $\mathrm{H}$ & -9.11358000 & -5.78336200 & 3.36944000 \\
\hline $\mathrm{H}$ & -8.35584800 & -5.07220200 & 1.94182100 \\
\hline $\mathrm{H}$ & -8.19303900 & 4.43747500 & -3.36195100 \\
\hline $\mathrm{H}$ & -8.83369700 & 6.08217900 & -3.33288200 \\
\hline $\mathrm{H}$ & -8.17997700 & 5.37567500 & -1.85185000 \\
\hline $\mathrm{H}$ & -11.93441000 & 5.42597600 & -1.35562400 \\
\hline $\mathrm{H}$ & -10.38334400 & 5.90166800 & -0.64416500 \\
\hline $\mathrm{H}$ & -10.94778600 & 6.65973900 & -2.14610700 \\
\hline $\mathrm{H}$ & -10.43255900 & 3.66317000 & -4.37799500 \\
\hline $\mathrm{H}$ & -10.94894700 & 5.35829200 & -4.33440300 \\
\hline $\mathrm{H}$ & -11.96425100 & 4.11063600 & -3.60894100 \\
\hline $\mathrm{H}$ & 5.25981000 & -3.24256200 & 1.44447500 \\
\hline $\mathrm{H}$ & 5.17202500 & -5.63923300 & 0.98232300 \\
\hline $\mathrm{H}$ & 7.62350400 & -5.05482300 & -2.50387700 \\
\hline $\mathrm{H}$ & 7.71660900 & -2.66253600 & -2.03555400 \\
\hline $\mathrm{H}$ & 5.12686600 & 3.59774800 & -1.14402100 \\
\hline $\mathrm{H}$ & 5.12826900 & 5.97314200 & -0.59470100 \\
\hline $\mathrm{H}$ & 7.91910900 & 5.23435700 & 2.59592900 \\
\hline $\mathrm{H}$ & 7.93013600 & 2.85980700 & 2.03703800 \\
\hline $\mathrm{H}$ & -7.91801800 & 2.88320700 & 2.01742900 \\
\hline $\mathrm{H}$ & -7.90369300 & 5.26158200 & 2.55964300 \\
\hline
\end{tabular}




\begin{tabular}{|c|c|c|c|}
\hline $\mathrm{H}$ & -5.10687500 & 5.97315600 & -0.63195500 \\
\hline $\mathrm{H}$ & -5.10845200 & 3.59389900 & -1.16429400 \\
\hline $\mathrm{H}$ & -7.72044800 & -2.67459000 & -2.00603300 \\
\hline $\mathrm{H}$ & -7.64134100 & -5.07652900 & -2.43833300 \\
\hline $\mathrm{H}$ & -5.18040900 & -5.62273900 & 1.04748400 \\
\hline $\mathrm{H}$ & -5.25031100 & -3.22040700 & 1.47017100 \\
\hline $\mathrm{H}$ & 6.72437400 & -7.01073400 & -3.23016600 \\
\hline $\mathrm{H}$ & 7.00564000 & -8.57753800 & -2.46663000 \\
\hline $\mathrm{H}$ & 8.16394900 & -7.26818600 & -2.22108500 \\
\hline $\mathrm{H}$ & 4.39482800 & -6.93790900 & -2.15347800 \\
\hline $\mathrm{H}$ & 4.73287200 & -8.54104000 & -1.47626000 \\
\hline $\mathrm{H}$ & 4.20009300 & -7.23114900 & -0.41423200 \\
\hline $\mathrm{H}$ & 7.91155500 & -7.60034200 & 0.31975900 \\
\hline $\mathrm{H}$ & 6.30402700 & -7.63093900 & 1.06342100 \\
\hline $\mathrm{H}$ & 6.79291100 & -8.92876700 & -0.03279400 \\
\hline $\mathrm{H}$ & 5.94121500 & 8.11159200 & -0.52029400 \\
\hline $\mathrm{H}$ & 4.57858300 & 7.84327000 & 0.58955800 \\
\hline $\mathrm{H}$ & 5.67600400 & 9.20243700 & 0.84189900 \\
\hline $\mathrm{H}$ & 8.35749200 & 7.76108400 & 0.30172300 \\
\hline $\mathrm{H}$ & 8.67265800 & 7.31461500 & 1.98806500 \\
\hline $\mathrm{H}$ & 8.00567800 & 8.90597800 & 1.60786500 \\
\hline $\mathrm{H}$ & 5.01611500 & 7.08434600 & 3.00879200 \\
\hline $\mathrm{H}$ & 6.67490900 & 6.90916000 & 3.60504400 \\
\hline $\mathrm{H}$ & 6.05305700 & 8.51112800 & 3.18979200 \\
\hline $\mathrm{H}$ & -4.52481200 & -7.55542200 & 0.04448400 \\
\hline $\mathrm{H}$ & -5.58899900 & -8.93280700 & -0.25093200 \\
\hline $\mathrm{H}$ & -5.99943200 & -7.76949900 & 1.01234300 \\
\hline $\mathrm{H}$ & -4.69585300 & -6.92666000 & -2.44255800 \\
\hline $\mathrm{H}$ & -6.27886300 & -6.79209400 & -3.22517800 \\
\hline $\mathrm{H}$ & -5.70516500 & -8.36763600 & -2.66368400 \\
\hline $\mathrm{H}$ & -8.44131900 & -7.12974200 & -1.81797900 \\
\hline $\mathrm{H}$ & -7.81835400 & -8.69441700 & -1.28290400 \\
\hline $\mathrm{H}$ & -8.31181200 & -7.48332200 & -0.08655200 \\
\hline $\mathrm{H}$ & -8.65299100 & 7.33867700 & 1.93630500 \\
\hline $\mathrm{H}$ & -7.98303100 & 8.92617300 & 1.54536900 \\
\hline $\mathrm{H}$ & -8.33504300 & 7.77234300 & 0.24715400 \\
\hline $\mathrm{H}$ & -5.91706100 & 8.11335700 & -0.57424300 \\
\hline $\mathrm{H}$ & -5.65204700 & 9.21356800 & 0.78044300 \\
\hline $\mathrm{H}$ & -4.55631100 & 7.85101000 & 0.53933800 \\
\hline $\mathrm{H}$ & -4.99811100 & 7.11013100 & 2.96328200 \\
\hline $\mathrm{H}$ & -6.03331300 & 8.53959300 & 3.13279300 \\
\hline $\mathrm{H}$ & -6.65792100 & 6.94146700 & 3.55859900 \\
\hline $\mathrm{H}$ & 1.23276600 & 3.30551500 & 1.00885500 \\
\hline $\mathrm{H}$ & -1.21963000 & 3.30696300 & 1.00744200 \\
\hline
\end{tabular}


$\mathrm{H} \quad 2.48796300 \quad-3.40428700 \quad-1.15026500$

Mulliken spin densities:

$\begin{array}{lll}1 & \mathrm{C} & -0.011999\end{array}$

2 C 0.036771

3 C 0.027305

$\begin{array}{lll}4 & \text { C } & -0.007204\end{array}$

$\begin{array}{lll}5 & \mathrm{C} & 0.037330\end{array}$

$\begin{array}{lll}6 & \mathrm{C} & 0.016408\end{array}$

7 C 0.011133

$\begin{array}{lll}8 & \mathrm{C} & 0.075398\end{array}$

$\begin{array}{lll}9 & \mathrm{C} & 0.069175\end{array}$

10 C -0.028083

$\begin{array}{lll}11 & \mathrm{C} & -0.055505\end{array}$

$\begin{array}{lll}12 & \mathrm{C} & 0.213719\end{array}$

13 C -0.051689

$\begin{array}{lll}14 & \mathrm{C} & 0.097511\end{array}$

$\begin{array}{lll}15 & \mathrm{C} & -0.004976\end{array}$

$\begin{array}{lll}16 & \mathrm{C} & 0.073634\end{array}$

$\begin{array}{lll}17 & \mathrm{C} & 0.142852\end{array}$

$\begin{array}{lll}18 & \mathrm{C} & -0.039934\end{array}$

19 C 0.014290

20 C 0.069706

$\begin{array}{lll}21 & \mathrm{C} & 0.080657\end{array}$

$\begin{array}{llll}22 & \mathrm{C} & -0.035061\end{array}$

$23 \mathrm{C} \quad 0.035140$

24 C 0.060125

$\begin{array}{lll}25 & \mathrm{C} & 0.010169\end{array}$

$\begin{array}{lll}26 & \mathrm{C} & 0.009812\end{array}$

27 C 0.020629

28 C -0.004017

$\begin{array}{lll}29 & \mathrm{C} & -0.003566\end{array}$

$\begin{array}{lll}30 & \mathrm{C} & 0.020257\end{array}$

31 C 0.070963

$\begin{array}{llll}32 & \mathrm{C} & -0.006298\end{array}$

33 C -0.006064

$\begin{array}{lll}34 & \mathrm{C} & 0.070129\end{array}$

35 C 0.035240

$\begin{array}{lll}36 & \mathrm{C} & -0.034903\end{array}$

$\begin{array}{lll}37 & \mathrm{C} & 0.081222\end{array}$

$\begin{array}{llll}38 & \mathrm{C} & -0.040350\end{array}$

$39 \mathrm{C} \quad 0.013745$

$\begin{array}{lll}40 & \mathrm{C} & 0.060596\end{array}$

$41 \quad \mathrm{C} \quad 0.144239$

$\begin{array}{lll}42 & \mathrm{C} & 0.068154\end{array}$ 


$\begin{array}{lcc}43 & \mathrm{C} & -0.028741 \\ 44 & \mathrm{C} & 0.071167 \\ 45 & \mathrm{C} & 0.075673 \\ 46 & \mathrm{C} & 0.011384 \\ 47 & \mathrm{C} & 0.027439 \\ 48 & \mathrm{C} & -0.006504 \\ 49 & \mathrm{C} & 0.036596 \\ 50 & \mathrm{C} & -0.012853 \\ 51 & \mathrm{C} & 0.017117 \\ 52 & \mathrm{C} & 0.035819 \\ 53 & \mathrm{C} & 0.095776 \\ 54 & \mathrm{C} & -0.050726 \\ 55 & \mathrm{C} & 0.211810 \\ 56 & \mathrm{C} & -0.055276 \\ 57 & \mathrm{C} & 0.072837 \\ 58 & \mathrm{C} & -0.005442 \\ 59 & \mathrm{C} & -0.004983 \\ 60 & \mathrm{C} & -0.001060 \\ 61 & \mathrm{C} & 0.001018 \\ 62 & \mathrm{C} & 0.000184 \\ 63 & \mathrm{C} & 0.000755 \\ 64 & \mathrm{C} & 0.002462 \\ 65 & \mathrm{C} & 0.000036 \\ 66 & \mathrm{C} & -0.000047 \\ 67 & \mathrm{C} & 0.000222 \\ 68 & \mathrm{C} & 0.004780 \\ 69 & \mathrm{C} & -0.005038 \\ 70 & \mathrm{C} & 0.004627 \\ 71 & \mathrm{C} & -0.001095 \\ 72 & \mathrm{C} & 0.001012 \\ 73 & \mathrm{C} & 0.000809 \\ 74 & \mathrm{C} & 0.000169 \\ 75 & \mathrm{C} & 0.002413 \\ 76 & \mathrm{C} & 0.000031 \\ 77 & \mathrm{C} & 0.000241 \\ 78 & \mathrm{C} & -0.000032 \\ 79 & \mathrm{C} & 0.004310 \\ 80 & \mathrm{C} & -0.001489 \\ 81 & \mathrm{C} & 0.003260 \\ 82 & \mathrm{C} & 0.000026 \\ 83 & \mathrm{C} & 0.002459 \\ 84 & \mathrm{C} & 0.021919 \\ 85 & \mathrm{C} & -0.007615 \\ 86 & \mathrm{C} & 0.041372\end{array}$




$\begin{array}{rcc}87 & \mathrm{C} & -0.016545 \\ 88 & \mathrm{C} & 0.028717 \\ 89 & \mathrm{C} & 0.028823 \\ 90 & \mathrm{C} & -0.016604 \\ 91 & \mathrm{C} & 0.041573 \\ 92 & \mathrm{C} & -0.007747 \\ 93 & \mathrm{C} & 0.022125 \\ 94 & \mathrm{C} & 0.002332 \\ 95 & \mathrm{C} & 0.000146 \\ 96 & \mathrm{C} & 0.003576 \\ 97 & \mathrm{C} & -0.001623 \\ 98 & \mathrm{C} & 0.004840 \\ 99 & \mathrm{C} & -0.000192 \\ 100 & \mathrm{C} & 0.000010 \\ 101 & \mathrm{C} & 0.000124 \\ 102 & \mathrm{C} & 0.000131 \\ 103 & \mathrm{C} & -0.002378 \\ 104 & \mathrm{C} & 0.000088 \\ 105 & \mathrm{C} & 0.001526 \\ 106 & \mathrm{C} & 0.001734 \\ 107 & \mathrm{C} & -0.000209 \\ 108 & \mathrm{C} & 0.000002 \\ 109 & \mathrm{C} & 0.000146 \\ 110 & \mathrm{C} & 0.000136 \\ 111 & \mathrm{C} & -0.002391 \\ 112 & \mathrm{C} & 0.001532 \\ 113 & \mathrm{C} & 0.000088 \\ 114 & \mathrm{C} & 0.001741 \\ 115 & \mathrm{C} & 0.018209 \\ 116 & \mathrm{C} & 0.008958 \\ 117 & \mathrm{C} & 0.009946 \\ 118 & \mathrm{C} & 0.017371 \\ 119 & \mathrm{H} & 0.000270 \\ 120 & \mathrm{H} & -0.001621 \\ 121 & \mathrm{H} & -0.009054 \\ 122 & \mathrm{H} & -0.004724 \\ 123 & \mathrm{H} & 0.000024 \\ 124 & \mathrm{H} & -0.003029 \\ 125 & \mathrm{H} & -0.002951 \\ 126 & \mathrm{H} & -0.002513 \\ 127 & \mathrm{H} & -0.000634 \\ 128 & \mathrm{H} & -0.000618 \\ 129 & \mathrm{H} & -0.000914 \\ 130 & \mathrm{H} & -0.002966\end{array}$




$\begin{array}{rlr}131 & \mathrm{H} & -0.002531 \\ 132 & \mathrm{H} & 0.000308 \\ 133 & \mathrm{H} & -0.001561 \\ 134 & \mathrm{H} & -0.004652 \\ 135 & \mathrm{H} & -0.008973 \\ 136 & \mathrm{H} & -0.002996 \\ 137 & \mathrm{H} & 0.000045 \\ 138 & \mathrm{H} & -0.000024 \\ 139 & \mathrm{H} & -0.000053 \\ 140 & \mathrm{H} & 0.000121 \\ 141 & \mathrm{H} & 0.000003 \\ 142 & \mathrm{H} & 0.000018 \\ 143 & \mathrm{H} & -0.000031 \\ 144 & \mathrm{H} & -0.000041 \\ 145 & \mathrm{H} & 0.000004 \\ 146 & \mathrm{H} & 0.000023 \\ 147 & \mathrm{H} & -0.000056 \\ 148 & \mathrm{H} & -0.000024 \\ 149 & \mathrm{H} & 0.000045 \\ 150 & \mathrm{H} & -0.000034 \\ 151 & \mathrm{H} & 0.000530 \\ 152 & \mathrm{H} & 0.000077 \\ 153 & \mathrm{H} & 0.000059 \\ 154 & \mathrm{H} & 0.000609 \\ 155 & \mathrm{H} & -0.000093 \\ 156 & \mathrm{H} & -0.000019 \\ 157 & \mathrm{H} & 0.000112 \\ 158 & \mathrm{H} & -0.000052 \\ 159 & \mathrm{H} & 0.000002 \\ 160 & \mathrm{H} & -0.000044 \\ 161 & \mathrm{H} & 0.000038 \\ 162 & \mathrm{H} & 0.000019 \\ 163 & \mathrm{H} & -0.000036 \\ 164 & \mathrm{H} & -0.000001 \\ 165 & \mathrm{H} & 0.000045 \\ 166 & \mathrm{H} & -0.000024 \\ 167 & \mathrm{H} & -0.000057 \\ 168 & \mathrm{H} & 0.000058 \\ 169 & \mathrm{H} & -0.000094 \\ & \mathrm{H} & 0.000614 \\ & \mathrm{H} & -0.0000259\end{array}$




\begin{tabular}{|c|c|c|}
\hline & $\mathrm{H}$ & \\
\hline & $\mathrm{H}$ & \\
\hline & $\mathrm{H}$ & 0.000058 \\
\hline & $\mathrm{H}$ & 112 \\
\hline & $\mathrm{H}$ & \\
\hline & $\mathrm{H}$ & 754 \\
\hline & $\mathrm{H}$ & -0.0 \\
\hline & $\mathrm{H}$ & -0.0 \\
\hline & $\mathrm{H}$ & \\
\hline & $\mathrm{H}$ & 0.00 \\
\hline & $\mathrm{H}$ & -0.00 \\
\hline & $\mathrm{H}$ & -0.0 \\
\hline & $\mathrm{H}$ & 0.0 \\
\hline & $\mathrm{H}$ & 0.00 \\
\hline & $\mathrm{H}$ & -0.000285 \\
\hline & $\mathrm{H}$ & \\
\hline & $\mathrm{H}$ & 00001 \\
\hline & $\mathrm{H}$ & 0.00 \\
\hline & $\mathrm{H}$ & 0.0 \\
\hline & $\mathrm{H}$ & \\
\hline & $\mathrm{H}$ & -0.00 \\
\hline & $\mathrm{H}$ & -0.00 \\
\hline 7 & $\mathrm{H}$ & -0.0 \\
\hline & $\mathrm{H}$ & 0.00 \\
\hline & $\mathrm{H}$ & 0.0 \\
\hline 0 & $\mathrm{H}$ & 0.00 \\
\hline & $\mathrm{H}$ & -0.0 \\
\hline & $\mathrm{H}$ & -0.0 \\
\hline 03 & $\mathrm{H}$ & -0.000064 \\
\hline & $\mathrm{H}$ & 9423 \\
\hline & $\mathrm{H}$ & -0.000017 \\
\hline & $\mathrm{H}$ & -0.00 \\
\hline 207 & $\mathrm{H}$ & 0.000447 \\
\hline & $\mathrm{H}$ & 0.00000 \\
\hline & $\mathrm{H}$ & 0.000000 \\
\hline 10 & $\mathrm{H}$ & 0.000004 \\
\hline 11 & $\mathrm{H}$ & -0.000002 \\
\hline & $\mathrm{H}$ & -0.000007 \\
\hline & $\mathrm{H}$ & 0.000046 \\
\hline & $\mathrm{H}$ & -0.000004 \\
\hline & $\mathrm{H}$ & 0.000058 \\
\hline & $\mathrm{H}$ & 0.000002 \\
\hline & $\mathrm{H}$ & -0.000064 \\
\hline & $\mathrm{H}$ & 0.00042 \\
\hline
\end{tabular}




$$
\begin{array}{ccc}
219 & \mathrm{H} & -0.000026 \\
220 & \mathrm{H} & 0.000043 \\
221 & \mathrm{H} & -0.000038 \\
222 & \mathrm{H} & 0.000025 \\
223 & \mathrm{H} & -0.000017 \\
224 & \mathrm{H} & 0.000449 \\
225 & \mathrm{H} & -0.000076 \\
226 & \mathrm{H} & -0.000544 \\
227 & \mathrm{H} & -0.000583 \\
228 & \mathrm{H} & -0.000935
\end{array}
$$

\section{9b}

Energy (with Zero Point Energy correction $)=-4563.065791(-4561.126387)$ Hartree Free energy $(298 \mathrm{~K})=-4561.274991$ Hartree

Zero number of imaginary frequency

01

$\begin{array}{rrc}-11.88812800 & 1.54074600 & 1.33040600 \\ -11.68894200 & 0.30054300 & 0.70658200 \\ -10.37266500 & -0.09194400 & 0.32829300 \\ -9.29416700 & 0.81715000 & 0.48965200 \\ -9.54544600 & 2.01841000 & 1.17117400 \\ -10.82421100 & 2.39662500 & 1.61276100 \\ -10.15169500 & -1.39459300 & -0.22421700 \\ -8.83353600 & -1.86265500 & -0.46827600 \\ -7.68370200 & -0.99733700 & -0.11773100 \\ -7.94011900 & 0.41636200 & 0.04531000 \\ -11.26478500 & -2.23401900 & -0.51810500 \\ -11.05133700 & -3.47266800 & -1.13999800 \\ -9.77370700 & -3.90042900 & -1.49872900 \\ -8.68642500 & -3.08608800 & -1.14104000 \\ -12.78592900 & -0.58858600 & 0.43772500 \\ -12.58585200 & -1.78897000 & -0.16732200 \\ -6.36225300 & -1.46866000 & 0.00326200 \\ -5.27622800 & -0.54071300 & -0.00616000 \\ -5.54610000 & 0.85197700 & -0.25129400 \\ -6.88810100 & 1.32883400 & -0.16599400 \\ -3.92077200 & -0.96235900 & 0.16783100 \\ -2.87714700 & -0.08964600 & -0.02702100 \\ -3.15487700 & 1.27275100 & -0.41837700 \\ -4.44380300 & 1.72321800 & -0.52122700 \\ -0.12662800 & 3.55875300 & -1.05139900 \\ -1.51069200 & 3.23203900 & -0.97593400 \\ -1.86970000 & 1.95629400 & -0.60694000 \\ -0.85157000 & 0.99840900 & -0.31098600 \\ 0.53831000 & 1.30776100 & -0.38137300 \\ & & \end{array}$




\begin{tabular}{|c|c|c|c|}
\hline $\mathrm{C}$ & 0.86679900 & 2.64354000 & -0.76783800 \\
\hline $\mathrm{C}$ & -1.41814700 & -0.24885400 & 0.04638200 \\
\hline $\mathrm{C}$ & -0.54613200 & -1.31456500 & 0.37007200 \\
\hline $\mathrm{C}$ & 0.84377100 & -1.00519200 & 0.29977700 \\
\hline $\mathrm{C}$ & 1.41029600 & 0.24215100 & -0.05733100 \\
\hline $\mathrm{C}$ & 2.86923800 & 0.08326200 & 0.01690600 \\
\hline $\mathrm{C}$ & 3.14716000 & -1.27917600 & 0.40797900 \\
\hline $\mathrm{C}$ & 4.43616100 & -1.72923800 & 0.51158300 \\
\hline $\mathrm{C}$ & 5.53850100 & -0.85736600 & 0.24345600 \\
\hline $\mathrm{C}$ & 5.26806700 & 0.53535500 & -0.00089800 \\
\hline $\mathrm{C}$ & 3.91265200 & 0.95648100 & -0.17639900 \\
\hline $\mathrm{C}$ & 6.88100100 & -1.33329600 & 0.15937000 \\
\hline $\mathrm{C}$ & 7.93324500 & -0.42012700 & -0.04919900 \\
\hline $\mathrm{C}$ & 7.67503100 & 0.99325100 & 0.11431200 \\
\hline $\mathrm{C}$ & 6.35347800 & 1.46382600 & -0.00830200 \\
\hline $\mathrm{C}$ & 9.28866300 & -0.81898100 & -0.49228900 \\
\hline $\mathrm{C}$ & 10.36494900 & 0.09353900 & -0.33340400 \\
\hline $\mathrm{C}$ & 10.14181800 & 1.39558800 & 0.21971200 \\
\hline $\mathrm{C}$ & 8.82304100 & 1.86016500 & 0.46605700 \\
\hline $\mathrm{C}$ & 11.25309000 & 2.23789200 & 0.51241500 \\
\hline $\mathrm{C}$ & 11.03734500 & 3.47508100 & 1.13637200 \\
\hline $\mathrm{C}$ & 9.75921300 & 3.89872700 & 1.49832300 \\
\hline $\mathrm{C}$ & 8.67358400 & 3.08207100 & 1.14108300 \\
\hline $\mathrm{C}$ & 9.54357000 & -2.02145500 & -1.17099100 \\
\hline $\mathrm{C}$ & 10.82280200 & -2.39550900 & -1.61455200 \\
\hline $\mathrm{C}$ & 11.88392200 & -1.53438000 & -1.33780400 \\
\hline $\mathrm{C}$ & 11.68181800 & -0.29466200 & -0.71436300 \\
\hline $\mathrm{C}$ & 12.77674400 & 0.59784400 & -0.44810600 \\
\hline $\mathrm{C}$ & 12.57467200 & 1.79717900 & 0.15823500 \\
\hline $\mathrm{C}$ & -7.13681900 & 2.78544500 & -0.40454600 \\
\hline $\mathrm{C}$ & -11.08224800 & 3.72013500 & 2.35984200 \\
\hline $\mathrm{C}$ & -11.90648100 & 4.65763800 & 1.44513700 \\
\hline $\mathrm{C}$ & -9.77919600 & 4.44419200 & 2.74799000 \\
\hline $\mathrm{C}$ & -11.87465200 & 3.44709400 & 3.66018600 \\
\hline $\mathrm{C}$ & -9.59660200 & -5.23803200 & -2.24426900 \\
\hline $\mathrm{C}$ & -8.14002400 & -5.48601300 & -2.67922500 \\
\hline $\mathrm{C}$ & -10.47826700 & -5.24929000 & -3.51567700 \\
\hline $\mathrm{C}$ & -10.02799900 & -6.39526500 & -1.31183900 \\
\hline $\mathrm{C}$ & -6.05546900 & -2.91389300 & 0.24336700 \\
\hline $\mathrm{C}$ & 6.04631600 & 2.90903000 & -0.24834100 \\
\hline $\mathrm{C}$ & 7.13009300 & -2.79008000 & 0.39556800 \\
\hline $\mathrm{C}$ & 9.57983300 & 5.23450600 & 2.24655500 \\
\hline $\mathrm{C}$ & 10.00655900 & 6.39456000 & 1.31549000 \\
\hline $\mathrm{C}$ & 10.46366400 & 5.24578700 & 3.51646300 \\
\hline
\end{tabular}




\begin{tabular}{|c|c|c|c|}
\hline $\mathrm{C}$ & 8.12334500 & 5.47778900 & 2.68447700 \\
\hline $\mathrm{C}$ & 11.08596200 & -3.71524800 & -2.36650400 \\
\hline $\mathrm{C}$ & 9.78903600 & -4.47501000 & -2.70245200 \\
\hline $\mathrm{C}$ & 11.96903600 & -4.63007500 & -1.48446700 \\
\hline $\mathrm{C}$ & 11.82074300 & -3.42204100 & -3.69618300 \\
\hline $\mathrm{C}$ & -6.56761400 & 3.79225100 & 0.39352300 \\
\hline $\mathrm{C}$ & -6.77780400 & 5.13972000 & 0.11100900 \\
\hline $\mathrm{C}$ & -7.56095900 & 5.55464600 & -0.98071100 \\
\hline $\mathrm{C}$ & -8.12562800 & 4.54492700 & -1.77226000 \\
\hline $\mathrm{C}$ & -7.91793700 & 3.19317900 & -1.49314400 \\
\hline $\mathrm{C}$ & -5.22594300 & -3.66449300 & -0.60108100 \\
\hline $\mathrm{C}$ & -4.91652900 & -4.99747300 & -0.31814200 \\
\hline $\mathrm{C}$ & -5.41800800 & -5.64145800 & 0.82168500 \\
\hline $\mathrm{C}$ & -6.24816400 & -4.88164900 & 1.66467900 \\
\hline $\mathrm{C}$ & -6.55935900 & -3.55439500 & 1.38699300 \\
\hline $\mathrm{C}$ & 7.91316600 & -3.19823300 & 1.48739900 \\
\hline $\mathrm{C}$ & 8.12143500 & -4.54657600 & 1.75936500 \\
\hline $\mathrm{C}$ & 7.56118300 & -5.55991300 & 0.96149600 \\
\hline $\mathrm{C}$ & 6.77866500 & -5.14727700 & -0.12583200 \\
\hline $\mathrm{C}$ & 6.56537500 & -3.79440100 & -0.40223800 \\
\hline $\mathrm{C}$ & 6.55139100 & 3.55012000 & -1.39110800 \\
\hline $\mathrm{C}$ & 6.23980300 & 4.87726300 & -1.66890000 \\
\hline $\mathrm{C}$ & 5.40805200 & 5.63638500 & -0.82687000 \\
\hline $\mathrm{C}$ & 4.90540600 & 4.99182400 & 0.31211900 \\
\hline $\mathrm{C}$ & 5.21520700 & 3.65895500 & 0.59514500 \\
\hline $\mathrm{C}$ & -7.75463500 & 7.05458100 & -1.26685000 \\
\hline $\mathrm{C}$ & -8.65236400 & 7.30546900 & -2.49328900 \\
\hline $\mathrm{C}$ & -6.37717600 & 7.70652200 & -1.53611700 \\
\hline $\mathrm{C}$ & -8.41122000 & 7.73621600 & -0.04287800 \\
\hline $\mathrm{C}$ & -5.09096900 & -7.10368000 & 1.17328300 \\
\hline $\mathrm{C}$ & -4.18527900 & -7.77085400 & 0.12088500 \\
\hline $\mathrm{C}$ & -6.40239000 & -7.91954900 & 1.26659700 \\
\hline $\mathrm{C}$ & -4.36201000 & -7.15187500 & 2.53755600 \\
\hline $\mathrm{C}$ & 5.08058600 & 7.09848200 & -1.17858300 \\
\hline $\mathrm{C}$ & 4.17275600 & 7.76474800 & -0.12745200 \\
\hline $\mathrm{C}$ & 4.35377000 & 7.14659900 & -2.54400000 \\
\hline $\mathrm{C}$ & 6.39160900 & 7.91525000 & -1.26957300 \\
\hline $\mathrm{C}$ & 7.81027100 & -7.03950300 & 1.30435500 \\
\hline $\mathrm{C}$ & 9.33073300 & -7.32609800 & 1.28823400 \\
\hline $\mathrm{C}$ & 7.13207400 & -7.99486700 & 0.30419300 \\
\hline $\mathrm{C}$ & 7.24935600 & -7.34015000 & 2.71500000 \\
\hline $\mathrm{C}$ & -0.87458400 & -2.65046600 & 0.75615100 \\
\hline $\mathrm{C}$ & 0.11888200 & -3.56567300 & 1.03958800 \\
\hline $\mathrm{C}$ & 1.50293900 & -3.23882900 & 0.96448100 \\
\hline
\end{tabular}


C

$\mathrm{H}$

$\mathrm{H}$

$\mathrm{H}$

$\mathrm{H}$

$\mathrm{H}$

$\mathrm{H}$

$\mathrm{H}$

$\mathrm{H}$

$\mathrm{H}$

$\mathrm{H}$

$\mathrm{H}$

$\mathrm{H}$

$\mathrm{H}$

$\mathrm{H}$

$\mathrm{H}$

$\mathrm{H}$

$\mathrm{H}$

$\mathrm{H}$

$\mathrm{H}$

$\mathrm{H}$

$\mathrm{H}$

$\mathrm{H}$

$\mathrm{H}$

$\mathrm{H}$

$\mathrm{H}$

$\mathrm{H}$

$\mathrm{H}$

$\mathrm{H}$

$\mathrm{H}$

$\mathrm{H}$

$\mathrm{H}$

$\mathrm{H}$

$\mathrm{H}$

$\mathrm{H}$

$\mathrm{H}$

$\mathrm{H}$

$\mathrm{H}$

$\mathrm{H}$

$\mathrm{H}$

$\mathrm{H}$

$\mathrm{H}$

$\mathrm{H}$

$\mathrm{H}$

$\begin{array}{rrc}1.86198000 & -1.96300400 & 0.59581700 \\ -12.90267200 & 1.81247800 & 1.61031400 \\ -8.71441000 & 2.67217900 & 1.38083100 \\ -11.91948900 & -4.09004300 & -1.35646300 \\ -7.69333900 & -3.40605300 & -1.41177400 \\ -13.78719700 & -0.27027100 & 0.71849300 \\ -13.42485400 & -2.44569700 & -0.38520600 \\ -3.73505700 & -1.99092800 & 0.44207600 \\ -4.63876800 & 2.75328900 & -0.79402900 \\ 0.15432400 & 4.56713600 & -1.34405200 \\ -2.25546800 & 3.98840600 & -1.20966300 \\ 1.90450300 & 2.94944700 & -0.84033200 \\ 4.63139100 & -2.75944900 & 0.78354900 \\ 3.72681800 & 1.98513500 & -0.45020300 \\ 11.90417900 & 4.09460500 & 1.35197900 \\ 7.67999500 & 3.39885800 & 1.41375900 \\ 8.71493000 & -2.67922900 & -1.37724500 \\ 12.89846500 & -1.80207900 & -1.62192300 \\ 13.77823600 & 0.28252600 & -0.73143300 \\ 13.41223100 & 2.45622300 & 0.37466100 \\ -11.35954500 & 4.87823100 & 0.52122700 \\ -12.86612400 & 4.20860300 & 1.16642200 \\ -12.11479300 & 5.60676700 & 1.95499400 \\ -9.19254400 & 4.73855200 & 1.87209300 \\ -9.14567400 & 3.82044400 & 3.38946300 \\ -10.01958900 & 5.35654200 & 3.30615100 \\ -12.84782100 & 2.98575500 & 3.46408000 \\ -11.31638300 & 2.77947800 & 4.32642200 \\ -12.05721300 & 4.38654500 & 4.19610600 \\ -7.45910500 & -5.55257100 & -1.82466600 \\ -8.07798100 & -6.43348200 & -3.22719900 \\ -7.77533800 & -4.69491000 & -3.34473900 \\ -10.19573400 & -4.43726100 & -4.19540900 \\ -10.35804200 & -6.19854500 & -4.05207500 \\ -11.54165100 & -5.13422800 & -3.28223500 \\ -11.07260200 & -6.29478500 & -0.99769900 \\ -9.92339900 & -7.35997500 & -1.82417900 \\ -9.40783200 & -6.41924700 & -0.40847200 \\ 9.38483100 & 6.41847700 & 0.41319700 \\ 9.90022900 & 7.35806500 & 1.82973700 \\ 11.05090700 & 6.29745600 & 0.99943800 \\ 10.18440500 & 4.43182400 & 4.19523400 \\ 11.52693400 & 5.13388200 & 3.28097400 \\ 10.34191400 & 6.19377500 & 4.05475600\end{array}$




\begin{tabular}{|c|c|c|c|}
\hline $\mathrm{H}$ & 7.76192400 & 4.68443500 & 3.34909700 \\
\hline $\mathrm{H}$ & 8.05973000 & 6.42402900 & 3.23438700 \\
\hline $\mathrm{H}$ & 7.44078800 & 5.54419900 & 1.83121000 \\
\hline $\mathrm{H}$ & 9.11830100 & -3.87525800 & -3.32868300 \\
\hline $\mathrm{H}$ & 10.03313600 & -5.38731800 & -3.25919300 \\
\hline $\mathrm{H}$ & 9.23998800 & -4.77250100 & -1.80336400 \\
\hline $\mathrm{H}$ & 12.92745600 & -4.15783500 & -1.24284300 \\
\hline $\mathrm{H}$ & 11.46450400 & -4.86527900 & -0.54035900 \\
\hline $\mathrm{H}$ & 12.18163100 & -5.57351400 & -2.00295700 \\
\hline $\mathrm{H}$ & 11.21770900 & -2.77370400 & -4.34215000 \\
\hline $\mathrm{H}$ & 12.01296100 & -4.35662900 & -4.23735900 \\
\hline $\mathrm{H}$ & 12.78492300 & -2.92902600 & -3.53547000 \\
\hline $\mathrm{H}$ & -5.95914700 & 3.51450600 & 1.25021100 \\
\hline $\mathrm{H}$ & -6.31774000 & 5.88060200 & 0.75948400 \\
\hline $\mathrm{H}$ & -8.73541900 & 4.80165700 & -2.63150300 \\
\hline $\mathrm{H}$ & -8.36812800 & 2.44013900 & -2.13372000 \\
\hline $\mathrm{H}$ & -4.82074600 & -3.20453800 & -1.49857700 \\
\hline $\mathrm{H}$ & -4.27328800 & -5.53100700 & -1.00940800 \\
\hline $\mathrm{H}$ & -6.65850600 & -5.32987500 & 2.56554000 \\
\hline $\mathrm{H}$ & -7.20019900 & -2.99911700 & 2.06595600 \\
\hline $\mathrm{H}$ & 8.36016100 & -2.44482700 & 2.12972900 \\
\hline $\mathrm{H}$ & 8.73050800 & -4.81104900 & 2.61955400 \\
\hline $\mathrm{H}$ & 6.32098300 & -5.88148000 & -0.77989100 \\
\hline $\mathrm{H}$ & 5.95665100 & -3.51688300 & -1.25884000 \\
\hline $\mathrm{H}$ & 7.19348200 & 2.99539600 & -2.06934200 \\
\hline $\mathrm{H}$ & 6.65112300 & 5.32593900 & -2.56909000 \\
\hline $\mathrm{H}$ & 4.26086200 & 5.52479000 & 1.00261000 \\
\hline $\mathrm{H}$ & 4.80901000 & 3.19855000 & 1.49196200 \\
\hline $\mathrm{H}$ & -8.22428000 & 6.87858500 & -3.40734300 \\
\hline $\mathrm{H}$ & -8.76404700 & 8.38366900 & -2.65409900 \\
\hline $\mathrm{H}$ & -9.65570400 & 6.88619500 & -2.35693700 \\
\hline $\mathrm{H}$ & -5.89080100 & 7.24971900 & -2.40562200 \\
\hline $\mathrm{H}$ & -6.49469800 & 8.77859400 & -1.73641900 \\
\hline $\mathrm{H}$ & -5.70191700 & 7.59954200 & -0.68063300 \\
\hline $\mathrm{H}$ & -9.39487900 & 7.30107500 & 0.16756100 \\
\hline $\mathrm{H}$ & -7.80006500 & 7.63232600 & 0.85975700 \\
\hline $\mathrm{H}$ & -8.54711300 & 8.80827700 & -0.23118400 \\
\hline $\mathrm{H}$ & -4.65569400 & -7.79071000 & -0.86877200 \\
\hline $\mathrm{H}$ & -3.21943800 & -7.26079700 & 0.03032600 \\
\hline $\mathrm{H}$ & -3.98395900 & -8.80802600 & 0.41165000 \\
\hline $\mathrm{H}$ & -6.93900900 & -7.91050200 & 0.31093200 \\
\hline $\mathrm{H}$ & -7.07685500 & -7.52220500 & 2.03219400 \\
\hline $\mathrm{H}$ & -6.18459000 & -8.96330500 & 1.52422200 \\
\hline $\mathrm{H}$ & -3.42218700 & -6.58909400 & 2.50004600 \\
\hline
\end{tabular}




\begin{tabular}{lrrr}
$\mathrm{H}$ & -4.97281300 & -6.72739100 & 3.34111600 \\
$\mathrm{H}$ & -4.12699700 & -8.18850600 & 2.80852700 \\
$\mathrm{H}$ & 3.20712200 & 7.25400200 & -0.03859600 \\
$\mathrm{H}$ & 3.97119600 & 8.80186300 & -0.41825000 \\
$\mathrm{H}$ & 4.64156600 & 7.78465000 & 0.86296700 \\
$\mathrm{H}$ & 3.41424200 & 6.58322000 & -2.50814600 \\
$\mathrm{H}$ & 4.96611500 & 6.72273700 & -3.34671400 \\
$\mathrm{H}$ & 4.11853100 & 8.18315800 & -2.81504500 \\
$\mathrm{H}$ & 7.06754900 & 7.51856100 & -2.03421000 \\
$\mathrm{H}$ & 6.17354100 & 8.95893900 & -1.52724400 \\
$\mathrm{H}$ & 6.92671300 & 7.90627800 & -0.31305700 \\
$\mathrm{H}$ & 9.87056800 & -6.70765100 & 2.01283300 \\
$\mathrm{H}$ & 9.52333400 & -8.37657600 & 1.53854600 \\
$\mathrm{H}$ & 9.75684900 & -7.12947000 & 0.29770400 \\
$\mathrm{H}$ & 7.50440900 & -7.84887300 & -0.71620400 \\
$\mathrm{H}$ & 7.34047100 & -9.03300900 & 0.58639700 \\
$\mathrm{H}$ & 6.04339900 & -7.86904800 & 0.29267000 \\
$\mathrm{H}$ & 6.16974500 & -7.15547700 & 2.75519300 \\
$\mathrm{H}$ & 7.42487200 & -8.39007600 & 2.97985400 \\
$\mathrm{H}$ & 7.72286800 & -6.71900900 & 3.48244900 \\
$\mathrm{H}$ & -1.91227400 & -2.95651000 & 0.82828600 \\
$\mathrm{H}$ & -0.16205500 & -4.57416000 & 1.33190100 \\
$\mathrm{H}$ & 2.24773200 & -3.99516300 & 1.19826100 \\
& & & \\
$\mathrm{E}$ & & $\mathbf{9 b}$ & \\
\hline & & & \\
& & & \\
& & & \\
& & &
\end{tabular}

Energy (with Zero Point Energy correction $)=-4562.867469(-4560.927873)$ Hartree Free energy $(298 \mathrm{~K})=-4561.077171$ Hartree

Zero number of imaginary frequency

12

$\begin{array}{lrrr}\mathrm{C} & -11.83675900 & 1.53374600 & 1.39665800 \\ \mathrm{C} & -11.65282200 & 0.29838000 & 0.76148500 \\ \mathrm{C} & -10.34260900 & -0.09411800 & 0.36268700 \\ \mathrm{C} & -9.25992300 & 0.81751500 & 0.51620800 \\ \mathrm{C} & -9.49991600 & 2.02182400 & 1.20495900 \\ \mathrm{C} & -10.76831100 & 2.39330400 & 1.66969900 \\ \mathrm{C} & -10.13399400 & -1.38687200 & -0.20709300 \\ \mathrm{C} & -8.82027800 & -1.85471300 & -0.48123500 \\ \mathrm{C} & -7.67339300 & -0.98995500 & -0.14206500 \\ \mathrm{C} & -7.92335800 & 0.41691700 & 0.05558400 \\ \mathrm{C} & -11.25330900 & -2.22327500 & -0.49045600 \\ \mathrm{C} & -11.04768900 & -3.45370000 & -1.12853900 \\ \mathrm{C} & -9.77538000 & -3.88110700 & -1.51294300 \\ \mathrm{C} & -8.68126200 & -3.07331500 & -1.16411100 \\ \mathrm{C} & -12.75691100 & -0.58535200 & 0.50318500\end{array}$




\begin{tabular}{|c|c|c|c|}
\hline $\mathrm{C}$ & -12.56825400 & -1.78089400 & -0.11464600 \\
\hline $\mathrm{C}$ & -6.34714900 & -1.46294900 & -0.01785400 \\
\hline $\mathrm{C}$ & -5.26370600 & -0.53045900 & -0.02117800 \\
\hline $\mathrm{C}$ & -5.53224600 & 0.86461700 & -0.26132400 \\
\hline $\mathrm{C}$ & -6.86436500 & 1.34086200 & -0.16986900 \\
\hline $\mathrm{C}$ & -3.91677500 & -0.95001300 & 0.16300600 \\
\hline $\mathrm{C}$ & -2.86424500 & -0.07271700 & -0.03075100 \\
\hline $\mathrm{C}$ & -3.13954300 & 1.28660700 & -0.43420900 \\
\hline $\mathrm{C}$ & -4.42729500 & 1.73549300 & -0.54266200 \\
\hline $\mathrm{C}$ & -0.10882100 & 3.55677300 & -1.08149400 \\
\hline $\mathrm{C}$ & -1.48703400 & 3.23930800 & -1.00630300 \\
\hline $\mathrm{C}$ & -1.85391100 & 1.96228100 & -0.62568700 \\
\hline $\mathrm{C}$ & -0.84280400 & 1.00869900 & -0.32083200 \\
\hline $\mathrm{C}$ & 0.54445900 & 1.31081400 & -0.39099400 \\
\hline $\mathrm{C}$ & 0.88283300 & 2.63374100 & -0.78623700 \\
\hline $\mathrm{C}$ & -1.42261300 & -0.24025300 & 0.04761400 \\
\hline $\mathrm{C}$ & -0.55256900 & -1.31613000 & 0.38420000 \\
\hline $\mathrm{C}$ & 0.83471400 & -1.01396800 & 0.31420800 \\
\hline $\mathrm{C}$ & 1.41447700 & 0.23485200 & -0.05463400 \\
\hline $\mathrm{C}$ & 2.85606600 & 0.06741400 & 0.02395500 \\
\hline $\mathrm{C}$ & 3.13150500 & -1.29146800 & 0.42873000 \\
\hline $\mathrm{C}$ & 4.41933500 & -1.73983800 & 0.53864000 \\
\hline $\mathrm{C}$ & 5.52436400 & -0.86902300 & 0.25707000 \\
\hline $\mathrm{C}$ & 5.25540900 & 0.52562500 & 0.01483800 \\
\hline $\mathrm{C}$ & 3.90847300 & 0.94462700 & -0.17049600 \\
\hline $\mathrm{C}$ & 6.85683700 & -1.34478900 & 0.16715200 \\
\hline $\mathrm{C}$ & 7.91598700 & -0.42085400 & -0.05899600 \\
\hline $\mathrm{C}$ & 7.66484700 & 0.98623500 & 0.13577800 \\
\hline $\mathrm{C}$ & 6.33843200 & 1.45843100 & 0.01037800 \\
\hline $\mathrm{C}$ & 9.25324200 & -0.82123900 & -0.51861200 \\
\hline $\mathrm{C}$ & 10.33434900 & 0.09345700 & -0.37039600 \\
\hline $\mathrm{C}$ & 10.12459800 & 1.38725400 & 0.19660700 \\
\hline $\mathrm{C}$ & 8.81069000 & 1.85305400 & 0.47266200 \\
\hline $\mathrm{C}$ & 11.24275800 & 2.22668700 & 0.47570100 \\
\hline $\mathrm{C}$ & 11.03602300 & 3.45761200 & 1.11242300 \\
\hline $\mathrm{C}$ & 9.76376000 & 3.88273600 & 1.49958900 \\
\hline $\mathrm{C}$ & 8.67057500 & 3.07221600 & 1.15431300 \\
\hline $\mathrm{C}$ & 9.49557500 & -2.02842600 & -1.20194700 \\
\hline $\mathrm{C}$ & 10.76395800 & -2.39821600 & -1.66801000 \\
\hline $\mathrm{C}$ & 11.83031900 & -1.53366500 & -1.40290200 \\
\hline $\mathrm{C}$ & 11.64460700 & -0.29679600 & -0.77143100 \\
\hline $\mathrm{C}$ & 12.74728100 & 0.59035000 & -0.51873100 \\
\hline $\mathrm{C}$ & 12.55768000 & 1.78675100 & 0.09705900 \\
\hline $\mathrm{C}$ & -7.12866200 & 2.78939100 & -0.42001900 \\
\hline
\end{tabular}




\begin{tabular}{|c|c|c|c|}
\hline $\mathrm{C}$ & -11.02049000 & 3.70510100 & 2.43707500 \\
\hline $\mathrm{C}$ & -11.90186900 & 4.63241900 & 1.56591400 \\
\hline $\mathrm{C}$ & -9.71609400 & 4.45115500 & 2.77445400 \\
\hline $\mathrm{C}$ & -11.75152200 & 3.40143700 & 3.76662700 \\
\hline $\mathrm{C}$ & -9.61526500 & -5.20819100 & -2.27931600 \\
\hline $\mathrm{C}$ & -8.16218100 & -5.46508500 & -2.72053800 \\
\hline $\mathrm{C}$ & -10.50070700 & -5.18585300 & -3.54823600 \\
\hline $\mathrm{C}$ & -10.05880400 & -6.37479700 & -1.36404700 \\
\hline $\mathrm{C}$ & -6.04425500 & -2.90548600 & 0.22269800 \\
\hline $\mathrm{C}$ & 6.03504200 & 2.90043900 & -0.23294500 \\
\hline $\mathrm{C}$ & 7.12179400 & -2.79267600 & 0.41928900 \\
\hline $\mathrm{C}$ & 9.60271100 & 5.21034000 & 2.26484200 \\
\hline $\mathrm{C}$ & 10.04163200 & 6.37684500 & 1.34722900 \\
\hline $\mathrm{C}$ & 10.49124900 & 5.19123600 & 3.53165500 \\
\hline $\mathrm{C}$ & 8.15015500 & 5.46468100 & 2.70928500 \\
\hline $\mathrm{C}$ & 11.01904400 & -3.70998100 & -2.43450000 \\
\hline $\mathrm{C}$ & 9.72036000 & -4.48205600 & -2.73246800 \\
\hline $\mathrm{C}$ & 11.94074500 & -4.61799100 & -1.58509700 \\
\hline $\mathrm{C}$ & 11.70809400 & -3.39733200 & -3.78438700 \\
\hline $\mathrm{C}$ & -6.53723600 & 3.80921800 & 0.34592500 \\
\hline $\mathrm{C}$ & -6.76652000 & 5.15075600 & 0.05209500 \\
\hline $\mathrm{C}$ & -7.58612400 & 5.54554700 & -1.02056600 \\
\hline $\mathrm{C}$ & -8.17133500 & 4.52128300 & -1.77927100 \\
\hline $\mathrm{C}$ & -7.95198900 & 3.17525200 & -1.48660000 \\
\hline $\mathrm{C}$ & -5.19810300 & -3.65001600 & -0.61173200 \\
\hline $\mathrm{C}$ & -4.89985700 & -4.98627200 & -0.33248400 \\
\hline $\mathrm{C}$ & -5.42400300 & -5.63602000 & 0.79391800 \\
\hline $\mathrm{C}$ & -6.26817300 & -4.87917800 & 1.62729800 \\
\hline $\mathrm{C}$ & -6.57522000 & -3.55151600 & 1.35188200 \\
\hline $\mathrm{C}$ & 7.94902200 & -3.17547600 & 1.48885600 \\
\hline $\mathrm{C}$ & 8.16968000 & -4.51722300 & 1.77799400 \\
\hline $\mathrm{C}$ & 7.58751300 & -5.54776100 & 1.01709900 \\
\hline $\mathrm{C}$ & 6.76701800 & -5.15863000 & -0.05105900 \\
\hline $\mathrm{C}$ & 6.53406700 & -3.81251800 & -0.34268000 \\
\hline $\mathrm{C}$ & 6.56560700 & 3.54442700 & -1.36347100 \\
\hline $\mathrm{C}$ & 6.25784500 & 4.87136400 & -1.64160300 \\
\hline $\mathrm{C}$ & 5.41333600 & 5.62948100 & -0.80973900 \\
\hline $\mathrm{C}$ & 4.88964200 & 4.98178900 & 0.31806200 \\
\hline $\mathrm{C}$ & 5.18858900 & 3.64625900 & 0.60002100 \\
\hline $\mathrm{C}$ & -7.80026500 & 7.03947200 & -1.32050000 \\
\hline $\mathrm{C}$ & -8.73436200 & 7.26653300 & -2.52413000 \\
\hline $\mathrm{C}$ & -6.43513400 & 7.69700300 & -1.63643900 \\
\hline $\mathrm{C}$ & -8.42685500 & 7.73001500 & -0.08552000 \\
\hline $\mathrm{C}$ & -5.11200600 & -7.10256700 & 1.13879000 \\
\hline
\end{tabular}




\begin{tabular}{|c|c|c|c|}
\hline $\mathrm{C}$ & -4.18550200 & -7.76369600 & 0.10090500 \\
\hline $\mathrm{C}$ & -6.43066800 & -7.91078700 & 1.19364000 \\
\hline $\mathrm{C}$ & -4.41604900 & -7.16610300 & 2.51979400 \\
\hline $\mathrm{C}$ & 5.10039800 & 7.09509600 & -1.15770000 \\
\hline $\mathrm{C}$ & 4.17377300 & 7.75792600 & -0.12100700 \\
\hline $\mathrm{C}$ & 4.40402200 & 7.15519700 & -2.53865100 \\
\hline $\mathrm{C}$ & 6.41855000 & 7.90399300 & -1.21467100 \\
\hline $\mathrm{C}$ & 7.85792700 & -7.01918700 & 1.37579300 \\
\hline $\mathrm{C}$ & 9.37927100 & -7.29513900 & 1.30972100 \\
\hline $\mathrm{C}$ & 7.14984100 & -7.99508700 & 0.41715800 \\
\hline $\mathrm{C}$ & 7.34988600 & -7.29601800 & 2.81145700 \\
\hline $\mathrm{C}$ & -0.89093600 & -2.63889000 & 0.77997300 \\
\hline $\mathrm{C}$ & 0.10072900 & -3.56167200 & 1.07601100 \\
\hline $\mathrm{C}$ & 1.47892400 & -3.24407900 & 1.00118700 \\
\hline $\mathrm{C}$ & 1.84587000 & -1.96722600 & 0.62002300 \\
\hline $\mathrm{H}$ & -12.84524100 & 1.80493700 & 1.69675600 \\
\hline $\mathrm{H}$ & -8.66623200 & 2.67546200 & 1.40419400 \\
\hline $\mathrm{H}$ & -11.91824500 & -4.06865700 & -1.33971800 \\
\hline $\mathrm{H}$ & -7.69276100 & -3.39293600 & -1.45269300 \\
\hline $\mathrm{H}$ & -13.75244500 & -0.26738800 & 0.80184400 \\
\hline $\mathrm{H}$ & -13.41130000 & -2.43390500 & -0.32510300 \\
\hline $\mathrm{H}$ & -3.73403100 & -1.97737300 & 0.44383600 \\
\hline $\mathrm{H}$ & -4.62666900 & 2.76088500 & -0.82924900 \\
\hline $\mathrm{H}$ & 0.18024100 & 4.55963200 & -1.38242300 \\
\hline $\mathrm{H}$ & -2.22829600 & 3.99582300 & -1.24805500 \\
\hline $\mathrm{H}$ & 1.92088000 & 2.93658100 & -0.85999500 \\
\hline $\mathrm{H}$ & 4.61892200 & -2.76481400 & 0.82645400 \\
\hline $\mathrm{H}$ & 3.72565500 & 1.97152400 & -0.45291100 \\
\hline $\mathrm{H}$ & 11.90575200 & 4.07481600 & 1.32043300 \\
\hline $\mathrm{H}$ & 7.68200700 & 3.39010300 & 1.44461200 \\
\hline $\mathrm{H}$ & 8.66361900 & -2.68568000 & -1.39608500 \\
\hline $\mathrm{H}$ & 12.83849800 & -1.80250400 & -1.70627100 \\
\hline $\mathrm{H}$ & 13.74266800 & 0.27395200 & -0.81952700 \\
\hline $\mathrm{H}$ & 13.39978100 & 2.44217600 & 0.30375700 \\
\hline $\mathrm{H}$ & -11.40047700 & 4.87396000 & 0.62160800 \\
\hline $\mathrm{H}$ & -12.86501100 & 4.16985100 & 1.32475600 \\
\hline $\mathrm{H}$ & -12.10576900 & 5.57115900 & 2.09485300 \\
\hline $\mathrm{H}$ & -9.17113300 & 4.75863300 & 1.87596000 \\
\hline $\mathrm{H}$ & -9.04630900 & 3.83954700 & 3.39046900 \\
\hline $\mathrm{H}$ & -9.95075800 & 5.35752100 & 3.34348000 \\
\hline $\mathrm{H}$ & -12.72178400 & 2.92050300 & 3.60666300 \\
\hline $\mathrm{H}$ & -11.15148100 & 2.74194700 & 4.40388900 \\
\hline $\mathrm{H}$ & -11.93275000 & 4.33183900 & 4.31731900 \\
\hline $\mathrm{H}$ & -7.48041300 & -5.55052900 & -1.86778500 \\
\hline
\end{tabular}




\begin{tabular}{|c|c|c|c|}
\hline $\mathrm{H}$ & -8.11159100 & -6.40607600 & -3.27958500 \\
\hline $\mathrm{H}$ & -7.79047000 & -4.67110400 & -3.37888000 \\
\hline $\mathrm{H}$ & -10.21031000 & -4.36684000 & -4.21602900 \\
\hline $\mathrm{H}$ & -10.39349900 & -6.12733000 & -4.09970300 \\
\hline $\mathrm{H}$ & -11.56197300 & -5.06281800 & -3.30985000 \\
\hline $\mathrm{H}$ & -11.10196500 & -6.27032600 & -1.04727600 \\
\hline $\mathrm{H}$ & -9.96565100 & -7.33050100 & -1.89380600 \\
\hline $\mathrm{H}$ & -9.43799800 & -6.42255100 & -0.46199500 \\
\hline $\mathrm{H}$ & 9.41861000 & 6.42226200 & 0.44658700 \\
\hline $\mathrm{H}$ & 9.94770900 & 7.33294500 & 1.87613700 \\
\hline $\mathrm{H}$ & 11.08426700 & 6.27422500 & 1.02812500 \\
\hline $\mathrm{H}$ & 10.20409500 & 4.37241700 & 4.20108700 \\
\hline $\mathrm{H}$ & 11.55218200 & 5.07003300 & 3.29085000 \\
\hline $\mathrm{H}$ & 10.38348700 & 6.13313300 & 4.08229600 \\
\hline $\mathrm{H}$ & 7.78169100 & 4.67061400 & 3.36934800 \\
\hline $\mathrm{H}$ & 8.09895200 & 6.40614200 & 3.26748100 \\
\hline $\mathrm{H}$ & 7.46616400 & 5.54783400 & 1.85808600 \\
\hline $\mathrm{H}$ & 9.02495700 & -3.88982100 & -3.33887800 \\
\hline $\mathrm{H}$ & 9.95742400 & -5.39037300 & -3.29746800 \\
\hline $\mathrm{H}$ & 9.20258800 & -4.78796400 & -1.81736500 \\
\hline $\mathrm{H}$ & 12.90211900 & -4.13848900 & -1.37192700 \\
\hline $\mathrm{H}$ & 11.47027700 & -4.86638400 & -0.62686600 \\
\hline $\mathrm{H}$ & 12.14682400 & -5.55439000 & -2.11729400 \\
\hline $\mathrm{H}$ & 11.07750500 & -2.75363100 & -4.40817300 \\
\hline $\mathrm{H}$ & 11.89510600 & -4.32603500 & -4.33610600 \\
\hline $\mathrm{H}$ & 12.67118100 & -2.89427100 & -3.65085300 \\
\hline $\mathrm{H}$ & -5.90707200 & 3.54766900 & 1.19210000 \\
\hline $\mathrm{H}$ & -6.29512700 & 5.90361100 & 0.67749600 \\
\hline $\mathrm{H}$ & -8.80911700 & 4.76373500 & -2.62170600 \\
\hline $\mathrm{H}$ & -8.42119800 & 2.41255500 & -2.10143300 \\
\hline $\mathrm{H}$ & -4.78318400 & -3.18869400 & -1.50426700 \\
\hline $\mathrm{H}$ & -4.25067800 & -5.51965200 & -1.01794100 \\
\hline $\mathrm{H}$ & -6.69589400 & -5.33274700 & 2.51673000 \\
\hline $\mathrm{H}$ & -7.23024900 & -3.00171500 & 2.02144800 \\
\hline $\mathrm{H}$ & 8.41589300 & -2.41044900 & 2.10250700 \\
\hline $\mathrm{H}$ & 8.80852100 & -4.76403900 & 2.62099500 \\
\hline $\mathrm{H}$ & 6.29671200 & -5.90728000 & -0.67876600 \\
\hline $\mathrm{H}$ & 5.90253000 & -3.55392100 & -1.18876400 \\
\hline $\mathrm{H}$ & 7.22086300 & 2.99359500 & -2.03196700 \\
\hline $\mathrm{H}$ & 6.68524300 & 5.32330400 & -2.53201800 \\
\hline $\mathrm{H}$ & 4.24023200 & 5.51623800 & 1.00246800 \\
\hline $\mathrm{H}$ & 4.77392300 & 3.18654200 & 1.49350600 \\
\hline $\mathrm{H}$ & -8.32973300 & 6.83134900 & -3.44494600 \\
\hline $\mathrm{H}$ & -8.85789700 & 8.34122200 & -2.69574700 \\
\hline
\end{tabular}




\begin{tabular}{|c|c|c|c|}
\hline $\mathrm{H}$ & -9.73115900 & 6.84431600 & -2.35355400 \\
\hline $\mathrm{H}$ & -5.96947200 & 7.23381000 & -2.51391700 \\
\hline $\mathrm{H}$ & -6.56852500 & 8.76449000 & -1.84758300 \\
\hline $\mathrm{H}$ & -5.73555400 & 7.60936300 & -0.79826500 \\
\hline $\mathrm{H}$ & -9.40063500 & 7.29058600 & 0.15885800 \\
\hline $\mathrm{H}$ & -7.78877500 & 7.64517900 & 0.80040600 \\
\hline $\mathrm{H}$ & -8.57763600 & 8.79744800 & -0.28483300 \\
\hline $\mathrm{H}$ & -4.63342400 & -7.77527400 & -0.89919000 \\
\hline $\mathrm{H}$ & -3.21513500 & -7.25769700 & 0.03649100 \\
\hline $\mathrm{H}$ & -3.99486700 & -8.80348100 & 0.38755100 \\
\hline $\mathrm{H}$ & -6.94546700 & -7.88978400 & 0.22637400 \\
\hline $\mathrm{H}$ & -7.11984800 & -7.51951800 & 1.94913500 \\
\hline $\mathrm{H}$ & -6.22322600 & -8.95767100 & 1.44444500 \\
\hline $\mathrm{H}$ & -3.47165400 & -6.60941900 & 2.51058200 \\
\hline $\mathrm{H}$ & -5.04303900 & -6.74784600 & 3.31400000 \\
\hline $\mathrm{H}$ & -4.19358800 & -8.20638800 & 2.78476500 \\
\hline $\mathrm{H}$ & 3.20371100 & 7.25151300 & -0.05526800 \\
\hline $\mathrm{H}$ & 3.98246500 & 8.79698000 & -0.40984500 \\
\hline $\mathrm{H}$ & 4.62193500 & 7.77191200 & 0.87895200 \\
\hline $\mathrm{H}$ & 3.45998500 & 6.59793500 & -2.52797900 \\
\hline $\mathrm{H}$ & 5.03106700 & 6.73560900 & -3.33211200 \\
\hline $\mathrm{H}$ & 4.18082600 & 8.19476100 & -2.80582300 \\
\hline $\mathrm{H}$ & 7.10776900 & 7.51149000 & -1.96948900 \\
\hline $\mathrm{H}$ & 6.21040100 & 8.95019600 & -1.46772400 \\
\hline $\mathrm{H}$ & 6.93361600 & 7.88543100 & -0.24749700 \\
\hline $\mathrm{H}$ & 9.94123800 & -6.66291700 & 2.00499700 \\
\hline $\mathrm{H}$ & 9.58610400 & -8.33968900 & 1.57047500 \\
\hline $\mathrm{H}$ & 9.76879700 & -7.11447300 & 0.30129600 \\
\hline $\mathrm{H}$ & 7.48516500 & -7.86734700 & -0.61840800 \\
\hline $\mathrm{H}$ & 7.37441000 & -9.02621000 & 0.71039500 \\
\hline $\mathrm{H}$ & 6.06043300 & -7.87669800 & 0.44294200 \\
\hline $\mathrm{H}$ & 6.27115800 & -7.11761800 & 2.88842400 \\
\hline $\mathrm{H}$ & 7.54122800 & -8.33993900 & 3.08627800 \\
\hline $\mathrm{H}$ & 7.84812400 & -6.66101400 & 3.55137000 \\
\hline $\mathrm{H}$ & -1.92898600 & -2.94173000 & 0.85371100 \\
\hline $\mathrm{H}$ & -0.18834300 & -4.56439500 & 1.37738200 \\
\hline $\mathrm{H}$ & 2.22018100 & -4.00035500 & 1.24368800 \\
\hline
\end{tabular}

Mulliken spin densities:

$\begin{array}{ccc}1 & \mathrm{C} & 0.041680 \\ 2 & \mathrm{C} & -0.015482 \\ 3 & \mathrm{C} & 0.021373 \\ 4 & \mathrm{C} & 0.006465 \\ 5 & \mathrm{C} & 0.017303 \\ 6 & \mathrm{C} & -0.008354\end{array}$




\begin{tabular}{|c|c|c|}
\hline 7 & $\mathrm{C}$ & 0.000398 \\
\hline 8 & $\mathrm{C}$ & 0.020244 \\
\hline 9 & $\mathrm{C}$ & -0.037222 \\
\hline 10 & $\mathrm{C}$ & 0.048693 \\
\hline 11 & $\mathrm{C}$ & 0.007598 \\
\hline 12 & $\mathrm{C}$ & -0.010184 \\
\hline 13 & $\mathrm{C}$ & 0.013889 \\
\hline 14 & $\mathrm{C}$ & -0.014085 \\
\hline 15 & $\mathrm{C}$ & 0.008801 \\
\hline 16 & $\mathrm{C}$ & -0.008032 \\
\hline 17 & $\mathrm{C}$ & 0.106676 \\
\hline 18 & $\mathrm{C}$ & -0.041668 \\
\hline 19 & $\mathrm{C}$ & -0.008161 \\
\hline 20 & $\mathrm{C}$ & 0.051884 \\
\hline 21 & $\mathrm{C}$ & 0.134635 \\
\hline 22 & $\mathrm{C}$ & -0.030692 \\
\hline 23 & $\mathrm{C}$ & -0.018425 \\
\hline 24 & $\mathrm{C}$ & 0.057172 \\
\hline 25 & $\mathrm{C}$ & 0.001166 \\
\hline 26 & $\mathrm{C}$ & 0.017066 \\
\hline 27 & $\mathrm{C}$ & 0.019847 \\
\hline 28 & $\mathrm{C}$ & -0.013182 \\
\hline 29 & $\mathrm{C}$ & -0.014633 \\
\hline 30 & $\mathrm{C}$ & 0.036413 \\
\hline 31 & $\mathrm{C}$ & 0.102426 \\
\hline 32 & $\mathrm{C}$ & -0.014720 \\
\hline 33 & $\mathrm{C}$ & -0.013162 \\
\hline 34 & $\mathrm{C}$ & 0.102278 \\
\hline 35 & $\mathrm{C}$ & -0.030759 \\
\hline 36 & $\mathrm{C}$ & -0.018434 \\
\hline 37 & $\mathrm{C}$ & 0.057244 \\
\hline 38 & $\mathrm{C}$ & -0.008142 \\
\hline 39 & $\mathrm{C}$ & -0.041783 \\
\hline 40 & $\mathrm{C}$ & 0.134954 \\
\hline 41 & $\mathrm{C}$ & 0.051824 \\
\hline 42 & $\mathrm{C}$ & 0.048776 \\
\hline 43 & $\mathrm{C}$ & -0.037293 \\
\hline 44 & $\mathrm{C}$ & 0.106862 \\
\hline 45 & $\mathrm{C}$ & 0.006354 \\
\hline 46 & $\mathrm{C}$ & 0.021434 \\
\hline 47 & $\mathrm{C}$ & 0.000349 \\
\hline 48 & $\mathrm{C}$ & 0.020281 \\
\hline 49 & $\mathrm{C}$ & 0.007599 \\
\hline 50 & $\mathrm{C}$ & -0.010165 \\
\hline
\end{tabular}




\begin{tabular}{|c|c|c|}
\hline 51 & $\mathrm{C}$ & 0.013893 \\
\hline 52 & $\mathrm{C}$ & -0.014089 \\
\hline 53 & $\mathrm{C}$ & 0.017195 \\
\hline 54 & $\mathrm{C}$ & -0.008291 \\
\hline 55 & $\mathrm{C}$ & 0.041593 \\
\hline 56 & $\mathrm{C}$ & -0.015462 \\
\hline 57 & $\mathrm{C}$ & 0.008783 \\
\hline 58 & $\mathrm{C}$ & -0.008028 \\
\hline 59 & $\mathrm{C}$ & -0.002485 \\
\hline 60 & $\mathrm{C}$ & 0.000331 \\
\hline 61 & $\mathrm{C}$ & 0.000152 \\
\hline 62 & $\mathrm{C}$ & -0.000044 \\
\hline 63 & $\mathrm{C}$ & 0.000005 \\
\hline 64 & $\mathrm{C}$ & -0.000807 \\
\hline 65 & $\mathrm{C}$ & 0.000013 \\
\hline 66 & $\mathrm{C}$ & 0.000328 \\
\hline 67 & $\mathrm{C}$ & 0.000474 \\
\hline 68 & $\mathrm{C}$ & -0.008565 \\
\hline 69 & $\mathrm{C}$ & -0.008575 \\
\hline 70 & $\mathrm{C}$ & -0.002414 \\
\hline 71 & $\mathrm{C}$ & -0.000808 \\
\hline 72 & $\mathrm{C}$ & 0.000474 \\
\hline 73 & $\mathrm{C}$ & 0.000328 \\
\hline 74 & $\mathrm{C}$ & 0.000013 \\
\hline 75 & $\mathrm{C}$ & 0.000325 \\
\hline 76 & $\mathrm{C}$ & -0.000039 \\
\hline 77 & $\mathrm{C}$ & 0.000143 \\
\hline 78 & $\mathrm{C}$ & 0.000016 \\
\hline 79 & $\mathrm{C}$ & 0.005221 \\
\hline 80 & $\mathrm{C}$ & -0.002868 \\
\hline 81 & $\mathrm{C}$ & 0.006755 \\
\hline 82 & $\mathrm{C}$ & -0.002359 \\
\hline 83 & $\mathrm{C}$ & 0.005589 \\
\hline 84 & $\mathrm{C}$ & 0.008256 \\
\hline 85 & $\mathrm{C}$ & -0.003736 \\
\hline 86 & $\mathrm{C}$ & 0.007610 \\
\hline 87 & $\mathrm{C}$ & -0.002277 \\
\hline 88 & $\mathrm{C}$ & 0.006247 \\
\hline 89 & $\mathrm{C}$ & 0.005021 \\
\hline 90 & $\mathrm{C}$ & -0.002271 \\
\hline 91 & $\mathrm{C}$ & 0.006761 \\
\hline 92 & $\mathrm{C}$ & -0.002899 \\
\hline 93 & $\mathrm{C}$ & 0.005717 \\
\hline 94 & $\mathrm{C}$ & 0.006253 \\
\hline
\end{tabular}




\begin{tabular}{|c|c|c|}
\hline 95 & $\mathrm{C}$ & -0.002278 \\
\hline 96 & $\mathrm{C}$ & 0.007616 \\
\hline 97 & $\mathrm{C}$ & -0.003738 \\
\hline 98 & $\mathrm{C}$ & 0.008262 \\
\hline 99 & $\mathrm{C}$ & -0.000399 \\
\hline 100 & $\mathrm{C}$ & 0.000013 \\
\hline 101 & $\mathrm{C}$ & 0.000241 \\
\hline 102 & $\mathrm{C}$ & 0.000215 \\
\hline 103 & $\mathrm{C}$ & -0.000473 \\
\hline 104 & $\mathrm{C}$ & 0.000012 \\
\hline 105 & $\mathrm{C}$ & 0.000224 \\
\hline 106 & $\mathrm{C}$ & 0.000263 \\
\hline 107 & $\mathrm{C}$ & -0.000473 \\
\hline 108 & $\mathrm{C}$ & 0.000012 \\
\hline 109 & $\mathrm{C}$ & 0.000264 \\
\hline 110 & $\mathrm{C}$ & 0.000224 \\
\hline 111 & $\mathrm{C}$ & -0.000407 \\
\hline 112 & $\mathrm{C}$ & 0.000206 \\
\hline 113 & $\mathrm{C}$ & 0.000012 \\
\hline 114 & $\mathrm{C}$ & 0.000251 \\
\hline 115 & $\mathrm{C}$ & 0.036593 \\
\hline 116 & $\mathrm{C}$ & 0.001076 \\
\hline 117 & $\mathrm{C}$ & 0.017243 \\
\hline 118 & $\mathrm{C}$ & 0.019779 \\
\hline 119 & $\mathrm{H}$ & -0.001811 \\
\hline 120 & $\mathrm{H}$ & -0.000911 \\
\hline 121 & $\mathrm{H}$ & 0.000424 \\
\hline 122 & $\mathrm{H}$ & 0.000484 \\
\hline 123 & $\mathrm{H}$ & -0.000368 \\
\hline 124 & $\mathrm{H}$ & 0.000322 \\
\hline 125 & $\mathrm{H}$ & -0.005350 \\
\hline 126 & $\mathrm{H}$ & -0.002185 \\
\hline 127 & $\mathrm{H}$ & -0.000284 \\
\hline 128 & $\mathrm{H}$ & -0.000990 \\
\hline 129 & $\mathrm{H}$ & -0.001483 \\
\hline 130 & $\mathrm{H}$ & -0.002187 \\
\hline 131 & $\mathrm{H}$ & -0.005363 \\
\hline 132 & $\mathrm{H}$ & 0.000423 \\
\hline 133 & $\mathrm{H}$ & 0.000484 \\
\hline 134 & $\mathrm{H}$ & -0.000908 \\
\hline 135 & $\mathrm{H}$ & -0.001807 \\
\hline 136 & $\mathrm{H}$ & -0.000368 \\
\hline 137 & $\mathrm{H}$ & 0.000322 \\
\hline 138 & $\mathrm{H}$ & -0.000032 \\
\hline
\end{tabular}




$\begin{array}{ccc}139 & \mathrm{H} & 0.000012 \\ 140 & \mathrm{H} & 0.000180 \\ 141 & \mathrm{H} & -0.000007 \\ 142 & \mathrm{H} & 0.000009 \\ 143 & \mathrm{H} & -0.000005 \\ 144 & \mathrm{H} & 0.000019 \\ 145 & \mathrm{H} & -0.000006 \\ 146 & \mathrm{H} & 0.000143 \\ 147 & \mathrm{H} & -0.000011 \\ 148 & \mathrm{H} & -0.000012 \\ 149 & \mathrm{H} & 0.000015 \\ 150 & \mathrm{H} & 0.000004 \\ 151 & \mathrm{H} & 0.000119 \\ 152 & \mathrm{H} & -0.000016 \\ 153 & \mathrm{H} & -0.000020 \\ 154 & \mathrm{H} & 0.000179 \\ 155 & \mathrm{H} & -0.000015 \\ 156 & \mathrm{H} & -0.000015 \\ 157 & \mathrm{H} & 0.000179 \\ 158 & \mathrm{H} & -0.000020 \\ 159 & \mathrm{H} & 0.000004 \\ 160 & \mathrm{H} & -0.000016 \\ 161 & \mathrm{H} & 0.000120 \\ 162 & \mathrm{H} & 0.000015 \\ 163 & \mathrm{H} & -0.000012 \\ 164 & \mathrm{H} & -0.000011 \\ 165 & \mathrm{H} & 0.000010 \\ 166 & \mathrm{H} & -0.000004 \\ 167 & \mathrm{H} & -0.000010 \\ 168 & \mathrm{H} & 0.000012 \\ 169 & \mathrm{H} & -0.000029 \\ 170 & \mathrm{H} & 0.000187 \\ 171 & \mathrm{H} & -0.000007 \\ 172 & \mathrm{H} & 0.000142 \\ 173 & \mathrm{H} & 0.000019 \\ 174 & \mathrm{H} & -0.000306 \\ 175 & \mathrm{H} & 0.000239 \\ 176 & \mathrm{H} & 0.000136 \\ 177 & \mathrm{H} & -0.000222 \\ 178 & \mathrm{H} & -0.000486 \\ & & -0.000320 \\ 179 & -0.000195\end{array}$




$\begin{array}{ccc}183 & \mathrm{H} & 0.000137 \\ 184 & \mathrm{H} & 0.000229 \\ 185 & \mathrm{H} & -0.000328 \\ 186 & \mathrm{H} & -0.000224 \\ 187 & \mathrm{H} & 0.000212 \\ 188 & \mathrm{H} & 0.000320 \\ 189 & \mathrm{H} & -0.000486 \\ 190 & \mathrm{H} & 0.000004 \\ 191 & \mathrm{H} & -0.000009 \\ 192 & \mathrm{H} & 0.000004 \\ 193 & \mathrm{H} & -0.000002 \\ 194 & \mathrm{H} & 0.000098 \\ 195 & \mathrm{H} & -0.000008 \\ 196 & \mathrm{H} & -0.000006 \\ 197 & \mathrm{H} & -0.000012 \\ 198 & \mathrm{H} & 0.000039 \\ 199 & \mathrm{H} & 0.000005 \\ 200 & \mathrm{H} & 0.000006 \\ 201 & \mathrm{H} & -0.000002 \\ 202 & \mathrm{H} & -0.000000 \\ 203 & \mathrm{H} & -0.000008 \\ 204 & \mathrm{H} & 0.000087 \\ 205 & \mathrm{H} & -0.000005 \\ 206 & \mathrm{H} & -0.000013 \\ 207 & \mathrm{H} & 0.000084 \\ 208 & \mathrm{H} & 0.000006 \\ 209 & \mathrm{H} & -0.000002 \\ 210 & \mathrm{H} & 0.000005 \\ 211 & \mathrm{H} & -0.000005 \\ 212 & \mathrm{H} & -0.000013 \\ 213 & \mathrm{H} & 0.000084 \\ 214 & \mathrm{H} & -0.000008 \\ 215 & \mathrm{H} & 0.000087 \\ 216 & \mathrm{H} & -0.000000 \\ 217 & \mathrm{H} & -0.000009 \\ 218 & \mathrm{H} & 0.000067 \\ 219 & \mathrm{H} & -0.000003 \\ 220 & \mathrm{H} & 0.000006 \\ 221 & \mathrm{H} & -0.000000 \\ & \mathrm{H} & -0.000003 \\ 22 & -0.000004 \\ 22 & & -0.000081 \\ 22 & \end{array}$


$227 \quad \mathrm{H} \quad-0.000281$

$228 \mathrm{H} \quad-0.000998$

\section{OS $9 b^{2++}$}

Energy (with Zero Point Energy correction $)=-4562.591456(-4560.651693)$ Hartree Free energy $(298 \mathrm{~K})=-4560.800239$ Hartree

Zero number of imaginary frequency

21

$\mathrm{C}$

C

$\mathrm{C}$

C

C

$\mathrm{C}$

$\mathrm{C}$

$\mathrm{C}$

C

$\mathrm{C}$

$\mathrm{C}$

C

$\mathrm{C}$

C

$\mathrm{C}$

$\mathrm{C}$

C

C

C

C

C

C

C

C

C

C

C

C

C

C

C

C

$-11.77111600$

1.54191400

1.49499700

$-11.61417800$

0.30675300

0.85307100

$-10.31701500$

$-0.09393500$

0.42363100

$-9.22331800$

0.82054700

0.54789600

$-9.44156800$

2.03411800

1.25082600

$-10.68902600$

2.40483400

1.74822800

$-10.13110000$

$-1.37700900$

$-0.15896400$

$-8.82574900$

$-1.84823900$

$-0.47626600$

$-7.67283500$

$-0.98495500$

$-0.16122800$

$-7.91732100$

0.42175900

0.05169900

$-11.26544200$

$-2.20791400$

$-0.41830500$

$-11.08129200$

$-3.43338600$

$-1.07254700$

$-9.82161000$

$-3.86199800$

$-1.49447600$

$-8.71123900$

$-3.06052100$

$-1.16767100$

$-12.73124100$

$-0.56529300$

0.62222200

$-12.56466700$

$-1.76193300$

$-0.00555200$

$-6.34907500$

$-1.45288400$

$-0.03133800$

$-5.25981200$

$-0.51361600$

$-0.02669000$

$-5.52298900$

0.88117100

$-0.27702000$

$-6.85496100$

1.35795300

$-0.19091600$

$-3.92229300$

$-0.93578100$

0.16278000

$-2.86265800$

$-0.05861200$

$-0.03156100$

$-3.13053300$

1.29372500

$-0.45417400$

$-4.41891800$

1.74491000

$-0.57476500$

$-0.09158400$

3.54104500

$-1.14150100$

$-1.47140600$

3.23050200

$-1.05930100$

$-1.84233200$

1.96169300

$-0.65621500$

$-0.83695200$

1.00941500

$-0.33514800$

0.55149200

1.30461600

$-0.40987400$

0.89541000

2.61885300

$-0.82973800$

$-1.42264500$

$-0.23206200$

0.05210500

$-0.55854000$

$-1.30663100$

0.40749900

0.82989900

$-1.01140400$

0.33285600

1.41562600

0.22984400

$-0.05496400$

2.85559400

0.05616400

0.02842800

C

3.12339100

$-1.29561900$

0.45252100

C

4.41181500

$-1.74666500$

0.57431600 


\begin{tabular}{|c|c|c|c|}
\hline $\mathrm{C}$ & 5.51601200 & -0.88358200 & 0.27530200 \\
\hline $\mathrm{C}$ & 5.25288900 & 0.51072800 & 0.02248300 \\
\hline $\mathrm{C}$ & 3.91548700 & 0.93281300 & -0.16753300 \\
\hline $\mathrm{C}$ & 6.84788400 & -1.36089300 & 0.18937100 \\
\hline $\mathrm{C}$ & 7.91079600 & -0.42575100 & -0.05529200 \\
\hline $\mathrm{C}$ & 7.66627000 & 0.98138200 & 0.15479600 \\
\hline $\mathrm{C}$ & 6.34247300 & 1.44954200 & 0.02461900 \\
\hline $\mathrm{C}$ & 9.21692300 & -0.82553300 & -0.55112500 \\
\hline $\mathrm{C}$ & 10.30987900 & 0.09087200 & -0.43208000 \\
\hline $\mathrm{C}$ & 10.12403900 & 1.37522000 & 0.14766500 \\
\hline $\mathrm{C}$ & 8.81890700 & 1.84578300 & 0.46696100 \\
\hline $\mathrm{C}$ & 11.25794500 & 2.20812400 & 0.40248400 \\
\hline $\mathrm{C}$ & 11.07385100 & 3.43435200 & 1.05508200 \\
\hline $\mathrm{C}$ & 9.81464500 & 3.86210000 & 1.47973600 \\
\hline $\mathrm{C}$ & 8.70448400 & 3.05898000 & 1.15682700 \\
\hline $\mathrm{C}$ & 9.43604000 & -2.04178100 & -1.24885400 \\
\hline $\mathrm{C}$ & 10.68299800 & -2.41207400 & -1.74806400 \\
\hline $\mathrm{C}$ & 11.76393500 & -1.54555400 & -1.50247400 \\
\hline $\mathrm{C}$ & 11.60661800 & -0.30891000 & -0.86395900 \\
\hline $\mathrm{C}$ & 12.72308900 & 0.56558200 & -0.63869700 \\
\hline $\mathrm{C}$ & 12.55675300 & 1.76318900 & -0.01306800 \\
\hline $\mathrm{C}$ & -7.14741800 & 2.78822900 & -0.45823300 \\
\hline $\mathrm{C}$ & -10.92434900 & 3.70356400 & 2.53866000 \\
\hline $\mathrm{C}$ & -11.86601900 & 4.62158600 & 1.72134900 \\
\hline $\mathrm{C}$ & -9.61627200 & 4.46716900 & 2.81472600 \\
\hline $\mathrm{C}$ & -11.58225500 & 3.36939000 & 3.89957800 \\
\hline $\mathrm{C}$ & -9.68720200 & -5.18064100 & -2.27790800 \\
\hline $\mathrm{C}$ & -8.24497600 & -5.44409600 & -2.74981900 \\
\hline $\mathrm{C}$ & -10.59878400 & -5.13358600 & -3.52822000 \\
\hline $\mathrm{C}$ & -10.12442800 & -6.35267300 & -1.36550400 \\
\hline $\mathrm{C}$ & -6.04677500 & -2.89424400 & 0.19894600 \\
\hline $\mathrm{C}$ & 6.04065900 & 2.89041500 & -0.20900100 \\
\hline $\mathrm{C}$ & 7.13878200 & -2.79108600 & 0.45888100 \\
\hline $\mathrm{C}$ & 9.68080700 & 5.18158400 & 2.26180000 \\
\hline $\mathrm{C}$ & 10.11524900 & 6.35289300 & 1.34714400 \\
\hline $\mathrm{C}$ & 10.59500900 & 5.13669700 & 3.51028900 \\
\hline $\mathrm{C}$ & 8.23935200 & 5.44448700 & 2.73636500 \\
\hline $\mathrm{C}$ & 10.91894200 & -3.71169600 & -2.53684800 \\
\hline $\mathrm{C}$ & 9.61547500 & -4.49246300 & -2.78502700 \\
\hline $\mathrm{C}$ & 11.88706700 & -4.61618900 & -1.73560000 \\
\hline $\mathrm{C}$ & 11.54678400 & -3.37332600 & -3.91099500 \\
\hline $\mathrm{C}$ & -6.50368300 & 3.83710000 & 0.22836800 \\
\hline $\mathrm{C}$ & -6.77519400 & 5.16483100 & -0.08169700 \\
\hline $\mathrm{C}$ & -7.68093900 & 5.52129700 & -1.10005200 \\
\hline
\end{tabular}




\begin{tabular}{|c|c|c|c|}
\hline $\mathrm{C}$ & -8.31608400 & 4.46976200 & -1.77971300 \\
\hline $\mathrm{C}$ & -8.06746600 & 3.13651300 & -1.46244400 \\
\hline $\mathrm{C}$ & -5.19737100 & -3.62919100 & -0.64194300 \\
\hline $\mathrm{C}$ & -4.91387400 & -4.97231500 & -0.38286300 \\
\hline $\mathrm{C}$ & -5.44973400 & -5.63591900 & 0.73053400 \\
\hline $\mathrm{C}$ & -6.29297100 & -4.88542200 & 1.57210000 \\
\hline $\mathrm{C}$ & -6.59201800 & -3.55305000 & 1.31470400 \\
\hline $\mathrm{C}$ & 8.05966000 & -3.13891200 & 1.46731700 \\
\hline $\mathrm{C}$ & 8.30517400 & -4.46887300 & 1.78071600 \\
\hline $\mathrm{C}$ & 7.67129800 & -5.52483000 & 1.09717100 \\
\hline $\mathrm{C}$ & 6.76913200 & -5.17105400 & 0.08112400 \\
\hline $\mathrm{C}$ & 6.49842500 & -3.83789300 & -0.22667700 \\
\hline $\mathrm{C}$ & 6.58626900 & 3.54649800 & -1.32620900 \\
\hline $\mathrm{C}$ & 6.28737100 & 4.87823900 & -1.58692500 \\
\hline $\mathrm{C}$ & 5.44405300 & 5.63088000 & -0.74734700 \\
\hline $\mathrm{C}$ & 4.90792900 & 4.97005300 & 0.36758300 \\
\hline $\mathrm{C}$ & 5.19115400 & 3.62751700 & 0.62993400 \\
\hline $\mathrm{C}$ & -7.93368800 & 7.00217400 & -1.42503200 \\
\hline $\mathrm{C}$ & -8.95776400 & 7.18585900 & -2.56089000 \\
\hline $\mathrm{C}$ & -6.60105700 & 7.66000800 & -1.86016300 \\
\hline $\mathrm{C}$ & -8.47207600 & 7.72067000 & -0.16380100 \\
\hline $\mathrm{C}$ & -5.15175100 & -7.10985300 & 1.05309400 \\
\hline $\mathrm{C}$ & -4.22623100 & -7.76189100 & 0.00868600 \\
\hline $\mathrm{C}$ & -6.47846800 & -7.90644500 & 1.08968000 \\
\hline $\mathrm{C}$ & -4.46336100 & -7.19933100 & 2.43681800 \\
\hline $\mathrm{C}$ & 5.14626100 & 7.10403400 & -1.07357100 \\
\hline $\mathrm{C}$ & 4.22060800 & 7.75871200 & -0.03093300 \\
\hline $\mathrm{C}$ & 4.45811700 & 7.19016100 & -2.45763500 \\
\hline $\mathrm{C}$ & 6.47305400 & 7.90042100 & -1.11187900 \\
\hline $\mathrm{C}$ & 7.97586700 & -6.98245300 & 1.47729300 \\
\hline $\mathrm{C}$ & 9.49069300 & -7.25269500 & 1.30652800 \\
\hline $\mathrm{C}$ & 7.20339400 & -7.98922500 & 0.60422800 \\
\hline $\mathrm{C}$ & 7.57772400 & -7.21318000 & 2.95602000 \\
\hline $\mathrm{C}$ & -0.90251000 & -2.62059300 & 0.82816300 \\
\hline $\mathrm{C}$ & 0.08445000 & -3.54258700 & 1.14071400 \\
\hline $\mathrm{C}$ & 1.46423100 & -3.23202800 & 1.05859000 \\
\hline $\mathrm{C}$ & 1.83524300 & -1.96341600 & 0.65480600 \\
\hline $\mathrm{H}$ & -12.76845700 & 1.81779500 & 1.82525800 \\
\hline $\mathrm{H}$ & -8.59758100 & 2.67864600 & 1.43617500 \\
\hline $\mathrm{H}$ & -11.95850800 & -4.04344300 & -1.26655500 \\
\hline $\mathrm{H}$ & -7.73221000 & -3.38634300 & -1.48215200 \\
\hline $\mathrm{H}$ & -13.71663600 & -0.24241200 & 0.94605400 \\
\hline $\mathrm{H}$ & -13.41736200 & -2.40755700 & -0.19638800 \\
\hline $\mathrm{H}$ & -3.74281700 & -1.96407000 & 0.44205700 \\
\hline
\end{tabular}




\begin{tabular}{|c|c|c|c|}
\hline $\mathrm{H}$ & -4.61504900 & 2.76298600 & -0.88768000 \\
\hline $\mathrm{H}$ & 0.20119100 & 4.53644700 & -1.46173600 \\
\hline $\mathrm{H}$ & -2.20860300 & 3.98623500 & -1.31485600 \\
\hline $\mathrm{H}$ & 1.93440100 & 2.91586900 & -0.91209800 \\
\hline $\mathrm{H}$ & 4.60796500 & -2.76417400 & 0.88897100 \\
\hline $\mathrm{H}$ & 3.73618600 & 1.96052700 & -0.44902200 \\
\hline $\mathrm{H}$ & 11.95076400 & 4.04588100 & 1.24582400 \\
\hline $\mathrm{H}$ & 7.72577500 & 3.38412200 & 1.47302500 \\
\hline $\mathrm{H}$ & 8.59311800 & -2.68911900 & -1.42889800 \\
\hline $\mathrm{H}$ & 12.76058100 & -1.82019100 & -1.83589900 \\
\hline $\mathrm{H}$ & 13.70793900 & 0.24328400 & -0.96476700 \\
\hline $\mathrm{H}$ & 13.40902800 & 2.41047300 & 0.17398000 \\
\hline $\mathrm{H}$ & -11.41860200 & 4.88418400 & 0.75588500 \\
\hline $\mathrm{H}$ & -12.83413700 & 4.14836800 & 1.52534800 \\
\hline $\mathrm{H}$ & -12.05776900 & 5.54913500 & 2.27249600 \\
\hline $\mathrm{H}$ & -9.12014000 & 4.78495100 & 1.89135200 \\
\hline $\mathrm{H}$ & -8.90918400 & 3.86641000 & 3.39900800 \\
\hline $\mathrm{H}$ & -9.83714300 & 5.36867800 & 3.39556200 \\
\hline $\mathrm{H}$ & -12.55111600 & 2.87305700 & 3.78350600 \\
\hline $\mathrm{H}$ & -10.93899500 & 2.71651300 & 4.50016200 \\
\hline $\mathrm{H}$ & -11.75320400 & 4.29073500 & 4.46716600 \\
\hline $\mathrm{H}$ & -7.54716500 & -5.54501400 & -1.91146800 \\
\hline $\mathrm{H}$ & -8.21418800 & -6.37985400 & -3.31786900 \\
\hline $\mathrm{H}$ & -7.87943500 & -4.64860700 & -3.40989700 \\
\hline $\mathrm{H}$ & -10.31538200 & -4.30990200 & -4.19307700 \\
\hline $\mathrm{H}$ & -10.50947900 & -6.06920100 & -4.09150400 \\
\hline $\mathrm{H}$ & -11.65422300 & -5.00701100 & -3.26737800 \\
\hline $\mathrm{H}$ & -11.16124500 & -6.24584300 & -1.02992300 \\
\hline $\mathrm{H}$ & -10.04814900 & -7.30128600 & -1.90920200 \\
\hline $\mathrm{H}$ & -9.48799300 & -6.41752900 & -0.47556100 \\
\hline $\mathrm{H}$ & 9.47697100 & 6.41616500 & 0.45840800 \\
\hline $\mathrm{H}$ & 10.03928600 & 7.30208300 & 1.88988100 \\
\hline $\mathrm{H}$ & 11.15147300 & 6.24653900 & 1.00959900 \\
\hline $\mathrm{H}$ & 10.31351100 & 4.31365900 & 4.17675400 \\
\hline $\mathrm{H}$ & 11.64997800 & 5.01043800 & 3.24739700 \\
\hline $\mathrm{H}$ & 10.50627500 & 6.07296100 & 4.07259100 \\
\hline $\mathrm{H}$ & 7.87583800 & 4.64955500 & 3.39823000 \\
\hline $\mathrm{H}$ & 8.20896600 & 6.38095700 & 3.30326600 \\
\hline $\mathrm{H}$ & 7.53973100 & 5.54373700 & 1.89931900 \\
\hline $\mathrm{H}$ & 8.89143200 & -3.90517400 & -3.36215300 \\
\hline $\mathrm{H}$ & 9.83726400 & -5.39590100 & -3.36257700 \\
\hline $\mathrm{H}$ & 9.13865600 & -4.80777300 & -1.85059200 \\
\hline $\mathrm{H}$ & 12.85332600 & -4.13164800 & -1.55942600 \\
\hline $\mathrm{H}$ & 11.46173200 & -4.88286100 & -0.76143700 \\
\hline
\end{tabular}




\begin{tabular}{|c|c|c|c|}
\hline $\mathrm{H}$ & 12.07931600 & -5.54239200 & -2.28883800 \\
\hline $\mathrm{H}$ & 10.88351300 & -2.73141300 & -4.50156800 \\
\hline $\mathrm{H}$ & 11.72029000 & -4.29407800 & -4.47883400 \\
\hline $\mathrm{H}$ & 12.51032500 & -2.86270100 & -3.81373100 \\
\hline $\mathrm{H}$ & -5.81441700 & 3.60747700 & 1.03639800 \\
\hline $\mathrm{H}$ & -6.27173700 & 5.94080700 & 0.48703600 \\
\hline $\mathrm{H}$ & -9.01826100 & 4.68369300 & -2.57697000 \\
\hline $\mathrm{H}$ & -8.57538900 & 2.35357900 & -2.01761100 \\
\hline $\mathrm{H}$ & -4.77566500 & -3.15834700 & -1.52631400 \\
\hline $\mathrm{H}$ & -4.26729600 & -5.50137000 & -1.07378600 \\
\hline $\mathrm{H}$ & -6.72689900 & -5.34949200 & 2.45265500 \\
\hline $\mathrm{H}$ & -7.24784800 & -3.01031900 & 1.98919500 \\
\hline $\mathrm{H}$ & 8.56602100 & -2.35533000 & 2.02294600 \\
\hline $\mathrm{H}$ & 9.00615300 & -4.68878400 & 2.57999600 \\
\hline $\mathrm{H}$ & 6.26579500 & -5.94172600 & -0.49129300 \\
\hline $\mathrm{H}$ & 5.81035500 & -3.60884300 & -1.03594200 \\
\hline $\mathrm{H}$ & 7.24218200 & 3.00206900 & -1.99923900 \\
\hline $\mathrm{H}$ & 6.72149700 & 5.34010700 & -2.46853700 \\
\hline $\mathrm{H}$ & 4.26136900 & 5.50089400 & 1.05715500 \\
\hline $\mathrm{H}$ & 4.76930300 & 3.15889900 & 1.51541700 \\
\hline $\mathrm{H}$ & -8.61945600 & 6.72826000 & -3.49742000 \\
\hline $\mathrm{H}$ & -9.10321800 & 8.25376100 & -2.75304100 \\
\hline $\mathrm{H}$ & -9.93597700 & 6.76391600 & -2.30383100 \\
\hline $\mathrm{H}$ & -6.19720400 & 7.17715300 & -2.75726600 \\
\hline $\mathrm{H}$ & -6.76382500 & 8.71875600 & -2.09066200 \\
\hline $\mathrm{H}$ & -5.84012600 & 7.60574800 & -1.07415200 \\
\hline $\mathrm{H}$ & -9.42046200 & 7.28154300 & 0.16595700 \\
\hline $\mathrm{H}$ & -7.76746100 & 7.66979700 & 0.67293000 \\
\hline $\mathrm{H}$ & -8.64923900 & 8.77946300 & -0.38305200 \\
\hline $\mathrm{H}$ & -4.66931500 & -7.75502700 & -0.99359800 \\
\hline $\mathrm{H}$ & -3.25046500 & -7.26461600 & -0.04284100 \\
\hline $\mathrm{H}$ & -4.04650100 & -8.80736000 & 0.27973800 \\
\hline $\mathrm{H}$ & -6.98859800 & -7.86759200 & 0.12053800 \\
\hline $\mathrm{H}$ & -7.16751000 & -7.52287300 & 1.84924300 \\
\hline $\mathrm{H}$ & -6.27991400 & -8.95818100 & 1.32477200 \\
\hline $\mathrm{H}$ & -3.51393000 & -6.65107700 & 2.44140600 \\
\hline $\mathrm{H}$ & -5.09084900 & -6.79038400 & 3.23542600 \\
\hline $\mathrm{H}$ & -4.25096300 & -8.24534900 & 2.68498700 \\
\hline $\mathrm{H}$ & 3.24480200 & 7.26161900 & 0.02165000 \\
\hline $\mathrm{H}$ & 4.04098400 & 8.80351600 & -0.30460700 \\
\hline $\mathrm{H}$ & 4.66351800 & 7.75430300 & 0.97144200 \\
\hline $\mathrm{H}$ & 3.50865000 & 6.64196400 & -2.46105600 \\
\hline $\mathrm{H}$ & 5.08572000 & 6.77921600 & -3.25512500 \\
\hline $\mathrm{H}$ & 4.24584300 & 8.23558400 & -2.70840300 \\
\hline
\end{tabular}




$\begin{array}{lrrr}\mathrm{H} & 7.16219200 & 7.51492300 & -1.87037800 \\ \mathrm{H} & 6.27462500 & 8.95159000 & -1.34959700 \\ \mathrm{H} & 6.98301400 & 7.86390700 & -0.14255800 \\ \mathrm{H} & 10.09982900 & -6.59972200 & 1.94015200 \\ \mathrm{H} & 9.72004900 & -8.28786900 & 1.58276700 \\ \mathrm{H} & 9.80379300 & -7.10382700 & 0.26677800 \\ \mathrm{H} & 7.46066500 & -7.89504500 & -0.45700100 \\ \mathrm{H} & 7.45516300 & -9.00915300 & 0.91214300 \\ \mathrm{H} & 6.11817700 & -7.87525500 & 0.70831500 \\ \mathrm{H} & 6.50709400 & -7.03595900 & 3.10922200 \\ \mathrm{H} & 7.79403700 & -8.24768700 & 3.24510500 \\ \mathrm{H} & 8.12862700 & -6.55669100 & 3.63744000 \\ \mathrm{H} & -1.94151600 & -2.91746300 & 0.91079900 \\ \mathrm{H} & -0.20837700 & -4.53776900 & 1.46158000 \\ \mathrm{H} & 2.20143200 & -3.98754400 & 1.31475400\end{array}$

Mulliken spin densities:
$\begin{array}{lll}1 & \text { C } & -0.139298\end{array}$
$\begin{array}{lll}2 & \mathrm{C} & 0.041888\end{array}$
3 C -0.019518
$\begin{array}{lll}4 & \mathrm{C} & -0.073787\end{array}$
5 C $\quad-0.040870$
$\begin{array}{lll}6 & \mathrm{C} & 0.018805\end{array}$
$\begin{array}{lll}7 & \mathrm{C} & -0.025979\end{array}$
8 C -0.015021
$\begin{array}{lll}9 & \mathrm{C} & 0.034363\end{array}$
$10 \mathrm{C} \quad-0.026618$
11 C -0.026239
12 C 0.026073
13 C -0.032697
14 C 0.005839
15 C -0.041033
16 C 0.016288
17 C -0.089002
18 C -0.012677
19 C 0.062620
20 C -0.165656
21 C -0.006873
22 C -0.066282
23 C 0.038323
$24 \mathrm{C} \quad-0.093065$
$25 \mathrm{C} \quad 0.049541$
26 C -0.055361
27 C 0.034336
28 C -0.043219 


$$
\begin{aligned}
& 29 \mathrm{C} \quad 0.057730 \\
& 30 \text { C }-0.055309 \\
& 31 \text { C } 0.114080 \\
& 32 \text { C }-0.057884 \\
& 33 \text { C } 0.042875 \\
& 34 \text { C }-0.112965 \\
& 35 \mathrm{C} \quad 0.065525 \\
& 36 \mathrm{C} \quad-0.038105 \\
& 37 \text { C } 0.093750 \\
& 38 \text { C }-0.062634 \\
& 39 \mathrm{C} \quad 0.011623 \\
& 40 \text { C } 0.009290 \\
& 41 \text { C } 0.166243 \\
& 42 \mathrm{C} \quad 0.025821 \\
& 43 \text { C }-0.034872 \\
& 44 \text { C } 0.091359 \\
& 45 \text { C } 0.073178 \\
& 46 \text { C } \quad 0.020198 \\
& 47 \text { C } 0.025759 \\
& 48 \mathrm{C} \quad 0.016140 \\
& 49 \text { C } 0.025905 \\
& 50 \quad \mathrm{C}-0.026028 \\
& 51 \mathrm{C} \quad 0.033200 \\
& 52 \text { C }-0.006980 \\
& 53 \text { C } 0.038462 \\
& 54 \text { C }-0.017136 \\
& 55 \text { C } 0.136527 \\
& 56 \text { C }-0.041334 \\
& 57 \text { C } 0.040114 \\
& 58 \text { C }-0.016530 \\
& 59 \mathrm{C} \quad 0.002288 \\
& \begin{array}{lll}
60 & \mathrm{C} & -0.000780
\end{array} \\
& 61 \mathrm{C}-0.000791 \\
& \begin{array}{lll}
62 & \mathrm{C} & 0.000051
\end{array} \\
& 63 \text { C }-0.000409 \\
& 64 \mathrm{C} \quad 0.001856 \\
& \begin{array}{lll}
65 & \mathrm{C} & -0.000084
\end{array} \\
& 66 \text { C }-0.001119 \\
& 67 \text { C }-0.001375 \\
& \begin{array}{lll}
68 & \mathrm{C} & 0.006279
\end{array} \\
& 69 \text { C }-0.006411 \\
& 70 \text { C }-0.002750 \\
& 71 \text { C }-0.001883 \\
& 72 \text { C } 0.001392
\end{aligned}
$$




\begin{tabular}{|c|c|c|}
\hline 73 & $\mathrm{C}$ & 0.001133 \\
\hline 74 & $\mathrm{C}$ & 0.000081 \\
\hline 75 & $\mathrm{C}$ & 0.000694 \\
\hline 76 & $\mathrm{C}$ & -0.000036 \\
\hline 77 & $\mathrm{C}$ & 0.000798 \\
\hline 78 & $\mathrm{C}$ & 0.000475 \\
\hline 79 & $\mathrm{C}$ & -0.019307 \\
\hline 80 & $\mathrm{C}$ & 0.008602 \\
\hline 81 & $\mathrm{C}$ & -0.036285 \\
\hline 82 & $\mathrm{C}$ & 0.014352 \\
\hline 83 & $\mathrm{C}$ & -0.028077 \\
\hline 84 & $\mathrm{C}$ & -0.007591 \\
\hline 85 & $\mathrm{C}$ & 0.003088 \\
\hline 86 & $\mathrm{C}$ & -0.008015 \\
\hline 87 & $\mathrm{C}$ & 0.001798 \\
\hline 88 & $\mathrm{C}$ & -0.005138 \\
\hline 89 & $\mathrm{C}$ & 0.024479 \\
\hline 90 & $\mathrm{C}$ & -0.013637 \\
\hline 91 & $\mathrm{C}$ & 0.035808 \\
\hline 92 & $\mathrm{C}$ & -0.009323 \\
\hline 93 & $\mathrm{C}$ & 0.022257 \\
\hline 94 & $\mathrm{C}$ & 0.005356 \\
\hline 95 & $\mathrm{C}$ & -0.001911 \\
\hline 96 & $\mathrm{C}$ & 0.008336 \\
\hline 97 & $\mathrm{C}$ & -0.003212 \\
\hline 98 & $\mathrm{C}$ & 0.007839 \\
\hline 99 & $\mathrm{C}$ & 0.002105 \\
\hline 100 & $\mathrm{C}$ & -0.000044 \\
\hline 101 & $\mathrm{C}$ & -0.001431 \\
\hline 102 & $\mathrm{C}$ & -0.001356 \\
\hline 103 & $\mathrm{C}$ & 0.000470 \\
\hline 104 & $\mathrm{C}$ & -0.000011 \\
\hline 105 & $\mathrm{C}$ & -0.000300 \\
\hline 106 & $\mathrm{C}$ & -0.000312 \\
\hline 107 & $\mathrm{C}$ & -0.000489 \\
\hline 108 & $\mathrm{C}$ & 0.000011 \\
\hline 109 & $\mathrm{C}$ & 0.000325 \\
\hline 110 & $\mathrm{C}$ & 0.000310 \\
\hline 111 & $\mathrm{C}$ & -0.002076 \\
\hline 112 & $\mathrm{C}$ & 0.001278 \\
\hline 113 & $\mathrm{C}$ & 0.000071 \\
\hline 114 & $\mathrm{C}$ & 0.001472 \\
\hline 115 & $\mathrm{C}$ & 0.056104 \\
\hline 116 & $\mathrm{C}$ & -0.049644 \\
\hline
\end{tabular}




$\begin{array}{ccc}117 & \mathrm{C} & 0.055933 \\ 118 & \mathrm{C} & -0.034213 \\ 119 & \mathrm{H} & 0.005893 \\ 120 & \mathrm{H} & 0.002306 \\ 121 & \mathrm{H} & -0.000957 \\ 122 & \mathrm{H} & -0.000106 \\ 123 & \mathrm{H} & 0.001678 \\ 124 & \mathrm{H} & -0.000562 \\ 125 & \mathrm{H} & 0.000293 \\ 126 & \mathrm{H} & 0.003371 \\ 127 & \mathrm{H} & -0.002057 \\ 128 & \mathrm{H} & 0.002321 \\ 129 & \mathrm{H} & 0.002296 \\ 130 & \mathrm{H} & -0.003397 \\ 131 & \mathrm{H} & -0.000387 \\ 132 & \mathrm{H} & 0.000958 \\ 133 & \mathrm{H} & 0.000148 \\ 134 & \mathrm{H} & -0.002216 \\ 135 & \mathrm{H} & -0.005774 \\ 136 & \mathrm{H} & -0.001641 \\ 137 & \mathrm{H} & 0.000574 \\ 138 & \mathrm{H} & 0.000094 \\ 139 & \mathrm{H} & -0.000020 \\ 140 & \mathrm{H} & -0.000697 \\ 141 & \mathrm{H} & 0.000045 \\ 142 & \mathrm{H} & -0.000054 \\ 143 & \mathrm{H} & 0.000027 \\ 144 & \mathrm{H} & -0.000040 \\ 145 & \mathrm{H} & 0.000020 \\ 146 & \mathrm{H} & -0.000588 \\ 157 & \mathrm{H} & 0.000011 \\ 159 & \mathrm{H} & -0.000063 \\ 148 & \mathrm{H} & 0.000039 \\ 149 & \mathrm{H} & -0.000037 \\ 150 & \mathrm{H} & -0.000005 \\ 151 & \mathrm{H} & -0.000237 \\ 152 & \mathrm{H} & 0.000056 \\ 153 & \mathrm{H} & 0.000062 \\ 154 & \mathrm{H} & -0.000342 \\ & \mathrm{H} & 0.000025 \\ 155 & -0.000026 \\ 157 & 0.0000056\end{array}$




$\begin{array}{ccc}161 & \mathrm{H} & 0.000246 \\ 162 & \mathrm{H} & 0.000038 \\ 163 & \mathrm{H} & -0.000039 \\ 164 & \mathrm{H} & -0.000012 \\ 165 & \mathrm{H} & 0.000054 \\ 166 & \mathrm{H} & -0.000026 \\ 167 & \mathrm{H} & -0.000053 \\ 168 & \mathrm{H} & 0.000020 \\ 169 & \mathrm{H} & -0.000082 \\ 170 & \mathrm{H} & 0.000717 \\ 171 & \mathrm{H} & -0.000021 \\ 172 & \mathrm{H} & 0.000597 \\ 173 & \mathrm{H} & 0.000035 \\ 174 & \mathrm{H} & 0.000985 \\ 175 & \mathrm{H} & -0.000597 \\ 176 & \mathrm{H} & -0.000690 \\ 177 & \mathrm{H} & 0.001262 \\ 178 & \mathrm{H} & 0.000387 \\ 179 & \mathrm{H} & -0.000304 \\ 180 & \mathrm{H} & -0.000206 \\ 181 & \mathrm{H} & 0.000180 \\ 182 & \mathrm{H} & -0.001099 \\ 183 & \mathrm{H} & 0.000694 \\ 184 & \mathrm{H} & 0.000593 \\ 185 & \mathrm{H} & -0.001112 \\ 186 & \mathrm{H} & -0.000190 \\ 187 & \mathrm{H} & 0.000214 \\ 188 & \mathrm{H} & 0.000312 \\ 189 & \mathrm{H} & -0.000399 \\ 190 & \mathrm{H} & -0.000032 \\ 191 & \mathrm{H} & 0.000040 \\ 192 & \mathrm{H} & -0.000023 \\ 193 & \mathrm{H} & 0.000009 \\ 194 & \mathrm{H} & -0.000552 \\ 195 & \mathrm{H} & 0.000048 \\ 196 & \mathrm{H} & 0.000031 \\ 197 & \mathrm{H} & 0.000061 \\ 198 & \mathrm{H} & -0.000342 \\ 199 & \mathrm{H} & -0.000008 \\ 200 & \mathrm{H} & -0.000006 \\ 204 & \mathrm{H} & 0.000005 \\ & \mathrm{H} & 0.000001 \\ & & -0.000109\end{array}$




$$
\begin{array}{ccc}
205 & \mathrm{H} & 0.000003 \\
206 & \mathrm{H} & 0.000014 \\
207 & \mathrm{H} & -0.000084 \\
208 & \mathrm{H} & 0.000006 \\
209 & \mathrm{H} & -0.000005 \\
210 & \mathrm{H} & 0.000009 \\
211 & \mathrm{H} & -0.000003 \\
212 & \mathrm{H} & -0.000015 \\
213 & \mathrm{H} & 0.000088 \\
214 & \mathrm{H} & -0.000012 \\
215 & \mathrm{H} & 0.000112 \\
216 & \mathrm{H} & -0.000001 \\
217 & \mathrm{H} & -0.000053 \\
218 & \mathrm{H} & 0.000373 \\
219 & \mathrm{H} & -0.000017 \\
220 & \mathrm{H} & 0.000037 \\
221 & \mathrm{H} & -0.000030 \\
222 & \mathrm{H} & 0.000020 \\
223 & \mathrm{H} & -0.000016 \\
224 & \mathrm{H} & 0.000394 \\
225 & \mathrm{H} & -0.000064 \\
226 & \mathrm{H} & -0.002330 \\
227 & \mathrm{H} & 0.002056 \\
228 & \mathrm{H} & -0.002349
\end{array}
$$

\section{CS 9 $\mathbf{b}^{2+}$}

Energy (with Zero Point Energy correction $)=-4562.593816(-4560.655035)$ Hartree Free energy $(298 \mathrm{~K})=-4560.803696$ Hartree

Zero number of imaginary frequency

\section{1}

$\begin{array}{lrrr}\mathrm{C} & -11.76064000 & 1.54185000 & 1.49994100 \\ \mathrm{C} & -11.60405400 & 0.30812800 & 0.85715300 \\ \mathrm{C} & -10.30679400 & -0.09096500 & 0.42457800 \\ \mathrm{C} & -9.21328000 & 0.82348400 & 0.54971500 \\ \mathrm{C} & -9.43196000 & 2.03655900 & 1.24882100 \\ \mathrm{C} & -10.67999800 & 2.40554200 & 1.74948500 \\ \mathrm{C} & -10.12248000 & -1.37352700 & -0.16073100 \\ \mathrm{C} & -8.81766200 & -1.84405700 & -0.48122200 \\ \mathrm{C} & -7.66564600 & -0.98169500 & -0.16508800 \\ \mathrm{C} & -7.90687600 & 0.42394800 & 0.05452200 \\ \mathrm{C} & -11.25595500 & -2.20421200 & -0.41938600 \\ \mathrm{C} & -11.07156400 & -3.42763300 & -1.07619100 \\ \mathrm{C} & -9.81189800 & -3.85567100 & -1.50180900 \\ \mathrm{C} & -8.70209700 & -3.05545500 & -1.17537100 \\ \mathrm{C} & -12.72143700 & -0.56549600 & 0.62686600\end{array}$




\begin{tabular}{|c|c|c|c|}
\hline $\mathrm{C}$ & -12.55598800 & -1.75971500 & -0.00310400 \\
\hline $\mathrm{C}$ & -6.34112300 & -1.45309700 & -0.03666100 \\
\hline $\mathrm{C}$ & -5.25302900 & -0.51425500 & -0.03546600 \\
\hline $\mathrm{C}$ & -5.51807900 & 0.88222300 & -0.28773000 \\
\hline $\mathrm{C}$ & -6.84216400 & 1.35957100 & -0.19460500 \\
\hline $\mathrm{C}$ & -3.91882500 & -0.93269400 & 0.16243000 \\
\hline $\mathrm{C}$ & -2.85511700 & -0.05466200 & -0.03449100 \\
\hline $\mathrm{C}$ & -3.12479200 & 1.29743500 & -0.46859400 \\
\hline $\mathrm{C}$ & -4.40997000 & 1.74601100 & -0.59240900 \\
\hline $\mathrm{C}$ & -0.08521500 & 3.53634300 & -1.16742500 \\
\hline $\mathrm{C}$ & -1.46152100 & 3.23018200 & -1.08535100 \\
\hline $\mathrm{C}$ & -1.83601000 & 1.96154500 & -0.67335000 \\
\hline $\mathrm{C}$ & -0.83351200 & 1.01273200 & -0.34444700 \\
\hline $\mathrm{C}$ & 0.55330500 & 1.30470900 & -0.41969200 \\
\hline $\mathrm{C}$ & 0.90126900 & 2.61102100 & -0.84712600 \\
\hline $\mathrm{C}$ & -1.42677700 & -0.22945600 & 0.05199100 \\
\hline $\mathrm{C}$ & -0.56063600 & -1.30757100 & 0.41636400 \\
\hline $\mathrm{C}$ & 0.82618800 & -1.01556200 & 0.34120800 \\
\hline $\mathrm{C}$ & 1.41945700 & 0.22638900 & -0.05585800 \\
\hline $\mathrm{C}$ & 2.84780300 & 0.05143400 & 0.03038000 \\
\hline $\mathrm{C}$ & 3.11744300 & -1.30009400 & 0.46614100 \\
\hline $\mathrm{C}$ & 4.40262500 & -1.74835500 & 0.59140200 \\
\hline $\mathrm{C}$ & 5.51086500 & -0.88511400 & 0.28550100 \\
\hline $\mathrm{C}$ & 5.24577900 & 0.51086000 & 0.03057000 \\
\hline $\mathrm{C}$ & 3.91159800 & 0.92901400 & -0.16806700 \\
\hline $\mathrm{C}$ & 6.83495700 & -1.36270800 & 0.19301000 \\
\hline $\mathrm{C}$ & 7.90004300 & -0.42782100 & -0.05785400 \\
\hline $\mathrm{C}$ & 7.65847700 & 0.97832200 & 0.15849000 \\
\hline $\mathrm{C}$ & 6.33392800 & 1.44953400 & 0.02945500 \\
\hline $\mathrm{C}$ & 9.20663100 & -0.82816200 & -0.55245800 \\
\hline $\mathrm{C}$ & 10.29929300 & 0.08824000 & -0.43242300 \\
\hline $\mathrm{C}$ & 10.11480400 & 1.37220400 & 0.14974200 \\
\hline $\mathrm{C}$ & 8.81017000 & 1.84204100 & 0.47172400 \\
\hline $\mathrm{C}$ & 11.24777000 & 2.20495100 & 0.40405100 \\
\hline $\mathrm{C}$ & 11.06327800 & 3.42940700 & 1.05884900 \\
\hline $\mathrm{C}$ & 9.80403000 & 3.85663600 & 1.48661100 \\
\hline $\mathrm{C}$ & 8.69452800 & 3.05454300 & 1.16393500 \\
\hline $\mathrm{C}$ & 9.42641800 & -2.04405600 & -1.24640300 \\
\hline $\mathrm{C}$ & 10.67409100 & -2.41281000 & -1.74826700 \\
\hline $\mathrm{C}$ & 11.75346200 & -1.54554500 & -1.50591300 \\
\hline $\mathrm{C}$ & 11.59621700 & -0.31007300 & -0.86683900 \\
\hline $\mathrm{C}$ & 12.71288000 & 0.56593500 & -0.64194300 \\
\hline $\mathrm{C}$ & 12.54741200 & 1.76140000 & -0.01445500 \\
\hline $\mathrm{C}$ & -7.13069600 & 2.79354400 & -0.45989600 \\
\hline
\end{tabular}




\begin{tabular}{|c|c|c|c|}
\hline $\mathrm{C}$ & -10.91491900 & 3.70597100 & 2.53779500 \\
\hline $\mathrm{C}$ & -11.85776300 & 4.62233700 & 1.72007700 \\
\hline $\mathrm{C}$ & -9.60697700 & 4.47094300 & 2.81097300 \\
\hline $\mathrm{C}$ & -11.57094300 & 3.37456600 & 3.90019900 \\
\hline $\mathrm{C}$ & -9.67969500 & -5.17196900 & -2.28950700 \\
\hline $\mathrm{C}$ & -8.23726900 & -5.43769700 & -2.75953100 \\
\hline $\mathrm{C}$ & -10.58870800 & -5.11789400 & -3.54138900 \\
\hline $\mathrm{C}$ & -10.12167700 & -6.34642400 & -1.38258200 \\
\hline $\mathrm{C}$ & -6.04103300 & -2.89303000 & 0.19676400 \\
\hline $\mathrm{C}$ & 6.03383500 & 2.88894600 & -0.20726200 \\
\hline $\mathrm{C}$ & 7.12271200 & -2.79632200 & 0.46055000 \\
\hline $\mathrm{C}$ & 9.67201200 & 5.17404900 & 2.27245200 \\
\hline $\mathrm{C}$ & 10.11110600 & 6.34754200 & 1.36289300 \\
\hline $\mathrm{C}$ & 10.58347400 & 5.12278900 & 3.52267900 \\
\hline $\mathrm{C}$ & 8.23022100 & 5.43908000 & 2.74481800 \\
\hline $\mathrm{C}$ & 10.90995000 & -3.71431600 & -2.53453700 \\
\hline $\mathrm{C}$ & 9.60660000 & -4.49591800 & -2.78099700 \\
\hline $\mathrm{C}$ & 11.87803900 & -4.61733500 & -1.73167900 \\
\hline $\mathrm{C}$ & 11.53744300 & -3.37931500 & -3.90956000 \\
\hline $\mathrm{C}$ & -6.49199700 & 3.83634900 & 0.23866700 \\
\hline $\mathrm{C}$ & -6.75459200 & 5.16714500 & -0.06909600 \\
\hline $\mathrm{C}$ & -7.64812100 & 5.53082400 & -1.09459400 \\
\hline $\mathrm{C}$ & -8.27866400 & 4.48411200 & -1.78597900 \\
\hline $\mathrm{C}$ & -8.03670400 & 3.14834600 & -1.47302100 \\
\hline $\mathrm{C}$ & -5.17923400 & -3.62682700 & -0.63339900 \\
\hline $\mathrm{C}$ & -4.89820500 & -4.96995800 & -0.37198700 \\
\hline $\mathrm{C}$ & -5.44792700 & -5.63480000 & 0.73379500 \\
\hline $\mathrm{C}$ & -6.30323400 & -4.88555000 & 1.56498900 \\
\hline $\mathrm{C}$ & -6.60126800 & -3.55403100 & 1.30441000 \\
\hline $\mathrm{C}$ & 8.03078800 & -3.14976700 & 1.47723500 \\
\hline $\mathrm{C}$ & 8.27104400 & -4.48192000 & 1.78648000 \\
\hline $\mathrm{C}$ & 7.64201400 & -5.53370200 & 1.09206900 \\
\hline $\mathrm{C}$ & 6.75053900 & -5.17369400 & 0.06975300 \\
\hline $\mathrm{C}$ & 6.48740200 & -3.83778000 & -0.23587400 \\
\hline $\mathrm{C}$ & 6.59354900 & 3.54724300 & -1.31677700 \\
\hline $\mathrm{C}$ & 6.29523000 & 4.87806100 & -1.58059100 \\
\hline $\mathrm{C}$ & 5.44015700 & 5.62929200 & -0.75095200 \\
\hline $\mathrm{C}$ & 4.89099400 & 4.96717000 & 0.35674300 \\
\hline $\mathrm{C}$ & 5.17229500 & 3.62473200 & 0.62140900 \\
\hline $\mathrm{C}$ & -7.89409900 & 7.01418300 & -1.41510400 \\
\hline $\mathrm{C}$ & -8.90634500 & 7.20556900 & -2.56027400 \\
\hline $\mathrm{C}$ & -6.55595900 & 7.67114800 & -1.83395600 \\
\hline $\mathrm{C}$ & -8.44352500 & 7.72824100 & -0.15626600 \\
\hline $\mathrm{C}$ & -5.15367900 & -7.10873700 & 1.05869600 \\
\hline
\end{tabular}




\begin{tabular}{|c|c|c|c|}
\hline $\mathrm{C}$ & -4.21429700 & -7.75939900 & 0.02589400 \\
\hline $\mathrm{C}$ & -6.48064600 & -7.90567900 & 1.07714500 \\
\hline $\mathrm{C}$ & -4.48354400 & -7.19911400 & 2.45138900 \\
\hline $\mathrm{C}$ & 5.14553100 & 7.10234600 & -1.07948100 \\
\hline $\mathrm{C}$ & 4.20641000 & 7.75545700 & -0.04798500 \\
\hline $\mathrm{C}$ & 4.47486100 & 7.18908000 & -2.47214900 \\
\hline $\mathrm{C}$ & 6.47234800 & 7.89947600 & -1.10043900 \\
\hline $\mathrm{C}$ & 7.94152200 & -6.99373200 & 1.46797200 \\
\hline $\mathrm{C}$ & 9.45727200 & -7.26530300 & 1.30859200 \\
\hline $\mathrm{C}$ & 7.17522300 & -7.99515000 & 0.58340400 \\
\hline $\mathrm{C}$ & 7.53065700 & -7.23163800 & 2.94195900 \\
\hline $\mathrm{C}$ & -0.90864600 & -2.61356100 & 0.84469000 \\
\hline $\mathrm{C}$ & 0.07781400 & -3.53861700 & 1.16588900 \\
\hline $\mathrm{C}$ & 1.45409300 & -3.23242600 & 1.08393500 \\
\hline $\mathrm{C}$ & 1.82866900 & -1.96405100 & 0.67113300 \\
\hline $\mathrm{H}$ & -12.75772900 & 1.81691100 & 1.83175200 \\
\hline $\mathrm{H}$ & -8.58980700 & 2.68486600 & 1.42913600 \\
\hline $\mathrm{H}$ & -11.94869100 & -4.03784700 & -1.27044300 \\
\hline $\mathrm{H}$ & -7.72323300 & -3.37937200 & -1.49258300 \\
\hline $\mathrm{H}$ & -13.70609400 & -0.24339600 & 0.95380600 \\
\hline $\mathrm{H}$ & -13.40832000 & -2.40587100 & -0.19379500 \\
\hline $\mathrm{H}$ & -3.73972900 & -1.95842100 & 0.45190300 \\
\hline $\mathrm{H}$ & -4.60939800 & 2.76191600 & -0.91090800 \\
\hline $\mathrm{H}$ & 0.21187700 & 4.52829900 & -1.49412800 \\
\hline $\mathrm{H}$ & -2.19806000 & 3.98431500 & -1.34703200 \\
\hline $\mathrm{H}$ & 1.94062800 & 2.90602300 & -0.93066700 \\
\hline $\mathrm{H}$ & 4.60214400 & -2.76360300 & 0.91184400 \\
\hline $\mathrm{H}$ & 3.73261400 & 1.95412000 & -0.45977900 \\
\hline $\mathrm{H}$ & 11.94005800 & 4.04112900 & 1.24990700 \\
\hline $\mathrm{H}$ & 7.71589800 & 3.37786600 & 1.48248800 \\
\hline $\mathrm{H}$ & 8.58527500 & -2.69489200 & -1.42197800 \\
\hline $\mathrm{H}$ & 12.75001800 & -1.81943300 & -1.84033500 \\
\hline $\mathrm{H}$ & 13.69712900 & 0.24435400 & -0.97061500 \\
\hline $\mathrm{H}$ & 13.39929400 & 2.40922600 & 0.17254900 \\
\hline $\mathrm{H}$ & -11.41167900 & 4.88290500 & 0.75343400 \\
\hline $\mathrm{H}$ & -12.82586100 & 4.14823600 & 1.52625600 \\
\hline $\mathrm{H}$ & -12.04905900 & 5.55110700 & 2.26942200 \\
\hline $\mathrm{H}$ & -9.11229600 & 4.78774000 & 1.88650700 \\
\hline $\mathrm{H}$ & -8.89862100 & 3.87134800 & 3.39496500 \\
\hline $\mathrm{H}$ & -9.82760700 & 5.37313100 & 3.39093000 \\
\hline $\mathrm{H}$ & -12.53953900 & 2.87730200 & 3.78615600 \\
\hline $\mathrm{H}$ & -10.92663300 & 2.72309700 & 4.50123900 \\
\hline $\mathrm{H}$ & -11.74173600 & 4.29691300 & 4.46630000 \\
\hline $\mathrm{H}$ & -7.54131700 & -5.54201500 & -1.91993500 \\
\hline
\end{tabular}




\begin{tabular}{|c|c|c|c|}
\hline $\mathrm{H}$ & -8.20737500 & -6.37222200 & -3.32968100 \\
\hline $\mathrm{H}$ & -7.86871000 & -4.64147900 & -3.41704700 \\
\hline $\mathrm{H}$ & -10.30144100 & -4.29277500 & -4.20279900 \\
\hline $\mathrm{H}$ & -10.50162300 & -6.05186100 & -4.10781200 \\
\hline $\mathrm{H}$ & -11.64410700 & -4.98847800 & -3.28180600 \\
\hline $\mathrm{H}$ & -11.15875900 & -6.23824700 & -1.04835400 \\
\hline $\mathrm{H}$ & -10.04701600 & -7.29319500 & -1.92974700 \\
\hline $\mathrm{H}$ & -9.48706700 & -6.41636600 & -0.49168900 \\
\hline $\mathrm{H}$ & 9.47476400 & 6.41545200 & 0.47307900 \\
\hline $\mathrm{H}$ & 10.03650100 & 7.29508100 & 1.90873400 \\
\hline $\mathrm{H}$ & 11.14767300 & 6.23992100 & 1.02689500 \\
\hline $\mathrm{H}$ & 10.29821200 & 4.29846600 & 4.18594800 \\
\hline $\mathrm{H}$ & 11.63848200 & 4.99388000 & 3.26125800 \\
\hline $\mathrm{H}$ & 10.49664500 & 6.05757800 & 4.08778700 \\
\hline $\mathrm{H}$ & 7.86372700 & 4.64353600 & 3.40430000 \\
\hline $\mathrm{H}$ & 8.20047700 & 6.37447500 & 3.31354900 \\
\hline $\mathrm{H}$ & 7.53256200 & 5.54137000 & 1.90638800 \\
\hline $\mathrm{H}$ & 8.88205200 & -3.90951800 & -3.35844400 \\
\hline $\mathrm{H}$ & 9.82835600 & -5.40009800 & -3.35747800 \\
\hline $\mathrm{H}$ & 9.13028000 & -4.81008300 & -1.84594500 \\
\hline $\mathrm{H}$ & 12.84421600 & -4.13222400 & -1.55674100 \\
\hline $\mathrm{H}$ & 11.45286900 & -4.88154200 & -0.75675000 \\
\hline $\mathrm{H}$ & 12.07013600 & -5.54496200 & -2.28267700 \\
\hline $\mathrm{H}$ & 10.87403700 & -2.73862200 & -4.50135700 \\
\hline $\mathrm{H}$ & 11.71092800 & -4.30126000 & -4.47555300 \\
\hline $\mathrm{H}$ & 12.50085200 & -2.86826200 & -3.81351600 \\
\hline $\mathrm{H}$ & -5.81171700 & 3.60042100 & 1.05274600 \\
\hline $\mathrm{H}$ & -6.25373900 & 5.93871200 & 0.50797600 \\
\hline $\mathrm{H}$ & -8.97205900 & 4.70372400 & -2.58941000 \\
\hline $\mathrm{H}$ & -8.54236300 & 2.36971400 & -2.03646100 \\
\hline $\mathrm{H}$ & -4.74982200 & -3.15596800 & -1.51403500 \\
\hline $\mathrm{H}$ & -4.24358300 & -5.49844300 & -1.05572100 \\
\hline $\mathrm{H}$ & -6.74788600 & -5.35082500 & 2.43948900 \\
\hline $\mathrm{H}$ & -7.26709800 & -3.01286100 & 1.97013800 \\
\hline $\mathrm{H}$ & 8.53466200 & -2.36992000 & 2.04052900 \\
\hline $\mathrm{H}$ & 8.96417700 & -4.70676500 & 2.59126500 \\
\hline $\mathrm{H}$ & 6.24986200 & -5.94046800 & -0.51026900 \\
\hline $\mathrm{H}$ & 5.80741200 & -3.60323100 & -1.05063400 \\
\hline $\mathrm{H}$ & 7.25916100 & 3.00448300 & -1.98142400 \\
\hline $\mathrm{H}$ & 6.73945000 & 5.34117700 & -2.45645500 \\
\hline $\mathrm{H}$ & 4.23660800 & 5.49729900 & 1.03943100 \\
\hline $\mathrm{H}$ & 4.74329700 & 3.15603100 & 1.50340100 \\
\hline $\mathrm{H}$ & -8.55972300 & 6.75153000 & -3.49551500 \\
\hline $\mathrm{H}$ & -9.04768900 & 8.27468500 & -2.74886900 \\
\hline
\end{tabular}




\begin{tabular}{|c|c|c|c|}
\hline $\mathrm{H}$ & -9.88789400 & 6.78441800 & -2.31496400 \\
\hline $\mathrm{H}$ & -6.14436000 & 7.19150100 & -2.72932100 \\
\hline $\mathrm{H}$ & -6.71364600 & 8.73142900 & -2.06108000 \\
\hline $\mathrm{H}$ & -5.80300700 & 7.61119200 & -1.04064800 \\
\hline $\mathrm{H}$ & -9.39599900 & 7.28950500 & 0.16198300 \\
\hline $\mathrm{H}$ & -7.74743700 & 7.67168200 & 0.68723600 \\
\hline $\mathrm{H}$ & -8.61628700 & 8.78850400 & -0.37207600 \\
\hline $\mathrm{H}$ & -4.64431600 & -7.75209900 & -0.98204700 \\
\hline $\mathrm{H}$ & -3.23814300 & -7.26162500 & -0.01250700 \\
\hline $\mathrm{H}$ & -4.03753600 & -8.80494200 & 0.29847500 \\
\hline $\mathrm{H}$ & -6.97823900 & -7.86580700 & 0.10159000 \\
\hline $\mathrm{H}$ & -7.17953700 & -7.52342600 & 1.82830100 \\
\hline $\mathrm{H}$ & -6.28455800 & -8.95756700 & 1.31348400 \\
\hline $\mathrm{H}$ & -3.53421500 & -6.65090100 & 2.46901700 \\
\hline $\mathrm{H}$ & -5.12150600 & -6.79109800 & 3.24211400 \\
\hline $\mathrm{H}$ & -4.27436600 & -8.24533700 & 2.70125400 \\
\hline $\mathrm{H}$ & 3.23037200 & 7.25757800 & -0.00795100 \\
\hline $\mathrm{H}$ & 4.02933000 & 8.80026300 & -0.32316900 \\
\hline $\mathrm{H}$ & 4.63680700 & 7.75081500 & 0.95981200 \\
\hline $\mathrm{H}$ & 3.52563300 & 6.64064100 & -2.48805000 \\
\hline $\mathrm{H}$ & 5.11260800 & 6.77919400 & -3.26207800 \\
\hline $\mathrm{H}$ & 4.26539000 & 8.23462900 & -2.72457800 \\
\hline $\mathrm{H}$ & 7.17102700 & 7.51543100 & -1.85087700 \\
\hline $\mathrm{H}$ & 6.27599000 & 8.95072500 & -1.33938400 \\
\hline $\mathrm{H}$ & 6.97030800 & 7.86217200 & -0.12496900 \\
\hline $\mathrm{H}$ & 10.06181500 & -6.61612100 & 1.95045700 \\
\hline $\mathrm{H}$ & 9.68327600 & -8.30212600 & 1.58151200 \\
\hline $\mathrm{H}$ & 9.77924400 & -7.11139300 & 0.27229900 \\
\hline $\mathrm{H}$ & 7.44160200 & -7.89585800 & -0.47509900 \\
\hline $\mathrm{H}$ & 7.42293600 & -9.01702100 & 0.88825100 \\
\hline $\mathrm{H}$ & 6.08928000 & -7.88008900 & 0.67874300 \\
\hline $\mathrm{H}$ & 6.45889500 & -7.05401500 & 3.08691000 \\
\hline $\mathrm{H}$ & 7.74341300 & -8.26775400 & 3.22801700 \\
\hline $\mathrm{H}$ & 8.07629600 & -6.57902000 & 3.63131100 \\
\hline $\mathrm{H}$ & -1.94802000 & -2.90841900 & 0.92851500 \\
\hline $\mathrm{H}$ & -0.21932200 & -4.53032400 & 1.49330400 \\
\hline $\mathrm{H}$ & 2.19062400 & -3.98630200 & 1.34635500 \\
\hline
\end{tabular}

\section{OT $9 b^{2++}$}

Energy (with Zero Point Energy correction) $=-4562.590503(-4560.652175)$ Hartree Free energy $(298 \mathrm{~K})=-4560.801612$ Hartree

Zero number of imaginary frequency

23

$\begin{array}{llll}\text { C } & 11.79891500 & -1.53219700 & 1.49293800\end{array}$ 


\begin{tabular}{|c|c|c|c|}
\hline $\mathrm{C}$ & 11.63434300 & -0.29307800 & 0.85970400 \\
\hline $\mathrm{C}$ & 10.33505300 & 0.10346600 & 0.43350000 \\
\hline $\mathrm{C}$ & 9.24699000 & -0.81751900 & 0.55045200 \\
\hline $\mathrm{C}$ & 9.47140900 & -2.03368900 & 1.24812000 \\
\hline $\mathrm{C}$ & 10.72088200 & -2.40192700 & 1.74174900 \\
\hline $\mathrm{C}$ & 10.14057600 & 1.39036400 & -0.13811100 \\
\hline $\mathrm{C}$ & 8.83227900 & 1.85672500 & -0.45024000 \\
\hline $\mathrm{C}$ & 7.68322000 & 0.98416700 & -0.14336300 \\
\hline $\mathrm{C}$ & 7.93782400 & -0.42312400 & 0.05484400 \\
\hline $\mathrm{C}$ & 11.27051700 & 2.23024300 & -0.39089100 \\
\hline $\mathrm{C}$ & 11.07975800 & 3.46085700 & -1.03422700 \\
\hline $\mathrm{C}$ & 9.81782600 & 3.88556800 & -1.45135700 \\
\hline $\mathrm{C}$ & 8.71187900 & 3.07420000 & -1.13106900 \\
\hline $\mathrm{C}$ & 12.74582900 & 0.58672800 & 0.63527200 \\
\hline $\mathrm{C}$ & 12.57169900 & 1.78824500 & 0.01726100 \\
\hline $\mathrm{C}$ & 6.35656800 & 1.44185000 & -0.01444400 \\
\hline $\mathrm{C}$ & 5.27254200 & 0.49649600 & -0.01755700 \\
\hline $\mathrm{C}$ & 5.54392800 & -0.89427800 & -0.27372400 \\
\hline $\mathrm{C}$ & 6.88381900 & -1.36320600 & -0.19144400 \\
\hline $\mathrm{C}$ & 3.92928200 & 0.91168200 & 0.16520800 \\
\hline $\mathrm{C}$ & 2.87832600 & 0.03005400 & -0.03562000 \\
\hline $\mathrm{C}$ & 3.15474800 & -1.31910500 & -0.45577100 \\
\hline $\mathrm{C}$ & 4.44838900 & -1.76310500 & -0.57358100 \\
\hline $\mathrm{C}$ & 0.12988200 & -3.58848400 & -1.14741400 \\
\hline $\mathrm{C}$ & 1.51123300 & -3.26593800 & -1.06243200 \\
\hline $\mathrm{C}$ & 1.87172700 & -1.99723700 & -0.66052300 \\
\hline $\mathrm{C}$ & 0.85738600 & -1.04920800 & -0.34228600 \\
\hline $\mathrm{C}$ & -0.53117800 & -1.35533500 & -0.41951200 \\
\hline $\mathrm{C}$ & -0.86307100 & -2.67709700 & -0.83926800 \\
\hline $\mathrm{C}$ & 1.42896200 & 0.19262800 & 0.04310800 \\
\hline $\mathrm{C}$ & 0.55688200 & 1.25671300 & 0.39161600 \\
\hline $\mathrm{C}$ & -0.82973900 & 0.94947600 & 0.31501400 \\
\hline $\mathrm{C}$ & -1.40067300 & -0.29223400 & -0.06912600 \\
\hline $\mathrm{C}$ & -2.85421700 & -0.12689100 & 0.01259200 \\
\hline $\mathrm{C}$ & -3.12541300 & 1.22441000 & 0.42871500 \\
\hline $\mathrm{C}$ & -4.42064300 & 1.68792700 & 0.53019700 \\
\hline $\mathrm{C}$ & -5.51727800 & 0.83210800 & 0.24668000 \\
\hline $\mathrm{C}$ & -5.25654800 & -0.56210700 & -0.00117200 \\
\hline $\mathrm{C}$ & -3.90965100 & -0.99230000 & -0.19776500 \\
\hline $\mathrm{C}$ & -6.85865300 & 1.33422900 & 0.14166500 \\
\hline $\mathrm{C}$ & -7.92502600 & 0.43807400 & -0.08281500 \\
\hline $\mathrm{C}$ & -7.68404600 & -0.96983600 & 0.12774300 \\
\hline $\mathrm{C}$ & -6.35012400 & -1.47585800 & -0.01244300 \\
\hline $\mathrm{C}$ & -9.27661800 & 0.85167900 & -0.50044100 \\
\hline
\end{tabular}




\begin{tabular}{|c|c|c|c|}
\hline $\mathrm{C}$ & -10.35881600 & -0.04848600 & -0.28394000 \\
\hline $\mathrm{C}$ & -10.13564100 & -1.32367100 & 0.30086800 \\
\hline $\mathrm{C}$ & -8.80649500 & -1.80304500 & 0.52665000 \\
\hline $\mathrm{C}$ & -11.24347200 & -2.15455200 & 0.63237900 \\
\hline $\mathrm{C}$ & -11.01404200 & -3.37430600 & 1.28220800 \\
\hline $\mathrm{C}$ & -9.72281400 & -3.81044300 & 1.63742400 \\
\hline $\mathrm{C}$ & -8.64641100 & -3.02453800 & 1.23475000 \\
\hline $\mathrm{C}$ & -9.53690700 & 2.03898800 & -1.19575300 \\
\hline $\mathrm{C}$ & -10.82704300 & 2.41071100 & -1.62245200 \\
\hline $\mathrm{C}$ & -11.88884200 & 1.56499000 & -1.29913700 \\
\hline $\mathrm{C}$ & -11.68738500 & 0.34144700 & -0.64551400 \\
\hline $\mathrm{C}$ & -12.77900100 & -0.53241200 & -0.33218100 \\
\hline $\mathrm{C}$ & -12.57043200 & -1.72201700 & 0.29907800 \\
\hline $\mathrm{C}$ & 7.18654100 & -2.78844700 & -0.46759900 \\
\hline $\mathrm{C}$ & 10.96290600 & -3.70386700 & 2.52465700 \\
\hline $\mathrm{C}$ & 11.90839800 & -4.61271100 & 1.70150000 \\
\hline $\mathrm{C}$ & 9.65873100 & -4.47528600 & 2.79737300 \\
\hline $\mathrm{C}$ & 11.62026400 & -3.37429500 & 3.88700100 \\
\hline $\mathrm{C}$ & 9.67458200 & 5.21096900 & -2.22173200 \\
\hline $\mathrm{C}$ & 8.23161600 & 5.46725600 & -2.69533600 \\
\hline $\mathrm{C}$ & 10.59009100 & 5.18484600 & -3.46977000 \\
\hline $\mathrm{C}$ & 10.09937800 & 6.37685700 & -1.29564800 \\
\hline $\mathrm{C}$ & 6.04360400 & 2.88079600 & 0.22308300 \\
\hline $\mathrm{C}$ & -6.10831700 & -2.91454500 & -0.27595300 \\
\hline $\mathrm{C}$ & -7.09159900 & 2.78904200 & 0.36791700 \\
\hline $\mathrm{C}$ & -9.55862100 & -5.11391300 & 2.43723900 \\
\hline $\mathrm{C}$ & -10.07829400 & -6.29578800 & 1.58241000 \\
\hline $\mathrm{C}$ & -10.38129800 & -5.02214600 & 3.74569900 \\
\hline $\mathrm{C}$ & -8.09141800 & -5.39122400 & 2.81223500 \\
\hline $\mathrm{C}$ & -11.09577300 & 3.70868100 & -2.40540800 \\
\hline $\mathrm{C}$ & -9.80111400 & 4.45972200 & -2.76843700 \\
\hline $\mathrm{C}$ & -11.97575200 & 4.64203800 & -1.53800300 \\
\hline $\mathrm{C}$ & -11.84046600 & 3.37405700 & -3.72071900 \\
\hline $\mathrm{C}$ & 6.54641000 & -3.84838000 & 0.20624300 \\
\hline $\mathrm{C}$ & 6.83079400 & -5.17098300 & -0.11235500 \\
\hline $\mathrm{C}$ & 7.74571800 & -5.51240700 & -1.12828900 \\
\hline $\mathrm{C}$ & 8.37702800 & -4.45068600 & -1.79547900 \\
\hline $\mathrm{C}$ & 8.11626200 & -3.12221600 & -1.46871700 \\
\hline $\mathrm{C}$ & 5.20121100 & 3.61802900 & -0.62227600 \\
\hline $\mathrm{C}$ & 4.90604700 & 4.95712500 & -0.35514400 \\
\hline $\mathrm{C}$ & 5.42394000 & 5.61439500 & 0.77051900 \\
\hline $\mathrm{C}$ & 6.26083300 & 4.86180300 & 1.61600100 \\
\hline $\mathrm{C}$ & 6.57009900 & 3.53286000 & 1.35130600 \\
\hline $\mathrm{C}$ & -7.91140200 & 3.20928100 & 1.42962000 \\
\hline
\end{tabular}




\begin{tabular}{|c|c|c|c|}
\hline $\mathrm{C}$ & -8.11034000 & 4.56088500 & 1.68383400 \\
\hline $\mathrm{C}$ & -7.51875500 & 5.56306000 & 0.89124000 \\
\hline $\mathrm{C}$ & -6.70768900 & 5.13418200 & -0.16970200 \\
\hline $\mathrm{C}$ & -6.48940200 & 3.77838800 & -0.42360500 \\
\hline $\mathrm{C}$ & -6.78023700 & -3.55284900 & -1.33849700 \\
\hline $\mathrm{C}$ & -6.53111200 & -4.88399900 & -1.64370700 \\
\hline $\mathrm{C}$ & -5.62353500 & -5.66177900 & -0.89868200 \\
\hline $\mathrm{C}$ & -4.97096000 & -5.02504100 & 0.16994100 \\
\hline $\mathrm{C}$ & -5.19593700 & -3.68199100 & 0.47045600 \\
\hline $\mathrm{C}$ & 8.01128700 & -6.98849800 & -1.46368600 \\
\hline $\mathrm{C}$ & 9.04387000 & -7.15562200 & -2.59438200 \\
\hline $\mathrm{C}$ & 6.68613600 & -7.65222400 & -1.91267500 \\
\hline $\mathrm{C}$ & 8.54675600 & -7.71347700 & -0.20484000 \\
\hline $\mathrm{C}$ & 5.11245100 & 7.08367300 & 1.10220100 \\
\hline $\mathrm{C}$ & 4.19656600 & 7.73930400 & 0.05155100 \\
\hline $\mathrm{C}$ & 6.43328400 & 7.88838600 & 1.16330400 \\
\hline $\mathrm{C}$ & 4.40536400 & 7.15660600 & 2.47738500 \\
\hline $\mathrm{C}$ & -5.38332000 & -7.13355300 & -1.26971900 \\
\hline $\mathrm{C}$ & -4.37663300 & -7.81896500 & -0.32663800 \\
\hline $\mathrm{C}$ & -4.82775700 & -7.20718500 & -2.71348700 \\
\hline $\mathrm{C}$ & -6.72219600 & -7.90760100 & -1.19668700 \\
\hline $\mathrm{C}$ & -7.76754700 & 7.04668400 & 1.21063800 \\
\hline $\mathrm{C}$ & -9.28636900 & 7.33879000 & 1.14888900 \\
\hline $\mathrm{C}$ & -7.05455100 & 7.98601300 & 0.21973900 \\
\hline $\mathrm{C}$ & -7.24455000 & 7.35420000 & 2.63506100 \\
\hline $\mathrm{C}$ & 0.88601700 & 2.58041000 & 0.80652000 \\
\hline $\mathrm{C}$ & -0.10747200 & 3.49508800 & 1.11043600 \\
\hline $\mathrm{C}$ & -1.48676100 & 3.17231400 & 1.02594400 \\
\hline $\mathrm{C}$ & -1.84475200 & 1.89924900 & 0.62904000 \\
\hline $\mathrm{H}$ & 12.79784300 & -1.80473500 & 1.82111500 \\
\hline $\mathrm{H}$ & 8.62999500 & -2.68201200 & 1.43208100 \\
\hline $\mathrm{H}$ & 11.95359400 & 4.07742900 & -1.22271900 \\
\hline $\mathrm{H}$ & 7.73108700 & 3.39761600 & -1.44222600 \\
\hline $\mathrm{H}$ & 13.73347400 & 0.26721800 & 0.95561600 \\
\hline $\mathrm{H}$ & 13.42076500 & 2.44014800 & -0.16840200 \\
\hline $\mathrm{H}$ & 3.74283700 & 1.93861700 & 0.44455800 \\
\hline $\mathrm{H}$ & 4.64930600 & -2.77999700 & -0.88696900 \\
\hline $\mathrm{H}$ & -0.15188700 & -4.58715600 & -1.46768100 \\
\hline $\mathrm{H}$ & 2.25347400 & -4.01748700 & -1.31613500 \\
\hline $\mathrm{H}$ & -1.90032500 & -2.98088300 & -0.92279000 \\
\hline $\mathrm{H}$ & -4.60980000 & 2.71736200 & 0.80786400 \\
\hline $\mathrm{H}$ & -3.73516400 & -2.01263000 & -0.50756800 \\
\hline $\mathrm{H}$ & -11.87706400 & -3.98176600 & 1.53902900 \\
\hline $\mathrm{H}$ & -7.64925600 & -3.33502200 & 1.50205800 \\
\hline
\end{tabular}




\begin{tabular}{|c|c|c|c|}
\hline $\mathrm{H}$ & -8.71099300 & 2.69115800 & -1.43200400 \\
\hline $\mathrm{H}$ & -12.90480000 & 1.83588000 & -1.57040100 \\
\hline $\mathrm{H}$ & -13.78451300 & -0.22022500 & -0.60002700 \\
\hline $\mathrm{H}$ & -13.40578700 & -2.37040700 & 0.54771500 \\
\hline $\mathrm{H}$ & 11.46152200 & -4.87188800 & 0.73485800 \\
\hline $\mathrm{H}$ & 12.87411900 & -4.13378000 & 1.50751700 \\
\hline $\mathrm{H}$ & 12.10495400 & -5.54245700 & 2.24724600 \\
\hline $\mathrm{H}$ & 9.16323200 & -4.78978700 & 1.87253900 \\
\hline $\mathrm{H}$ & 8.94936800 & -3.88139900 & 3.38587300 \\
\hline $\mathrm{H}$ & 9.88442800 & -5.37921400 & 3.37258900 \\
\hline $\mathrm{H}$ & 12.58668400 & -2.87274100 & 3.77306300 \\
\hline $\mathrm{H}$ & 10.97435200 & -2.72787400 & 4.49169000 \\
\hline $\mathrm{H}$ & 11.79595700 & -4.29804800 & 4.44922100 \\
\hline $\mathrm{H}$ & 7.53058300 & 5.55512800 & -1.85830300 \\
\hline $\mathrm{H}$ & 8.19509400 & 6.40782900 & -3.25506500 \\
\hline $\mathrm{H}$ & 7.87429800 & 4.67481400 & -3.36353100 \\
\hline $\mathrm{H}$ & 10.31569100 & 4.36577300 & -4.14404400 \\
\hline $\mathrm{H}$ & 10.49413900 & 6.12551300 & -4.02346300 \\
\hline $\mathrm{H}$ & 11.64592400 & 5.06482800 & -3.20740900 \\
\hline $\mathrm{H}$ & 11.13609000 & 6.27480300 & -0.95817600 \\
\hline $\mathrm{H}$ & 10.01675100 & 7.33057200 & -1.82943000 \\
\hline $\mathrm{H}$ & 9.45982600 & 6.42688200 & -0.40700200 \\
\hline $\mathrm{H}$ & -9.50457700 & -6.39271900 & 0.65367900 \\
\hline $\mathrm{H}$ & -9.98269300 & -7.23313900 & 2.14192300 \\
\hline $\mathrm{H}$ & -11.13390000 & -6.17894400 & 1.31504700 \\
\hline $\mathrm{H}$ & -10.03708700 & -4.19157000 & 4.37201800 \\
\hline $\mathrm{H}$ & -11.45023400 & -4.88046300 & 3.55628100 \\
\hline $\mathrm{H}$ & -10.27114700 & -5.94798900 & 4.32095500 \\
\hline $\mathrm{H}$ & -7.67003000 & -4.59072400 & 3.43169600 \\
\hline $\mathrm{H}$ & -8.03370900 & -6.31773600 & 3.39285500 \\
\hline $\mathrm{H}$ & -7.45593700 & -5.51606100 & 1.92865400 \\
\hline $\mathrm{H}$ & -9.13488700 & 3.84917200 & -3.38935900 \\
\hline $\mathrm{H}$ & -10.05183000 & 5.35732000 & -3.34339800 \\
\hline $\mathrm{H}$ & -9.24675000 & 4.78248400 & -1.88064000 \\
\hline $\mathrm{H}$ & -12.93481300 & 4.17964000 & -1.28203900 \\
\hline $\mathrm{H}$ & -11.46828200 & 4.90471300 & -0.60284100 \\
\hline $\mathrm{H}$ & -12.18936100 & 5.56920500 & -2.08194000 \\
\hline $\mathrm{H}$ & -11.24286300 & 2.71024800 & -4.35559900 \\
\hline $\mathrm{H}$ & -12.03624400 & 4.29383500 & -4.28293700 \\
\hline $\mathrm{H}$ & -12.80479600 & 2.88870200 & -3.54019100 \\
\hline $\mathrm{H}$ & 5.85061500 & -3.63082800 & 1.01185200 \\
\hline $\mathrm{H}$ & 6.33044500 & -5.95551500 & 0.44730100 \\
\hline $\mathrm{H}$ & 9.08560900 & -4.65272400 & -2.59015100 \\
\hline $\mathrm{H}$ & 8.62050000 & -2.33066800 & -2.01480600 \\
\hline
\end{tabular}




\begin{tabular}{|c|c|c|c|}
\hline $\mathrm{H}$ & 4.79147500 & 3.15155800 & -1.51456400 \\
\hline $\mathrm{H}$ & 4.26435400 & 5.48802500 & -1.04925000 \\
\hline $\mathrm{H}$ & 6.68129800 & 5.32081200 & 2.50574600 \\
\hline $\mathrm{H}$ & 7.22013700 & 2.98788300 & 2.02968300 \\
\hline $\mathrm{H}$ & -8.38599200 & 2.46798900 & 2.06608300 \\
\hline $\mathrm{H}$ & -8.73906400 & 4.83978000 & 2.52401100 \\
\hline $\mathrm{H}$ & -6.23259500 & 5.85991500 & -0.81992100 \\
\hline $\mathrm{H}$ & -5.86386900 & 3.48935600 & -1.26432100 \\
\hline $\mathrm{H}$ & -7.48331600 & -2.98733600 & -1.94243900 \\
\hline $\mathrm{H}$ & -7.05607400 & -5.32648700 & -2.48465400 \\
\hline $\mathrm{H}$ & -4.27795400 & -5.57994300 & 0.79191900 \\
\hline $\mathrm{H}$ & -4.68889100 & -3.23624800 & 1.32175600 \\
\hline $\mathrm{H}$ & 8.70822300 & -6.69290200 & -3.52933800 \\
\hline $\mathrm{H}$ & 9.19824300 & -8.22087600 & -2.79417000 \\
\hline $\mathrm{H}$ & 10.01733400 & -6.72873700 & -2.32765600 \\
\hline $\mathrm{H}$ & 6.28455400 & -7.16492700 & -2.80836600 \\
\hline $\mathrm{H}$ & 6.85810600 & -8.70786600 & -2.15065800 \\
\hline $\mathrm{H}$ & 5.91986500 & -7.60971800 & -1.13117000 \\
\hline $\mathrm{H}$ & 9.48978100 & -7.27027800 & 0.13467600 \\
\hline $\mathrm{H}$ & 7.83643200 & -7.67466100 & 0.62768500 \\
\hline $\mathrm{H}$ & 8.73299600 & -8.76910000 & -0.43175600 \\
\hline $\mathrm{H}$ & 4.65261400 & 7.74368700 & -0.94491900 \\
\hline $\mathrm{H}$ & 3.22465800 & 7.23658500 & -0.01701900 \\
\hline $\mathrm{H}$ & 4.00689700 & 8.78130700 & 0.32922400 \\
\hline $\mathrm{H}$ & 6.95620100 & 7.86151300 & 0.20056800 \\
\hline $\mathrm{H}$ & 7.11489400 & 7.50210900 & 1.92819000 \\
\hline $\mathrm{H}$ & 6.22509800 & 8.93672100 & 1.40530400 \\
\hline $\mathrm{H}$ & 3.45967200 & 6.60205500 & 2.46456900 \\
\hline $\mathrm{H}$ & 5.02503200 & 6.74442100 & 3.28043800 \\
\hline $\mathrm{H}$ & 4.18272500 & 8.19894500 & 2.73214600 \\
\hline $\mathrm{H}$ & -3.39150600 & -7.33904300 & -0.35822900 \\
\hline $\mathrm{H}$ & -4.24156700 & -8.86178500 & -0.63117400 \\
\hline $\mathrm{H}$ & -4.72474800 & -7.82420500 & 0.71253700 \\
\hline $\mathrm{H}$ & -3.87467700 & -6.67250400 & -2.79814200 \\
\hline $\mathrm{H}$ & -5.52083200 & -6.77650800 & -3.44340300 \\
\hline $\mathrm{H}$ & -4.65593000 & -8.25184100 & -2.99583400 \\
\hline $\mathrm{H}$ & -7.47186300 & -7.49964900 & -1.88249700 \\
\hline $\mathrm{H}$ & -6.56336700 & -8.95717800 & -1.46804500 \\
\hline $\mathrm{H}$ & -7.13936200 & -7.88053300 & -0.18358700 \\
\hline $\mathrm{H}$ & -9.85118600 & 6.73522400 & 1.86702100 \\
\hline $\mathrm{H}$ & -9.47638600 & 8.39250600 & 1.38195000 \\
\hline $\mathrm{H}$ & -9.68689000 & 7.13695900 & 0.14881900 \\
\hline $\mathrm{H}$ & -7.39937600 & 7.83506000 & -0.80959200 \\
\hline $\mathrm{H}$ & -7.26464400 & 9.02704500 & 0.48582300 \\
\hline
\end{tabular}




$\begin{array}{lrrr}\mathrm{H} & -5.96628400 & 7.85647300 & 0.24161000 \\ \mathrm{H} & -6.16765000 & 7.16422500 & 2.70963600 \\ \mathrm{H} & -7.41977700 & 8.40761900 & 2.88086400 \\ \mathrm{H} & -7.74652900 & 6.74839300 & 3.39662400 \\ \mathrm{H} & 1.92253600 & 2.88670300 & 0.88833800 \\ \mathrm{H} & 0.17578400 & 4.49488500 & 1.42537200 \\ \mathrm{H} & -2.23087600 & 3.92358800 & 1.27463900\end{array}$

Mulliken spin densities:

$\begin{array}{rlc}1 & \mathrm{C} & 0.161009 \\ 2 & \mathrm{C} & -0.048310 \\ 3 & \mathrm{C} & 0.022037 \\ 4 & \mathrm{C} & 0.083323 \\ 5 & \mathrm{C} & 0.049165 \\ 6 & \mathrm{C} & -0.022903 \\ 7 & \mathrm{C} & 0.031142 \\ 8 & \mathrm{C} & 0.017351 \\ 9 & \mathrm{C} & -0.043948 \\ 10 & \mathrm{C} & 0.041505 \\ 11 & \mathrm{C} & 0.030432 \\ 12 & \mathrm{C} & -0.029414 \\ 13 & \mathrm{C} & 0.036882 \\ 14 & \mathrm{C} & -0.005351 \\ 15 & \mathrm{C} & 0.049363 \\ 16 & \mathrm{C} & -0.017486 \\ 17 & \mathrm{C} & 0.115901 \\ 18 & \mathrm{C} & -0.003031 \\ 19 & \mathrm{C} & -0.050685 \\ 20 & \mathrm{C} & 0.181405 \\ 21 & \mathrm{C} & 0.065759 \\ 22 & \mathrm{C} & 0.037161 \\ 23 & \mathrm{C} & -0.030967 \\ 24 & \mathrm{C} & 0.099067 \\ 25 & \mathrm{C} & -0.012170 \\ 26 & \mathrm{C} & 0.033196 \\ 27 & \mathrm{C} & -0.000199 \\ 28 & \mathrm{C} & 0.002222 \\ 29 & \mathrm{C} & -0.017120 \\ 30 & \mathrm{C} & 0.037224 \\ 31 & \mathrm{C} & 0.022909 \\ 32 & \mathrm{C} & 0.008513 \\ 33 & \mathrm{C} & -0.025296 \\ 34 & \mathrm{C} & 0.095405 \\ 35 & \mathrm{C} & -0.062609 \\ 36 & \mathrm{C} & 0.068141\end{array}$




\begin{tabular}{|c|c|c|}
\hline 37 & $\mathrm{C}$ & 0.031508 \\
\hline 38 & $\mathrm{C}$ & 0.024621 \\
\hline 39 & $\mathrm{C}$ & -0.075472 \\
\hline 40 & $\mathrm{C}$ & 0.155150 \\
\hline 41 & $\mathrm{C}$ & 0.096823 \\
\hline 42 & $\mathrm{C}$ & -0.017987 \\
\hline 43 & $\mathrm{C}$ & 0.018516 \\
\hline 44 & $\mathrm{C}$ & 0.221196 \\
\hline 45 & $\mathrm{C}$ & 0.009913 \\
\hline 46 & $\mathrm{C}$ & 0.040996 \\
\hline 47 & $\mathrm{C}$ & 0.016578 \\
\hline 48 & $\mathrm{C}$ & 0.098408 \\
\hline 49 & $\mathrm{C}$ & -0.049031 \\
\hline 50 & $\mathrm{C}$ & 0.168622 \\
\hline 51 & $\mathrm{C}$ & -0.019316 \\
\hline 52 & $\mathrm{C}$ & 0.045692 \\
\hline 53 & $\mathrm{C}$ & 0.005363 \\
\hline 54 & $\mathrm{C}$ & 0.034978 \\
\hline 55 & $\mathrm{C}$ & -0.021932 \\
\hline 56 & $\mathrm{C}$ & 0.029875 \\
\hline 57 & $\mathrm{C}$ & -0.016419 \\
\hline 58 & $\mathrm{C}$ & 0.053015 \\
\hline 59 & $\mathrm{C}$ & -0.000958 \\
\hline 60 & $\mathrm{C}$ & 0.000965 \\
\hline 61 & $\mathrm{C}$ & 0.000863 \\
\hline 62 & $\mathrm{C}$ & -0.000051 \\
\hline 63 & $\mathrm{C}$ & 0.000451 \\
\hline 64 & $\mathrm{C}$ & -0.002098 \\
\hline 65 & $\mathrm{C}$ & 0.000097 \\
\hline 66 & $\mathrm{C}$ & 0.001260 \\
\hline 67 & $\mathrm{C}$ & 0.001566 \\
\hline 68 & $\mathrm{C}$ & -0.008315 \\
\hline 69 & $\mathrm{C}$ & -0.006606 \\
\hline 70 & $\mathrm{C}$ & -0.005311 \\
\hline 71 & $\mathrm{C}$ & 0.000754 \\
\hline 72 & $\mathrm{C}$ & 0.001035 \\
\hline 73 & $\mathrm{C}$ & 0.000645 \\
\hline 74 & $\mathrm{C}$ & -0.000035 \\
\hline 75 & $\mathrm{C}$ & -0.002002 \\
\hline 76 & $\mathrm{C}$ & 0.000096 \\
\hline 77 & $\mathrm{C}$ & 0.001674 \\
\hline 78 & $\mathrm{C}$ & 0.001343 \\
\hline 79 & $\mathrm{C}$ & 0.022001 \\
\hline 80 & $\mathrm{C}$ & -0.009312 \\
\hline
\end{tabular}




\begin{tabular}{|c|c|c|}
\hline 81 & $\mathrm{C}$ & 0.043053 \\
\hline 82 & $\mathrm{C}$ & -0.017222 \\
\hline 83 & $\mathrm{C}$ & 0.033421 \\
\hline 84 & $\mathrm{C}$ & 0.009208 \\
\hline 85 & $\mathrm{C}$ & -0.003660 \\
\hline 86 & $\mathrm{C}$ & 0.009121 \\
\hline 87 & $\mathrm{C}$ & -0.001953 \\
\hline 88 & $\mathrm{C}$ & 0.006191 \\
\hline 89 & $\mathrm{C}$ & 0.005463 \\
\hline 90 & $\mathrm{C}$ & -0.001477 \\
\hline 91 & $\mathrm{C}$ & 0.010062 \\
\hline 92 & $\mathrm{C}$ & -0.003565 \\
\hline 93 & $\mathrm{C}$ & 0.008893 \\
\hline 94 & $\mathrm{C}$ & 0.032723 \\
\hline 95 & $\mathrm{C}$ & -0.018184 \\
\hline 96 & $\mathrm{C}$ & 0.045491 \\
\hline 97 & $\mathrm{C}$ & -0.011958 \\
\hline 98 & $\mathrm{C}$ & 0.029343 \\
\hline 99 & $\mathrm{C}$ & -0.002494 \\
\hline 100 & $\mathrm{C}$ & 0.000050 \\
\hline 101 & $\mathrm{C}$ & 0.001700 \\
\hline 102 & $\mathrm{C}$ & 0.001625 \\
\hline 103 & $\mathrm{C}$ & -0.000538 \\
\hline 104 & $\mathrm{C}$ & 0.000012 \\
\hline 105 & $\mathrm{C}$ & 0.000331 \\
\hline 106 & $\mathrm{C}$ & 0.000351 \\
\hline 107 & $\mathrm{C}$ & -0.002671 \\
\hline 108 & $\mathrm{C}$ & 0.000096 \\
\hline 109 & $\mathrm{C}$ & 0.001817 \\
\hline 110 & $\mathrm{C}$ & 0.001588 \\
\hline 111 & $\mathrm{C}$ & -0.000566 \\
\hline 112 & $\mathrm{C}$ & 0.000398 \\
\hline 113 & $\mathrm{C}$ & 0.000011 \\
\hline 114 & $\mathrm{C}$ & 0.000425 \\
\hline 115 & $\mathrm{C}$ & 0.053534 \\
\hline 116 & $\mathrm{C}$ & -0.005953 \\
\hline 117 & $\mathrm{C}$ & 0.047273 \\
\hline 118 & $\mathrm{C}$ & -0.002451 \\
\hline 119 & $\mathrm{H}$ & -0.006814 \\
\hline 120 & $\mathrm{H}$ & -0.002723 \\
\hline 121 & $\mathrm{H}$ & 0.001074 \\
\hline 122 & $\mathrm{H}$ & 0.000063 \\
\hline 123 & $\mathrm{H}$ & -0.002023 \\
\hline 124 & $\mathrm{H}$ & 0.000588 \\
\hline
\end{tabular}




$\begin{array}{ccc}125 & \mathrm{H} & -0.002623 \\ 126 & \mathrm{H} & -0.003612 \\ 127 & \mathrm{H} & 0.000336 \\ 128 & \mathrm{H} & -0.001536 \\ 129 & \mathrm{H} & -0.001578 \\ 130 & \mathrm{H} & -0.001326 \\ 131 & \mathrm{H} & -0.005802 \\ 132 & \mathrm{H} & -0.007115 \\ 133 & \mathrm{H} & -0.002667 \\ 134 & \mathrm{H} & -0.000409 \\ 135 & \mathrm{H} & 0.000721 \\ 136 & \mathrm{H} & 0.000525 \\ 137 & \mathrm{H} & -0.002169 \\ 138 & \mathrm{H} & -0.000108 \\ 139 & \mathrm{H} & 0.000025 \\ 140 & \mathrm{H} & 0.000782 \\ 141 & \mathrm{H} & -0.000056 \\ 142 & \mathrm{H} & 0.000060 \\ 143 & \mathrm{H} & -0.000030 \\ 144 & \mathrm{H} & 0.000047 \\ 145 & \mathrm{H} & -0.000024 \\ 146 & \mathrm{H} & 0.000663 \\ 147 & \mathrm{H} & -0.000013 \\ 148 & \mathrm{H} & -0.000043 \\ 149 & \mathrm{H} & 0.000042 \\ 150 & \mathrm{H} & 0.000006 \\ 151 & \mathrm{H} & 0.000263 \\ 152 & \mathrm{H} & -0.000063 \\ 153 & \mathrm{H} & -0.000071 \\ 154 & \mathrm{H} & 0.000391 \\ 155 & \mathrm{H} & -0.000028 \\ 156 & \mathrm{H} & -0.000100 \\ 157 & \mathrm{H} & 0.000904 \\ 158 & \mathrm{H} & 0.000022 \\ 159 & \mathrm{H} & -0.000026 \\ 160 & \mathrm{H} & 0.000040 \\ 161 & \mathrm{H} & 0.000761 \\ 162 & \mathrm{H} & 0.000068 \\ 163 & \mathrm{H} & -0.000034 \\ 168 & \mathrm{H} & -0.000065 \\ & \mathrm{H} & -0.000000045 \\ 165 & -0.000021 \\ 167\end{array}$




\begin{tabular}{|c|c|c|}
\hline 169 & $\mathrm{H}$ & -0.000038 \\
\hline 170 & $\mathrm{H}$ & 0.000415 \\
\hline 171 & $\mathrm{H}$ & 0.000006 \\
\hline 172 & $\mathrm{H}$ & 0.000273 \\
\hline 173 & $\mathrm{H}$ & -0.000064 \\
\hline 174 & $\mathrm{H}$ & -0.001136 \\
\hline 175 & $\mathrm{H}$ & 0.000653 \\
\hline 176 & $\mathrm{H}$ & 0.000805 \\
\hline 177 & $\mathrm{H}$ & -0.001493 \\
\hline 178 & $\mathrm{H}$ & -0.000486 \\
\hline 179 & $\mathrm{H}$ & 0.000377 \\
\hline 180 & $\mathrm{H}$ & 0.000240 \\
\hline 181 & $\mathrm{H}$ & -0.000209 \\
\hline 182 & $\mathrm{H}$ & -0.000183 \\
\hline 183 & $\mathrm{H}$ & 0.000208 \\
\hline 184 & $\mathrm{H}$ & 0.000367 \\
\hline 185 & $\mathrm{H}$ & -0.000458 \\
\hline 186 & $\mathrm{H}$ & -0.001450 \\
\hline 187 & $\mathrm{H}$ & 0.000922 \\
\hline 188 & $\mathrm{H}$ & 0.000764 \\
\hline 189 & $\mathrm{H}$ & -0.001491 \\
\hline 190 & $\mathrm{H}$ & 0.000039 \\
\hline 191 & $\mathrm{H}$ & -0.000047 \\
\hline 192 & $\mathrm{H}$ & 0.000027 \\
\hline 193 & $\mathrm{H}$ & -0.000011 \\
\hline 194 & $\mathrm{H}$ & 0.000655 \\
\hline 195 & $\mathrm{H}$ & -0.000057 \\
\hline 196 & $\mathrm{H}$ & -0.000038 \\
\hline 197 & $\mathrm{H}$ & -0.000072 \\
\hline 198 & $\mathrm{H}$ & 0.000419 \\
\hline 199 & $\mathrm{H}$ & 0.000009 \\
\hline 200 & $\mathrm{H}$ & 0.000007 \\
\hline 201 & $\mathrm{H}$ & -0.000004 \\
\hline 202 & $\mathrm{H}$ & -0.000001 \\
\hline 203 & $\mathrm{H}$ & -0.000012 \\
\hline 204 & $\mathrm{H}$ & 0.000122 \\
\hline 205 & $\mathrm{H}$ & -0.000004 \\
\hline 206 & $\mathrm{H}$ & -0.000016 \\
\hline 207 & $\mathrm{H}$ & 0.000099 \\
\hline 208 & $\mathrm{H}$ & 0.000029 \\
\hline 209 & $\mathrm{H}$ & -0.000039 \\
\hline 210 & $\mathrm{H}$ & 0.000043 \\
\hline 211 & $\mathrm{H}$ & -0.000019 \\
\hline 212 & $\mathrm{H}$ & -0.000080 \\
\hline
\end{tabular}




$\begin{array}{llr}213 & \mathrm{H} & 0.000484 \\ 214 & \mathrm{H} & -0.000066 \\ 215 & \mathrm{H} & 0.000465 \\ 216 & \mathrm{H} & -0.000023 \\ 217 & \mathrm{H} & -0.000015 \\ 218 & \mathrm{H} & 0.000142 \\ 219 & \mathrm{H} & -0.000000 \\ 220 & \mathrm{H} & 0.000013 \\ 221 & \mathrm{H} & -0.000005 \\ 222 & \mathrm{H} & 0.000005 \\ 223 & \mathrm{H} & -0.000004 \\ 224 & \mathrm{H} & 0.000118 \\ 225 & \mathrm{H} & -0.000019 \\ 226 & \mathrm{H} & -0.002374 \\ 227 & \mathrm{H} & -0.000112 \\ 228 & \mathrm{H} & -0.002181\end{array}$

